\title{
$F F_{0} \quad 21965$
}

\section{Research in Radiobiology}

\author{
Annual Report of Work in Progress \\ on the Chronic Toxicity Program
}

RADIOBIOLOGY DIVISION OF THE DEPARTMENT OF ANATOMY

UNIVERSITY OF UTAH COLLEGE OF MEDICINE

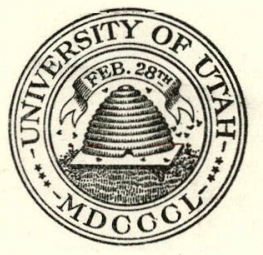

Respectfully Submitted by

THOMAS F. DOUGHERTY, Direvlong GEARANCE OBTAINED, RELLASSE TO

TRE PUOHC IS APPROVED. PROCEDURES

SEPTEMBER 30, 1964 


\section{DISCLAIMER}

This report was prepared as an account of work sponsored by an agency of the United States Government. Neither the United States Government nor any agency Thereof, nor any of their employees, makes any warranty, express or implied, or assumes any legal liability or responsibility for the accuracy, completeness, or usefulness of any information, apparatus, product, or process disclosed, or represents that its use would not infringe privately owned rights. Reference herein to any specific commercial product, process, or service by trade name, trademark, manufacturer, or otherwise does not necessarily constitute or imply its endorsement, recommendation, or favoring by the United States Government or any agency thereof. The views and opinions of authors expressed herein do not necessarily state or reflect those of the United States Government or any agency thereof. 


\section{DISCLAIMER}

Portions of this document may be illegible in electronic image products. Images are produced from the best available original document. 


\section{RESEARCH IN RADIOBIOLOGY}

Annual Report of Work in Progress

on the Chronic Toxicity Program

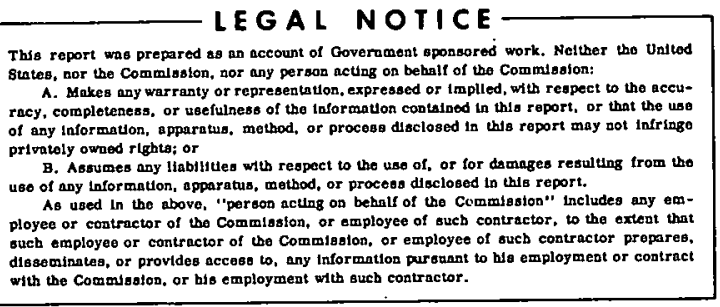

Radiobiology Division of the Department of Anatomy, University of Utah College of Medicine Respectfully Submitted by:

Thomas F. Dougherty, Director

September 30, 1964

Contract No. AT (ll-1)-119 
TABLE OF CONTENTS

Page

Availability of Previous Reports

Group Leaders and Sections

Current Census of the Beagle Colony

Preface to Injection Tables

Injection Tables

I. Toxicity Animals

A. Radium-226

B. Plutonium-239

C. Radium-228 (Mesothorium)

22

D. Thorium-228 (Radiothorium)

28

E. Strontium-90

II. Test Animals

A. Radium-226

40

B. Plutonium-239

46

C. Radium-228 (Mesothorium)

50

D. Thorium-228 (Radiothorium)

52

E. Strontium-90

F. Radium-224 (Quickradium)

G. Ancillary

Repeated Use of Anesthesia in an Experimental Dog Colony

Low Natural Incidence of Osteosarcomas in Beagles

The Effects of Ionizing Radiations on Endocrine Cells

IV. Increased Production of $17 \alpha, 20 \alpha$-Dihydroxyprogesterone

by Rat Testes After Irradiation 
Metabolism of the $\operatorname{Th}^{228}$ Decay Series in Adult Beagle Dogs

II. $\mathrm{Ra}^{224}(\mathrm{ThX}), \mathrm{Pb}^{212}$ (ThB), and $\mathrm{Bi}^{212}$ (ThC) 


\section{AVA ILABILITY OF PREVIOUS REPORTS}

Copies of our reports may be obtained from the office of Technical Services, U. S. Department of Commerce, Washington 25, D. C.

\begin{tabular}{|c|c|c|c|c|}
\hline Report & Date & Title & Photocopy & Microfilm \\
\hline$c 00-119-231$ & Sep 1964 & Research in Radiobiology & $\$ 17.20$ & $\$ 8.60$ \\
\hline$c 00-119-230$ & Jul 1964 & Safety Manual & 3.10 & 1.55 \\
\hline COO-119-229 & Mar 1964 & Research in Radiobiology & 13.00 & 5.84 \\
\hline $\mathrm{co0}-228$ & Sep 1963 & Research in Radiobiology & 13.00 & 5.84 \\
\hline $\mathrm{COO}-227$ & Mar 1963 & Research in Radiobiology & 15.50 & 7.22 \\
\hline $\mathrm{c} 00-226$ & Sep 1962 & Research in Radiobiology & 10.50 & 4.31 \\
\hline $\mathrm{C} 00-225$ & Mar 1962 & Research in Radiobiology & 10.50 & 4.28 \\
\hline $\mathrm{co0}-224$ & Sep 1961 & Research in Radiobiology & $9.10^{\circ}$ & 3.32 \\
\hline $\mathrm{co0}-223$ & Mar 1961 & Research in Radiobiology & 6.30 & 3.00 \\
\hline $\mathrm{C} 00-222$ & Sep 1960 & Research in Radiobiology & 22.80 & 7.20 \\
\hline $\mathrm{co0}-221$ & Aug 1960 & Interim Report of $\mathrm{Sr}^{90}$ & 3.30 & 2.40 \\
\hline $\mathrm{COO}-220$ & Mar 1960 & Research in Radiobiology & 34.80 & 9.90 \\
\hline $\operatorname{coo}-219$ & Sep 1959 & Semi-Annual Report & 10.80 & 3.90 \\
\hline $\operatorname{co0}-218$ & Mar 1959 & Annual Report & 34.80 & 9.90 \\
\hline AECU- 4112 & Feb 1959 & Radioactive Fallout: & 3.30 & 2.40 \\
\hline $\mathrm{C} 00-217$ & Sep 1958 & Semi-Annual Report & 28.80 & 8.40 \\
\hline $\mathrm{COO}-216$ & Mar 1958 & Escape of Radon \& Thoron & 9.30 & 6.30 \\
\hline $\mathrm{C} 00-215$ & Mar 1958 & Annual Report & 28.80 & 8.40 \\
\hline AECU-3583 & Sep 1957 & Semi-Annual Report & 19.80 & 6.30 \\
\hline AECU-3522 & Mar 1957 & Annual Report & 27.30 & 8.10 \\
\hline TID-16459 & Sep 1956 & Semi-Annual Report & 5.60 & 2.05 \\
\hline TID- 16458 & $\operatorname{Mar} 1956$ & Annua l - Rep ort & $10 \cdot 50$ & 4.40 \\
\hline AECU-3 109 & Sep 1955 & Semi-Annual Report & 12.30 & 4.50 \\
\hline AECU-3418 & Mar 1955 & Annual Report & 9.30 & 3.60 \\
\hline TID-7639 & \multicolumn{2}{|c|}{ Consultants Meeting } & 19.75 & 9.40 \\
\hline
\end{tabular}

Many of the above reports are also available from this laboratory. 
Division of Radiobiology, Department of Anatomy, University of Utah College of Medicine

\begin{tabular}{ll} 
Sirector & Group Leader \\
$\begin{array}{l}\text { Biochemistry } \\
\text { Bone }\end{array}$ & Prof. Thomas F. Dougherty \\
Chemistry & Dr. David L. Berliner \\
Clinic \& Pathology & Dr. Betsy J. Stover \\
Hematology & Dr. Glenn N. Taylor \\
Physics & Dr. Jean H. Dougherty \\
Radiology & Dr. Charles W. Mays \\
\hline
\end{tabular}




\section{CURRENT CENSUS OF THE BEAGLE COLONY}

September 30, 1964

\begin{tabular}{|c|c|c|c|}
\hline Assignment & . & Number 'of & Dogs \\
\hline Plutonium, toxicity & & 36 & \\
\hline Plutonium, test & & 9 & \\
\hline Radium, toxicity $\left(\mathrm{Ra}^{226}\right)$ & & 36 & \\
\hline Radium, test $\left(\mathrm{Ra}^{226}\right)$ & & . & \\
\hline Quickradium, test $\left(\mathrm{Ra}^{224}\right)$ & & 10 & \\
\hline Radiothorium, toxicity & & 54 & \\
\hline Radiothorium, test & . & 0 & \\
\hline Mesothorium, toxicity & & 50 & \\
\hline Mesothorium, test & & 0 & \\
\hline Strontium, toxicitty & & 70 & \\
\hline Strontium, test & & 1 & \\
\hline Controls, aging & & 44 & \\
\hline Controls, $x$-ray & & 5 & \\
\hline Unassigned (Male 31, Female 39) & & 70 & \\
\hline- & Total & 392 & \\
\hline
\end{tabular}




\section{INJECTION TABLES}

Tables I and II list the toxicity and test animals, respectively. Toxicity animals are those animals which will be maintained until sacrifice becomes a clinical necessity; test animals may be sacrificed as needed for special studies.

Dogs are put into the toxicity study groups of six or seven of the same sex (five or six dose levels plus one control). Litter mates are used whenever possible. Each animal receives the designated dose of one radionuclide in a single I.V. injection. 'l'he animals are injectcd at, approximately 17 months of age. At this age the skeleton is maturie with al1. epiphyses fused except those of the ribs. Twellve such groups have been injected for each of the five radionuclides, $\mathrm{Ra}^{226}, \mathrm{Pu}^{239}, \mathrm{Ra}^{228}$, $\mathrm{Th}^{228}$, and $\mathrm{Sr}^{90}$. The current injection program is limited to $\mathrm{Ra}^{226}$ and $\mathrm{Pu}^{239}$ at lower dose levels.

The five dose levels designated by integers are those specified at the early meetings of the consultants, and those designated by nonintegers have been added by the laboratory staff. Since those dose levels were originally specified"as "retained" doses, the actual injected doses are four times the desired "retained" doses of $\mathrm{Ra}^{226}, \mathrm{Ra}^{228}$ (Mesothorium), and $\mathrm{Sr}^{90}$, and 1.11 times the desired "retained" doses of $\mathrm{Pu}$ "239 and $\mathrm{Th}^{228}$ (Radiothorium).* The desired "retained" activities are the same for all

* Since radioactive decay and excretion occur continously, the term "retained" dose is obviously meaningless unless the time after injection is specified. Our present measurements indicate that

average $\mathrm{Ra}^{226}$ retention $=0.25$ after 2.71 days
àverage $\mathrm{Pu} 239$ retention $=0.90$ after 6 days
average $\mathrm{Ra} 228$ retention $=0.25$ after 2.14 days
average $\mathrm{Th} 228$ retention $=0.90$ after 6 . days
average $\mathrm{Sr} 90$ retention $=0.25$ after $135^{\circ}$. days


the radionuclides except $\mathrm{Sr}^{90}$, in which case they are greater by a factor of 10. Dose level 1 is the basis of the scheme, and is 10 times the maximum permissible $\mathrm{Ra}^{226}$ in man. Level $1=10 \times \frac{0.1 \mu \mathrm{c} \mathrm{Ra} 226}{70 \mathrm{~kg} \mathrm{man}}=$ 0.0143 "retained" $\mu \mathrm{c} / \mathrm{kg}$. All other dose levels are simple multiples of level $I$ as shown below.

$\begin{array}{lllrrr}\text { Level } & 0.1 & \text { is } 1 / 27 & \text { of level } & 1 \\ \text { Level } & 0.2 & \text { is } 1 / 9 & \text { of level } & 1 \\ \text { Level } 0.5 & \text { is } 1 / 3 & \text { of level } & 1 \\ \text { Level } 1.5 & \text { is } & 2 \text { times level } & 1 \\ \text { Level } 1.7 & \text { is } & 3 & \text { times level } & 1 \\ \text { Level } 2 & \text { is } & 6 & \text { times level } & 1 \\ \text { Level } 3 & \text { is } & 18 \text { times level } & 1 \\ \text { Level } 4 & \text { is } & 54 & \text { times level } & 1 \\ \text { Level } 5 & \text { is } & 162 \text { times level } & 1\end{array}$

The numbering system for the dogs has been built around the injection program and serves as a code to describe each dog's place in the experiment. The first letter tells the sex of toxicity animals $(M=$ male, $F=$ female). When the first letter is $T$, the dog is a test animal. M, F, or $\mathrm{T}$ is followed by a number which denotes chronological order of groups in the case of toxicity dogs and of individual test dogs.

Next comes: a: code letter for the radionuclide: $R=R^{226}, P=P u^{239}$ $\mathrm{M}=\mathrm{Ra}^{228}, \mathrm{~T}=\mathrm{Th}^{228}, \mathrm{~S}=\mathrm{Sr}^{90}, \mathrm{Q}=\mathrm{Ra}^{224}, \mathrm{~J}=\mathrm{Sr}{ }^{85}$, and $\mathrm{A}=$ Aging Control .

"A" following the regular dog number means that the dog is a replacement. "H" following the regular dog number means that the dog received its dose in more than one injection. "B", "C", or "D" denotes assignment to serial sacrifice schedule. Any of the above letters denoting a radionuclide may follow the final number, in which case the letter indicates that two rädionuclides were given. The dose level refers to the radionuclide appearing first in the identifying code.

- Example: MIR5 is a male animal in the first radium group at the highest dose level. 
Although MIR5, MIR4, MIR3, MIR2, MIRl, and MlRO constitute a group and were injected at the same time, the tables are arranged according to dose level to facilitate comparison of all the R5 animals, all the R4 animals, etc.

The average radiation doses to the skeleton in rads for $\mathrm{Pu}^{239}, \mathrm{Ra}^{228}$ and $\mathrm{Th}^{228}$ were calculated from equations and graphs in our Progress Report: C00-218, March 1959, pp. 121-145. $\mathrm{Ra}^{226}$ and $\mathrm{Sr}^{90}$ doses in this Report are completely revised: They are based on the individual retentions observed in each dog.

The coniditions listed in the injection tables under "Comments on Dead Dogs" present the lesions or factors that had the most prominent effect on the clinical status of the animal. For example; multiple rib fractures, which seldom produced symptoms, are not listed, even though their incidence was usually much higher than the crippling fractures involving the limb bones or mandible. The hematological changes have been omitted unless they were extreme. Increased rate of tooth loss, hepatic changes, eye lesions, and many other factors in the various syndromes have not been included because of space limitations. Over the years many soft tissue tumors have been removed surgically; these tumors were the subject of a separate report, Research in Radiobiology, September 30, $1963(\mathrm{cop}-228)$, pp. 95-108. In many instances, the conditions that have been listed were the reasons for sacrifice of the animal but they were not the immediate cause of death. Most of the animals were euthanized when death appeared eminent on when life could no longer be humanely prolonged. 


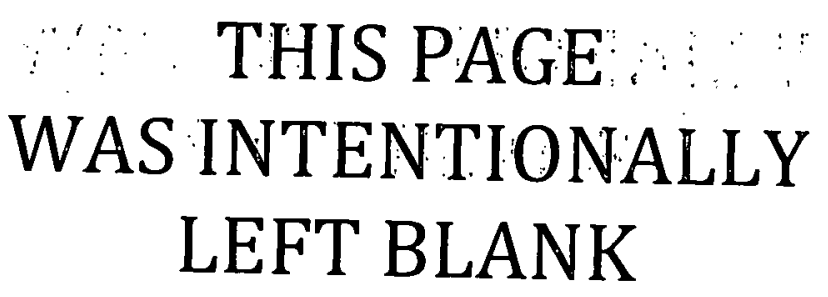


TABLE I.TOXICITY ANIMALS (SEPT.30.1964).

A. RADIUM-226*

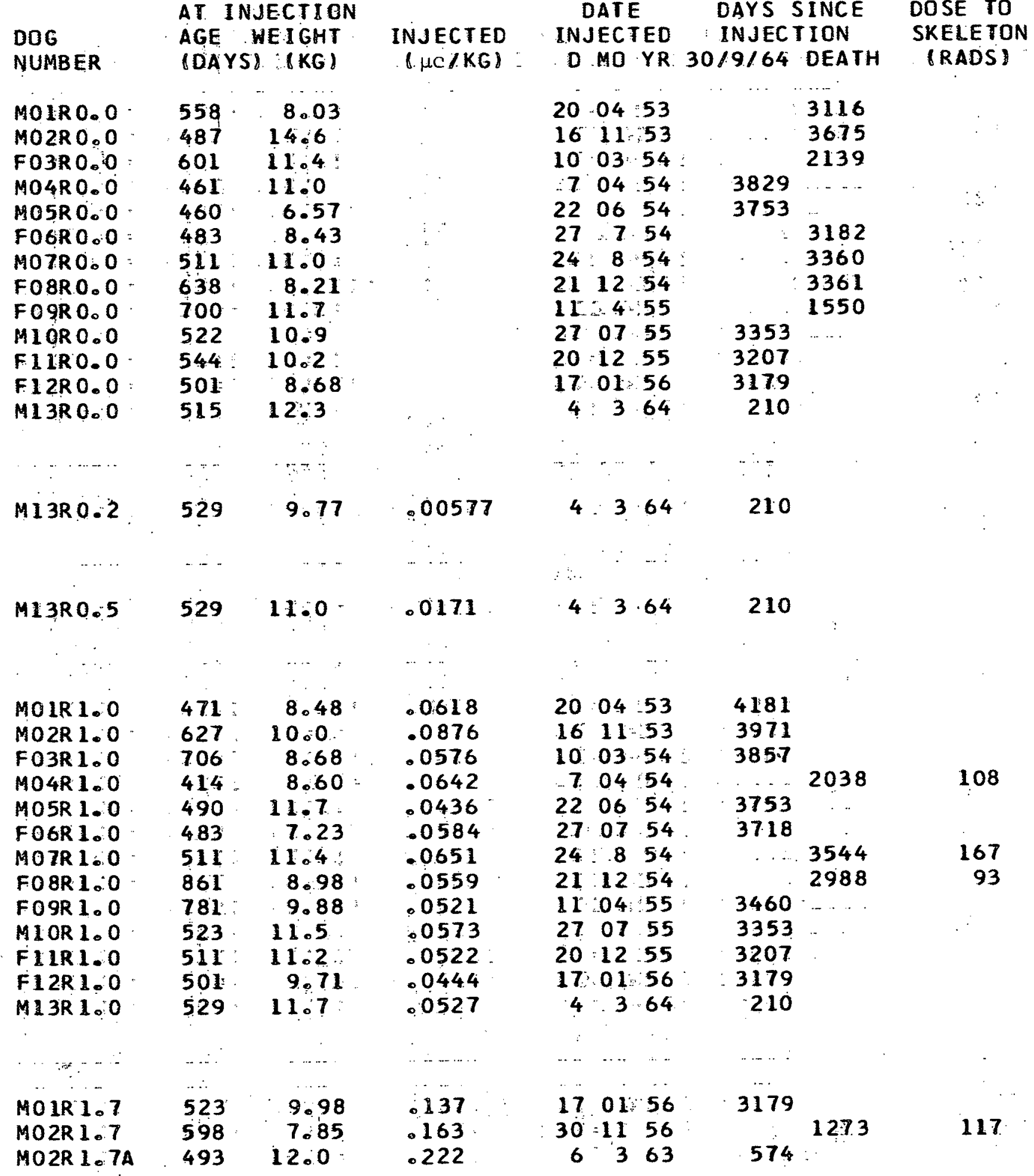


DOG

NUMB ER

MOLRO. 0

MO2R O.O

F03R 0.0

MO \&R O. 0

MOSR 0.0

FO6R 0.0

MOTR C.O

FO QR 0.0

FOSR 0.0

$M$ IOR 0.0

FIIRO.0

FI ZR O.O 0

MI 3 R 0.0

M $13 R 0.2$

$M 13 R 0.5$

MO1R 1.0

MO2R 1.0

FO3R 1.0

MO4R 1.0

MO5R 1.0

FO6R 1. 0

MOTR 1.0

FO QR 1.0

FO 9R 1:0

MIOR 1. 0

F1IR 1.0

F1 $2 R 1.0$

M1 131.0
COMMENTS ON DEAC DOGS

SEMINOMA I LYMPHOS ARCOMA

TRANSITIONAL: CELL CARCINOMA.

STATUS EPILEPTICUS

STATUS EPILEPTICUS

STATUS EPILEPTICUS, NEPHRITIS

PANCREATIC ADENOCARCINOMA

AORTIC BCOY. TUMOR

\section{TRAUMA}

STATUS EPILEPTICUS

LYMPHOSARCOMA

MOIR 1.7

MO2R 1.7

MO2R 1. TA

LYMPHOSARCOMA 


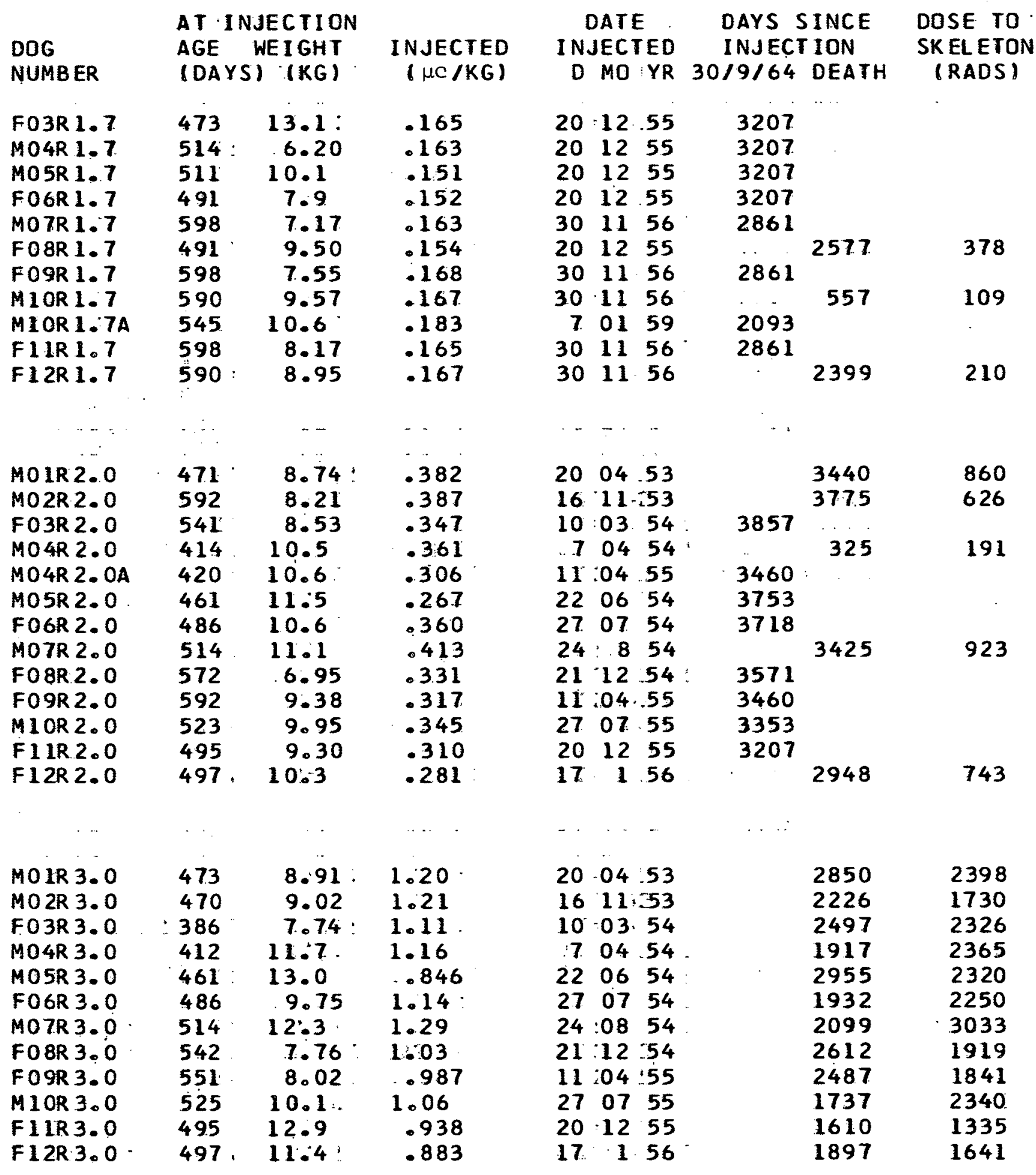


DOG

NUMBER

FO3R 1.7

MO4R 2.7

MO5R 1.7

FO6R 1.7

MOTR 1.7

F.08R 1.7

FO9R 1.7

MIOR 1.7

MIOR 1.7A

FIIR 1.7

FI2R 1.7

MOIR 2.0

MO2R 2.O

FO $3 R 2.0$

MO LR 2. 0

MO4R 2. OA

MOSR 2. 0

FOGR 2.0

MOTR2.0

FO \&R 2.0

FOSR 2.0

M IOR 2.0

F1LR2. 0

F12R2.0

MOLR 3.0

MO2R 3.0

F0 3R 3.0

MO4R 3.0

MOSR 3.0

F.OGR 3.0

MOTR 3.0

FOBR 3.0

FOSR 3.0

M IOR 3.0

F 1 IR 3.0

F1 $2 R 3.0$
COMMENTS ON CEAD DOES
DRUG ALLERGY

TRAUMA

NOT DETERMI NED

HEMANG I OSARCOMA

OSTEOSARCOMA

PERFORATED ILEUM

OSTEOSARCOMA CUSHING SYNDROME

MAMMARY ADENOCARCINOMA

DSTEOSARCOMA

OSTEOSARCDMA

DSTEOSARCOMA

OSTEOSARCOMA

OSTEOSARCOMA

OSTEOSARCOMA

DSTE OSARCOMA

OS TE OSARCOMA

OSTEOSARCOMA

OSTEOSARCOMA

PYOMETRITIS * SECONDARY PERITONITIS

OSTEOSARCOMA 


\begin{tabular}{|c|c|c|c|c|c|c|c|c|c|}
\hline $\begin{array}{l}\text { DOG } \\
\text { NUMBER }\end{array}$ & $\begin{array}{l}\text { AT II } \\
\text { AGE } \\
\text { CDAYS }\end{array}$ & $\begin{array}{l}\text { JEC TI ON } \\
\text { WE I GHT } \\
\text { (KG) }\end{array}$ & $\begin{array}{r}\text { INJEC TED } \\
(\mu \mathrm{C} / \mathrm{KG})\end{array}$ & IN & $\begin{array}{l}\text { JATE } \\
\text { JECT } \\
\text { MO }\end{array}$ & $\begin{array}{l}\text { TED } \\
Y R\end{array}$ & $\begin{array}{r}\text { DAYS } \\
\text { INJEC } \\
30 / 9 / 64\end{array}$ & $\begin{array}{l}\text { INCE } \\
\text { ION } \\
\text { DEATH }\end{array}$ & $\begin{array}{l}\text { DOSE TO } \\
\text { SKELETO } \\
\text { (RADS) }\end{array}$ \\
\hline 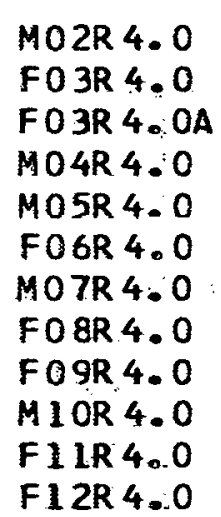 & $\begin{array}{l}470 \\
384 \\
598 \\
408 \\
461 \\
486 \\
453 \\
474 \\
542 \\
527 \\
491 \\
496\end{array}$ & $\begin{array}{c}9.53 \\
8.65 \\
7.20 \\
8.83 \\
13.2 \\
8.55 \\
9.55 \\
8.94 \\
8.53 \\
10.8 \\
10.4 \\
9.61\end{array}$ & $\begin{array}{l}3.55 \\
3.33 \\
3.10 \\
3.47 \\
2.42 \\
3.44 \\
3.88 \\
3.14 \\
3.02 \\
3.28 \\
2.84 \\
2.81\end{array}$ & $\begin{array}{l}16 \\
10 \\
30 \\
7 \\
22 \\
27 \\
24 \\
21 \\
11 \\
27 \\
20 \\
17\end{array}$ & $\begin{array}{l}11 \\
03 \\
11 \\
04 \\
06 \\
07 \\
8 \\
12 \\
04 \\
07 \\
12 \\
01\end{array}$ & $\begin{array}{l}53 \\
54 \\
56 \\
54 \\
54 \\
54 \\
54 \\
54 \\
55 \\
55 \\
55 \\
56\end{array}$ & 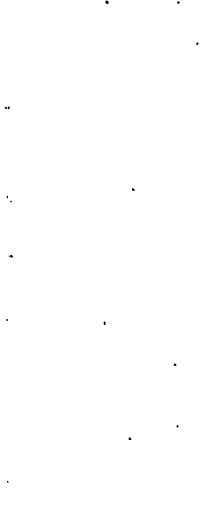 & $\begin{array}{l}1884 \\
490 \\
1614 \\
1518 \\
1659 \\
1939 \\
1647 \\
1324 \\
1471 \\
1553 \\
1469 \\
1435\end{array}$ & $\begin{array}{l}6158 \\
2216 \\
3864 \\
6073 \\
4515 \\
7146 \\
5851 \\
4626 \\
4105 \\
7592 \\
5285 \\
3886\end{array}$ \\
\hline$\ldots \ldots$ & $\ldots \ldots$ & . . .. & $\ldots$. & $\cdots$ & - & . & & 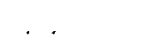 & \\
\hline 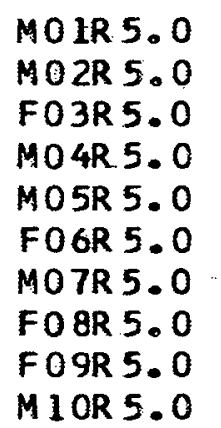 & $\begin{array}{l}473 \\
470 \\
380 \\
408 \\
458 \\
486 \\
453 \\
474 \\
420 \\
527\end{array}$ & $\begin{array}{r}9.87 \\
8.85 \\
7.82 \\
8.90 \\
10.9 \\
9.66 \\
8.85 \\
7.76 \\
9.16 \\
10.7\end{array}$ & $\begin{array}{l}10.5 \\
10.8 \\
10.1 \\
10.6 \\
10.1 \\
10.2 \\
11.9 \\
9.68 \\
9.48 \\
10.2\end{array}$ & $\begin{array}{l}20 \\
16 \\
10 \\
7 \\
22 \\
27 \\
24 \\
21 \\
11 \\
27\end{array}$ & $\begin{array}{r}04 \\
11 \\
03 \\
04 \\
6 \\
07 \\
08 \\
12 \\
04 \\
07\end{array}$ & $\begin{array}{l}53 \\
53 \\
54 \\
54 \\
54 \\
54 \\
54 \\
54 \\
.55 \\
55\end{array}$ & & $\begin{array}{r}908 \\
1380 \\
481 \\
1091 \\
1220 \\
1015 \\
1288 \\
968 \\
1288 \\
825\end{array}$ & $\begin{array}{r}14982 \\
18093 \\
7177 \\
16453 \\
15463 \\
15438 \\
16740 \\
11592 \\
16015 \\
11120\end{array}$ \\
\hline
\end{tabular}


DOG

NUMBER

$M O 2 R 4.0$

FO3R 4.0

FO3R 4 . OA

MO 4R 4.0

MOSR 4.0

FOGR 4.0

MOTR $4: 0$

FO8R 4.0

FO. $4: 0$

M 1 OR 4.0

FIIR 4.0

FI 2R 4.0

MOLR 5.0

MO 2R 5.0

FO3R 5.0

MO 4R 5.0

MOSR 5.0

FO6R 5.0

MOTR 5.0

FO 8R 5.0

FOSR 5.0

M 1OR 5.0
COMMENTS ON DEAD DOES

OSTEOSARCOMA

CANINE DISTENPER

OSTEOSARCOMA

OSTEOSARCOMA

OSTEOSARCONA

OSTEOSARCOMA

OSTEOSARCOMA

OSTEOSARCOMA

OS TEOSARCOMA

OSTEOSARCOMA

OSTEOSARCOMA

OSTEOSARCOMA

DSTEOSARCOMA

OSTEOSARCOMA

CANINE DISTEMPER

DSTEOSARCOMA

OSTEOSARCOMA

O STEDSARCOMA

OSTEOSARCOMA

OSTEOSARCOMA

OSTEOSARCOMA + ANEMI A

OSTEOSARCOMA + FRACTURED MANDIBLE 
B. PLUTONI UM-239

\begin{tabular}{|c|c|c|}
\hline $\begin{array}{l}\text { DOG } \\
\text { NUMB ER }\end{array}$ & $\begin{array}{l}\text { AT IN } \\
\text { AGE } \\
\text { IDAYS }\end{array}$ & $\begin{array}{l}\text { WE IGHT } \\
\text { i) }(K G)\end{array}$ \\
\hline 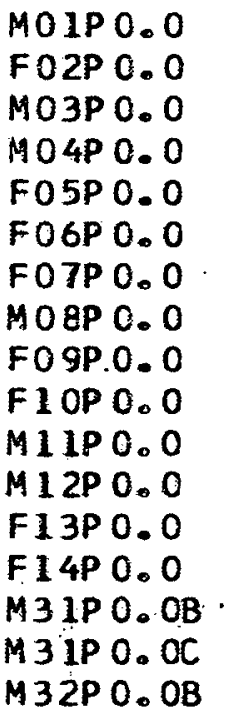 & $\begin{array}{l}443 \\
424 \\
515 \\
426 \\
620 \\
410 \\
515 \\
585 \\
658 \\
658 \\
602 \\
630 \\
517 \\
452 \\
452 \\
452 \\
452\end{array}$ & $\begin{array}{l}0.90 \\
10.9 \\
11.0 \\
11.0 \\
10.3 \\
10.9 \\
9.47 \\
9.89 \\
11.8 \\
12.6 \\
11.2 .\end{array}$ \\
\hline
\end{tabular}

$\begin{array}{lll}\text { DATE } & \text { DAYS SINCE } & \text { DOSE TO } \\ \text { NJECTED } & \text { INJECTION } & \text { SKELETON } \\ \text { D MO YR } & 30 / 9 / 64 \text {. DEATH } & \text { (RADS) }\end{array}$

$\begin{array}{llll}1 & 12 & 52 & 4003 \\ 2 & 03 & 53 & 2755\end{array}$

$1609.53 \quad 4032$

14. $10.53 \quad 4004$

$1205.54 \quad 3794$

$\begin{array}{llll}25 & 10 & 54 & 3628\end{array}$

$\begin{array}{lll}15 & 03.55 & 3487\end{array}$

221155

$221155 \quad 3235$

$24.04 .56 \quad 3081$

$\begin{array}{llll}29 & 05 & 56 & 3046\end{array}$

$-4.364 \quad 210$

$12 \quad 564 \quad 14$

$12 \cdot 564 \quad 141$

12. $564 \quad 141$

12. $564 \quad 141$

3032

$\begin{array}{llclrrrr}\text { F13P0.1 } & 515 & 9.46 & .00068 & 4 & 364 & 210 \\ \text { F14P0.1 } & 452 & 10.3 & .00055 & 12 & 564 & 141 \\ \text { M31P0.1B } & 517 & 12.2 & .00068 & 4: 3.64 & 210\end{array}$

$\begin{array}{llclrrrr}\text { F13P0.2 } & 517 & 9.44 & .00206 & 4: 3.64 & 210 \\ \text { F14P0.2 } & 516 & 7.44 & .00173 & 12 & 564 & 141 \\ \text { M31P0.2B } & 515 & 10.7 & .00185 & 4 & 364 & 210 \\ \text { F31P0.2C } & 452 & 11.9 & .00169 & 12 & 564 & 141 \\ \text { F31P0.2D } & 429 & 9.35 & .00186 & 12 & 564 & 141\end{array}$

$\begin{array}{llclrlrl}\text { F13P0.5 } & 517 & 9.93 & .00540 & 4: 364 & 210 \\ \text { F14P0.5 } & 516 & 9.98 & .00493 & 12 & 564 & 141 \\ \text { M 31P0.5B } & 515 & 10.5 & .00549 & 4: 3.64 & 210\end{array}$

$\begin{array}{llllllll}\text { MOIP1.0 } & 442 & 9.41 & .0150 & 1 & 1252 & 4321 \\ \text { FO2P 1.0 } & 422 & .6 .85 & .0163 & 2 & 03.53 & 4230 \\ \text { MO3P 1.0 } & 515 & 8.00 & .0165 & 1.06 & 53 & 4139\end{array}$


$D O G$

NUMBER

COMMENTS ON DEAD COGS

MOIP 0.0

F02P 0.0

M03P 0.0

$M 04 P 0.0$

F05P 0.0

F06PO. 0

FOTPO. 0

MOQP C. 0

FOSP.0.0

FIOPO.O

MIIP0.0

$M 12 P 0.0$

F13P0. 0

$\mathrm{F} 14 \mathrm{PO} 0.0$

M31PO. OB

MIIPO.OC

M32PO. OB

SPLENIC RUPJURE, METASTATIC SEMINOMA ANESTHETIC ACCIDENT

PULMONARY EMBOLISM, NEPHRITIS

F $13 P 0.1$

F14PO.1

M $31 P 0.1 B$

F $13 P 0.2$

F.14P. 0.2

M31P0.2B

F $31 P 0.2 C$

F 3 IP 0.20

F13P0.5

F1 $4 P 0.5$

M31P.0.5B

MOIP 1:0

F02P 1.0 .

MO3P 1.0 


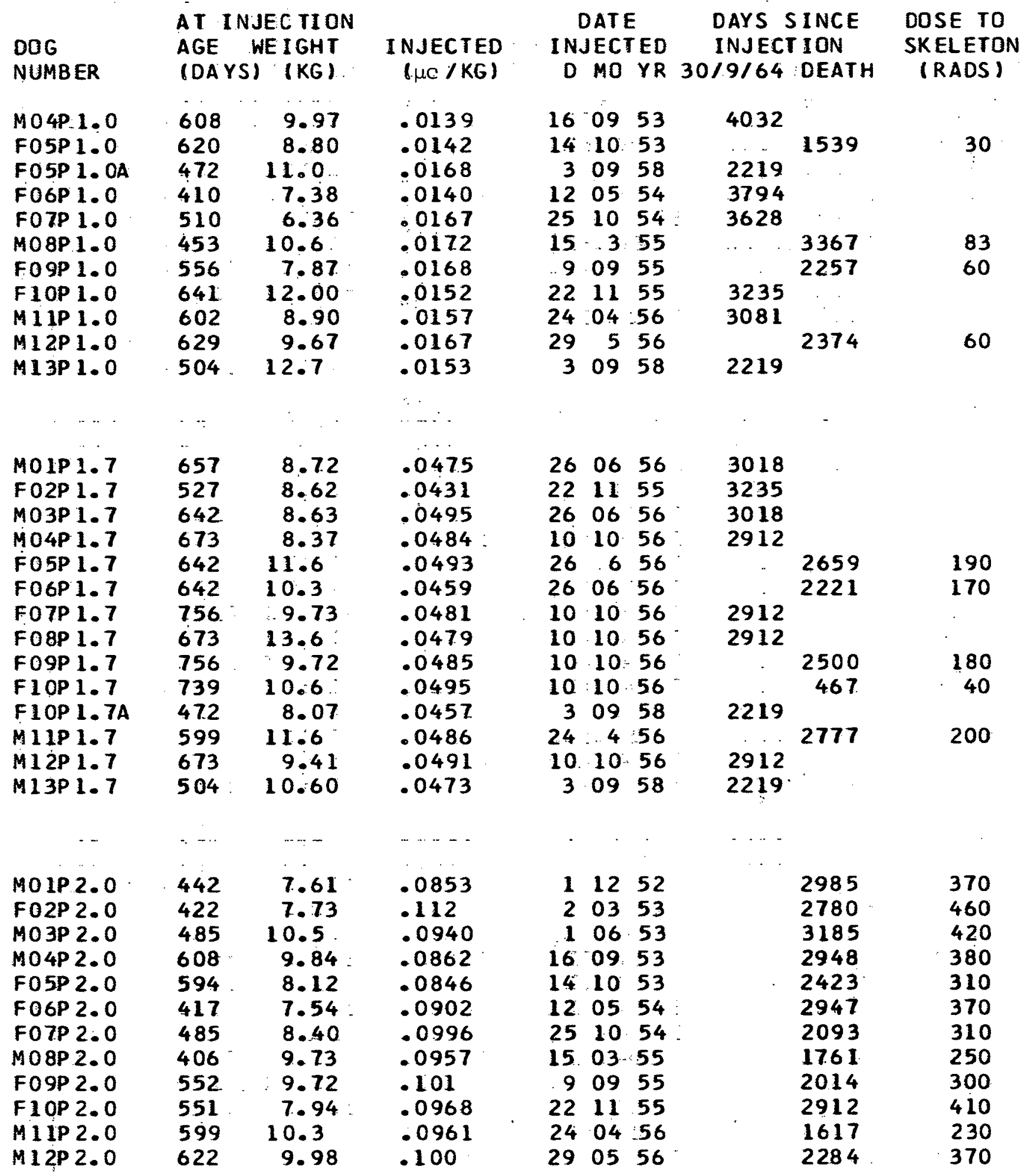


DOG

NUMBER COMMENTS ON DEAC. DOGS

MO4P L. 0 F0.5P 1.0 FOSP 1. OA COLITIS, ENTERITIS * SECCNDARY HEPATIC NECROS IS FO6P 1. 0 F07P 1.0 MO 8P 1.0 FO9P 1.0 F1OP 1.0 $M 11 P 1.0$ M12P1.0 $M 13 P 1.0$

OSTEOSARCOMA OSTEOSARCOMA

CHRONIC PANCREATITIS
MOLIPI.T

FO2P 1.7 MOSP 1.7 MO4P 1. 7 F05P 1.7 FO $6 P 1.7$ FO TP 1.7 F08P 1.7 FO9P 1. 7 F1OP 1.7 F1 OP 1.7A M1IP 1. 7 M 12P 1.7 M $13 P 1.7$

MOLP 2.0 FO2P 2.0 MO3P 2.0 M04P2. 0 FO5P 2.0 F06P 2.0 FO TP 2.0 MO 8P 2.0 FOSP 2.0 F1OP2.0 $M 1 P 2.0$ M12P2.0
O STEOSARCOMA

OSTEOSARCOMA

OSTEOSARCOMA

ACUTE ENTER ITIS

BILE DUCT CARCINOMA

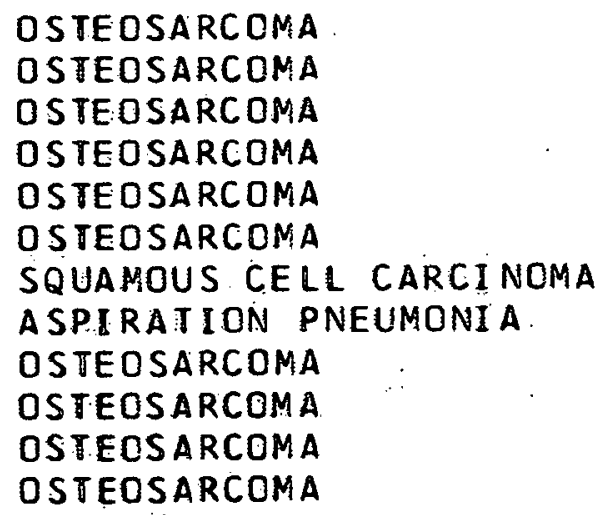




\begin{tabular}{|c|c|c|c|c|c|c|c|c|c|}
\hline $\begin{array}{l}\text { DOG } \\
\text { NUMBER }\end{array}$ & $\begin{array}{l}\text { AT IN } \\
\text { AGE } \\
\text { CDAYS }\end{array}$ & $\begin{array}{l}\text { JEC TION } \\
\text { WE I GHT } \\
\text { (KG) }\end{array}$ & $\begin{array}{r}\text { INJECTED } \\
(\mu C / K G)\end{array}$ & $\begin{array}{c}0 \\
{[N]} \\
D\end{array}$ & $\begin{array}{l}\text { ATE } \\
\text { IECT } \\
\text { MO }\end{array}$ & $\begin{array}{l}\text { E } \\
\text { TED } \\
\text { YR }\end{array}$ & $\begin{array}{r}\text { DAYS } \\
\text { INJEC } \\
30 / 9 / 64\end{array}$ & $\begin{array}{l}5 \text { INCE } \\
\text { ION } \\
\text { DEATH }\end{array}$ & $\begin{array}{l}\text { DOSE TO } \\
\text { SKELETON } \\
\text { (RADS) }\end{array}$ \\
\hline $\begin{array}{l}\text { MO IP } 3.0 \\
F 02 P 3.0 \\
\text { MO3P } 3.0 \\
\text { MO4P } 3.0 \\
F 05 P 3.0 \\
F 06 P 3.0 \\
F 07 P 3.0 \\
\text { MO 8P } 3.0 \\
F 09 P 3.0 \\
F 10 P 3.0 \\
M 11 P 3.0 \\
M 12 P 3.0\end{array}$ & $\begin{array}{l}417 \\
422 \\
485 \\
608 \\
650 \\
415 \\
485 \\
406 \\
552 \\
533 \\
599 \\
622\end{array}$ & $\begin{array}{r}8.00 \\
6.85 \\
8.74 \\
8.51 \\
8.22 \\
8.38 \\
9.00 \\
9.73 \\
7.67 \\
8.94 \\
10.5 \\
10.2\end{array}$ & $\begin{array}{l}.261 \\
.312 \\
.291 \\
.292 \\
.288 \\
.282 \\
.314 \\
.300 \\
.300 \\
.298 \\
.309 \\
.308\end{array}$ & $\begin{array}{r}1 \\
2 \\
1 \\
16 \\
14 \\
12 \\
25 \\
15 \\
9 \\
22 \\
24 \\
29\end{array}$ & $\begin{array}{l}12 \\
03 \\
06 \\
09 \\
10 \\
05 \\
10 \\
03 \\
09 \\
11 \\
04 \\
05\end{array}$ & $\begin{array}{l}52 \\
53 \\
53 \\
53 \\
53 \\
54 \\
54 \\
55 \\
55 \\
55 \\
56 \\
56\end{array}$ & & $\begin{array}{l}1476 \\
1947 \\
1604 \\
1950 \\
1504 \\
1617 \\
1627 \\
1771 \\
1894 \\
1547 \\
1198 \\
1659\end{array}$ & $\begin{array}{l}580 \\
900 \\
700 \\
840 \\
630 \\
670 \\
750 \\
780 \\
840 \\
700 \\
550 \\
760\end{array}$ \\
\hline & $\cdots$ & .... & $\ldots$. & .. & $\cdots$ & - & & $\ldots$ & .. \\
\hline 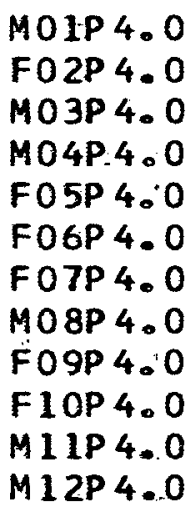 & $\begin{array}{l}442 \\
567 \\
485 \\
566 \\
650 \\
420 \\
485 \\
651 \\
552 \\
527 \\
596 \\
598\end{array}$ & $\begin{array}{r}7.61 \\
8.65 \\
9.36 \\
8.74 \\
7.05 \\
9.26 \\
8.45 \\
9.22 \\
8.58 \\
-6.48 \\
9.56 \\
11.4\end{array}$ & $\begin{array}{r}.823 \\
1.03 \\
.029 \\
.974 \\
.872 \\
.811 \\
.963 \\
.887 \\
.960 \\
.868 \\
.927 \\
.838\end{array}$ & $\begin{array}{l}1 \\
2 \\
1 \\
16 \\
14 \\
12 \\
25 \\
15 \\
.9 \\
22 \\
24 \\
29\end{array}$ & $\begin{array}{l}122 \\
03 \\
06 \\
09 . \\
10 \\
05 \\
10 \\
03 \\
09 \\
11 \\
.04 \\
05\end{array}$ & $\begin{array}{l}52 \\
53 \\
53 \\
53 \\
53 \\
54 \\
54 \\
.55 \\
55 \\
55 \\
.56 \\
56\end{array}$ & & $\begin{array}{l}1724 \\
1556 \\
1198 \\
1066 \\
1245 \\
1357 \\
1198 \\
1157 \\
1343 . \\
1241 \\
1288 \\
1463\end{array}$ & $\begin{array}{l}2100 \\
2380 \\
1680 \\
1560 \\
1650 \\
1660 \\
1730 \\
1560 \\
1920 \\
1660 \\
1790 \\
1840\end{array}$ \\
\hline$\cdots$ & . & 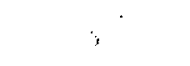 & $\ldots \ldots$ & & - & - & &. & - \\
\hline $\begin{array}{l}\text { MO1P } 5.0 \\
F 02 P 5.0 \\
\text { MO3P } 5.0 \\
\text { MO4P } 5.0 \\
F 05 P 5.0 \\
F 06 P 5.0 \\
F 07 P 5.0 \\
\text { MO8P } 5.0 \\
F 09 P 5.0\end{array}$ & $\begin{array}{r}417 \\
1150 \\
515 \\
566 \\
691 \\
407 \\
482 \\
497 \\
552\end{array}$ & $\begin{array}{r}8.86 \\
8.75 \\
8.10 \\
9.18 \\
8.77 \\
7: 90 \\
8.33 \\
9.55 \\
.9 .45\end{array}$ & $\begin{array}{l}2.67 \\
3.30 \\
3.00 \\
3.17 \\
2.77 \\
2.57 \\
2.99 \\
2.69 \\
2.73\end{array}$ & $\begin{array}{r}1 \\
2 \\
1 \\
16 \\
14 \\
12 \\
25 \\
15 \\
9\end{array}$ & $\begin{array}{l}12 \\
03 \\
06 \\
09 \\
10 \\
05 \\
10 \\
03 \\
09\end{array}$ & $\begin{array}{l}52 \\
53 \\
53 \\
53 \\
53 \\
54 \\
54 \\
-55 \\
55\end{array}$ & . & $\begin{array}{r}1324 \\
1576 \\
499 \\
1562 \\
2059 \\
1194 \\
1491 \\
1192 \\
1145\end{array}$ & $\begin{array}{r}5370 \\
.7830 \\
2340 \\
.7380 \\
8690 \\
4620 \\
6630 \\
4840 \\
4750\end{array}$ \\
\hline
\end{tabular}


DOG

NUMBER

MOIP3.0

F.02P. 3.0

MO3P3. 0

M04P3. 0

F05P3.0

F06P3.0

F07P3.0

MO \&P 3.0

FO9P 3.0

F $10 P 3.0$

A1 IP 3.0

M12P3.0

$M 01 P 4.0$

F02P 4.0 .

MO3P 4.0

MO4P. 4.0

FO5P 4.0

F0 GP 4.0

F07P4.0

MO8P 4.0

F.09P4. 0

F1OP 4 :0

MIIP4. 0

M12P 4.0

MOLP 5.0

F02P5.0

MO3P 5.0

M04P.5. 0

F05P5.0

F06P 5.0

F07P 5.0

MO8P5.O

FOSP 5.0
COMMENTS ON: DEAC DOGS

OSTEOSARCOMA

DSTEOSARCOMA

OSTEOSARCOMA

OSTEOSARCOMA

OSTEOSARCOMA

OSTEOSARCOMA

OSTEOSARCOMA

OSTEOSARCOMA

OSTEOSARCOMA

OSTEOSARCOMA

OSTEOSARCOMA

OSTEOSARCOMA

OSTEOSARCOMA

OSTEOSARCONA

OSTEOSARCOMA

OSTEOSARCOMA

OSTEOSARCOMA

OSTEOSARCOMA

OSTEOSARCOMA

OSTEOSARCOMA

DSTEOSARCOMA

OSTEOSARCOMA

OSTEOSARCOMA

OSTEOSARCOMA
OSTEOSARCOMA

OSTEOSARCOMA + FRACTURED MANDIERE

LIVER DEGENERATION \& ASCITES

OSTEOSARCOMA

OSTEOSARCOMA. LIVER DEGENERATION * HEPATIC HEMORRHAGE

OSTEOSARCOMA

OSTEOSARCOMA \& CRIPPLING FRACTURE

GINGIVITIS

OSTEOSARCOMA, EPISTAXIS A CIRCULATORY COLLAPSE 


\section{RADIUM-228 (MESOTHORIUM) *}

\begin{tabular}{|c|c|c|c|c|c|c|c|c|c|}
\hline $\begin{array}{l}\text { DOG } \\
\text { NUMBER }\end{array}$ & $\begin{array}{l}\text { AT IN } \\
\text { AGE } \\
\text { IDAYS }\end{array}$ & $\begin{array}{l}\text { JEC TI ON } \\
\text { WEI GHT } \\
1 \text { (KG) }\end{array}$ & $\begin{array}{r}\text { I NJECTED } \\
(\mu \mathrm{C} / \mathrm{KG})\end{array}$ & $\begin{array}{l}\text { IN } \\
D\end{array}$ & $\begin{array}{l}\text { ATE } \\
\text { JECT } \\
\text { MO }\end{array}$ & $\begin{array}{l}E \\
\text { TED } \\
Y R\end{array}$ & $\begin{array}{r}\text { DAYS S } \\
\text { INJECT } \\
30 / 9 / 64\end{array}$ & $\begin{array}{l}\text { INCE } \\
\text { ION } \\
\text { DEATH }\end{array}$ & $\begin{array}{l}\text { DOSE TO } \\
\text { SKELETON } \\
\text { (RADS) }\end{array}$ \\
\hline 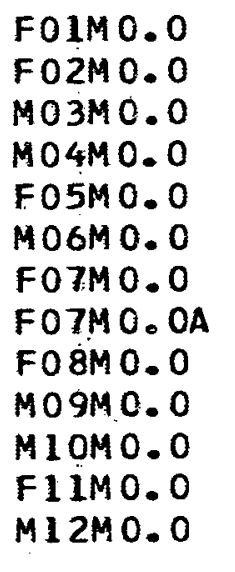 & $\begin{array}{l}732 \\
545 \\
579 \\
601 \\
671 \\
492 \\
395 \\
594 \\
654 \\
575 \\
581 \\
475 \\
695\end{array}$ & $\begin{array}{l}7.33 \\
6.94 \\
13.0 \\
10.3 \\
11.2 \\
.7 .56 \\
8.71 \\
10.9 \\
11.6 \\
12.4 \\
13.3 \\
9.31 \\
10.0\end{array}$ & 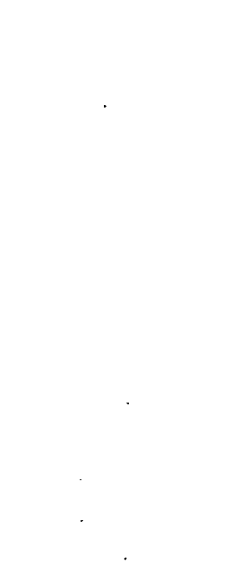 & $\begin{array}{r}4 \\
29 \\
13 \\
15 \\
5 \\
23 \\
4 \\
15 \\
.9 \\
13 \\
17 \\
18 \\
22\end{array}$ & $\begin{array}{r}1 \\
11 \\
03 \\
01 \\
03 \\
04 \\
26 \\
1 \\
03 \\
04 \\
7 \\
9 \\
12\end{array}$ & $\begin{array}{l}54 \\
54 \\
56 \\
57 \\
57 \\
57 \\
57 \\
63 \\
60 \\
60 \\
62 \\
62 \\
60\end{array}$ & $\begin{array}{l}3593 \\
3123 \\
2815 \\
2766 \\
2717 \\
624 \\
1666 \\
1631 \\
806 \\
743 \\
1378\end{array}$ & 3451 & \\
\hline$\cdots$ & $\ldots$ & $\therefore-$ & & $\cdots$ & $\cdots$ & $\cdots$ & & : & \\
\hline 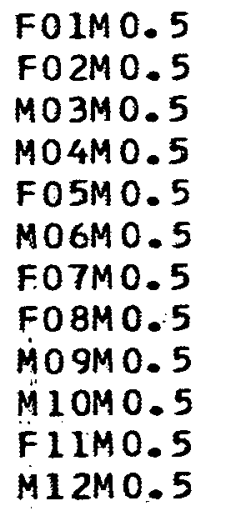 & $\begin{array}{l}492 \\
492 \\
493 \\
475 \\
534 \\
510 \\
492 \\
654 \\
485 \\
492 \\
505 \\
510\end{array}$ & $\begin{array}{c}9.47 \\
9.15 \\
10.8 \\
12.8 \\
.7 .83 \\
10.3 \\
8.87 \\
12.6 \\
11.9 \\
10.6 \\
7.82 \\
10.6\end{array}$ & $\begin{array}{l}.0192 \\
.0189 \\
.0222 \\
.0223 \\
.0188 \\
.0183 \\
.0190 \\
.0191 \\
.0192 \\
.0192 \\
.0224 \\
.01177\end{array}$ & $\begin{array}{l}17 \\
17 \\
18 \\
18 \\
23 \\
23 \\
17 \\
9 \\
13 \\
17 \\
18 \\
23\end{array}$ & $\begin{array}{r}7 \\
7 \\
9 \\
9 \\
10 \\
10 \\
7 \\
03 \\
04 \\
7 \\
9 \\
10\end{array}$ & $\begin{array}{l}62 \\
62 \\
62 \\
62 \\
62 \\
62 \\
62 \\
60 \\
60 \\
62 \\
62 \\
62\end{array}$ & $\begin{array}{l}806 \\
806 \\
743 \\
743 \\
708 \\
708 \\
806 \\
1666 \\
1631 \\
806 \\
743 \\
708\end{array}$ & & \\
\hline 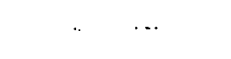 & $\cdots$ & $\ldots$ & $\cdots$ & $*$ & & - & $: \quad-$ & & \\
\hline 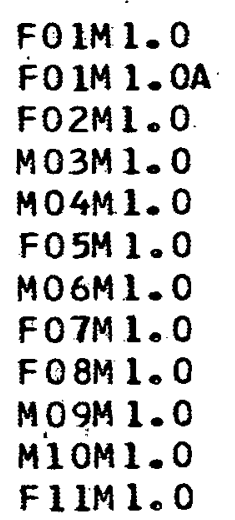 & $\begin{array}{l}718 \\
590 \\
459 \\
575 \\
601 \\
658 \\
521 \\
534 \\
654 \\
485 \\
492 \\
505\end{array}$ & $\begin{array}{r}7.75 \\
8.07 \\
8.25 \\
13.8 \\
9.90 \\
8.80 \\
10.6 \\
9.89 \\
12.4 \\
10.1 \\
9.43 \\
8.91\end{array}$ & $\begin{array}{l}.0561 \\
.0558 \\
.0593 \\
.0574 \\
.0599 \\
.0588 \\
.0545 \\
.0550 \\
.0572 \\
.0582 \\
.0657 \\
.0671\end{array}$ & $\begin{array}{r}4 \\
23 \\
29 \\
13 \\
15 \\
5 \\
23 \\
4 \\
9 \\
13 \\
17 \\
18\end{array}$ & $\begin{array}{r}01 \\
10 \\
11 \\
03 \\
01 \\
03 \\
.04 \\
.6 \\
03 \\
.04 \\
7 \\
9\end{array}$ & $\begin{array}{c}54 \\
62 \\
54 \\
56 \\
57 \\
57 \\
57 \\
57 \\
60 \\
.60 \\
62 \\
62\end{array}$ & $\begin{array}{r}708 \\
3593 \\
3123 \\
2815 \\
2766 \\
2717 \\
1666 \\
1631 \\
806 \\
743\end{array}$ & 2159 & 350 \\
\hline
\end{tabular}


DOG

NUMBER

FOIMO.O F02MO. 0 M03M C. 0 MO4MO.O F05MO.O MO6MC. 0 FOTM0.0 FOTMO.OA FO $8 M 0.0$ MO 9M0.0 MI OM 0.0 FIIMO.O MI 2MO.O

FOMNO. 5 FO2M0. 5 MO $3 M 0.5$ M04MO. 5 F.05MO. 5 AO6MO. 5 F07M0.5 FO8MO. 5 MOSMO. 5 MIOM0. 5 F11M0.5 MI $2 M 0.5$

FOLM1.0 FOIM 1. OA FO2M 1.0 MO3M1.0 MO $4 \mathrm{MI}$. 0 F05M1.0 M06M1.0 FOTM1.O FOEM 1.0 MO9M1.O $M 10 M 1.0$

FIIM1.O
COMMENTS ON DEAD DOES

PURULENT MENI NGOENCEPHAL ITIS

STATUS EPILEPIICUS

HEMANG IOSARCOMA

HEMANGI GSARCOMA 


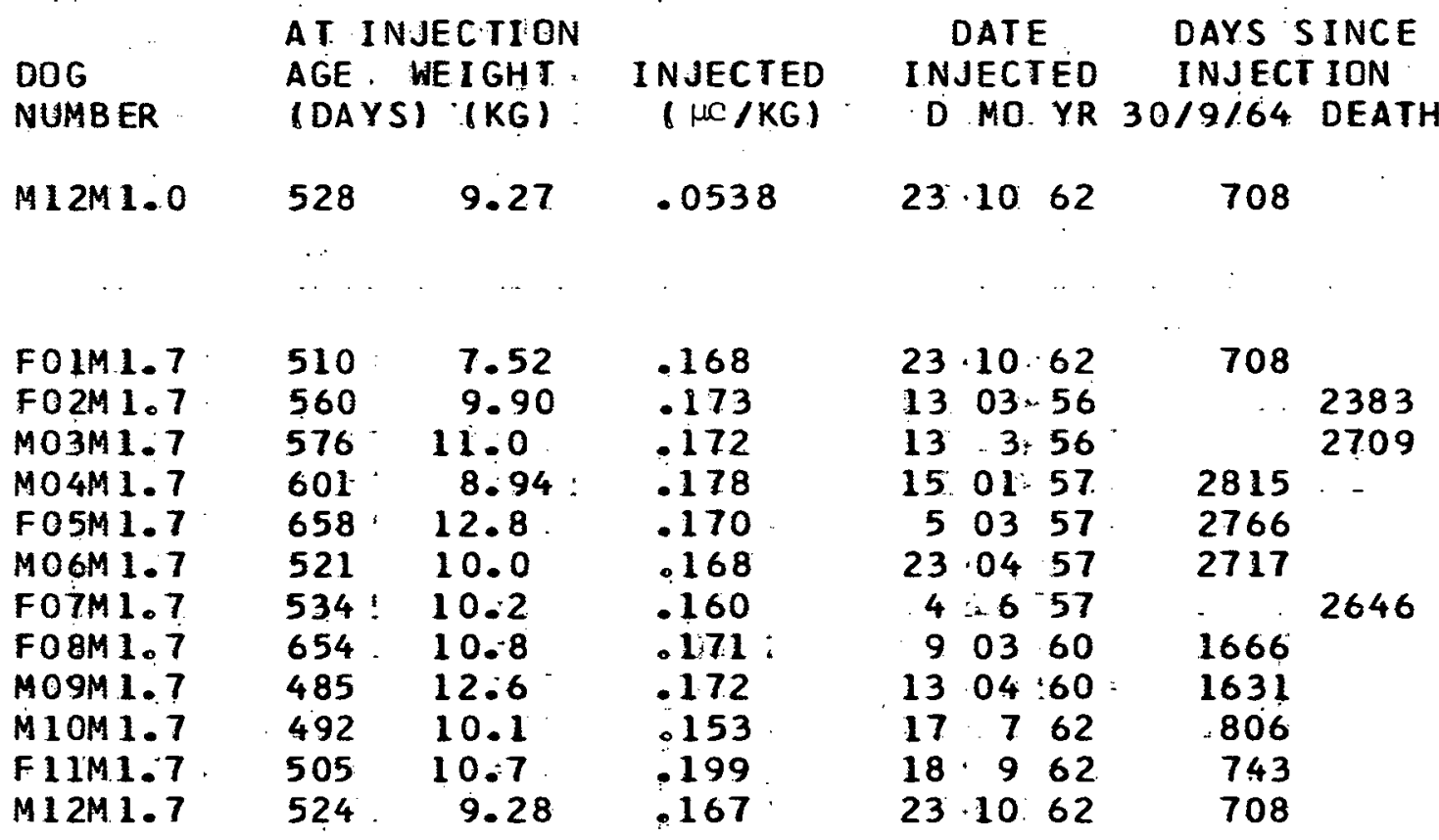

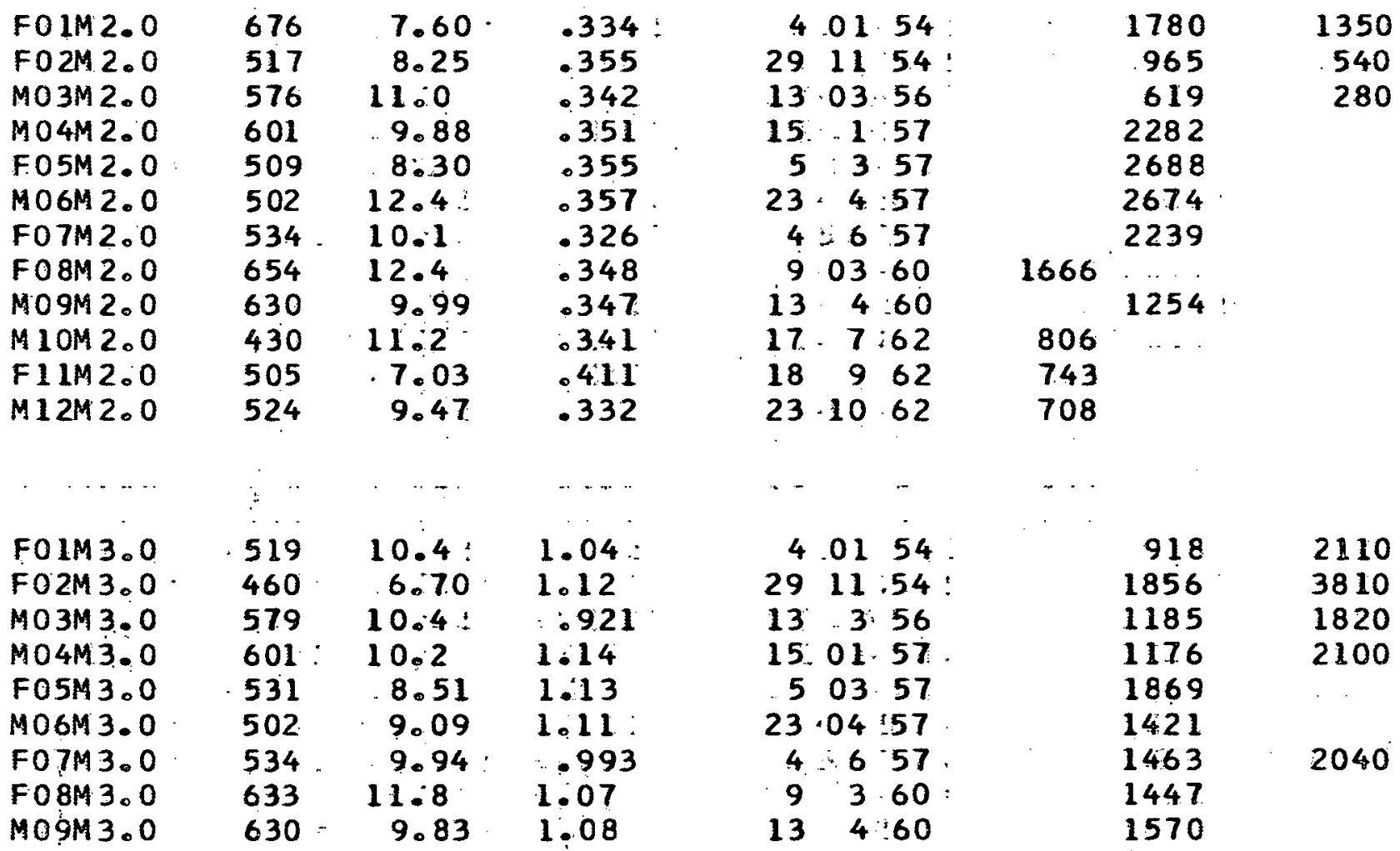


DOG

NUMBER COMMENTS ON DEAD DOGS

M12M1.0

FO1M1.7 F02M 1.7 MO3M1.7 MO4M1.7. F05M 1.7 MO6M1.7 F07M1.7 F08M1.7 MO9M 1.7 M $10 M 1.7$ F11M1.? M12M1.7

OSTEOSARCOMA OSTEOSARCOMA

DSTEOSARCOMA

OSTEOSARCOMA

IN JESTINAL HEMORRHAGE

PNEUMONIA

OSTEOSARCOMA

OSTEOSARCOMA

DSTEOSARCOMA

CHRONIC. PANCREATITIS

REIICULUM CELL SARCOMA OF VERTEBRA
Fo1M3.0 F02M3.O MO3M3.0 MO4M3.0 F05M 3.0 MO6M 3.0 F07M3.0 FO $8 M 3.0$ MOSM 3.0
D STEOSARCOMA

O STE OSARCOMA

OSTEOSARCOMA

OSTEOSARCOMA

O STEOSARCOMA

OSTE OSARCOMA

OSTEOSARCOMA

OSTEOSARCOMA

OSTEOSARCOMA 


\begin{tabular}{|c|c|c|c|c|c|c|c|c|}
\hline $\begin{array}{l}\text { DOG } \\
\text { NUMBER }\end{array}$ & $\begin{array}{l}\text { AT IN } \\
\text { AGE } \\
\text { IDAYS }\end{array}$ & $\begin{array}{l}\text { JECTIDN } \\
\text { WE IGH } \\
\therefore(K G)\end{array}$ & $\begin{array}{l}\text { INJECTED } \\
(\mu C / K G)\end{array}$ & $\begin{array}{l}\text { DAT } \\
\text { I NJECT } \\
\text { D MO }\end{array}$ & $\begin{array}{l}\text { E } \\
\text { TED } \\
Y R\end{array}$ & $\begin{array}{c}\text { DAYS } \\
\text { INJEC } \\
30 / 9.164\end{array}$ & $\begin{array}{l}\text { SINCE } \\
\text { TION } \\
\text { DEATH }\end{array}$ & $\begin{array}{l}\text { DOSE TO } \\
\text { SKELETO } \\
\text { (RADS) }\end{array}$ \\
\hline \multirow[t]{2}{*}{$\begin{array}{l}M 10 M 3.0 \\
F 11 M 3.0 \\
M 12 M 3.0\end{array}$} & $\begin{array}{l}581 \\
499 \\
510\end{array}$ & $\begin{array}{l}10.4 \\
11.0 \\
12.9\end{array}$ & $\begin{array}{l}1.10 \\
1.30 \\
1.05\end{array}$ & $\begin{array}{l}17 \cdot 7 \\
18 \cdot 9 \\
23 \cdot 10\end{array}$ & $\begin{array}{l}62 \\
62 \\
62\end{array}$ & $\begin{array}{l}806 \\
743 \\
708\end{array}$ & & \\
\hline & $\therefore$ & . & $\begin{array}{llll} & \cdots & \cdots\end{array}$ & & & - & $\because$ & \\
\hline $\begin{array}{l}F 01 M 4.0 \\
\text { FO2M4.0 } \\
M O 3 M 4.0 \\
M O 3 M 4.0 A \\
\text { MO 4M4.0 } \\
\text { FO5M4.0 } \\
M 06 M 4.0 \\
\text { FO } 1 M 4.0\end{array}$ & $\begin{array}{l}510 \\
460 \\
579 \\
494 \\
609 \\
509 \\
502 \\
544:\end{array}$ & $\begin{array}{l}7.56 \\
6.95 \\
9.65 \\
7.34 \\
7.84 \\
9.63 \\
9.49 \\
8.40\end{array}$ & $\begin{array}{r}3.15 \\
3.41 \\
3.22 \\
2.89 \\
3.08 \\
3.21 \\
.3 .10 \\
2.92\end{array}$ & $\begin{array}{rr}4 & 01 \\
29 & 11 \\
13 & 03 \\
4 & 06 \\
15 & 1 \\
-5 & 03 \\
23 & 04 \\
4 & 06\end{array}$ & $\begin{array}{l}.54 \\
54 \\
56 \\
.57 \\
57 \\
57 \\
57 \\
57\end{array}$ & & $\begin{array}{r}841 \\
778 \\
418 \\
1063 \\
896 \\
1064 \\
1121 \\
1253\end{array}$ & $\begin{array}{l}5800 \\
3820 \\
1640 \\
4650 \\
3940 \\
5450 \\
5300\end{array}$ \\
\hline$\because \cdot$ & & & $\cdots$ & $\therefore$ & & & $\cdots$ & . \\
\hline $\begin{array}{l}\text { FO1M5.0 } \\
\text { FO2M } 5.0 \\
M 03 M 5.0 \\
M O 4 M 5.0 \\
F O 5 M 5.0 \\
M O 6 M 5.0 \\
F O 7 M 5.0\end{array}$ & $\begin{array}{l}494: \\
460 \\
579 \\
482 \\
658 \\
580 \\
494:\end{array}$ & $\begin{array}{r}7.77 \\
7.35 \\
8.87 \\
7.29 \\
11.1 \\
7.53 \\
7.35\end{array}$ & $\begin{array}{c}9.83 . \\
10.0 \\
9.93 \\
9.82 \\
10.2 \\
10.1 \\
9.77 .\end{array}$ & $\begin{array}{rr}4 & 01 \\
29 & 11 \\
13 & 3 \\
15 & 01 \\
.5 & 3 \\
23 & 4 \\
4 & 06\end{array}$ & $\begin{array}{l}.54 \\
54 \\
56 \\
.57 \\
57 \\
.57 \\
57\end{array}$ & . & $\begin{array}{l}232 \\
780 \\
688 \\
561 \\
770 \\
792 \\
966\end{array}$ & $\begin{array}{r}4130 \\
11200 \\
.9430 \\
6570 \\
10500 \\
10900 \\
14200\end{array}$ \\
\hline
\end{tabular}

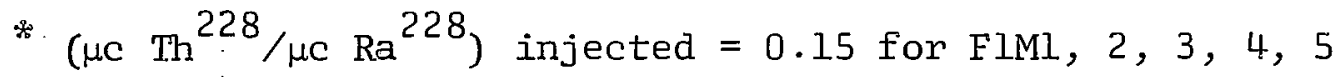

$$
\begin{aligned}
& =0.03 \text { for group } 2,3 \\
& =0.006 \text { for groups } 4,5,6,7,8, \\
& 9,10,11,12, \text { and dogs } \\
& \text { M3M4A, FlMO.5, F2M0.5, } \\
& \text { M3MO.5, FIMIA, and FIMl.7. }
\end{aligned}
$$

$\mathrm{Ra}^{228}$ injected $\mu \mathrm{c} / \mathrm{kg}$ and dose to skeleton values will soon be
revised in accordance with the redetermined half-period of $\mathrm{Ra}^{228}$ (see COO-222 and COO-225) . 
DOG

NUSBER

M $1 O M 3.0$

F $11 M 3.0$

$M 12 M 3.0$

FOIM 4.0

FO2M 4.0

$M O 3 M 4.0$

MO3M4.OA

MO4M4.O

FO5M 4.0

$M O 6 M 4.0$

FO $7 M 4.0$

FOIM 5.0

FO2M 5.0

MO3M5.C

$M O 4 M 5.0$

F05M5.O

MOOM 5.C

FO 7M 5.0
COMMENTS ON DEAC COGS

\section{OSTECSARCOMA + CRIPPLING FRACTURE \\ OSTEOSARCOMA \\ STRANGULATEO INGUINAL HERNIA}

DSTEOSARCCNA, NEPHRITIS, ULCERATIVE GINGIVITIS * PNEUMON FRACTURED MANDIBLE \& ULCERATIVE GINGIVITIS

OSTEOSARCOMA

OSTEOSARCOMA

OSTEOSARCONA

NEPHRITIS * SEVERE ANEMIA

CRIPPLING FRACTURES

ULCERATIVE GINGIVITIS

CRIPPIING FRACTURE

ULCERATIVE GINGIVITIS

OSTEOSARCOMA +CRIPPLING FRACTURE

ULCERATIVE GINGIVITIS, MYOCARDIAL INFARCTICN + GIAUCOMA 
D. THORIUM-228 (RADIOTHOR IUM) *

\begin{tabular}{|c|c|c|c|c|c|c|c|c|c|}
\hline $\begin{array}{l}\text { DOG } \\
\text { NUMB ER }\end{array}$ & $\begin{array}{l}\text { AT IN } \\
\text { AGE } \\
\text { (DAYS) }\end{array}$ & $\begin{array}{l}\text { JEC IION } \\
\text { WEIGHI } \\
\text { J:(KG) }\end{array}$ & $\begin{array}{l}\text { INJECIED } \\
(\mu \mathrm{C} / K G)\end{array}$ & & $\begin{array}{l}\text { JATE } \\
\text { JECI } \\
\text { MO }\end{array}$ & $\begin{array}{l}E \\
\text { TED } \\
\text { YR }\end{array}$ & $\begin{array}{c}\text { DAYS } \\
\text { INJEC } \\
30 / 9 / 64\end{array}$ & $\begin{array}{l}\text { SINCE } \\
\text { TION } \\
\text { DEATH }\end{array}$ & $\begin{array}{l}\text { DOSE TO } \\
\text { SK ELETON } \\
\text { (RADS) }\end{array}$ \\
\hline $\begin{array}{l}\text { MOITO.O } \\
\text { MO2TO.0 } \\
\text { FO3TO.0 } \\
\text { MO4TO.0 } \\
\text { MOSTO.0 } \\
\text { FO6TO.0 } \\
\text { FO6TO.OA } \\
\text { MOTTO.0 } \\
\text { MO8TO.0 } \\
\text { FO9TO.0 } \\
\text { F1OTO.0 } \\
\text { F11TO.0 } \\
\text { F1 } 12 T 0.0\end{array}$ & $\begin{array}{l}4.93 \\
488 \\
797 \\
591 \\
458 \\
489 \\
688 \\
517 \\
533 \\
569 \\
536 \\
530 \\
492\end{array}$ & $\begin{array}{c}8.24 \\
7.28 \\
11.6 \\
8.10 \\
10.4 \\
9.64 \\
8.61 \\
10.5 \\
10.8 \\
8.28 \\
10.4 \\
9.45 \\
9.09\end{array}$ & & $\begin{array}{r}8 \\
28 \\
6 \\
18 \\
14 \\
10 \\
15 \\
-7 \\
24 \\
29 \\
28 \\
4 \\
9\end{array}$ & $\begin{array}{l}02 \\
09 \\
06 \\
10 \\
10 \\
1 \\
12 \\
2 \\
5 \\
6 \\
7 \\
6 \\
7\end{array}$ & $\begin{array}{l}54 \\
54 \\
55 \\
55 \\
58 \\
61 \\
60 \\
61 \\
61 \\
61 \\
61 \\
63 \\
63\end{array}$ & $\begin{array}{r}3887 \\
3655 \\
\\
2178 \\
1385 \\
1331 \\
1225 \\
1189 \\
1160 \\
484 \\
449\end{array}$ & $\begin{array}{r}2592 \\
3072 \\
171\end{array}$ & \\
\hline$\cdots$ & - & $\cdots \cdots$ & & & & & $\because=$ & & \\
\hline $\begin{array}{l}\text { MO1TO.2 } \\
\text { MO2TO.2 } \\
\text { F03TO.2 } \\
\text { M04TO.2 } \\
\text { M05T0.2 } \\
\text { M05TO.2A } \\
\text { F06T0.2 } \\
\text { M07T0.2 } \\
\text { M08T0.2 } \\
\text { F09T0.2 } \\
\text { F10T0.2 } \\
\text { F11T0.2 } \\
\text { F12T0.2 }\end{array}$ & $\begin{array}{l}682 \\
682 \\
478 \\
478 \\
625 \\
530 \\
489 \\
532 \\
494 \\
569 \\
508 \\
530 \\
492\end{array}$ & $\begin{array}{c}11.4 \\
10.4 \\
29.86 \\
10.0 \\
13.8 \\
13.4 \\
8.85 \\
10.5 \\
13.9 \\
7.82 \\
10.5 \\
9.76 \\
7.37\end{array}$ & $\begin{array}{l}.00164 \\
.00166 \\
.00163 \\
.00166 \\
.00162 \\
.00173 \\
.00176 \\
.00159 \\
.00189 \\
.00171 \\
.00170 \\
.00171 \\
.000190\end{array}$ & $\begin{array}{r}27 \\
27 \\
27 \\
27 \\
9 \\
4 \\
10 \\
7 \\
24 \\
29 \\
28 \\
4 \\
9\end{array}$ & $\begin{array}{l}03 \\
03 \\
03 \\
03 \\
02 \\
6 \\
6 \\
1 \\
2 \\
5 \\
6 \\
7 \\
6 \\
7\end{array}$ & $\begin{array}{l}62 \\
62 \\
62 \\
62 \\
60 \\
63 \\
61 \\
61 \\
61 \\
61 \\
61 \\
63 \\
63\end{array}$ & $\begin{array}{r}918 \\
918 \\
918 \\
918 \\
484 \\
1359 \\
1331 \\
1225 \\
1189 \\
1160 \\
484 \\
449\end{array}$ & 889 & 10 \\
\hline$\ldots \ldots$ & & $\ldots$ & & $\ldots$ & $\cdots$ & & $\cdots \cdots$ & & \\
\hline $\begin{array}{l}\text { MO1TO.5 } \\
\text { MO2T0.5 } \\
\text { F03T0.5 } \\
\text { MO4T0.5 } \\
\text { MO5T0.5 } \\
\text { FO6T0.5 } \\
\text { MOZTO.5 } \\
\text { MO8T0.5 } \\
\text { FO9T0.5 } \\
\text { F1OT0.5 } \\
\text { F11T0.5 }\end{array}$ & $\begin{array}{l}699 \\
455 \\
659 \\
516 \\
513 \\
489 \\
532 \\
533 \\
569 \\
508 \\
530\end{array}$ & $\begin{array}{l}14.3 \\
10.5 \\
.8 .59 \\
8.58 \\
8.46 \\
9.66 \\
9.11 \\
9.53 \\
8.62 \\
10.2 \\
7.78\end{array}$ & $\begin{array}{l}.00496 \\
.00490 \\
.00485 \\
.00540 \\
.00522 \\
.00510 \\
.00491 \\
.00562 \\
.00529 \\
.00510 \\
.00518\end{array}$ & $\begin{array}{r}7 \\
28 \\
6 \\
18 \\
14 \\
10 \\
7 \\
24 \\
29 \\
28 \\
4\end{array}$ & $\begin{array}{r}9 \\
09 \\
6 \\
10 \\
10 \\
1 \\
2 \\
5 \\
06 \\
7 \\
6\end{array}$ & $\begin{array}{l}56 \\
54 \\
55 \\
55 \\
58 \\
61 \\
61 \\
61 \\
61 \\
61 \\
63\end{array}$ & $\begin{array}{r}2178 \\
1359 \\
1331 \\
1225 \\
1189 \\
1160 \\
484\end{array}$ & $\begin{array}{l}1976 \\
3032 \\
2159\end{array}$ & $\begin{array}{l}40 \\
40 \\
40\end{array}$ \\
\hline
\end{tabular}


DDG

NUMBER

MOITO. 0

MO2TO. 0

F03T0.0

M04T0. 0

M05TO. 0

F06 0.0

F06TO. OA

MOZTO.O.

MOET 0.0

FO9T0.0

FIOT0.0

F11T0.0

F12T0.0

M01TO. 2

MO2T0.2

F0370.2

MO4TO. 2

M05T0.2

M05TO.2A

F06TO.2

M07T0.2

M08T0.2

Fogro. 2

Flot 0.2

FilTo.2

F12T0.2

MOLTO. 5

M02T0.5

F03ro. 5

M04TO. 5

M05T0. 5

F06 $: 0.5$

MOZJ0.5

M08TO.5

F0970.5

Flot 0.5

F 11 TO. 5

\section{COMMENTS ON DEAD DOGS}

BRAIN HEMORRHAGE

L YMPHOSARCOMA

TRA UMA

STRANGULATION CN YOMITUS + GRAND MAL

\author{
STRANGULATION CN VOMITUS + GRAND MAL \\ PYOMEIRITIS + SECONDARY PERITONITIS \\ STATUS EPILEPTICUS * PNEUMONIA
}




\begin{tabular}{|c|c|c|c|c|c|c|c|c|c|}
\hline $\begin{array}{l}\text { DOG } \\
\text { NUMBER }\end{array}$ & $\begin{array}{l}A T \text { IN } \\
A G E \\
\text { IDAYS }\end{array}$ & $\begin{array}{l}\text { JEC TI ON } \\
\text { WE I GHT } \\
d-(K G)\end{array}$ & $\begin{array}{l}\text { INJECTED } \\
(\mu C / K G)\end{array}$ & $\begin{array}{c}D \\
\text { INJ } \\
D\end{array}$ & $\begin{array}{l}\text { ATE } \\
\text { ECT } \\
\text { MO }\end{array}$ & $\begin{array}{l}\text { E } \\
\text { TED } \\
\text { YR }\end{array}$ & $\begin{array}{r}\text { DAYS } \\
\text { INJEC } \\
30 / 9 / 64\end{array}$ & $\begin{array}{l}\text { S INCE } \\
\text { T ION } \\
\text { DEATH }\end{array}$ & $\begin{array}{l}\text { DOSE TO } \\
\text { SKELETO } \\
\text { (RADS) }\end{array}$ \\
\hline$F 12 \pi 0.5$ & 492 & 9.94 & .00567 & 9 & 7 : & 63 & 449 & & \\
\hline .. & & . & & & & . & & & \\
\hline 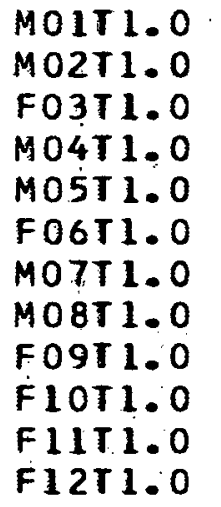 & $\begin{array}{l}493 \\
699 \\
723 \\
699 \\
513 \\
489 \\
532 \\
533 \\
527 \\
508 \\
521 \\
472\end{array}$ & $\begin{array}{c}9.36 \\
9.27 \\
8.84 \\
8.27 \\
11.9 \\
8.81 \\
9.18 \\
8.69 \\
10.0 \\
10.2 \\
7.55 \\
9.96\end{array}$ & $\begin{array}{l}.0146 \\
.0146 \\
.0145 \\
.0146 \\
.0146 \\
.0150 \\
.0147 \\
.0166 \\
.0160 \\
.0150 \\
.0154 \\
.0167\end{array}$ & $\begin{array}{r}8 \\
7 \\
7 \\
7 \\
14 \\
10 \\
7 \\
24 \\
29 \\
28 \\
4 \\
9\end{array}$ & $\begin{array}{l}02 \\
09 \\
09 \\
09 \\
10 \\
1 \\
2 \\
5 \\
6 \\
7 \\
6 \\
7\end{array}$ & $\begin{array}{l}54 \\
56 \\
56 \\
56 \\
58 \\
61 \\
61 \\
61 \\
61 \\
61 \\
-63 \\
63\end{array}$ & $\begin{array}{r}2945 \\
2945 \\
2945 \\
2178 \\
1359 \\
1331 \\
1225 \\
1189 \\
1160 \\
484 \\
449\end{array}$ & 3172 & 120 \\
\hline$\cdots$ & 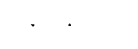 & & $\cdots \cdots$ & .. & $\ldots$ & - & - & & \\
\hline 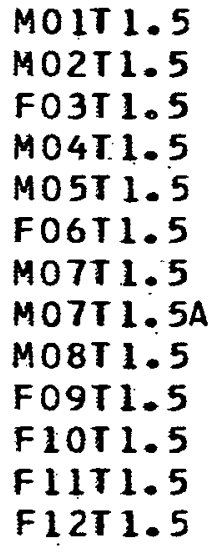 & $\begin{array}{l}699 \\
458 \\
609 \\
591 \\
598 \\
489 \\
517 \\
521 \\
494 \\
527 \\
508 \\
518 \\
465\end{array}$ & $\begin{array}{c}7.95 \\
10.0 \\
10.3 \\
8.59 \\
9.65 \\
8.14 \\
8.83 \\
9.08 \\
11.6 \\
8.80 \\
11.6 \\
11.4 \\
7.56\end{array}$ & $\begin{array}{l}.0289 \\
.0293 \\
.0303 \\
.0299 \\
.0286 \\
.0292 \\
.0292 \\
.0311 \\
.0324 \\
.0306 \\
.0296 \\
.0305 \\
.0329\end{array}$ & $\begin{array}{r}7 \\
28 \\
6 \\
18 \\
9 \\
10 \\
7 \\
4 \\
24 \\
29 \\
28 \\
4 \\
9\end{array}$ & $\begin{array}{l}9 \\
09 \\
06 \\
10 \\
2 \\
1 \\
2 \\
6 \\
5 \\
6 \\
7 \\
6 \\
7\end{array}$ & $\begin{array}{l}56 \\
54 \\
55 \\
55 \\
60 \\
61 \\
61 \\
63 \\
61 \\
61 \\
61 \\
63 \\
63\end{array}$ & $\begin{array}{r}1359 \\
484 \\
1225 \\
1189 \\
1160 \\
484 \\
449\end{array}$ & $\begin{array}{l}2894 \\
2576 \\
1921 \\
2309 \\
1624 \\
384 \\
\end{array}$ & $\begin{array}{r}231 \\
230 \\
220 \\
230 \\
194 \\
80\end{array}$ \\
\hline .. & $\ldots$ & … - & $\ldots \ldots$ & & & 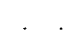 & & & \\
\hline $\begin{array}{l}\text { MOIT2.0 } \\
\text { MO2T } 2.0 \\
F 03 T 2.0 \\
\text { MO4T } 2.0 \\
\text { MO4T2.0A } \\
\text { MO5T } 2.0 \\
\text { FO TT } 2.0 \\
M O T T 2.0\end{array}$ & $\begin{array}{l}491 \\
483 \\
474 \\
553 \\
650 \\
598 \\
451 \\
517\end{array}$ & $\begin{array}{l}10.2 \\
9.16 \\
7.87 \\
13.0 \\
10.6 \\
9.12 \\
8.65 \\
8.85\end{array}$ & $\begin{array}{l}.0976 \\
.0875 \\
.0908 \\
.0900 \\
.0899 \\
.0848 \\
.0879 \\
.0881\end{array}$ & $\begin{array}{r}8 \\
28 \\
6 \\
18 \\
7 \\
9 \\
10 \\
7\end{array}$ & $\begin{array}{l}02 \\
09 \\
06 \\
10 \\
09 \\
02 \\
1 \\
2\end{array}$ & $\begin{array}{l}54 \\
54 \\
55 \\
55 \\
56 \\
60 \\
61 \\
61\end{array}$ & & $\begin{array}{r}1282 \\
1234 \\
1541 \\
78 \\
1222 \\
1085 \\
1108 \\
1015\end{array}$ & $\begin{array}{l}580 \\
540 \\
610 \\
60 \\
540 \\
480 \\
500 \\
480\end{array}$ \\
\hline
\end{tabular}


DOG

NUABEER

$F 1270.5$

MO1T 1.0

$M 02 T 1: 0$

F03T1.0

M04T1.0

MO5T 1.0

F06T1.0

A 0711.0

MOET 1.0

FO9T 1.0

F10T1.0

FIIT1.0

F $12 T 1.0$

$M 01 T 1.5$

M02T 1.5

FO3T1.5

M04T1. 5

M05T1.5

F06T 1.5

M07T L. 5

M07T 1. 5A

MOET 1.5

F09T1.5

F10T1.5

F11T1.5

F12T1.5

MolT2. 0

MO2T 2.0

F03T2.O

MO4T2.O

MO4T2.OA

MO5T2.O

F06T 2.0

M07T2.O
COMMENTS ON DEAD DOGS

OSTEOSARCOMA
OSTEOSARCOMA

OS JEÓSARCOMA

OSTEOSARCOQMA

IRAUMA

DSTEOSARCOMA

DSTEOSARCOMA

OSTEOSARCOMA

OSTEOSARCOMA 


\begin{tabular}{|c|c|c|c|c|c|c|c|c|c|}
\hline $\begin{array}{l}\text { DOG } \\
\text { NUMBER }\end{array}$ & $\begin{array}{l}\text { AT IN } \\
\text { AGE } \\
\text { (DAYS) }\end{array}$ & $\begin{array}{l}\text { JEC TION } \\
\text { WE IGHT } \\
\text { (KG) }\end{array}$ & $\begin{array}{l}\text { I N JEC TED } \\
(\mu \mathrm{C} / \mathrm{KG})\end{array}$ & $\begin{array}{c}\text { IN } \\
\text { D }\end{array}$ & $\begin{array}{l}\text { ATE } \\
\text { MECT } \\
\text { MO }\end{array}$ & $\begin{array}{l}\text { TED } \\
\text { YR }\end{array}$ & $\begin{array}{r}\text { DAYS } \\
\text { INJECI } \\
30 / 9 / 64\end{array}$ & $\begin{array}{l}\text { SINCE } \\
\text { ION } \\
\text { DEATH }\end{array}$ & $\begin{array}{l}\text { DOSE TO } \\
\text { SKELETON } \\
\text { (RADS) }\end{array}$ \\
\hline $\begin{array}{l}\text { MO8T } 2.0 \\
F 09 T 2.0 \\
F 10 T 2.0 \\
F 11 T 2.0 \\
F 12 T 2.0\end{array}$ & $\begin{array}{l}533 \\
527 \\
508 \\
518 \\
464\end{array}$ & $\begin{array}{l}10.7 \\
8.09 \\
10.7 \\
10.8 \\
8.92\end{array}$ & $\begin{array}{l}.0981 \\
.0979 \\
.0919 \\
.0904 \\
.100\end{array}$ & $\begin{array}{r}24 \\
29 \\
28 \\
4 \\
9\end{array}$ & $\begin{array}{l}5 \\
6 \\
7 \\
6 \\
7\end{array}$ & $\begin{array}{l}61 \\
61 \\
61 \\
63 \\
63\end{array}$ & $\begin{array}{r}1189 \\
484 \\
449\end{array}$ & $\begin{array}{l}1078 \\
1022\end{array}$ & $\begin{array}{l}548 r \\
498\end{array}$ \\
\hline & $\cdots$ & & - & 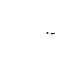 & & & & & \\
\hline $\begin{array}{l}\text { MOIT } 3.0 \\
\text { MO2T } 3.0 \\
F 03 T 3.0 \\
\text { MO4T } 3.0 \\
\text { MO5T } 3.0 \\
F O 6 T 3.0 \\
\text { MOTT } 3.0 \\
\text { MO8T } 3.0 \\
F 09 T 3.0 \\
F 10 T 3.0 \\
F 11 T 3.0 \\
F 12 T 3.0\end{array}$ & $\begin{array}{l}314 \\
458 \\
471 \\
606 \\
571 \\
451 \\
427 \\
494 \\
511 \\
508 \\
518 \\
459\end{array}$ & $\begin{array}{l}9.15 \\
11.9 \\
12.0 \\
9.69 \\
10.7 \\
8.83 \\
9.90 \\
10.1 \\
11 . .5 \\
9.26 \\
10.3 \\
11.5\end{array}$ & $\begin{array}{l}.301 \\
.301 \\
.272 \\
.285 \\
.269 \\
.282 \\
.266 \\
.313 \\
.298 \\
.280 \\
.290 \\
.320\end{array}$ & $\begin{array}{r}8 \\
28 \\
6 \\
18 \\
9 \\
10 \\
7 \\
24 \\
29 \\
28 \\
4 \\
9\end{array}$ & $\begin{array}{l}02 \\
09 \\
06 \\
10 \\
02 \\
1 \\
2 \\
5 \\
6 \\
7 \\
6 \\
7\end{array}$ & $\begin{array}{l}54 \\
54 \\
55 \\
55 \\
60 \\
61 \\
61 \\
61 \\
61 \\
61 \\
63 \\
63\end{array}$ & $\begin{array}{l}484 \\
449\end{array}$ & $\begin{array}{r}988 \\
859 \\
547 \\
801 \\
890 \\
1156 \\
861 \\
685 \\
1062 \\
971\end{array}$ & $\begin{array}{r}1590 \\
1400 \\
1080 \\
1390 \\
1340 \\
1640 \\
1300 \\
1320 \\
\because 1651 \\
1470\end{array}$ \\
\hline$\cdot \quad \ldots .$. & . & .. & & - & & ... & & & \\
\hline $\begin{array}{l}M 01 T 4.0 \\
M 02 T 4.0 \\
F O 3 T 4.0 \\
M O 4 T 4.0\end{array}$ & $\begin{array}{l}480 \\
458 \\
461 \\
606\end{array}$ & $\begin{array}{l}8.32 \\
8.32 \\
7.25 \\
8.81\end{array}$ & $\begin{array}{l}.882 \\
.916 \\
.800 \\
.835\end{array}$ & $\begin{array}{r}8 \\
28 \\
6 \\
18\end{array}$ & $\begin{array}{l}02 \\
09 \\
06 \\
10\end{array}$ & $\begin{array}{l}54 \\
54 \\
55 \\
55\end{array}$ & & $\begin{array}{l}645 \\
833 \\
763 \\
793\end{array}$ & $\begin{array}{l}3400 \\
4330 \\
4250 \\
4110\end{array}$ \\
\hline$\cdots$ & $\ldots$ & $\ldots$ & . $\quad-$ & . & &. & & . & \\
\hline $\begin{array}{l}\text { MO1T5.0 } \\
\text { MO2T } 5.0\end{array}$ & $\begin{array}{l}480 \\
483\end{array}$ & $\begin{array}{l}9.48 \\
8.22\end{array}$ & $\begin{array}{l}2.76 \\
2.63\end{array}$ & $\begin{array}{r}8 \\
28\end{array}$ & $\begin{array}{l}02 \\
09\end{array}$ & $\begin{array}{l}54 \\
54\end{array}$ & & $\begin{array}{r}212 \\
97\end{array}$ & $\begin{array}{l}4810 \\
2080\end{array}$ \\
\hline
\end{tabular}


DOG

NUMBER

Mogt 2.0

F097 2.0

F10T2.0

F11T2.0

F $12 \mathrm{~T} 2.0$

$M 01 T 3.0$

MO2T.3.O

$\mathrm{FO} 3 \mathrm{T3} . \mathrm{O}$

$M 04 T 3.0$

0513.0

FO6T 3.0

MOTT3.0

MO8T3.0

F0973.0

F1OT 3.0

FIII3.0

F12 12.0

$M 01 T 4.0$

$M 0214.0$

FO3T4.0

MO $4 \mathrm{~T} 4.0$

MO1T5.0

MO2T 5.0
COAMENTS ON DEAD DOGS

OSTEOSARCOMA

OSTEOSARCOMA

OSTEOSARCOMA + SEVERE ANEMIA

OSTEOSARCOMA * TRAUMA

OSTEOSARCOMA

OSTEOSARCOMA

OSTEOSARCOMA

OSTEOSARCOMA

OSTECSARCOMA

OSTEOSARCON:A

OSTEOSARCOMA

OSTEOSARCOMA
OSTEOSARCOMA + CRIPPLING FRACTURE OSTEOSARCOMA. CRI PPLING FRACTURE + NEPHRITIS ULCERATIVE GINGIVITIS + NEPHRITIS ULCERATIVE GINGIVITIS

NEPHRITIS

PANC YTOPENIA 
E. STRONTI UM-90

\begin{tabular}{|c|c|c|c|c|c|c|c|c|c|}
\hline $\begin{array}{l}\text { DOG } \\
\text { NUMBER }\end{array}$ & $\begin{array}{l}\text { AT I } \\
\text { AGE } \\
\text { IDAY }\end{array}$ & $\begin{array}{l}\text { JEC TION } \\
\text { WE I GHT } \\
\because(\mathrm{KG})\end{array}$ & $\begin{array}{c}\text { I NJEC TED } \\
(\mu \mathrm{K} / \mathrm{KG})\end{array}$ & I & $\begin{array}{l}\text { DATE } \\
\text { JECT } \\
\text { MO }\end{array}$ & $\begin{array}{l}E \\
\text { TED } \\
Y R\end{array}$ & $\begin{array}{c}\text { DAYS } \\
\text { INJECI } \\
30 / 9164\end{array}$ & $\begin{array}{l}\text { S INCE } \\
\text { T ION } \\
\text { DEATH }\end{array}$ & $\begin{array}{l}\text { DOSE TO } \\
\text { SKELETON } \\
\text { (RADS) }\end{array}$ \\
\hline $\begin{array}{l}F 01 S 0.0 \\
M 02 S 0.0 \\
M 03 S 0.0 \\
F 04 S 0.0 \\
M 05 S 0.0 \\
M 06 S 0.0 \\
F 07 S 0.0 A \\
F 0850.0 \\
F 09 S 0.0 \\
F 09 S 0.0 A \\
M 1 O S 0.0 \\
F 11 S 0.0 \\
M 12 S 0.0\end{array}$ & $\begin{array}{l}502 \\
600 \\
493 \\
520 \\
542 \\
466 \\
462 \\
483 \\
549 \\
535 \\
522 \\
541 \\
605\end{array}$ & $\begin{array}{c}8.48 \\
11.1 \\
9.03 \\
8.19 \\
10.6 \\
9.68 \\
9.46 \\
9.29 \\
12.4: \\
11.2 \\
13.9 \\
9.60 \\
8.99\end{array}$ & $"$ & $\begin{array}{l}18 \\
14 \\
11 \\
15 \\
19 \\
27 \\
7 \\
19 \\
11 \\
4 \\
29 \\
3 \\
6\end{array}$ & $\begin{array}{l}01 \\
002 \\
09 \\
10 \\
11 \\
05 \\
01 \\
05 \\
08 \\
26 \\
09 \\
11 \\
01\end{array}$ & $\begin{array}{l}55 \\
56 \\
57 \\
57 \\
57 \\
58 \\
.59 \\
59 \\
59 \\
63 \\
59 \\
59 \\
60\end{array}$ & $\begin{array}{r}3543 \\
3151 \\
2576 \\
2542 \\
2507 \\
2318 \\
2093 \\
1961 \\
484 \\
1828 \\
1793 \\
1729\end{array}$ & 708 & \\
\hline$\ldots$ & $\because \cdots$ & $\ldots$ & & $\ldots$ & & · " & $\therefore$ & & \\
\hline $\begin{array}{l}\text { F01S1.0 } \\
F 01 S 1.0 A \\
M 02 S 1.0 \\
M 03 S 1.0 \\
F 04 S 1.0 \\
M 05 S 1.0 \\
M 0651.0 \\
F 07 S 1.0 \\
F 08 S 1.0 \\
F 0951.0 \\
M 10 S 1.0 \\
F 11 S 1.0 \\
M 12 S 1.0 \\
\end{array}$ & $\begin{array}{c}1524: \\
521 \\
567 \\
493 \\
525 \\
555 \\
466 \\
524 \\
483 \\
549 \\
522 \\
543 \\
607\end{array}$ & $\begin{array}{c}6.84 \\
9.38 \\
8.81 \\
10.9 \\
8.96 \\
10.2 \\
9.56 \\
9.94 \\
10.8 \\
11.6 \\
11.5 \\
10.3 \\
13.7\end{array}$ & $\begin{array}{l}.573 \\
.588 \\
.606 \\
.572 \\
.560 \\
.532 \\
.581 \\
.517 \\
.697 \\
.534 \\
.558 \\
.550 \\
.559\end{array}$ & $\begin{array}{l}18 \\
14 \\
14 \\
11 \\
15 \\
19 \\
27 \\
11 \\
19 \\
11 . \\
29 \\
3 \\
6\end{array}$ & $\begin{array}{l}01 \\
02 \\
02 \\
09 \\
10 \\
11 \\
.05 \\
11 \\
05 \\
08 \\
09 \\
11 \\
01\end{array}$ & $\begin{array}{l}55 \\
56 \\
56 \\
57 \\
57 \\
57 \\
58 \\
58 \\
59 \\
59 \\
59 \\
59 \\
60\end{array}$ & $\begin{array}{l}3151 \\
3151 \\
2576 \\
2542 \\
2507 \\
2318 \\
2150 \\
1961 \\
1877 \\
1828 \\
1793 \\
1729\end{array}$ & 308 & 20 \\
\hline 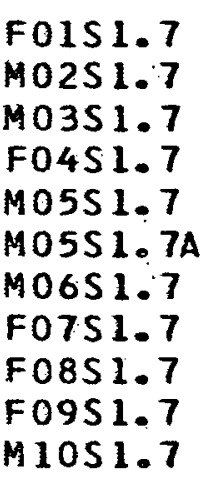 & $\begin{array}{l}526 \\
567 \\
493 \\
522 \\
560 \\
493 \\
466 \\
488 \\
472 \\
549 \\
519\end{array}$ & $\begin{array}{c}7.41 \\
11.6 \\
9.19 \\
9.60 \\
9.85 \\
11.4 \\
10.6 \\
10.2 \\
8.47 \\
10.0 \\
13.6\end{array}$ & $\begin{array}{l}1.78 \\
1.84 \\
1.69 \\
1.68 \\
1.60 \\
1.78 \\
1.72 \\
1.60 \\
2.03 \\
1.62 \\
1.66\end{array}$ & $\begin{array}{l}14 \\
14 \\
11 \\
15 \\
19 \\
.6 \\
27 \\
11 \\
19 \\
11 \\
29\end{array}$ & $\begin{array}{l}02 . \\
02 \\
09 \\
10 \\
11 \\
3 \\
05 \\
11 . \\
5 \\
08 \\
09\end{array}$ & $\begin{array}{l}56 \\
56 \\
57 \\
57 \\
57 \\
63 \\
58 \\
58 \\
59 \\
59 \\
59\end{array}$ & $\begin{array}{l}3151 \\
3151 \\
2576 \\
2542 \\
5 \\
574 \\
2318 \\
2150 \\
1877 \\
1828\end{array}$ & $\begin{array}{c}1715 \\
1973\end{array}$ & 152 \\
\hline
\end{tabular}


DOG

NUMBER

Folso.o

M0250.0

M0350. 0

F0450.0

M05SO.O

M06SO.0

F0750.0A

F08SO.O

FOSSO.0

F0.950.0A

MIOSO.0

F11S0.0

$M 1250.0$

F01S1.0

FO1S1. OA

$M 02 S 1.0$

M03S1. 0

F0451.0

MO5S1.0

M06S1.0

F0751.0

F08S1.0

FOOS1. 0

M 1051.0

F 1151.0

M1251.0

F01S 1.7

MO2S1.?

M0351.7

F04S1.7

MO5Si:7

M0551.7A

M06S1.7

F07.S1.7

Fo8S 1.7

F0951.7

M1OS1.7

\section{COMMENTS ON DEAD DOES}

TRAUMA

SACRIFICED -IMPROPER INJECTION AGE-

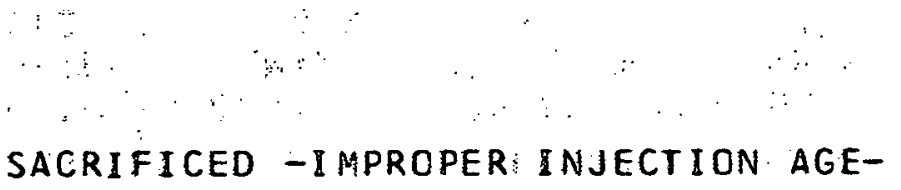

COMA OF UNKNOWN ETIOLOGY

STATUS EPILEPTICUS, CHRONIC PANCREATITIS 


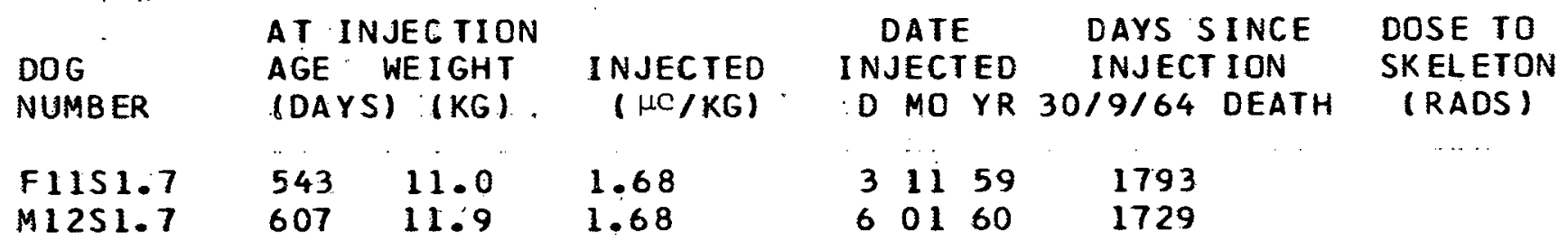

\begin{tabular}{|c|c|c|c|c|c|c|c|c|c|}
\hline $\begin{array}{l}F 01 S 2.0 \\
M O 2 S 2.0 \\
M 03 S 2.0 \\
F O 4 S 2.0 \\
M 05 S 2.0 \\
M 06 S 2.0 \\
F 07 S 2.0 \\
F 08 S 2.0 \\
F 09 S 2.0 \\
M 1 O S 2.0 \\
F 11 S 2.0 \\
M 12 S 2.0\end{array}$ & $\begin{array}{l}502 \\
567 \\
494 \\
522 \\
560 \\
466 \\
488 \\
465 \\
473 \\
508 \\
543 \\
607\end{array}$ & $\begin{array}{r}5.59 \\
8.97 \\
7.82 \\
9.68 \\
8.72 \\
9.19 \\
11.2 \\
9.49 \\
14.1 \\
10.7 \\
10.4 \\
11.6\end{array}$ & $\begin{array}{l}3.70 \\
3.42 \\
3.39 \\
3.41 \\
3.24 \\
3.50 \\
3.19 \\
4.14 \\
3.28 \\
3.34 \\
3.41 \\
3.49\end{array}$ & $\begin{array}{l}18 \\
14 \\
11 \\
15 \\
19 \\
27 \\
11 \\
19 \\
11 \\
29 \\
3 \\
6\end{array}$ & $\begin{array}{l}1 \\
02 \\
09 \\
10 \\
11 . \\
05 \\
11 \\
05 \\
08 \\
09 \\
11 \\
01\end{array}$ & $\begin{array}{l}55 \\
56 \\
57 \\
57 \\
57 \\
58 \\
58 \\
59 \\
59 \\
59 \\
59 \\
60\end{array}$ & $\begin{array}{l}3151 \\
2576 \\
2542 \\
2507 \\
2318 \\
2150 \\
1961 \\
1877 \\
1828 \\
1793 \\
1729\end{array}$ & 3269 & 451 \\
\hline$\cdots$ & & & $\ldots \ldots$ & - & - & .. & & & \\
\hline $\begin{array}{l}F 01 S 3.0 \\
M O 2 S 3.0 \\
M O 3 S 3.0 \\
F 04 S 3.0 \\
M O S S 3.0 \\
M O 6 S 3.0 \\
F O 7 S 3.0 \\
F O 8 S 3.0 \\
F O 9 S 3.0 \\
M 1 O S 3.0 \\
F 11 S 3.0 \\
M 12 S 3.0\end{array}$ & $\begin{array}{l}468 \\
565 \\
494 \\
527 \\
557 \\
466 \\
486 \\
465 \\
468 \\
519 \\
541 \\
605\end{array}$ & $\begin{array}{r}7.36 \\
9.62 \\
11.4 . \\
9.17 \\
8.90 \\
9.44 \\
9.80 \\
12.5 \\
10.0 \\
12.5 \\
9.00 \\
8.43\end{array}$ & $\begin{array}{l}11.6 \\
11.6 \\
10.8 \\
10.6 \\
10.1 \\
10.9 \\
10.1 \\
12.9 \\
10.1 \\
10.3 \\
10.8 \\
10.2\end{array}$ & $\begin{array}{l}18 \\
14 \\
11 \\
15 \\
19 \\
27 \\
11 \\
19 \\
11 \\
29 \\
3 \\
6\end{array}$ & $\begin{array}{l}01 \\
02 \\
09 \\
10 \\
11 \\
05 \\
11 \\
05 \\
08 \\
09 \\
11 \\
01\end{array}$ & $\begin{array}{l}55 \\
56 \\
57 \\
57 \\
57 \\
58 \\
58 \\
59 \\
59 \\
59 \\
59 \\
.60\end{array}$ & $\begin{array}{l}3543 \\
3151 \\
2576 \\
2542 \\
2507 \\
2318 \\
2150 \\
1961 \\
1877 \\
1828 \\
1793 \\
1729\end{array}$ & & \\
\hline$\ldots$ & $\cdots$ & & $\cdots$ & $\because$ & $\cdots$ & & - & & \\
\hline $\begin{array}{l}F 01 S 4.0 \\
M 02 S 4.0 \\
M O 3 S 4.0 \\
F 04 S 4.0 \\
M O 5 S 4.0 \\
M 06 S 4.0 \\
F 0754.0 \\
F 08 S 4.0\end{array}$ & $\begin{array}{l}468 \\
567 \\
593 \\
528 \\
562 \\
504 \\
478 \\
465\end{array}$ & $\begin{array}{c}8.7 .4 \\
11.2 \\
9.83 \\
8.24 \\
9.65 \\
16.0 \\
10.9 \\
10.9\end{array}$ & $\begin{array}{l}33.3 \\
32.6 \\
32.1 \\
32.1 \\
30.6 \\
32.7 \\
30.9 \\
40.6\end{array}$ & $\begin{array}{l}18 \\
14 \\
11 \\
15 \\
19 \\
.3 \\
11 \\
19\end{array}$ & $\begin{array}{l}01 \\
02 \\
09 \\
10 \\
11 \\
09 \\
11 \\
05\end{array}$ & $\begin{array}{l}55 \\
56 \\
57 \\
57 \\
57 \\
58 \\
58 \\
59\end{array}$ & $\begin{array}{l}3543 \\
2576 \\
2542 \\
2507 \\
2219 \\
2150 \\
1961\end{array}$ & 2093 & 5464 \\
\hline
\end{tabular}


DOG

NUMBER

F1IS1.7 M12S1.7

Fo152.0 $M 0252.0$ M03S2.O F0 452.0 MOSS2.0 MO6S2.0 F07.52.0 FO8S2. 0 F0952.0 $M 1052.0$ F1152.0 12S2. 0

Fo1S 3.0 Mo253.0 M03S3.0 F04S3.0 M05S3.0 M0653.0 F0753.0 FO 853.0 F.OSS 3.0 M 1053.0 F11S3.0 $\mathrm{M} 12 \mathrm{S3} .0$

F0154.0 MO2S4:0 M03S4. 0 F04S4.0 MOSS4. 0 M0654.0 F07S4. 0 F.0854.0
COMMENTS ON DEAD DOGS

\section{BACTERIAL PNEUMONIA}

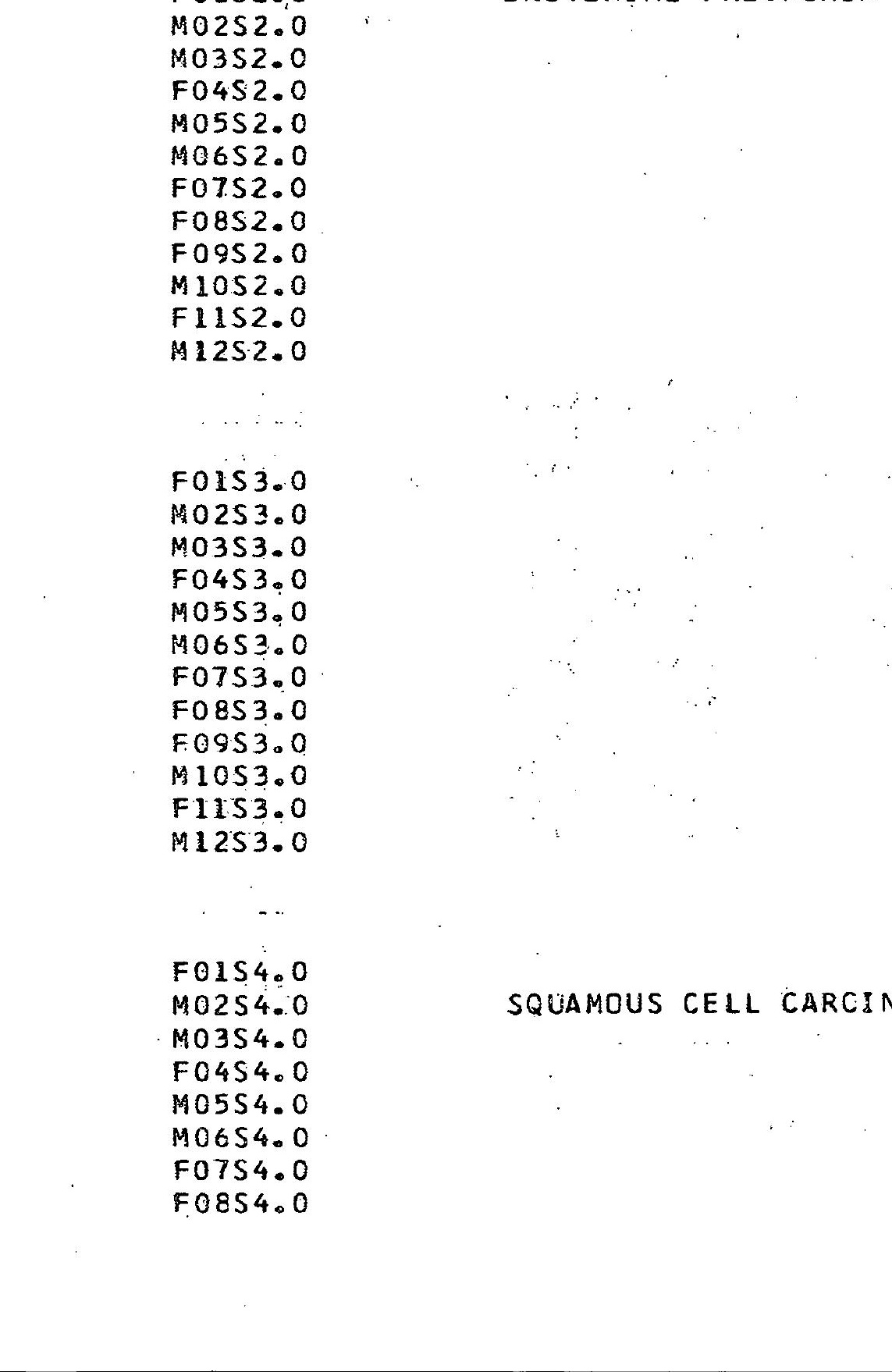




\begin{tabular}{|c|c|c|c|c|c|c|c|c|c|}
\hline $\begin{array}{l}\text { DOG } \\
\text { NUMB ER }\end{array}$ & $\begin{array}{l}\text { AT IN } \\
\text { AGE } \\
\text { IDAYSI }\end{array}$ & $\begin{array}{l}\text { JECTION } \\
\text { WE }[G H T \\
(K G)\end{array}$ & $\begin{array}{r}\text { INJECTED } \\
(\mu C / K G)\end{array}$ & $\begin{array}{c}D \\
\text { INJ } \\
D\end{array}$ & $\begin{array}{l}\text { ATE } \\
\text { JECT } \\
\text { MO }\end{array}$ & $\begin{array}{l}\text { TED } \\
\text { YR }\end{array}$ & $\begin{array}{l}\text { DAYS S } \\
\text { INJECT } \\
30 / 9 / 64\end{array}$ & $\begin{array}{l}\text { SINCE } \\
\text { TION } \\
\text { DEATH }\end{array}$ & $\begin{array}{l}\text { DOSE TO } \\
\text { SKELETO } \\
\text { IRADS }\end{array}$ \\
\hline \multirow[t]{2}{*}{$\begin{array}{l}\text { FOSS4.0 } \\
\text { M1OS4.0 } \\
\text { F11S4.0 } \\
\text { M12S4.0 }\end{array}$} & $\begin{array}{l}468 \\
517 \\
542 \\
605\end{array}$ & $\begin{array}{l}9.56 \\
8.20 \\
8.86 \\
10.9\end{array}$ & $\begin{array}{l}30.6 \\
31.3 \\
32.7 \\
32.3\end{array}$ & $\begin{array}{r}11 \\
29 \\
3 \\
6\end{array}$ & $\begin{array}{l}08 \\
09 \\
11 \\
01\end{array}$ & $\begin{array}{l}59 \\
59 \\
59 \\
60\end{array}$ & $\begin{array}{l}1877 \\
1828 \\
1793 \\
1729\end{array}$ & & \\
\hline & $\therefore$ & $\cdots=$ & $\ldots$ & $\therefore$ & .. & &.. & & \\
\hline $\begin{array}{l}F 01 S 5.0 \\
M 02 S 5.0 \\
M 02 S 5.0 A \\
M 03 S 5.0 \\
F 0455.0 \\
M 05 S 5.0 \\
M O 6 S 5.0 \\
M 0655.0 A \\
F O 7 S 5.0 \\
F O 8 S 5.0 \\
F 09 S 5.0 \\
M 1 O S 5.0 \\
F 11 S 5.0 \\
M 12 S 5.0\end{array}$ & $\begin{array}{l}434 \\
551 \\
545 \\
507 \\
528 \\
621 \\
504 \\
462 \\
478 \\
535 \\
459 \\
517 \\
542 \\
606\end{array}$ & $\begin{array}{l}9.3 .8 \\
12.2 \\
11.4 \\
10.3 \\
11.4 \\
.8 .53 \\
9.33 \\
11.2 \\
10.2 \\
11.2 \\
8.82 \\
8.55 \\
8.97 \\
12.5\end{array}$ & $\begin{array}{l}103 . \\
102 . \\
.96 .6 \\
102 . \\
105 . \\
.95 .2 \\
98.8 \\
94.2 \\
92.7 \\
90.5 \\
93.5 \\
95.9 \\
102 . \\
99.2\end{array}$ & $\begin{array}{l}18 \\
14 \\
27 \\
15 \\
15 \\
19 \\
-3 \\
7 \\
11 \\
7 \\
11 \\
29 \\
3 \\
6\end{array}$ & $\begin{aligned} 01 \\
02 \\
1 \\
10 \\
10 \\
11 \\
9 \\
01 \\
11 \\
01 \\
08 \\
09 \\
11 \\
01\end{aligned}$ & $\begin{array}{l}55 \\
56 \\
59 \\
57 \\
57 \\
57 \\
58 \\
59 \\
58 \\
59 \\
59 \\
59 \\
59 \\
60\end{array}$ & 1877 & $\begin{array}{r}960 \\
255 \\
1740 \\
2256 \\
1448 \\
1285 \\
1.35 \\
1021 \\
1129 \\
1469 \\
99 \\
1690 \\
1165\end{array}$ & $\begin{array}{r}7700 \\
3070 \\
11361 \\
15329 \\
.8758 \\
9381 \\
630 \\
11000 \\
10236 \\
10323 \\
.7335 \\
9385 \\
7874\end{array}$ \\
\hline
\end{tabular}


$D O G$ NUMBER

F0954.0 $M 1054.0$

F11.54.0

1254.0

F0155.0 M0255.0 $M 0255.0 A$ $M 0355.0$ F0 455.0 $M 0555.0$ $M 0655.0$ M.0655. OA F07S5.0 F08S5.0 F09S5.C M1OS5.0 F1155.0 M12S5.0

\section{COMMENTS ON DEAC DOES}

OSTEOSARCOMA
STRANGULATED INGUINAL HERNIA
OSTEOSARCONA
OSTEOSARCOMA
OSTEOSARCOMA

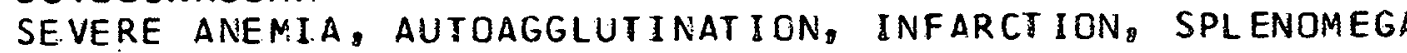
INTESTINAL HENORRHAGE OSTEOSARCOMA , INFARCTION + THROMBOCYTOPENIA STATUS EPILEPTICUS OSTEOSARCOMA

SE VERE ANEMIA + THROMBOCYTOPENIA HEMANG I OSARCOMA HEMANGI OSARCOMA 
TABLE II.TEST ANIMALS (SEPT:30. 1964)

$$
\text { A. RADIUM-226 }
$$

\begin{tabular}{|c|c|c|c|c|c|c|c|c|c|}
\hline $\begin{array}{l}\text { DOG } \\
\text { NUMBER }\end{array}$ & $\begin{array}{l}\text { AT II } \\
\text { AGE } \\
\text { IDAY }\end{array}$ & $\begin{array}{l}\text { JEC TI ON } \\
W E I G H T \\
(K G)\end{array}$ & $\begin{array}{c}\text { IN JEC TED } \\
(\mu C / K G)\end{array}$ & $\begin{array}{c}D \\
\text { IN } \\
D\end{array}$ & $\begin{array}{l}\text { JATE } \\
\text { JECT } \\
\text { MO }\end{array}$ & $\begin{array}{l}E \\
Y E D \\
Y R\end{array}$ & $\begin{array}{r}\text { DAYS } \\
\text { INJEC } \\
30 / 9.164\end{array}$ & $\begin{array}{l}\text { S INCE } \\
\text { I ION } \\
\text { DEATH }\end{array}$ & $\begin{array}{l}\text { DOSE TO } \\
\text { SK ELETON } \\
\text { (RADS.) }\end{array}$ \\
\hline 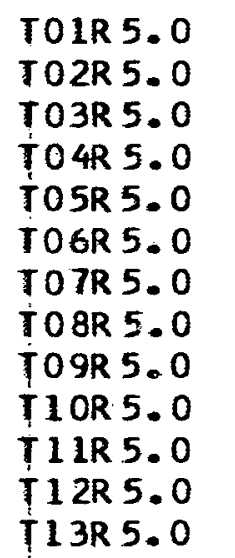 & $\begin{array}{r}995 \\
919 \\
1467 \\
459 \\
126 \\
126 \\
126 \\
290 \\
2276 \\
43 \\
43 \\
397 \\
397\end{array}$ & $\begin{array}{c}11.1 \\
8.40 \\
8.29 \\
10.0 \\
6.14 \\
6.14 \\
6.14 \\
5.52 \\
10.4 \\
1.02 \\
1.58 \\
12.3 \\
7.59\end{array}$ & $\begin{array}{c}10.3 \\
4.39 \\
4.76 \\
10.6 \\
11.7 \\
11.4 \\
11.8 \\
1.92 \\
1.94 \\
1.98 \\
1.91 \\
9.72 \\
9.76\end{array}$ & $\begin{array}{r}1 \\
12 \\
12 \\
6 \\
6 \\
6 \\
6 \\
10 \\
10 \\
10 \\
10 \\
9 \\
9\end{array}$ & $\begin{array}{l}12 \\
01 \\
01 \\
07 \\
10 \\
10 \\
10 \\
05 \\
05 \\
05 \\
05 \\
05 \\
05\end{array}$ & $\begin{array}{l}52 \\
.53 \\
53 \\
53 \\
53 \\
53 \\
53 \\
55 \\
55 \\
55 \\
55 \\
56 \\
56\end{array}$ & & $\begin{array}{r}1074 \\
1368 \\
428 \\
1 \\
1 \\
1 \\
1 \\
58 \\
58 \\
49 \\
49 \\
225 \\
188\end{array}$ & $\begin{array}{r}9490 \\
4733 \\
1320 \\
.53 \\
58 \\
57 \\
59 \\
501 \\
602 \\
300 \\
371 \\
2722 \\
2341\end{array}$ \\
\hline$\vdots$ & & $\ldots$ & - & -. & $\cdots$ & · & &..- & - \\
\hline $\begin{array}{l}14 R 4.0 \\
15 R 4.0\end{array}$ & $\begin{array}{l}674 \\
672\end{array}$ & $\begin{array}{l}8.12 \\
9.03\end{array}$ & $\begin{array}{l}3.17 \\
3.11\end{array}$ & $\begin{array}{l}11 \\
11\end{array}$ & $\begin{array}{l}07 \\
07\end{array}$ & $\begin{array}{l}56 \\
56\end{array}$ & & $\begin{array}{r}72 \\
2127\end{array}$ & $\begin{array}{r}390 \\
5123\end{array}$ \\
\hline : & .- & 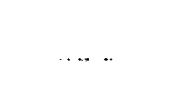 & & 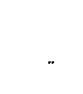 & & & & - & \\
\hline $\begin{array}{l}\text { T16R } 5.0 \\
17 R 5.0 \mathrm{H} \\
1 \mathrm{OR} 5 . \mathrm{OH} \\
19 \mathrm{R} 5 . \mathrm{OH} \\
2 \mathrm{OR} 5 . \mathrm{OH} \\
21 \mathrm{R} 5 . \mathrm{OH} \\
22 \mathrm{R} 5 . \mathrm{OH}\end{array}$ & $\begin{array}{r}604 \\
383 \\
383 \\
383 \\
383 \\
381 \\
381\end{array}$ & $\begin{array}{l}12.4 \\
12.2 \\
11.1 \\
10.67 \\
11.4: \\
11.8 \\
11.9\end{array}$ & 9.68 & $\begin{array}{l}11 \\
28 \\
28 \\
28 \\
28 \\
28 \\
28\end{array}$ & $\begin{array}{l}07 \\
10 \\
10 \\
10 \\
10 \\
10 \\
10\end{array}$ & $\begin{array}{l}57 \\
58 \\
58 \\
58 \\
58 \\
58 \\
58\end{array}$ & & $\begin{array}{r}12 \\
1147 \\
1226 \\
1219 \\
1330 \\
386 \\
587\end{array}$ & $\begin{array}{r}208 \\
13706 \\
12645 \\
11595 \\
12955 \\
3729 \\
5930\end{array}$ \\
\hline -. & & & & - & $\ldots$ & .. & & : & - \\
\hline $\begin{array}{l}23 \mathrm{R} 4 . \mathrm{OH} \\
24 \mathrm{R} 4 . \mathrm{OH} \\
\mathrm{T} 25 \mathrm{R} 4 . \mathrm{OH} \\
\mathrm{T} 26 \mathrm{R} 4 . \mathrm{OH} \\
2 \mathrm{R} 4 . \mathrm{OH}\end{array}$ & $\begin{array}{r}384 \\
: 384 \\
379 \\
379 \\
372\end{array}$ & $\begin{array}{l}9.50 \\
11.9 \\
11.3 \\
11.0 \\
11.5\end{array}$ & & $\begin{array}{l}25 \\
25 \\
25 \\
25 \\
25\end{array}$ & $\begin{array}{l}11 \\
11 \\
11 \\
11 \\
11\end{array}$ & $\begin{array}{l}58 \\
58 \\
58 \\
58 \\
58\end{array}$ & & $\begin{array}{l}1471 \\
1505 \\
1309 \\
1780 \\
1414\end{array}$ & $\begin{array}{l}4347 \\
5602 \\
4681 \\
4726 \\
3388\end{array}$ \\
\hline$\vdots$ & $\cdots$ & & & & . & & & & $\cdots$ \\
\hline T28R 3.OH & 372 & 11.7 & & 25 & 11 & 58 & & 387 & 359 \\
\hline$\ldots$. & $\cdots$ & & & & & & & $\cdots$ & \\
\hline T2 SR 5.0 & 474 & 13.5 & 10.4 & 3 & 03 & 59 & & 216 & 3434 \\
\hline
\end{tabular}


DOG

NUAB ER

TOLR 5.0

TO2R 5.0

TO3R 5.0

TO4R.5. 0

TOSR 5.0

TOSR 5.0

TOTR 5.0

TORR 5.0

TOQR 5.0

IIOR 5.0

IIIR 5.0

$T 12 R 5.0$

TI 3 R. 0

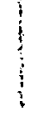

$14 R 4.0$

T15R 4.0

$116 R 5.0$

T17R 5. OH

$18 \mathrm{R} .5 . \mathrm{OH}$

TI $9 R 5 . \mathrm{OH}$

$20 R 5.0 H$

I $21 \mathrm{R} 5 . \mathrm{OH}$

T22R $5 . \mathrm{OH}$

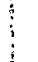

$23 R 4.0 \mathrm{OH}$

$\mathrm{T} 24 \mathrm{R} 4 . \mathrm{OH}$

T25R 4. OH

I 26R $4 . \mathrm{OH}$

T2 $7 \mathrm{R} 4 . \mathrm{OH}$

T28R 3. OH .

\section{COMMENJS ON DEAD DOGS}

SPECIAL STUDY OSTEOSARCOMA

SPECIAL STUDY

OSTEOSARCOMA + ULCERATIVE GINGIVITIS

OSTEOSARCOMA * ULCERATIVE GINGIVITIS

OSTEOSARCOMA - + ULCERATIVE GINGIVITIS

OSTEOSARCOMA + ULCERATIVE GINGIVITIS

NEPHRI IIIS

CRIPPLING FRACTURES

OSTE OSARCOMA

DSTEOSARCOMA

OSTEOSARCOMA

OSTEOSARCOMA

OSTEOSARCOMA

SPECIAL STUDY

$T 29 R 5.0$

NEPHRI TIS 


\begin{tabular}{|c|c|c|c|c|c|c|c|c|c|}
\hline $\begin{array}{l}\text { DOG } \\
\text { NUMB ER }\end{array}$ & $\begin{array}{l}\text { AT IN } \\
\text { AGE } \\
\text { (DAYS) }\end{array}$ & $\begin{array}{l}\text { JECTION } \\
\text { WEIGHT } \\
\text { (KG). }\end{array}$ & $\begin{array}{c}\text { I NJECTED } \\
(\mu \mathrm{C} / \mathrm{KG})\end{array}$ & $\begin{array}{c}D \\
\text { IN } \\
D\end{array}$ & $\begin{array}{l}\text { ATE } \\
\text { JECT } \\
\text { MO }\end{array}$ & $\begin{array}{l}\text { YED } \\
\text { YR }\end{array}$ & $\begin{array}{r}\text { DAYS S } \\
\text { INJECT } \\
30 / 9164\end{array}$ & $\begin{array}{l}\text { S INCE } \\
\text { T ION } \\
\text { DEATH }\end{array}$ & $\begin{array}{l}\text { DOSE TO } \\
\text { SK ELETON } \\
\text { (RADS) }\end{array}$ \\
\hline \multirow[t]{2}{*}{$\begin{array}{r}\text { TOR } 5.0 \\
\text { T3LR } 5.0\end{array}$} & $\begin{array}{l}47,4 \\
471\end{array}$ & $\begin{array}{l}11.5 \\
10.5\end{array}$ & $\begin{array}{l}10.4 \\
10.4\end{array}$ & $\begin{array}{l}3 \\
3\end{array}$ & $\begin{array}{l}03 \\
03\end{array}$ & $\begin{array}{l}59 \\
59\end{array}$ & & $\begin{array}{l}178 \\
303\end{array}$ & $\begin{array}{l}2879 \\
4788\end{array}$ \\
\hline & 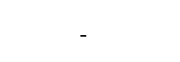 & - & $\cdots$ & & & & . & & \\
\hline $\begin{array}{l}32 R 3.0 \\
33 R 3.0 \\
34 R 3.0 \\
35 R 3.0 J\end{array}$ & $\begin{array}{l}47.1 \\
47.1 \\
470 \\
670\end{array}$ & $\begin{array}{c}11.4 \\
10.6 \\
15.7 \\
9.44\end{array}$ & $\begin{array}{r}1.13 \\
1.15 \\
1.12 \\
.951\end{array}$ & $\begin{array}{l}3 \\
3 \\
3 \\
5\end{array}$ & $\begin{array}{r}03 \\
3 \\
3 \\
5\end{array}$ & $\begin{array}{l}59 \\
59 \\
59 \\
59\end{array}$ & 2038 & $\begin{array}{r}1822 \\
1737 \\
8\end{array}$ & $\begin{array}{r}1924 \\
1606 \\
16\end{array}$ \\
\hline$\vdots \quad \ldots$ & . & $\cdots-$ & $\cdots-\cdots$ & . & & .. & & .. & : : \\
\hline $\begin{array}{r}36 R 4.0 \\
37 R 4.0 \\
38 R 4.0\end{array}$ & $\begin{array}{l}695 \\
695 \\
695\end{array}$ & $\begin{array}{l}10.2 \\
9.53 \\
10.1\end{array}$ & $\begin{array}{r}2.99 \\
3.00 \\
3.02\end{array}$ & $\begin{array}{l}22 \\
22 \\
22\end{array}$ & $\begin{array}{r}12 \\
12 \\
12\end{array}$ & $\begin{array}{l}60 \\
60 \\
60\end{array}$ & $\begin{array}{l}1378 \\
1378\end{array}$ & 1154 & 3654 \\
\hline . & & $\ldots$ & $\ldots$. & &... & . & $\ldots$ & & \\
\hline $\begin{array}{l}\text { T4OR } 1.0 \\
\text { T41R } 1.0\end{array}$ & $\begin{array}{l}899 \\
899\end{array}$ & $\begin{array}{l}13.0 \\
12.7\end{array}$ & $\begin{array}{l}.0483 \\
.0487\end{array}$ & $\begin{array}{l}3 \\
3\end{array}$ & $\begin{array}{l}4 \\
4\end{array}$ & $\begin{array}{r}62 \\
62\end{array}$ & & $\begin{array}{r}.7 \\
63\end{array}$ & $\begin{array}{l}1 \\
3\end{array}$ \\
\hline$\vdots$ & . & $\cdots$ & 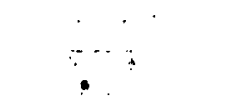 & $\because$ & & . & & & \\
\hline $\begin{array}{l}T 42 R 1.7 \\
T 43 R 1.7\end{array}$ & $\begin{array}{r}967 \\
: 963\end{array}$ & $\begin{array}{l}14.0 \\
13.2\end{array}$ & $\begin{array}{l}.146 \\
.145\end{array}$ & $\begin{array}{l}4 \\
4\end{array}$ & $\begin{array}{l}4 \\
4\end{array}$ & $\begin{array}{l}62 \\
62\end{array}$ & & $\begin{array}{r}7 \\
64\end{array}$ & $\begin{array}{r}2 \\
12\end{array}$ \\
\hline . & & $\because$ & & & &. & & & \\
\hline $\begin{array}{l}\text { T 44R 3.0 } \\
\text { T45R 3.0 } \\
\text { T46R 3.0 }\end{array}$ & $\begin{array}{l}938 \\
939 \\
810\end{array}$ & $\begin{array}{l}11.1 \\
13.6 \\
12.5\end{array}$ & $\begin{array}{r}.937 \\
.941 \\
.928\end{array}$ & $\begin{array}{l}4 \\
5 \\
5\end{array}$ & $\begin{array}{l}4 \\
4 \\
4\end{array}$ & $\begin{array}{l}62 \\
62 \\
62\end{array}$ & & $\begin{array}{r}68 \\
7 \\
69\end{array}$ & $\begin{array}{r}72 \\
14 \\
105\end{array}$ \\
\hline$\vdots$ & 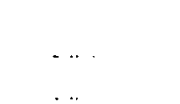 & $\cdots$ & $\cdots$ & & & & & & \\
\hline $\begin{array}{l}\text { T47R } 6.0 \\
\text { T } 48 R 6.0\end{array}$ & $\begin{array}{r}99 \\
2842\end{array}$ & $\begin{array}{l}5.27 \\
11.2\end{array}$ & $\begin{array}{l}29.4 \\
25: 1\end{array}$ & $\begin{array}{l}11 \\
27\end{array}$ & $\begin{array}{r}6 \\
12\end{array}$ & $\begin{array}{l}62 \\
62\end{array}$ & & $\begin{array}{r}4 \\
49\end{array}$ & $\begin{array}{r}440 \\
1633\end{array}$ \\
\hline & & . $\quad$. & $\ldots$ & & & & & & \\
\hline $\begin{array}{l}\text { T49R } 5.0 \\
\text { T } 5 \text { OR } 5.0 \\
\text { T } 5 \text { IR } 5.0 \\
\text { T } 5 \text { 2R } 5.0\end{array}$ & $\begin{array}{l}485 \\
485 \\
418 \\
418\end{array}$ & $\begin{array}{l}10.6 \\
13.7 \\
13.3 \\
10.7\end{array}$ & $\begin{array}{l}7.54 \\
7.46 \\
8.48 \\
8.57\end{array}$ & $\begin{array}{l}2 \\
2 \\
8 \\
8\end{array}$ & $\begin{array}{l}5 \\
5 \\
5 \\
5\end{array}$ & $\begin{array}{l}63 \\
63 \\
63 \\
63\end{array}$ & & $\begin{array}{r}5 \\
15 \\
92 \\
15\end{array}$ & $\begin{array}{r}133 \\
305 \\
1721 \\
286\end{array}$ \\
\hline
\end{tabular}


DOG

NUMBER

T3OR 5.0

T31R 5.0

T $32 R 3.0$

T $33 R 3.0$

T $34 R 3.0$

I $35 \mathrm{R} 3.0 \mathrm{~J}$

(2)

(0)

T 36R 4. 0

T 37R 4.0

T $38 R 4.0$

T4OR 1.0

T4IR1.0

$42 R 1.7$

I 4 R 1.7

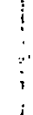

T4R 3.0

T 4 R 3.0

$46 R 3.0^{\circ}$

.

$47 R 6.0$

T48R 6. 0
T 49R 5.0

T SOR 5.0

TSIR 5.0

TS2R 5.0
COMMENTS ON DEAD DOGS

NE PHR ITIS

NEPHRI TIS

OSTEOSARCOMA, NEPHRITIS

OSTEOSARCOMA

SPECIAL STUDY

OS TEOSARCOMA

SPECIAL STUDY

SPECIAL STUDY

SPECIAL STUDY

SPECIAL STUDY

SPECIAL STUDY

SPECIAL STUDY

SPECIAL STUDY

SPECIAL STUDY

LEUKOPENIA. PNEUMONIA * SPECIAL MELANOMA STUDY

SPECIAL STUDY

SPECIAL STUDY

SPECIAL STUDY

SPECIAL STUDY 


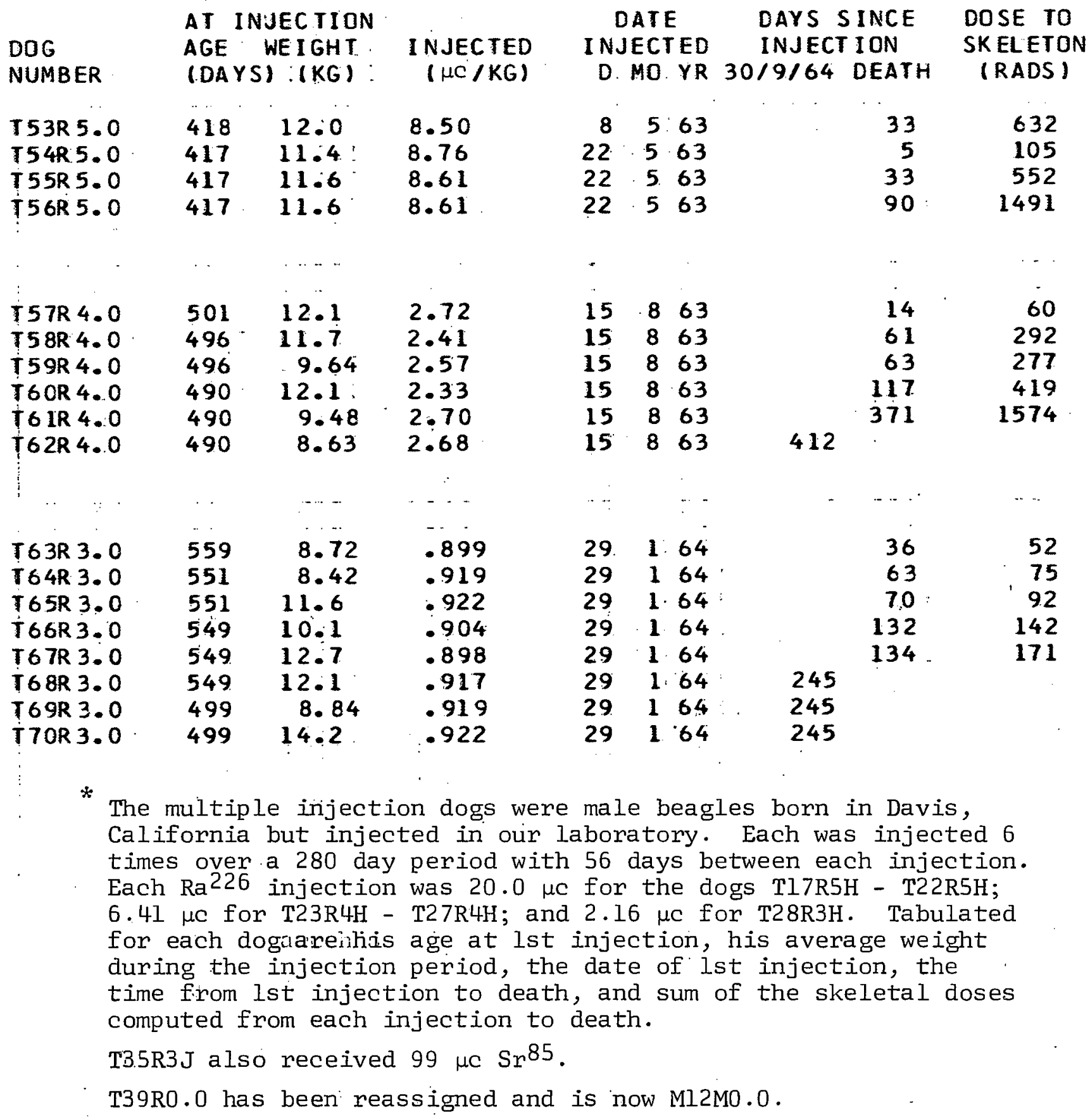


$D O G$

NUMBER

$153 R 5.0$

TS4R:5.0

$T 55 R 5.0$

T $6 R 5.0$

$\operatorname{TS} 7 \mathrm{R} 4.0$

$T 58 R 4.0$

T59R 4.0

T $60 R 4.0$

T6IR 4.0

$T 62 R 4.0$

$163 R 3.0$

T $64 R 3.0$

$T 65 R 3.0$

$T 66 R 3.0$

T $67 R 3.0$

T $68 \mathrm{R} 3.0$

T69R 3.0

T TOR 3.0
COMMENTS CN DEAD DOGS

\begin{abstract}
SPECIAL STUDY
SPECIAL STUOY

SPECIAL STUDY

SPECIAL STUDY
\end{abstract}

SPECIAL SJUDY

SPECIAL STUDY

SPECIAL STUDY

SPECIAL STUDY

SPECIAL STUDY

\section{SPECIAL STUDY \\ SPECIAL STUDY \\ SPECIAL STUDY \\ SPECIAL STUDY \\ SPECIAL STUDY}


B. PLUTONI UM-239

\begin{tabular}{|c|c|c|c|c|c|c|c|c|c|}
\hline $\begin{array}{l}\text { DOG } \\
\text { NUMBER }\end{array}$ & $\begin{array}{l}A T I \\
A G E \\
\text { IDAY }\end{array}$ & $\begin{array}{l}\text { JECTION } \\
\text { AE I GHT } \\
\text { (KG) }\end{array}$ & $\begin{array}{c}\text { INJEC TED } \\
(\mu \mathrm{K} / K G)\end{array}$ & IN & $\begin{array}{l}\text { ATE } \\
\text { JECT } \\
\text { MO }\end{array}$ & $\begin{array}{l}\text { ED } \\
\text { YR }\end{array}$ & $\begin{array}{l}\text { DAYS S } \\
\text { INJECT } \\
30 / 9 / 64\end{array}$ & $\begin{array}{l}\text { INCE } \\
\text { ION } \\
\text { DEATH }\end{array}$ & $\begin{array}{l}\text { DOSE TO } \\
\text { SKELETON } \\
\text { (RADS ) }\end{array}$ \\
\hline 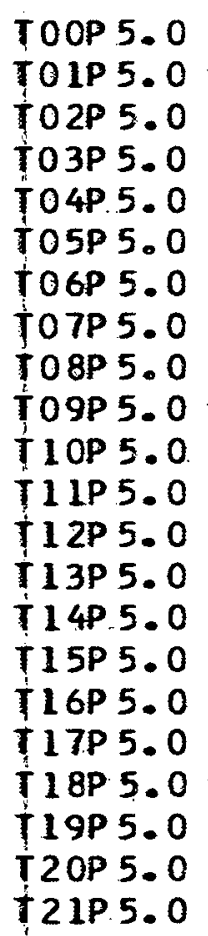 & $\begin{array}{l}647 \\
581 \\
914 \\
942 \\
1016 \\
474 \\
527 \\
475 \\
527 \\
551 \\
534 \\
516 \\
487 \\
587 \\
587 \\
737 \\
673 \\
739 \\
739 \\
688 \\
688 \\
688\end{array}$ & $\begin{array}{l}11.4 \\
12.7 \\
11.9 \\
9.65 \\
8.78 \\
10.4 . \\
6.16 \\
7.40 \\
8.32 \\
10 .-3 \\
11.9 \\
12.1 \\
9.23 \\
8.27 \\
9.38 \\
8.32 \\
10.7 \\
11.1 \\
8.16 \\
8.86 \\
13.0 \\
10.3\end{array}$ & $\begin{array}{l}3.05 \\
3.04 \\
6.85 \\
3.22 \\
3.02 \\
2.69 \\
2.73 \\
2.68 \\
2.67 \\
2.80 \\
2.7 .4 \\
2.76 \\
2.74 \\
3.16 \\
2.43 \\
2.79 \\
2.85 \\
3.01 \\
2.83 \\
2.91 \\
2.68 \\
2.72\end{array}$ & $\begin{array}{l}24 \\
13 \\
15 \\
13 \\
13 \\
14 \\
14 \\
14 \\
14 \\
22 \\
23 \\
22 \\
23 \\
24 \\
24 \\
15 \\
10 \\
12 \\
12 \\
15 \\
15 \\
15\end{array}$ & $\begin{array}{l}06 \\
10 \\
09 \\
10 \\
10 \\
12 \\
12 \\
12 \\
12 \\
11 \\
11 \\
11 \\
11 \\
04 \\
04 \\
10 \\
10 \\
02 \\
02 \\
12 \\
12 \\
12\end{array}$ & $\begin{array}{l}52 \\
52 \\
52 \\
52 \\
52 \\
54 \\
54 \\
54 \\
54 \\
55 \\
55 \\
55 \\
55 \\
56 \\
56 \\
56 \\
56 \\
57 \\
57 \\
60 \\
60 \\
60\end{array}$ & 1385 & $\begin{array}{r}1 \\
29 \\
44 \\
610 \\
365 \\
400 \\
406 \\
777 \\
863 \\
15 \\
15 \\
28 \\
28 \\
3 \\
7 \\
1 \\
92 \\
210 \\
217 \\
474 \\
939\end{array}$ & $\begin{array}{r}10 \\
160 \\
520 \\
3040 \\
1750 \\
1720 \\
1750 \\
3230 \\
3540 \\
80 \\
70 \\
140 \\
140 \\
20 \\
30 \\
10 \\
440 \\
990 \\
960 \\
1970 \\
3940\end{array}$ \\
\hline$:$ & $\begin{array}{l}: \\
\cdots \\
. .\end{array}$ & $\therefore$ & $\ldots$ & $\ldots$ & $\cdot-$ & $\cdots$ & & . & \\
\hline $\begin{array}{r}23 P 1.0 \\
24 P 1.0 \\
25 P 1.0 \\
26 P 1.0\end{array}$ & $\begin{array}{r}1485 \\
559 \\
559 \\
556\end{array}$ & $\begin{array}{l}13.1 \\
13.1 \\
13.8 \\
12.0\end{array}$ & $\begin{array}{l}.0172 \\
.0172 \\
.0167 \\
.0160\end{array}$ & $\begin{array}{l}28 \\
28 \\
28 \\
28\end{array}$ & $\begin{array}{l}7 \\
7 \\
7 \\
7\end{array}$ & $\begin{array}{l}61 \\
61 \\
61 \\
61\end{array}$ & & $\begin{array}{r}96 \\
97 \\
467 \\
647\end{array}$ & $\begin{array}{l}10 \\
20\end{array}$ \\
\hline$!$ &. & & $\cdots \cdots$ & $\cdots$ & 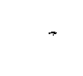 & & & & \\
\hline T27P 3.0 & 556 & $11: 5$ & .332 & 28 & 7 & 61 & & $\begin{array}{c}755 \\
:\end{array}$ & 390 \\
\hline T $28 \mathrm{P} 1.0$ & 552 & 10.5 & .0150 & 9 & 8 & 61 & & 559 & 10 \\
\hline T 29P. 3.0 & 552 & 12.1 & .296 & 9 & 8 & 61 & . & 560 & 260 \\
\hline T3OP 1.0 & 548 & 12.4 & .0148 & 9 & 8 & 61 & & 35 & \\
\hline
\end{tabular}




$$
-47 \div
$$

DOG

NUMBER -

TOOP 5.0

TO1P 5.0

r02P 5.0

T03P 5.0

to $4 P 5.0$

TO5P 5.0

TO6P 5.0

TO7P 5:0

TO 8P.5.0

TO SP 5.0

T1OP 5.0

$11 P 5.0$

T12P 5.0

$13 P 5.0$

$114 P 5.0$

T1.5P 5.0

T $16 P 5.0$

117.05 .0

T 1 \&P 5.0

$19 P 5.0$

T20P 5.0

$21 \mathrm{P} 5.0$

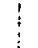

T23P $1: 0$

T24P.1.0

T25P 1.0

T26P1.0

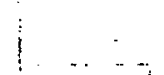

2783.0

$\vdots$

T $28 \mathrm{P} .1 .0$

T29P 3.0

T3OP 1.0
COMMENTS ON DEAD DOGS

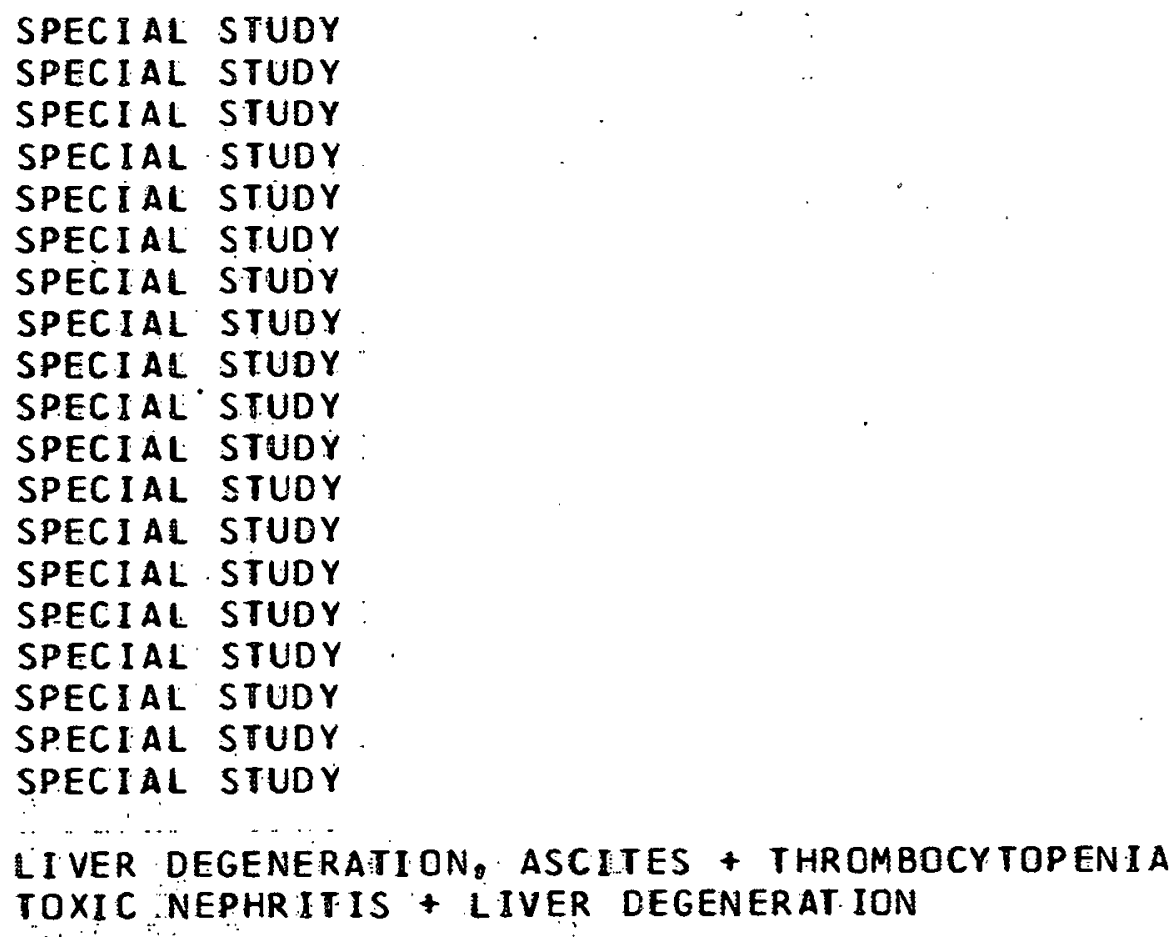

I I VER DEGENERATION: ASCITES + THROMBOCYTOPENIA TOXIC NEPHRITIS + LIVER DEGENERATION

SPECIAL STUDY

SPECIAL STUDY

SPECIAL STUDY

SPECIAL STUDY.

SPECIAL STUDY

SPECIAL STUDY

SPECIAL STUDY

SPECIAL STUDY 


\begin{tabular}{|c|c|c|c|c|c|c|c|c|}
\hline $\begin{array}{l}\text { DOG } \\
\text { NUMBER }\end{array}$ & $\begin{array}{l}\text { A T } 1 \\
\text { AGE } \\
\text { (DA) }\end{array}$ & $\begin{array}{l}\text { JEC TION } \\
\text { WEIGHT: } \\
\text { J (KG) }\end{array}$ & $\begin{array}{r}\text { INJEC TED } \\
(\mu \mathrm{K} / \mathrm{KG})\end{array}$ & \multicolumn{2}{|c|}{ DATE } & $\begin{array}{l}E D \\
Y R\end{array}$ & $\begin{array}{c}\text { DAYS SINCE } \\
\text { INJECTION } \\
30 / 9 / 64 \text { DEATH }\end{array}$ & \multirow{2}{*}{$\begin{array}{c}\text { DOSE TO } \\
\text { SKELETON } \\
\text { (RADS) } \\
20\end{array}$} \\
\hline T $31 \mathrm{IP} 3.0$ & 519 & 13.0 & .305 & 9 & 8 & 61 & 40 & \\
\hline & & & $\cdots$ & . & & . & - & \\
\hline $\begin{array}{l}\text { T32P } 1.0 \\
T 33 P 1.0 \\
T 34 P 1.0\end{array}$ & $\begin{array}{l}520 \\
550 \\
550\end{array}$ & $\begin{array}{l}8.47 \\
10.7 \\
9.68\end{array}$ & $\begin{array}{l}.0162 \\
.0153 \\
.0154\end{array}$ & $\begin{array}{r}9 \\
15 \\
15\end{array}$ & $\begin{array}{l}8 \\
9 \\
9\end{array}$ & $\begin{array}{l}61 \\
61 \\
61\end{array}$ & $\begin{array}{l}274 \\
375 \\
746\end{array}$ & $\begin{array}{l}10 \\
10 \\
20\end{array}$ \\
\hline : & & $\ldots, \ldots$ & $\ldots$ & . & & . & . & \\
\hline $135 P 3.0$ & 550 & 11.9 & .303 & 15 & 9 & 61 & 362 & 180 \\
\hline$!$ & & & & & & & & \\
\hline $\begin{array}{l}\text { T36P } 1.0 \\
\text { T37P } 1.0\end{array}$ & $\begin{array}{l}544 \\
542\end{array}$ & $\begin{array}{r}10.4 \\
8.59\end{array}$ & $\begin{array}{l}.0158 \\
.0148\end{array}$ & $\begin{array}{l}15 \\
15\end{array}$ & 9 & $\begin{array}{l}61 \\
61\end{array}$ & $\begin{array}{r}5 \\
186\end{array}$ & \\
\hline & & & $\cdots \therefore:$ & $\ldots .$. & & - & . & : \\
\hline T $38 \mathrm{P} 3.0$ & 489 & 7.96 & $.304 ?$ & 15 & 9 & 61 & 187 & 90 \\
\hline $\begin{array}{l}\text { T39P } 1.0 \\
\text { T4OP } 1.0\end{array}$ & $\begin{array}{l}1534 \\
1534\end{array}$ & $\begin{array}{c}10.7 \\
9.92\end{array}$ & $\begin{array}{l}.0151 \\
.0177\end{array}$ & $\begin{array}{l}15 \\
15\end{array}$ & $\begin{array}{l}9 \\
9\end{array}$ & $\begin{array}{l}61 \\
61 .\end{array}$ & $\begin{array}{l}376 \\
769\end{array}$ & $\begin{array}{l}10 \\
20\end{array}$ \\
\hline
\end{tabular}


DOG

NUMBER -

$31 P 3.0$

T $32 \mathrm{P} 1.0$

T33P 1.0

T34P.1.0

$135 P 3.0$

T $36 P 1.0$

T $37 P 1.0$

T $38 \mathrm{P} 3.0$

T39P 1.0

T $40 P 1.0$

\section{COMMENTS ON DEAD DOGS}

SPECIAL STUDY

SPECIAL STUDY

SPECIAL STUDY

SPECIAL STUDY

SPECIAL STUDY

SPECIAL STUDY

SPECIAL STUDY

SPECIAL STUDY

SPECIAL STUDY

SPECIAL STUDY 


\section{RADIUM-228 (MESOTHORIUM) *}

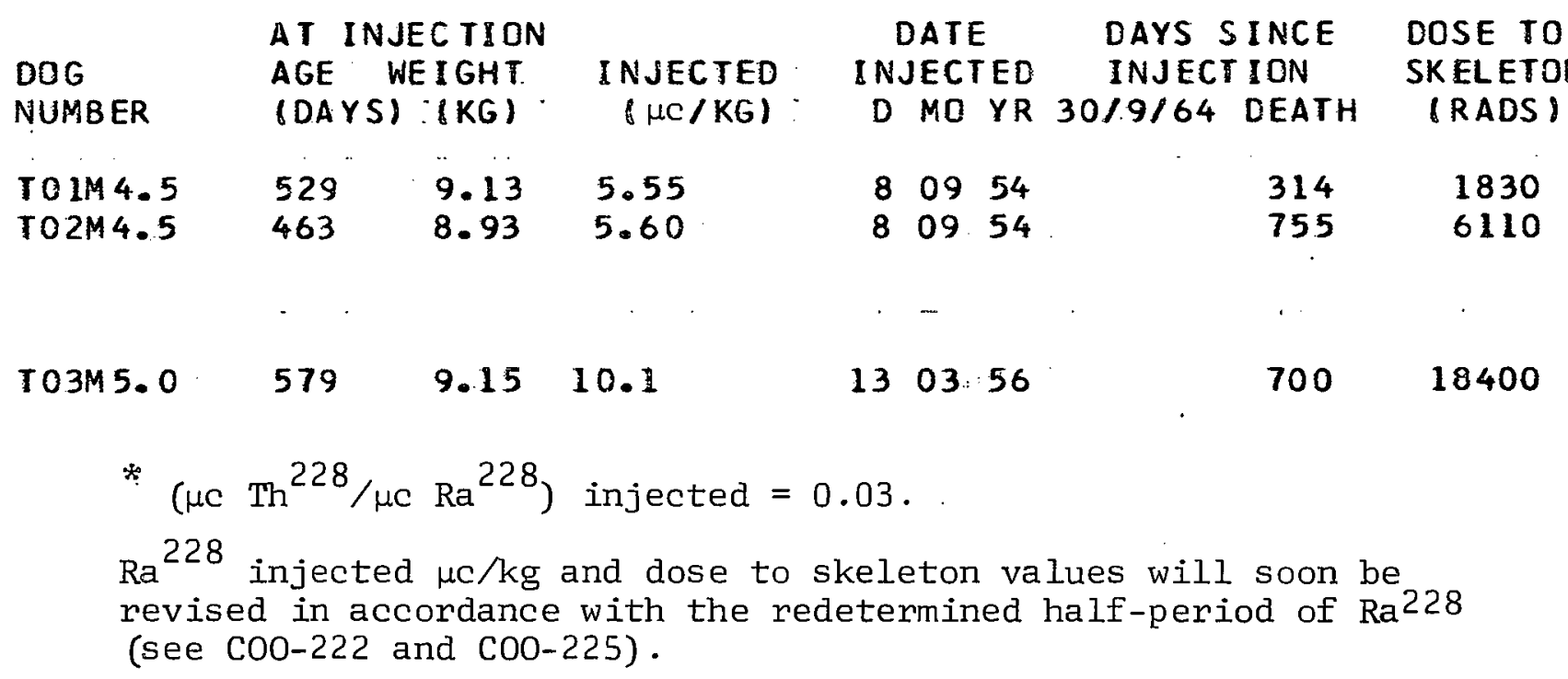


DOG

NUMBER

TO1M4: 5

TO2M 4..5

TO 3M 5. 0
COMMENTS: ON DEAD DOES

CANINE DISTEMPER

SPECIAL STUDY

ULCERATIVE“GINGIVITIS; SEVERE ANEMIA \&. CR IPPL ING FRACTUR 
D. THORIUM-228 (RADIOTHORIUM) *

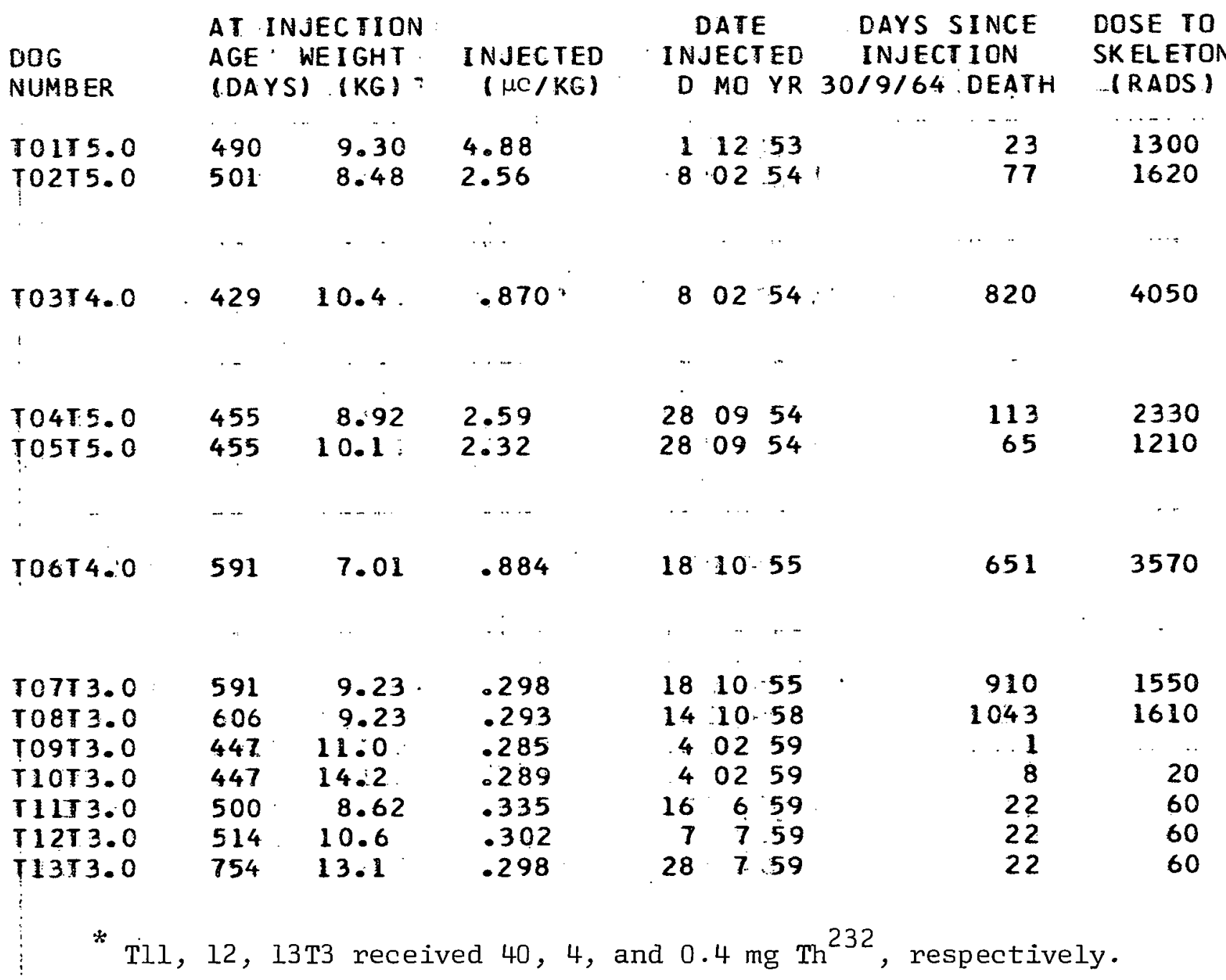


DO $G$

NUMBER

T. 015.0

$102 T 5.0$

$103 T_{4.0}$

10475.0

$105 T 5.0$

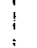

T06T4.0

10753.0

T०8 3.0

10913.0

T10T3.0

$111 \pi 3.0$

T12T3.0

$13 \mathrm{~T} 3.0$

\section{COMMENTS ON DEAD DOGS}

DIED, SPECIAL STUDY

SPECIAL STUDY

CRIPPLING FRACTURES + NEPHRITIS

THROMBOCYTOPENIA + PURPURA

NEPHRITIS: THROMBCCYTOPENIA \& PURPURA

CRIPPLING FRACTURES

SPECIAL STUDY

OSTEOSARCOMA

SPEC IAL STUDY

SPECIAL STUDY

SPECIAL STUDY

SPECIAL STUDY

SPECIAL STUDY 
E. STRONTIUM-90*

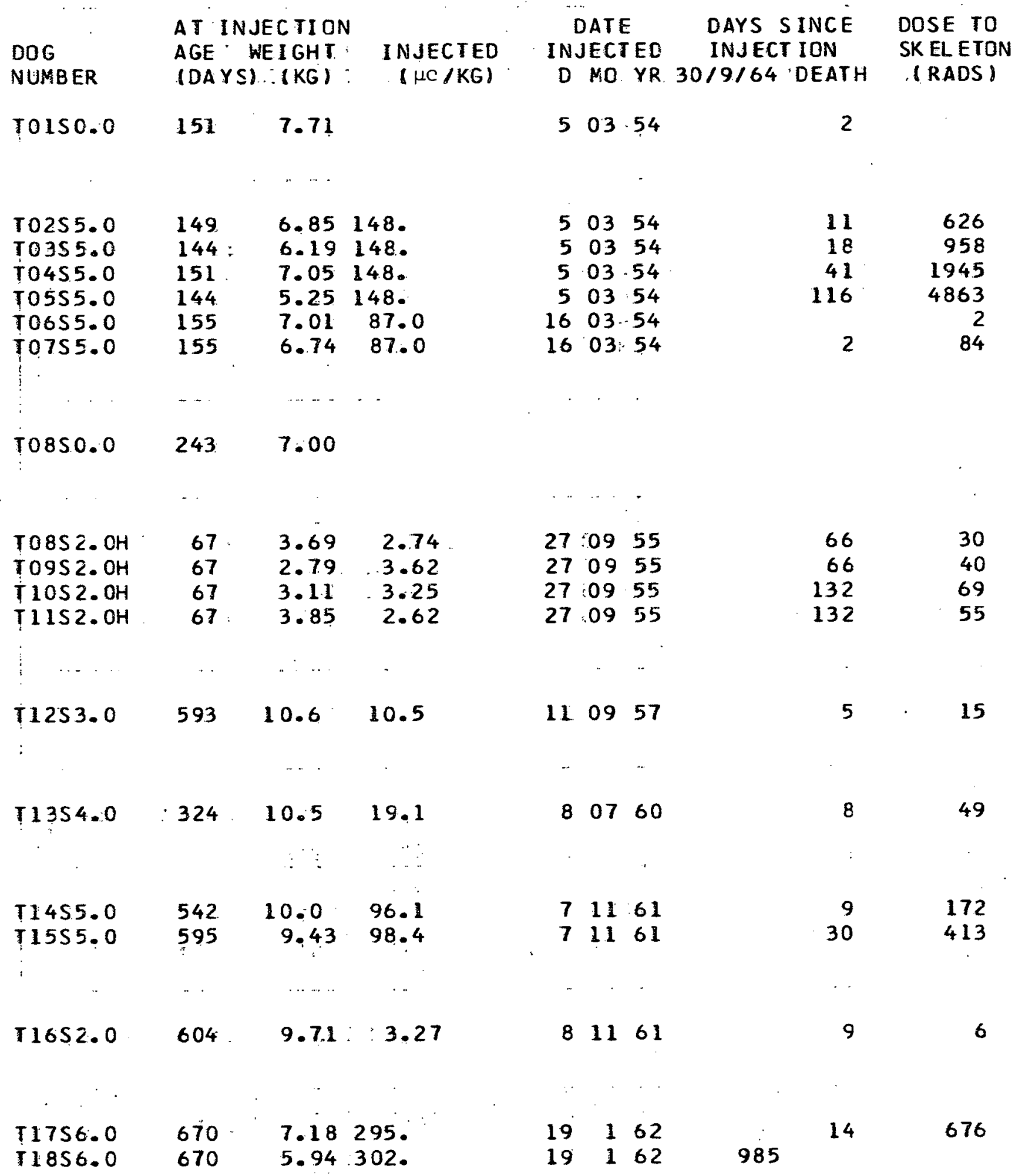


DOG

NUMBER

10150.0

10255.0

10355.0

T04S5.0

10555.0

10655.0

10755.0

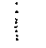

T085 .0

TOES $2.0 \mathrm{OH}$

TO9S2. $\mathrm{CH}$

$110 \mathrm{~S} 2 . \mathrm{OH}$

$11 \mathrm{~S} 2 . \mathrm{OH}$

125300

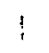

1354.0

T14S.5. 0

1555.0

1652.0

1756.0

1856.0

\section{COMMENTS ON DEAD DOGS}

SPECIAL STUDY

SPECIAL STUDY

SPECIAL STUDY

SPECIAL STUDY

SPECIAL STUDY

SPECIAL STUDY

SPECIAL STUDY

SPECIAL STUDY

SPECIAL STUDY

SPECIAL STUDY

SPECIAL STUDY

SPECIAL STUDY

BREMSSTRAHLUNG PHANTOM

BREMSSTRAHLUNG PHANTCM SAM MCGEE

SPECIAL STUDY

SPECIAL STUDY.

SPECIAL STUDY

LEUKOPENIA : THROMBOCYTOPENIA + PURPURA 


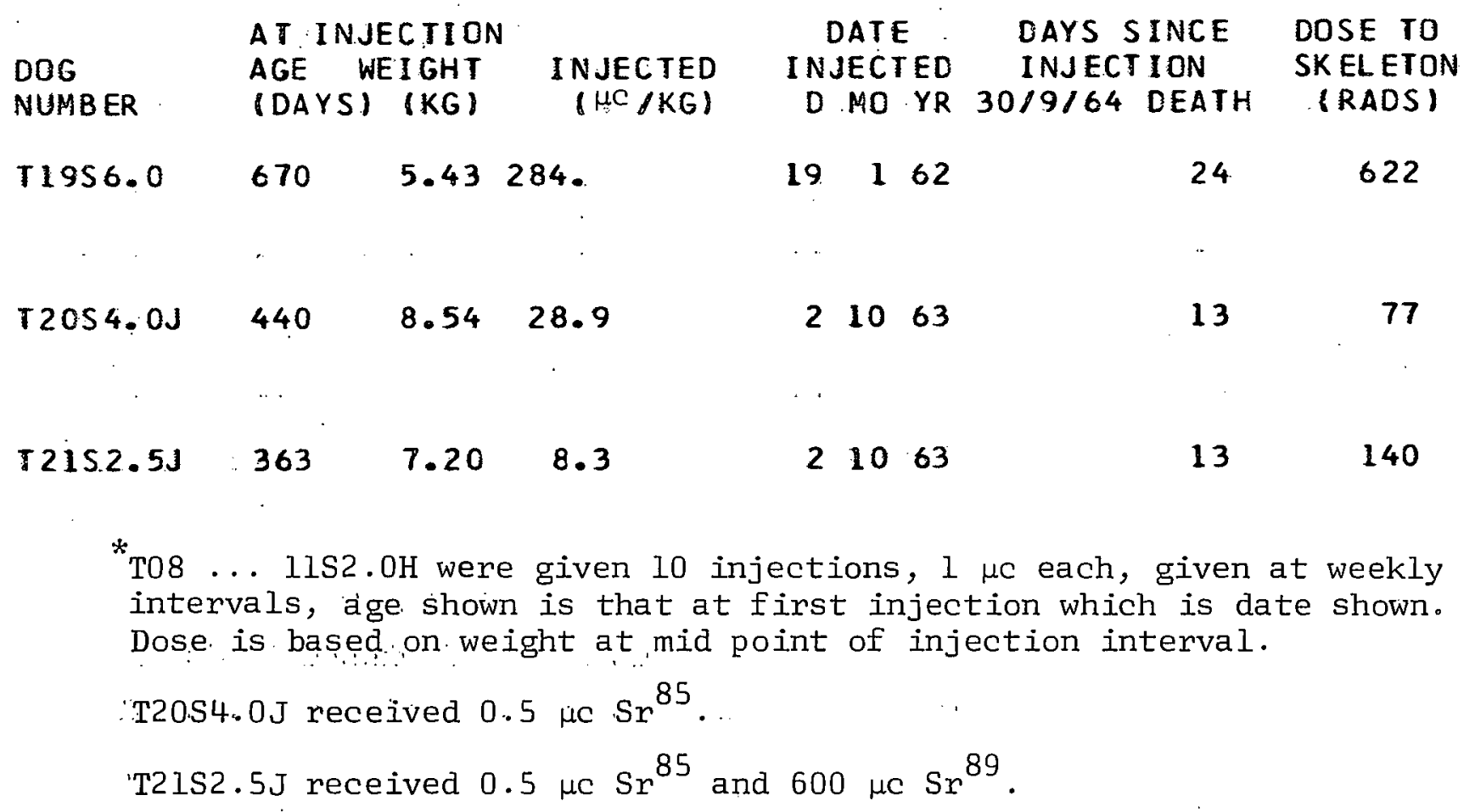


$\therefore \quad-57 \div$

(6)

(10).

DOG

NUMBER :

T19S6:0

$\mathrm{T} 2054.0 \mathrm{~J}$

$12152.5 \mathrm{~J}$

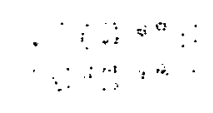

COMMENTS ON DEAD DOGS

LEUKOPENIA \& THROMBOCYTOPENIA * PURPURA

SPECIAL STUDY:

SPECIAL STUDY

(a)

E)

(ख) 
F. RADIUM-224

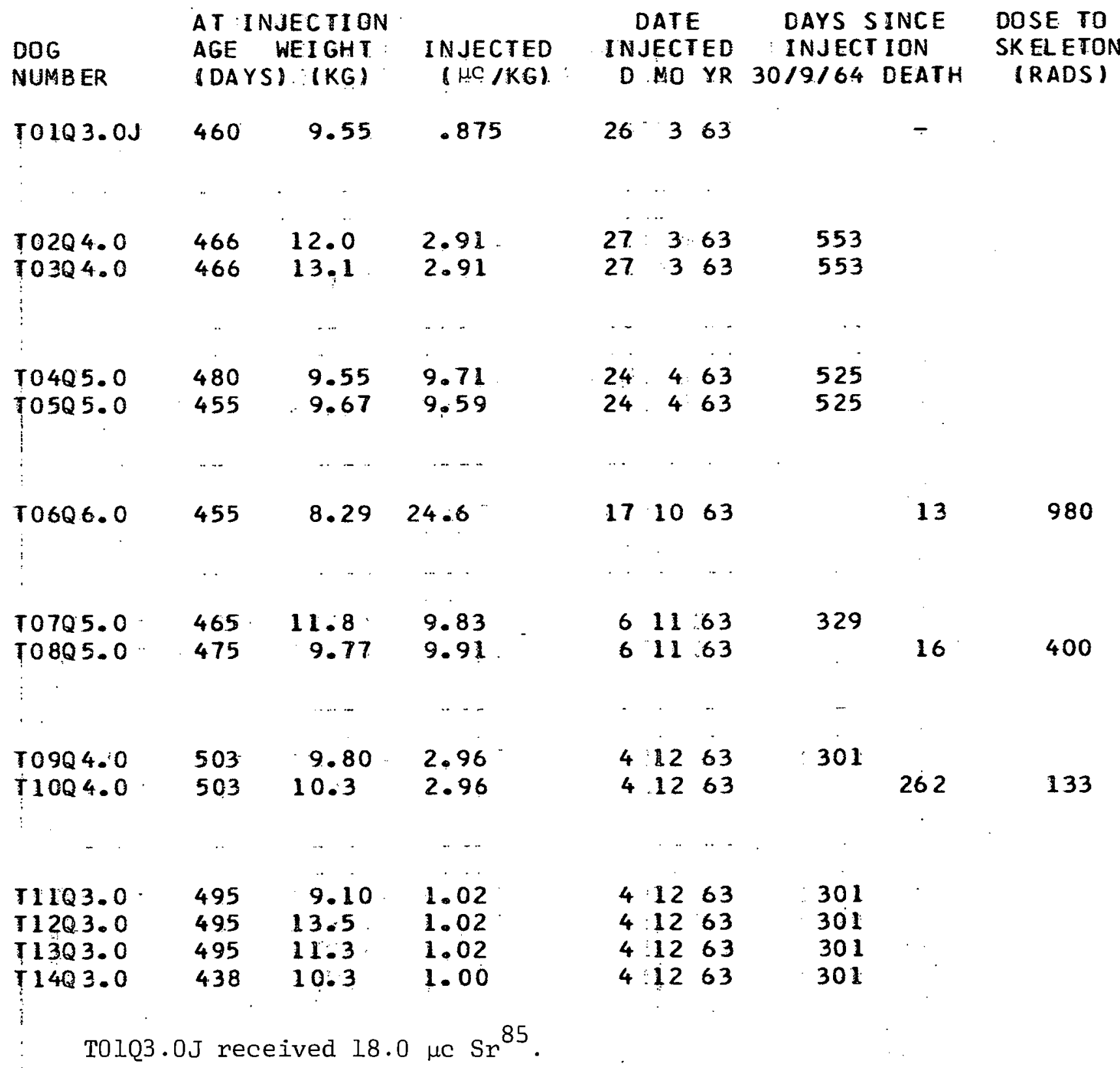




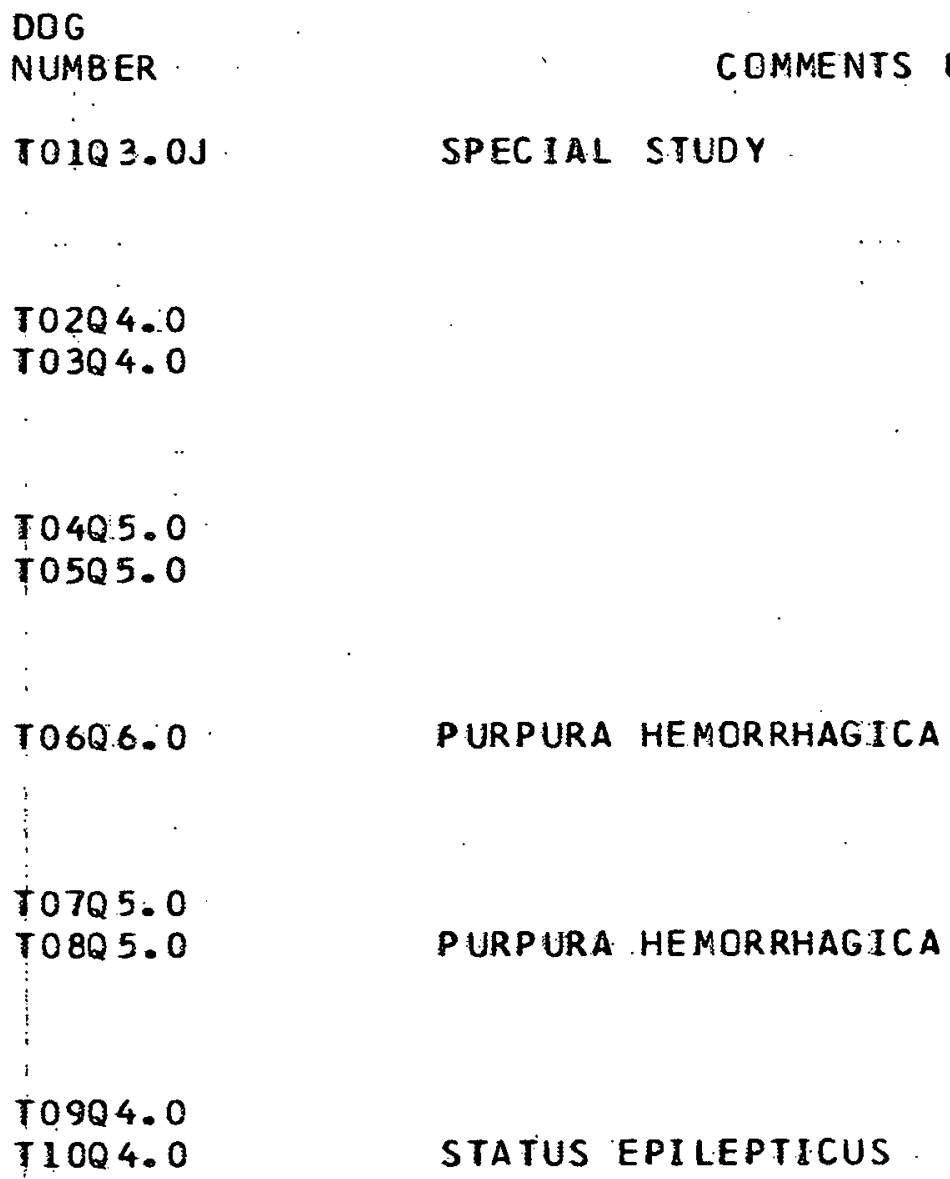

STATUS EPILEPTICUS

P URP URA . HE MORRHAGICA

1103.0

1203.0

T 1303.0

$114 Q 3.0$ 


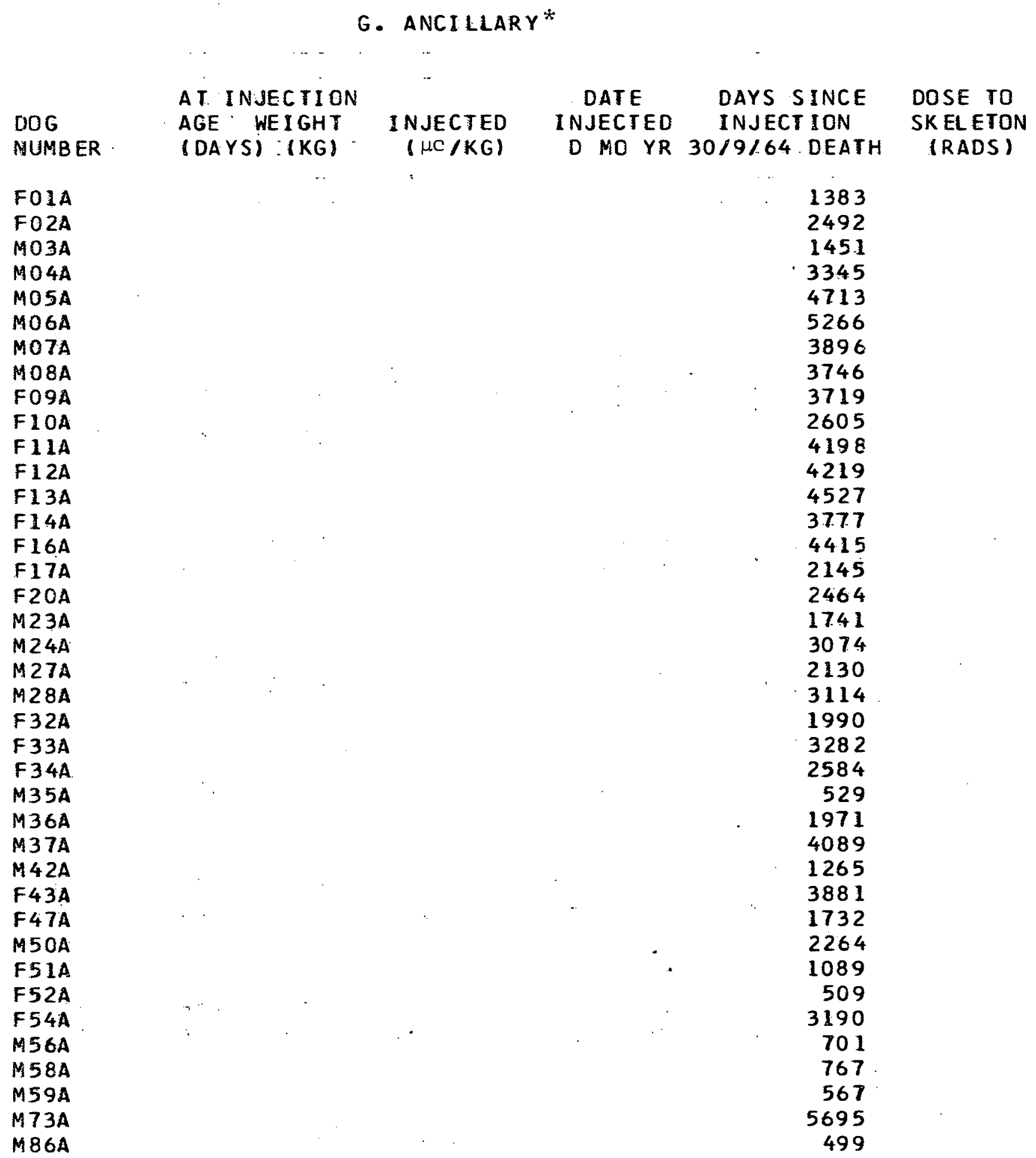

* Time interval shown here is the animal's age. 
DOG

NUMB ER

FO1A

F02A

MO3A

M04A

MO5A

MO6A

MOTA

MO8A

F0.9A

F10A

FIIA

F12A

F13A

F14A

F16A

F17.A

F20A

M23A

$M 24 A$

M27.A

M28A

F32A

F $33 \mathrm{~A}$

F 34A

M35A

M36A

M37A

$M 42 A$

F43A

F47A

MSOA

F51A

F $52 \mathrm{~A}$

F54A

M56A

M58A

M59A

M73A

M 86A

SPECIAL STUDY

SPECIAL STUDY

SPECIAL STUDY

NOT.DETERMINED

BRAIN HEMORRHAGE

LYMPHOSARCOMA

PRDGRESSIVE :PARALYSIS, CAUSE UNKNOWN

VAGINAL FIBROMA:

SPECI.AL STUDY

MAMMARY CARCINOMA

SEVERE OSTEQARTHRITIS

SPECIAL STUDY

OBTURATING EMBOLISM OF PORTAL VEIN

SPECIAL STUDY

TRAUMA

SPECIAL STUDY

OBTURATING PULMONARY EMBOLISM

SPECIAL STUDY

SPECIAL. STUDY

SPECIAL STUDY

LYMPHOSARCOMA

OB TURA IING: PULMONARY EMBOLISM

SPECIAL STUDY

SPECIAL STUDY

SPECIAL STUUY

SPECIAL STUDY

STATUS: EPILEPTICUS

SPECIAL. STUDY

SPECIAL STUDY

SPECIAL. STUDY

SPECIAL STUDY

SPECIAL STUDY

SPECIAL STUDY

VOLVULUS + PERITONITIS

SPECIAL STUDY

SPECIAL STUDY

DEGENERATION OF ADRENAL GLAND + DIABETES MELLITUS SPECIAL STUDY 


$\begin{array}{llcccc} & \text { AT INJECTION } & \text { DATE } & \text { DAYS SINCE DOSE TO } \\ \text { DOG } & \text { AGE WEIGHT } & \text { INJECTED } & \text { INJECTED } & \text { INJECTION } & \text { SKELETON } \\ \text { NUMBER } & \text { (DAYS):(KG) } & (1 K G) & \text { D MOYR } & \text { DEATH } & \text { (RADS) }\end{array}$


REPEATED USE OF ANESTHESIA IN AN EXPERIMENTAL DOG COLONY
G. N. Taylor
C. E. Rehfeld,
$\mp$

From

Abstract: Extensive use of thiopental sodium anesthesia over a ten year period, primarily for restraint purposes, has proven to be a safe procedure in beagles. Two anesthetic deaths resulted from 8,083 anesthetics. given to 702 dogs at a mean interval of approximately 100 days. Residual effects were not detected. The age classes ranged from 3 months to 14 years and included various degrees of health.

Some of the techniques used in evaluating the beagles at this laboratory are best performed on the anesthetized animal. The quality of radiographs is markedly improved, and the hazard to the $x$-ray technician is eliminated (Fig. 1). The precision of whole-body counting is significantly enhanced. Some of our whole-body counters could not be used on the unanesthetized animal (Figs. $2 \& 3$ ). Other procedures such as dental work, etc., are facilitated.

This laboratory has used thiopental sodium "anesthesia in conjunction with these techniques for over 10 years and it is the purpose of this study to indicate the degree of safety that we have experienced with this drug.

\section{METHODS}

Approximately 95\% of the cases listed in Table I were anesthetized to facilitate whole-body counting or radiography. In most instances thiopental sodium without any preanesthetic medication was the anesthetic

\footnotetext{
*Pentothal sodium, Abbott Laboratories, North Chicago, Illinois.

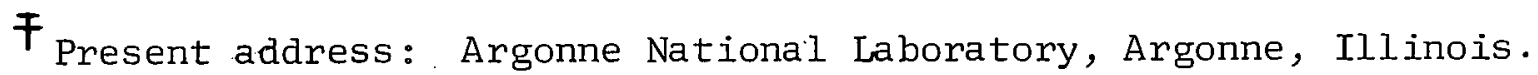


used. Ether* or methoxyflurane ${ }^{* *}$ was used in the relatively small number of exceptions--usually for surgical procedures. However, thiopental sodium was also used in these cases to facilitate induction and intubation. Morphine and/or atropine premedication was generally used in conjunction with the volatile anesthetics.

The desired depth of thiopental sodium anesthesia was maintained by taping the syringe, with the needle inserted into the cephalic vein, to the foreleg and injecting additional amounts as needed (Fig. 1). The duration varied from a few minutes to several hours--averaging approximately 30 to 45 minutes (not including recovery phase). In most instances, especially when used for restraint purposes, the depth was light and did not exceed the top of plane one of the surgical stage as adjudged by skeletal muscle tone (muscles of mastication), pupil size; palpebral and pedal reflexes $(1 ;, 2)$.

During the recovery period the dogs were caged in a controlledtemperature room and observed at 10 minute intervals until such time as they were able to raise their heads. Following this stage of recovery, which averaged approximately 80 minutes, the frequent observations were stopped, but cage confinement was continued until completely coordinate. The dog was, then returned to its regular run or a separate hospital run if surgery had been performed.

\section{RESULTS}

Table 1 summarizes 8,083 anesthetics administered from 1951 through 1964. These cases represent beagles of varying degrees of health ranging

*Ether, Mallinckrodt Chemical, Works, St. Louis, Missouri. ** Penthrane, Abbott Laboratories, North Chicago, Illinois. 
from excellent to very poor. Many exhibited radiation induced blood dyscrasias, skeletal fractures, osteosarcomas, liver lesions, kidney disease, and miscellaneous conditions. In some cases a combination of some of these conditions existed. The age groups extend from 3 months to 14 years, and seṇile changes were obvious in a significant per cent of the older animals.

Anesthesia was not used in certain instances where the risk appeared great.

The number of anesthetics given to a single dog ranged from 4 to 89. Two animals, one male and one female, were anesthetized 89 times at one month intervals. This sequence of anesthesia was begun at 6 months of age. Both of these dogs, now 8-1/2 years old, are presently asymptomatic.

In spite of the varied age and health status and the relatively large number of anesthetics given, only 2 anesthetic accident deaths resulted from the administration of 8,083 anesthetics. Also, frequent clinical, hematological and blood chemistry examinations have not indicated any residual affects resultant to thiopental sodium anesthesia even in the two dogs anesthetized 89 times $(3,4) *$. This relatively low mortality is attributed to the light, but nevertheless adequate depth of anesthesia generally used, to the very low toxicity of thiopental sodium, and to strict adherance to acceptable techniqües.

\section{DISCUSSION}

The use of anesthesia in experimental animals is certainly upen to criticism, and it has been used with some reluctance at this laboratory.

\footnotetext{
* This observed safety is in agreement with many other studies (5-9).
} 
However, in our program, elimination of this form of restraint would greatly curtail or preclude some important aspects of our study. The matter then becomes one of deciding if the biological price, if any, that we might be paying for such usage justifies the benefits.

\section{TABLE 1}

Summary of Anesthetics Used in Beagle Colony, University of Utah Radiobiology Laboratory, 1951 Through 1964.

Thiopental Sodium was Used in Most Instances.

\begin{tabular}{|c|c|c|c|c|c|}
\hline Nuclide & $\begin{array}{l}\text { Total \# } \\
\text { Anesthetics } \\
\text { Given* }\end{array}$ & $\begin{array}{l}\text { Total \# } \\
\text { Dogs } \\
\text { Anesthetized }\end{array}$ & $\begin{array}{l}\text { Average \# } \\
\text { Anesthetics } \\
\text { Per Dog }\end{array}$ & $\begin{array}{l}\text { Maximum \# } \\
\text { Anesthetics } \\
\text { For Any } \\
\text { Single Dog }\end{array}$ & $\begin{array}{l}\text { Mean } \\
\text { Interval } \\
\text { Between } \\
\text { Anesthetics } \\
\text { (Days) } \\
\end{array}$ \\
\hline Plutonium & 1506 & 139 & 11 & 32 & 151 \\
\hline Radium $^{226}$ & 2280 & 153 & 15 & 41 & 113 \\
\hline Mesothorium & 1047 & 92 & 11 & 33 & 129 \\
\hline Strontium & 1401 & 99 & 14 & 37 & 131 \\
\hline Radiothorium & 1082 & 107 & 10 & 32 & 117 \\
\hline Radium 224 & 31 & 13 & 2 & 4 & 146 \\
\hline X-Ray Controls & 318 & 6 & 53 & 89 & 33 \\
\hline Aging Controls & 388 & 80 & 5 & 13 & 361 \\
\hline Ünassigned & 20 & 6 & 3 & 8 & 44 \\
\hline Others & 10 & 7 & 1 & 4 & 19 \\
\hline Total & 8083 & 702 & 12 & & \\
\hline
\end{tabular}


Our experience, extending over the past ten years, indicates the use of anesthesia to the extent covered in this paper is justified and is compatible with the objectives of our experiment. We are not aware of other studies that have demonstrated irreversible changes within the limits of our usage.

The authors wish to thank L. Shabestari and L. Brewster for their technical assistance.

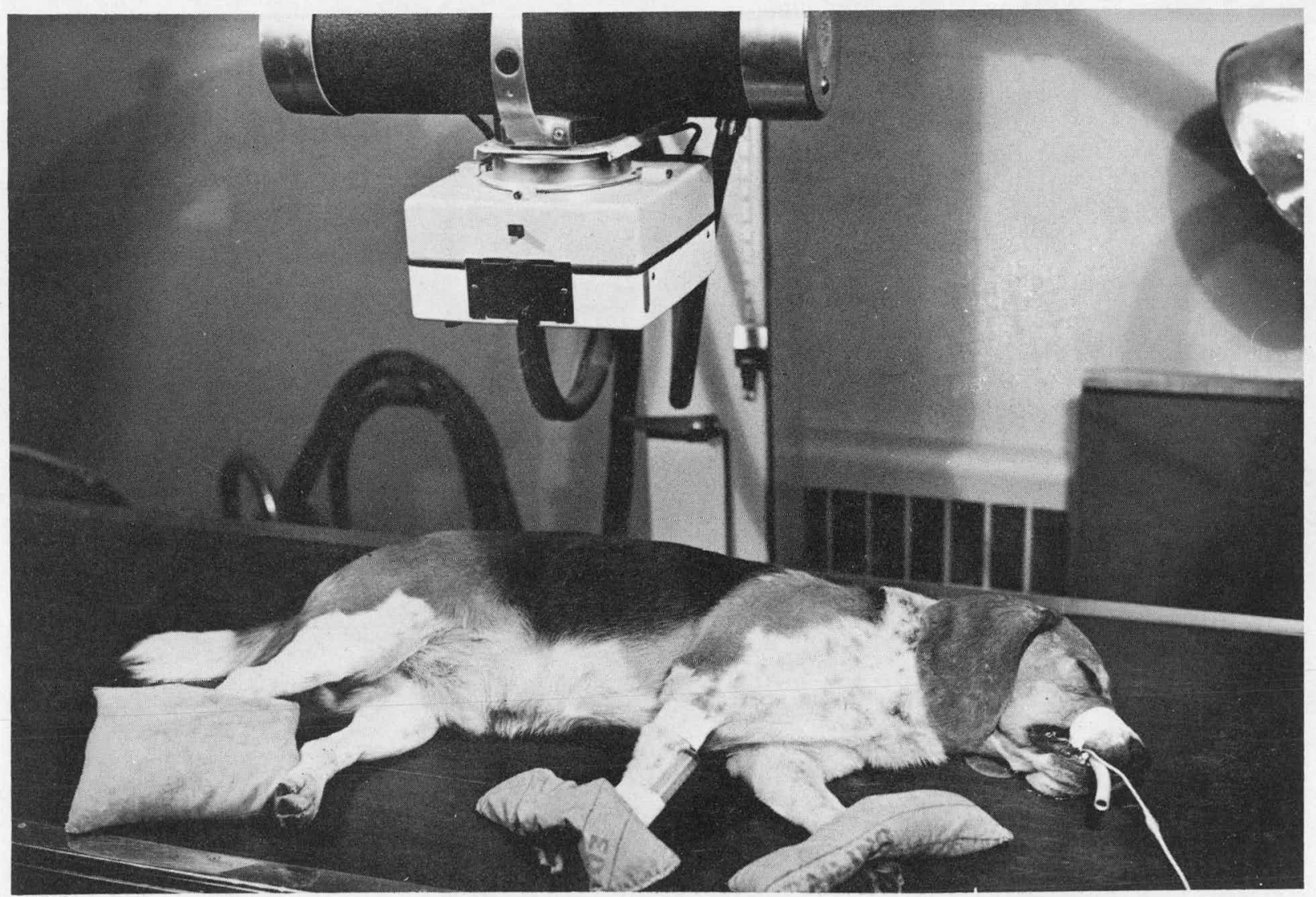

Fig. 1. Anesthetized beagle positioned for radiography. The placement of the syringe with needle in the cephalic vein and the endotracheal catheter is shown. The bags used to "fix" the legs are filled with small lead shot. 


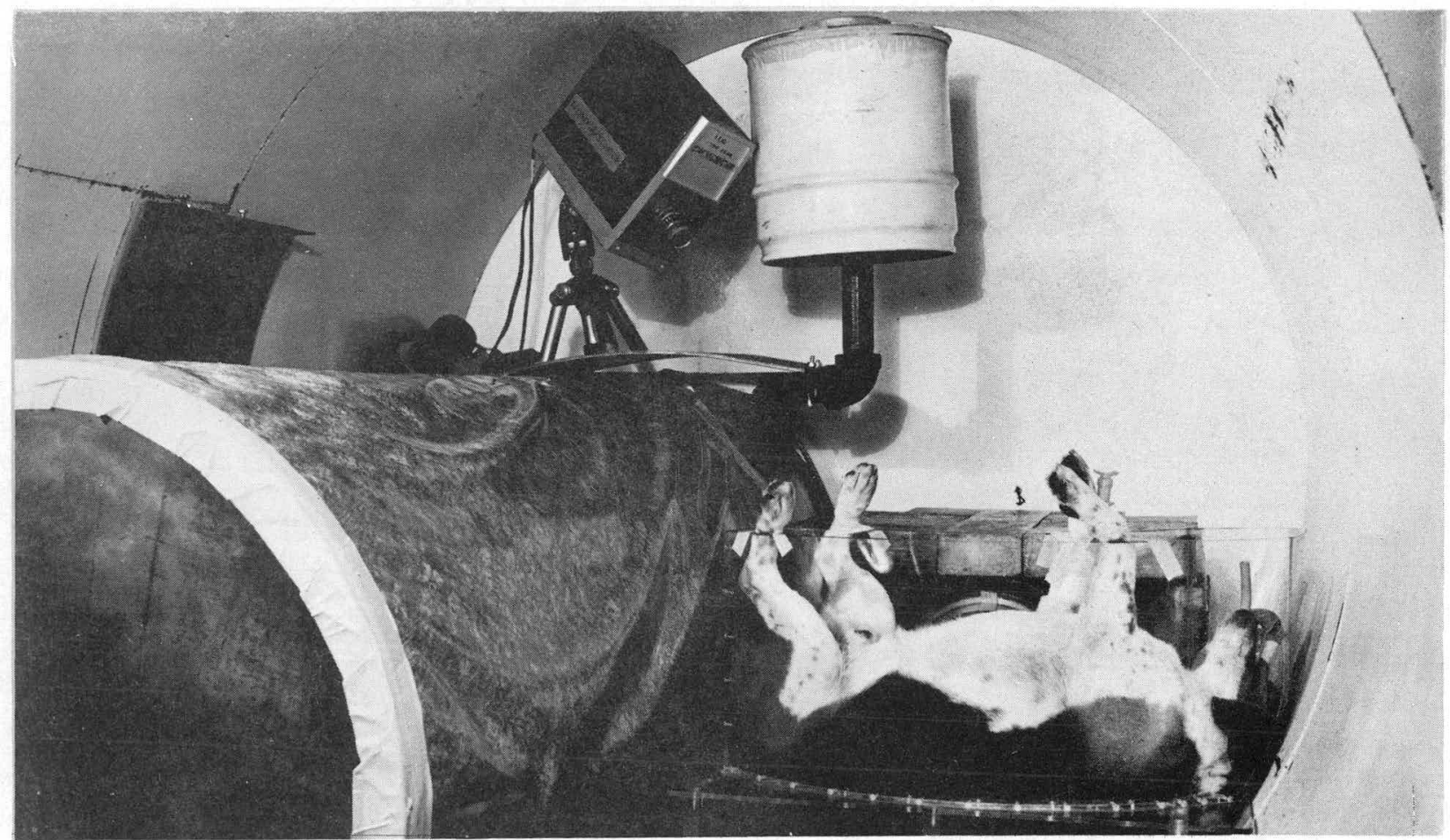

Fig. 2. Anesthetized beagle positioned in plastic holder for whole-body gamma counting. The impracticality of this procedure in an unanesthetized animal is obvious. Note television camera used + monitor dog turing counting interval. (Photo courtesy of Dr. C. W. Mays).

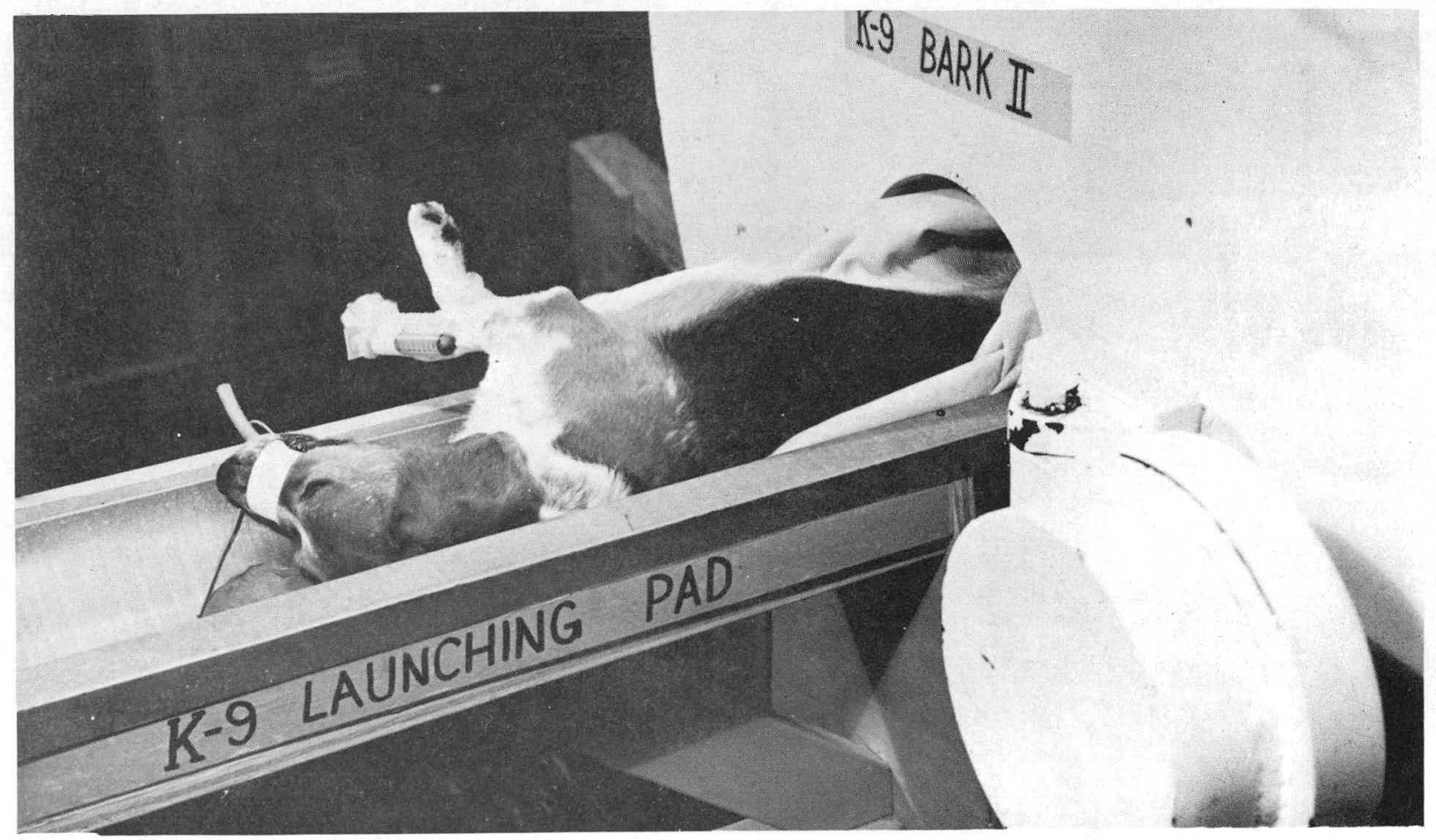

Fig. 3. Anesthetized beagle being inserted into liquid scintillator counter. Again, this procedure would not be practical in the unanesthetized animal. (Photo courtesy of Dr. C. W. Mays) . 


\section{REFERENCES}

1. L. Meyer Jones, Veterinary Pharmacology and Therapeutics, Iowa State College Press, Ames, Iowa, pp. 850 (1954), (See pages 127-130).

2. Louis Goodman and Alfred Gilman, The Pharmacological Bas is of Therapeutics, Macmillan Co., New York, pp. 1387 (1941), (See pages 35-45).

3. J. Dougherty, personal communication (1964) .

4. D. Beriliner, personal communication (1964) .

5. T. T. Currie and W. H. Cole, Methoxyfluorane: A Report of 300 Cases of Clinical Anesthesia, Med. J. Aust. 49: 1009-1011 (1962).

6. V. Keating, Anesthetic Accidents, Year Book Publishers, Inc., Chicago, pp. 288 (1961).

7. J. D. Taylor, R. K. 'Richards and D. L. Tabern, Metabolism of S-Thiopental (pentathal), J. Pharmacol. and Exper. Therap., 104:93 (1952).

8. C. H. Walton, J. Saladamando and W. M. Egner, The Effects of Intravenous pentothal sodium with or without inhalation of oxygen on liver function, Anesthetiology, 12: 67-72 (1951).

9. R. M. Waters and N. A. Gillespie, Deaths in the Operating Room, Anesthesiology, 5: 113-128 (1944). 


\title{
LOW NATURAL INCIDENCE OF OSTEOSARCOMAS IN BEAGLES
}

Charles W. Mays and Glenn N. Taylor

\begin{abstract}
The occurrence of osteosarcomas in non-irradiated beagles is extremely low, as it also is in man. Based on a literature search and inquires to veterinary colleges, the fraction of deaths with natural osteosarcomas for beagles in an environment favoring long life is estimated at 1 to 100 osteosarcomas per 100,000 deaths.
\end{abstract}

To date in our basic program, 192 radioactive beagles have died. Osteosarcomas were diagnosed in 135 (or 70\%). Is the natural incidence of this bone cancer so low in the beagle that every one of these osteosarçomas can be ascribed. to internal irradiation? The following study gives an answer of "yes".

Osteosarcomas are an infrequent form of cancer in the dog. Mulligan ${ }^{(1)}$ lists only 5 osteosarcomas out of 1000 consecutive canine neoplasms, of which about 450 were malignant. Cotchin ${ }^{(2)}$ found 26 osteosarcomas in examining 820 canine neoplasms.

Canine osteosarcomas are especially uncomnon among the smaller breeds ${ }^{(2-4)}$. The following osteosarcoma frequencies were computed (Table 1), using the tumor and admission data of Brodey et al. (3) and the weight clässifications of Davis ${ }^{(5)}$, assuming that the weight distributions for mixed and unspecified breeds were similar to those for the listed breeds. 
TABLE I

OSTEOSARCOMA FREQUENCY BY DOG. WEIGHT

(Computed from tumor and admission data of Brodey and weight classifications of Davis.)

\begin{tabular}{rrrr}
$\begin{array}{c}\text { Weight } \\
\text { (lbs.) }\end{array}$ & $\begin{array}{c}\text { Approx. Admissions at } \\
\text { U. Penn. Vet. School }\end{array}$ & $\begin{array}{c}\text { Number } \\
\text { Osteosarcomas }\end{array}$ & $\left.\begin{array}{c}\text { Admissions } \\
\text { Osteosarcoma }\end{array}\right)$ \\
$5-15$ & 3269 & 0 & $\infty$ \\
$15-30$ & 24072 & 6 & 4012 \\
$30-80$ & 22548 & 92 & 245 \\
Over 100 & 861 & 32 & 27 \\
Total & 50750 & 130 & 390 \\
\hline
\end{tabular}

Having found no natural beagle osteosarcomas in the veterinary .. Iiterature $(1-4)$, we sent inquiries to 26 veterinary colleges requesting (a) their approximate number of beagle admissions, (b) the number of beagles with osteosarcomas, and (c) the name of anyone believed to know of a beagle osteosarcoma. The 20 replies as of 30 October 1964 revealed only 2 beagle osteosarcomas: one at Texas A. \& M. reported by Dr. J. A. Bowen, and the other at Michigan State University reported by Drs. W. O. Brinker, W. A. Priester, and R. A. Tjalma.

The respondents estimated their summed yearly rate of beagle admissions at over 4,000 per year. Yearly registrations of the American Kennel Club ${ }^{(6)}$ indicate that the average size of the registered beagle population during the past 15 years is close to its present.value*. .

*In 1963 there were 49,769 beagle registrations; from 1959 thru 1963 an average of 53,583 beagle registrations per year; and from 1949 thru 1963 an average of 50,593 beagle registrations per year(6). 
Assuming these veterinary colleges have kept complete records during the last 5-15 years, the corresponding total number of their beagle admissions is then about 20,000 for a 5 year period, or 60,000 for a 15 year period. The resulting ratio of 10,000-30,000 beagle admissions per beagle osteosarcoma indicates that the beagle is much less susceptible to osteosarcomas than the average dog (Table I).

Using these data, upper and lower limits can be computed for the incidence of osteosarcomas in beagles allowed to live out their natural lifetimes.

\section{FOR AN UPPER LIMIT ASSUME:}

(a) $100 \%$ will die with neoplasms,

(b) the general dog population has 26 osteosarcomas/820 neoplasms ${ }^{(2)}$,

(c) the beagle is $390 / 10,000$ (or $1 / 26$ ) as susceptible to osteosarcomas as is the average dog (Table I and our Veterinary College surveỳ) .

Upper limit on incidence of beagle osteosarcomas

$=\left[\frac{100 \text { neoplasms }}{100 \text { deaths }}\right]\left[\frac{26 \text { dog osteosar. }}{820 \text { dog neopla. }}\right]\left[\frac{390 \text { dog admission/dog osteosar. }}{10,000 \text { beagle adm/béagle osteosar. }}\right]$ $=\left[\frac{1 \text { beagle osteosarcomas }}{1,000 \text { beagle deaths }}\right]$ or $10^{-3}$

\section{FOR A LOWER LIMIT ASSUME:}

(a) $25 \%$ will die with neoplasms*

(b) the general dog population has 5 osteosarcomas/1000 neoplasms ${ }^{(1)}$,

* Including those groups of beagles showing severe radiation effects (such as osteosarcomas), our colony has had 48 deaths of which 12 (or 25\%) have been with non-skeletal cancers. Cancers of the soft tissues are expected to become a more prominent form of death as our animals grow older. 
(c) the beagle is $390 / 30,000$ (or 1/77) as susceptible to osteosarcomas as is the average dog.

Lower limit on incidence of beagle osteosarcomas $=\left[\frac{25 \text { neoplasms }}{100 \text { deaths }}\right]\left[\frac{5 \text { dog osteosar. }}{1000 \text { dog neopla. }}\right]\left[\frac{390 \text { dog admission/dog osteosar. }}{30,000 \text { beagle adm./beagle osteosar. }}\right]$ $=\left[\frac{1 \text { beagle osteosarcoma }}{100,000 \text { beagle deaths }}\right]$ or $10^{-5}$

\section{DISCUSSION}

We have computed upper and lower limits to emphasize that the data available do not permit exact calculation of the true incidence. Limitations exist in any questionaire survey, and there are uncertainties in how beagles compare with the general canine population in age distribution; age at osteosarcoma diagnosis, and reasons for admission to veterinary colleges. Taken at face value, these limits indicate that roughly $1,000-100,000$ beagles of long lifespan would be required to produce a single spontaneous osteosarcoma 。

At the Long Term Effects session of the 1964 Radiation Research Society meeting we asked investigators studyịng beagles (at Davis, California; the Argonne National Laboratory; Fort Collins, Colorado; Rochester, New York; Richland, Washington; and Albuquerque, New Mexico) if they were aware of even 1 osteosarcoma in a non-irradiated beagle inside or outside of their colonies. No cases were indicated, nor have we observed any osteosarcomas among our control beagles. This adds weight to the conclusion that naturally-occurring osteosarcomas are extremely rare in beagles.

This low incidence of natural osteosarcomas makes the beagle an ideal research animal for studying the effects of skeletal irradiation. 
We will eventually have about 200 low-level radioactive dogs on our project whose skeletal dose-rates are probably too small to shorten group lifespans appreciably. The low probability of $[200$ dogs $]$ $\left[\left(10^{-5}\right.\right.$ to $\left.\left.10^{-3}\right) / \mathrm{dog}\right]=(0.002$ to 0.2$)$ that a single osteosarcoma would arise spontaneously in a group this size gives confidence that each osteosarcoma which developes can be ascribed to irradiation. In the same number of heavily irradiated dogs, the chance occurrence of a spontaneous osteosarcoma is even lower due to the shortened lifespan available for such effects to appear.

It is interesting that the osteosarcoma/death ratio in man* of about $2 \times 10^{-4}$ falls within the range of $10^{-5}$ to $10^{-3}$ calculated for the beagle. Relative biological effectiveness values among radionuclides in producing osteosarcomas in the beagle can be extrapolated to man with added reliance.

\section{ACKNOWLEDGEMENTS}

Our sincere appreciation is extended to the following individuals and institutions whose help made this study possible: R. H. Carlberg, American Kennel Club; Jean Flipo, University of Montreal; W. 0. Brinker and R. A. Tjalma, Michigan State University; W. A. Priester, National Institute of Health; B. F. Hoerlein, Alabama Polytechnic Inst.; W. J. Roenigh, Ohio State University; G. W. Mather, University of Minnesota; R. S. Brodey, University of Pennsylvania; R. G. Buckner,

*In 1962 there were 1750 reported "bone" cancers in a total of 1,757,000 deaths in the U. S. A. (7) However, not all "bone" cancers reported on death certificates are osteosarcomas. A study by Auerbach $(8)$ revealed only 35 osteosarcomas in 166 "death certificate reported" bone cancers.

$\left[\frac{1750 \text { rept. bone ca. }}{1,757,000 \text { deaths }}\right]\left[\frac{35 \text { osteosarcomas }}{166 \text { rept. bone ca. }}\right]=\frac{2 \text { human osteosarcomas }}{10,000 \text { human deaths }}$ 
Oklahoma State University; C. Faus, University of Cambridge; E. Cotchin, Royal Vet. College, London; K. H. Niemeyer, University of Missouri; D. L. Baker, Iowa State University; J. A. Bowen, Texas A. \& M.; A. G. Shiller, University of Illinois; R. W. Kirk, Cornell University; K. Smith, Colorado A.\& M.; G. D. Pettit, Washington State University; J. E. Mosier, Kansas State University; J. K. Kealy, University of Ireland; J: M. Keef, University of Sidney, Australia; A. M. Brues, Georgia DeRoche and H. Auerbach, Argonne National Laboratory; E. M. Jones, Midwest City, Oklahoma; A. J. Cawley, University of Toronto.

\section{REFERENCES}

1. R. M. Mulligan, Neoplasms of the Dog, Williams and Wilkins Co:, Bàltimore, 135 pages (1949) see page 4.

2. E. Cotchin, Spontaneous sarcomas of bone in dogs: 30 cases, Brit. Vet. Jour. 109, 248-257 (1953) see page 248 and 252 .

3. R. S. Brodey, R. M. Sauer, and W. Medway, Canine bone neoplasms, J. Am. Vet. Med. Assoc. 143:5, $471-495$ (1 September 1963).

4. N. L. Owen and D.E. Stevenson, Observations on canine osteosarcomata, Res. Vet. Sci. 2, 117-129 (1961). 5. H. P. Davis, The Dog Encyclopedia, Stackpoie Co., Harrisburg
(1953), see pages 514-516.

6. Roy H. Carlberg, letter of 18 September 1964 to Glenn N. Taylor.

7. Facts on thecmajor killing and crippling diseases in the United States today, compiled by the National Health Education Committee, Inc., 135 East 42nd Street, New York City, (1964) see pages 4 and 31 .

8. H. Auerbach, Illinois bone tumor death certificate study, Argonne National Laboratory Medical and Research Division Report ANL-6264, 7-8 (July-December 1959); and personal communications from A. M. Brues and Georgia De Roche for H. Auerbach. 


\title{
THE EFFECTS OF IONIZING RADIATIONS ON ENDOCRINE CELLS
}

IV. INCREASED .PRODUCTION OF $17 \alpha, 20 \alpha$-DIHYDROXYPROGESTERONE BY

RAT TESTES AFTER IRRADIATTON

\author{
David L. Berliner ${ }^{l}$ and LeGrande C. Ellis ${ }^{2}$
}

Abstract: Rat testicular mince was incubated with progesterone-4-CI4. From the incubation mixture, $17 \alpha, 20 \alpha$-dihydroxyprogesterone was isolated and characterized as one of the main steroids of the high-polar steroid fraction. The biosynthesis of this steroid has been shown to be altered after various modes of irradiation. When $17 \alpha, 20 \alpha$-dihydroxyprogesterone was incubated with mouse testicular tissue, only small amounts of this steroid were biotransformed into $17 \alpha-$ hydroxyprogesterone, androstenedione and testosterone. These data indicate that an accumulation of a non-androgenic steroid occurs after direct irradiation to the testes.

Previous investigations on the biotransformation of 5 -pregnenolone ${ }^{3}$ and progesterone into androgens by mouse $(1)$ and rat $(2,3)$ testes have shown that there are considerable amounts of these two substrates that are biotransformed into steroids with a polarity greater than testosterone. Furthermore, the amount of 5-pregnenolone and progesterone being converted into these so-designated "high-polar" steroids was altered following either localized irradiation to the testes (2) or irradiation to the heads (3) of rats. This paper describes the biosynthesis, isolation, and characterization of one of these high-polar steroid compounds, and the subsequent biotransformation of this compound into testosterone and its precursors by murine testicular tissue. The relationship between the biosynthesis of this high-polar steroid with irradiation and aging is also discussed in this paper.

MATERIALS AND METHODS

A large quantity of $17 \alpha, 20 \alpha$-dihydroxyprogesterone was biosynthesized according to the following procedure. Twenty-six male rats (Sprague- 
Dawley strain) were euthenized when 14 weeks of age by light ether anesthesia and cervical dislocation. The testes from these animals were quickly removed and chilled in ice-cold phosphate buffer, pH 7.4. After being trimmed and weighed, the testes were finely minced with razor blades. Progesterone-4-c $\mathrm{C}^{14}$ (New England Nuclear. Co.) was purified by paper chromatography (4) and adjusted to a specific activity of $10 \mathrm{mc} /$ $10 \mathrm{mg}$ with unlabeled progesterone. The $\mu \mathrm{c}(10 \mathrm{mg})$ of this mixture in $99 \mathrm{ml}$ of methanol and $1 \mathrm{ml}$ of propylene glycol were transferred to a four liter Erlenmeyer flask. The methanol was blown off with a stream of nitrogen and 77.5 grams of testicular mince, $800 \mathrm{ml}$ of phosphate buffer ( $\mathrm{pH}$ 7.4), and $100 \mu$ moles each of NADPH and glucose-6-phosphate (Sigma Chemical Co.) were transferred to the flask. The contents of the flask were incubated for three hours under atmospheric air at $37.5^{\circ} \mathrm{C}$ in a constant temperature bath (American Instrument Co.). At this time, the reaction was stopped, and the steroids were extracted and isolated as described elsewhere $(1,5)$. Characterization and identification of $17 \alpha$, 20 $\alpha$-dihydroxyprogesterone were accomplished throügh paper chromatography and derivative formation (acetylation with acetic anhydride and oxidation with chromic acid) (5) . Steroids on the paper chromatograms were visualized with an ultraviolet scanner and an electronic (Geiger-Mueller) scanner (6)

In order to ascertain if $17 \alpha, 20 \alpha$-dihydroxyprogesterone could be biotransformed into androgens, 92,952 CPM of this steroid were incubated with 0.533 grams of testicular mince from $C B A$ mice (14 weeks of age) in $10 \mathrm{ml}$ of phosphate buffer, $\mathrm{pH} 7.4$. This mixture was incubated $21 / 2$ hours at $37.5^{\circ} \mathrm{C}$ under atomospheric air. No NADPH-generating system was 
used with this incubation. Testosterone and its immediate precursors were isolated and characterized as previously described in the literature (1) .

\section{RESULTS}

A large quantity of progesterone was separated from the steroid mixture after chromatographing the steroid extract in the hexane: formamide system. Similarly, androstenedione, testosterone, and 17 $\alpha$-hydroxyprogesterone were separated from the steroid exEract after chromatographing it in the hexane-benzene: formamide system. Steroids from this second chromatogram with an $\mathrm{Rf}$ less than 0.30 were eluted from the chromatogram and rechromatographed in the benzene: formamide system. A large quantity of ultraviolet positive, radioactive labeled steroid with an $\mathrm{Rf}$ of 0.29 was observed. This compound was identified as $17 \alpha, 20 \alpha$-dihydroxyprogesterone on the basis of the following characteristics: a) the presence of $\Delta^{4}-3$-keto group as demonstrated by an ultraviolet spot on the chromatogram and confirmed by an absorption maximum of $240 \mathrm{~m} \mu$ as observed with a Ratio Recording Spectometer (Beckman Inst. Co.), b) the presence of only one acetylatable group, which disappeared when an aliquot of the steroid was oxidized prior to acetylation, c) no change in mobility when the steroid acetate was subjected to mild oxidation with chromic acid, d) the products formed after mild oxidation of the free steroid with chromic acid were $17 \alpha$-hydroxyprogesterone and androstenedione, and e) the inversion of polarity of this compound with respect to its isomer $17 \alpha, 20 \beta$-dihydroxyprogesterone upon acetylation which is typical for $20 \alpha-$ and 20ß-hydroxylated steroids (5) .

When the original material having an $\mathrm{Rf} 0.29$ in the benzene: formide 
system was incubated with mouse testicular tissue, the following steroids were isolated (Table I) : 17 -hydroxyprogesterone, androstenedione, and testosterone.

The amounts of high-polar compounds formed after direct irradiation of the testes and heads of rats are recorded in Table II along with their respective probability statements.

\section{DISCUSSION}

The isolation and characterization of $17 \alpha, 20 \alpha$-dihydroxyprogesterone from incubations of rat testicular tissue show that this tissue is capa-

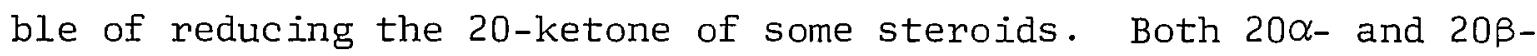
reductases have been previously observed in human, fetal testicular tissue (7), and avian testicular tissue (8). Dominquez et al. (9) reported the isolation of the $20 \beta$-isomers of progesterone in normal rat testes. Only a small amount of 17 $\alpha, 20 \beta$-dihydroxyprogesterone was observed in this investigation, but $12.6 \%$ of the progesterone in the original incubation mixture was converted into $17 \alpha, 20 \alpha$-dihydroxyprogesterone.

Although the physiologic role of $20 \alpha$-reductase is not known for the testes, the presence of this enzyme has been reported in malignant tumor tissue (9). Moreover; in avian testes (10) there appear to be four.20reductase enzymes (8), which appear to be located in the germinal elements of the testis. These workers (10) observed a small increase in the activity of both $20 \alpha$ - and $20 \beta$-reductase enzymes, which was associated with gonadotrophin injections. However, there was a more marked increase in the activity of these two enzymes concomitant with an increase in the germinal elements during the normal cycling of the English Sparrow. Other data indicate that there is an increase in 20-reductase activity 
associated with aging (L. R. Axelrod - personal commuication) .

The data from this investigation show that $17 \alpha, 20 \alpha$-dihydroxyprogesterone is one of the main steroids of the high-polar steroid fraction; and, therefore, fluctuates after irradiation $(2,3)$. When rat testes were directly irradiated with $1500 \mathrm{r}$ of $\mathrm{x}$-rays, there was a decrease in the ability of this tissue to biotransform steroids into androgens 20 days after treatment (2). There was, however, an increase in the amount of $17 \alpha, 20 \alpha$-dihydroxyprogesterone produced per $\mathrm{mg}$ of tissue used in the incubations (Table II). On the other hand, the amount of this compound produced by testicular tissue from head-irradiated rats was decreased (Table II) by this treatment. The difference in response noted in these two investigations is probably due to the difference in the mode of administration of the irradiation. It would appear from these data that the in vitro biosynthesis of $17 \alpha, 20 \alpha$-dihydroxyprogesterone is related to the physiologic state of the testis.

The data from this investigation (Table I) show that $17 \alpha, 20 \alpha$-dihy-: droxyprogesterone can be reversibly biotransformed into $17 \alpha$-hydroxyprogesterone, androstenedione and testosterone. The probable sequence for these biotransformations appears in Fig. 1 . It would appear from these data (Table I) that side-chain cleavage of $17 \alpha, 20 \alpha$-dihydroxyprogesterone into androstenedione represents the minor of two possible routes for the biotransformation of this high-polar compound into androgens. The slow rate of conversion of $17 \alpha-20 \alpha$-dihydroxyprogesterone into androgens undoubtedly reflects the low biological activity of 20-hydroxylated derivatives of 17 $\alpha$-hydroxyprogesterone (11) and progesterone (12) . 
TABLE I

The Conversion of $17 \alpha, 20 \alpha$-Dihydroxyprogesterone into Testosterone by Mouse Testes

\begin{tabular}{lcc}
\hline $\begin{array}{l}\text { Steroid } \\
\text { Isolated }\end{array}$ & $\begin{array}{l}\text { Counts } \\
\text { Per Minute }\end{array}$ & $\begin{array}{l}\text { Percent } \\
\text { Conversion }\end{array}$ \\
$17 \alpha, 20 \alpha$-dihydroxyprogesterone & 92,952 & \\
$17 \alpha$-hydroxyprogesterone & 2,150 & $2.30 \%$ \\
Androstenedione & 250 & $0.26 \%$ \\
Testosterone & 34 & $0.03 \%$ \\
\hline
\end{tabular}

TABLE II

Amounts of $17 \alpha, 20 \alpha-D$ ihydroxyprogesterone Formed

by Rat Testicular Tissue Following Various Modes

of: Irradiation

Experimental

Group

Localized irradiation of testes

$\begin{array}{lll}\text { Control } & 13.45 \pm 1.5^{*} & \text { P } 0.05^{* *} \\ \text { Irradiated },(1500 \mathrm{r}) & 28.25 \pm 4.70\end{array}$

Head irradiated rats

Control

Irradiated

* Standard error of mean.

** Statistical comparisons were made by using a " $t$ " test. 

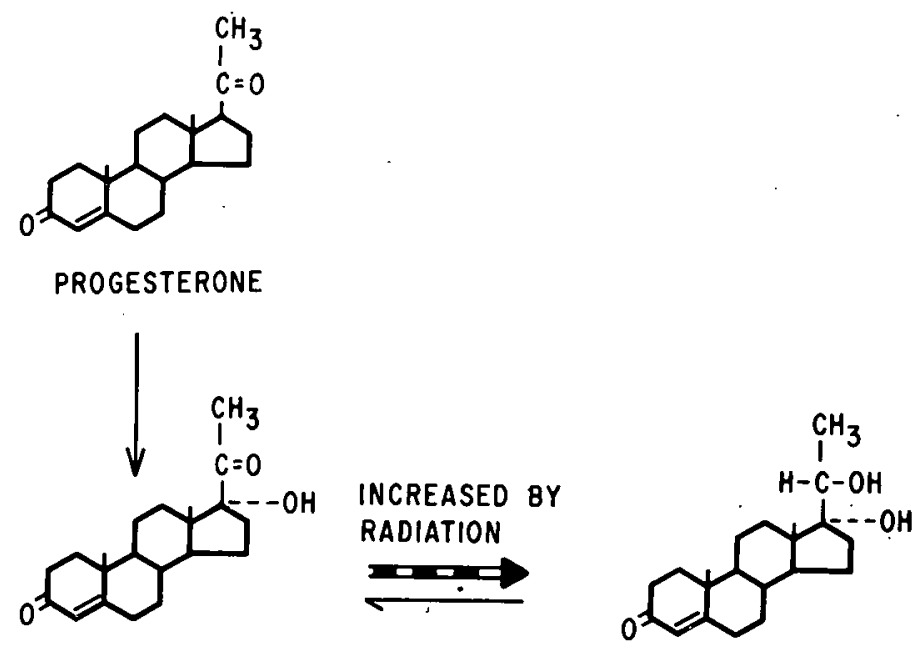

$17 \propto$ HYOROXYPROGESTERONE

$17 \alpha, 20 \alpha$-DIHYDROXYPROGESTERONE

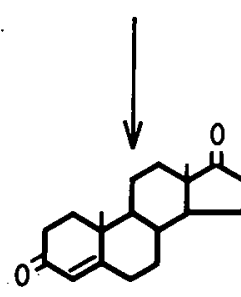

ANDROSTENEDIONE
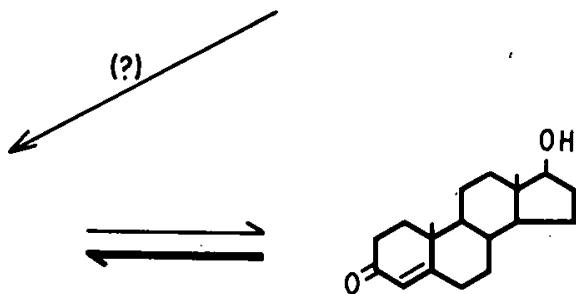

TESTOSTERONE

Fig. 1. Proposed pathway for, the synthes is of $17 \alpha, 20 \alpha$-dihydroxyprogesterone and the subsequerit conversion of this compound to androgens. 


\section{FOOTNOTES}

${ }^{1}$ U.S.P.H.S. Research Career Development Award (1-K6-CA-22, 571-01) .

${ }^{2}$ U.S.P.H.S. Fellow (Training Grant \#5Tl GM-958-03) • Present address: Department of Zoology, Utah State University, Logan, Utah.

${ }^{3}$ In this paper, the following abbreviations are used: 5-pregnenolone,

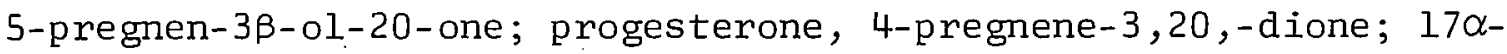
hydroxyprogesterone, 4-pregnen-17 $\alpha$-ol-3,20-dione; androstenedione, 4-androstene-3,17-dione; testosterone, 4-androsten-17B-ol-3-one; $17 \alpha, 20 \alpha$-d ihydroxyprogesterone, 4-pregnene-17 $\alpha, 20 \alpha$-diol-3-one; $17 \alpha$,

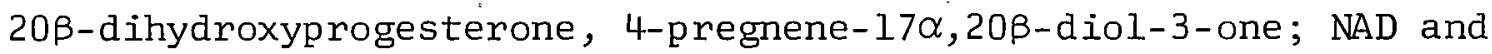
$\mathrm{NADH}$, oxidized and reduced forms of nicotinamide adenine dinucleotide; and NADP and NADPH, oxidized. and reduced forms of nicotinamide adenine dinucleotide phosphate.

\section{REFERENCES}

1. L. C. Ellis and D. L. Berliner, The effects of ionizing radiations on endocrine cells. I. Steroid biotransformations and androgen production by testes from irradiated mice. Radiation Res. 20:549-563 (1963)

2. D. L. Berliner, L. C. Ellis, and G. N. Taylor, The effects of ionizing radiations on endocrine cells. II. Restoration of androgen production with a reduced nicotinamide adenine dinucleotide phosphategenerating system following irradiation of rat testes. Radiation Res. $22: 345-356$ (1964).

3. L. C. Ellis and D. L. Berliner, The effects of ionizing radiations on endocrine cells. III. Restoration of testosterone production with a reduced nicotinamide adenine dinucleotide phosphate-generating system after localized irradiation to heads of rats. Radiation Res. (In press - 1964).

4. A. Zaffaroni, Micromethods for the analysis of adrenocortical steroids. In Recent Progress Hormone Research, Vol. 8, pp. 51-86, Academic Press, Inc., New York, 1953 . 
5. D. L. Berliner, The microchemical identification of steroids from biological media. In Advances In Tracer Methodology, (Ed. S. Rothchild) Vol. II, Plenum Press, New York (In Press - 1964).

6. D. L. Berliner, O. V. Dominquez, and G. Westenskow, Determination of carbon-14 steroids on paper chromatograms. Anal. Chem. 29:1797-1800 (1957) .

7. H. F. Acevedo, L. R. Axelrod, E. Ishikama, and F. Takaki, Studies in fetal metabolism. II. Metabolism of progesterone-4-C 14 and pregnenolone-7 $\alpha-\mathrm{H}^{3}$ in human fetal testes. J. Clin. Endocrinol Metab. 23: 885-890 (1963).

8. H. F. Fevold and K. B. Eik-Nes, Progesterone metabolism by testicular tissue of the English Sparrow (Passer domesticus). Gen. Comp. Endocrinol. 3:335-345 (1963).

9. O. V. Dominquez, H. F. Acevedo, R. A. Huseby and L. T. Samuels, Steroid 2l-hydroxylose in normal testes and malignant interstitial cell tumors, Jour. Biol. Chem. 235:2608-2612 (1960).

10. H. F. Fevold and K. B. Eik-Nes, Progesterone metabolism by testicular tissue of the English Sparrow (Passer domesticus) during the annuál. reproductive cycle. Gen. Comp. Endocrinol. 2:506-515 (1962). .:

11. W. G. Wiest, and T. R. Forbes, Failure of $20 \alpha$-hydroxy- $\Delta^{4}$-pregnen-3one and $20 \beta$-hydroxy- $\Delta^{4}$-pregnen-3-one to maintain pregnancy in ovariectomized mice. Endocrinol. 74:149-150 (1964).

12. C. Lauritzen, Biologische wirkungen des 20ß-hydroxy-pregn-4-en-3-on. Acta Endocrinol. 44:225-236 (1963). 


\title{
SERUM LACTIC DEHYDROGENASE LEVELS \\ IN ADULT BEAGLE DOGS
}

Ann G. Ruhmann and David L. Berliner

\begin{abstract}
Serum lactic dehydrogenase (LDH) determinations were performed on 101 beagles fromi:a colony used in the study of long range effects of internally deposited radionuclides. Statistical analys is of results revealed no significant differences among groups separated by radioisotope injected, number of days elapsed since nuclide injected, and disease state. Sera from moderately hemolyzed blood samples were significantly higher in LDH level than sera from nonhemolyzed or slightly hemolyzed blood samples. Dogs who received high doses of radionuclides had blood LDH levels significantly higher than those of control dogs. It is concluded that serum $\mathrm{LDH}$ is not a consistent indicator of pathologic states in the adult beagle dog.
\end{abstract}

Innumerable reports have been made concerning the correlation, or lack of it, between lactic dehydrogenase (LDH) activity in serum, plasma, or tissue homogenates; and presence of neoplastic disease. Interpretation of reports has been complicated by the use of myriad methods of assay, and testing of many different species. Thus, comparisons between LDH catalyzing pyruvate to lactate. $(1,2,3,4)$, and LDH catalyzing the reverse reaction (5), the many modifications of these procedures, and the differences in the various isozymes $(6,7,8)$ of $\mathrm{LDH}$ must all be con:sidered. Moreover, not only are the normal values for these procedures not comparable, they differ according to species tested for both average values and those found during disease states.

In our laboratory, as well as in others, studies on the long term effects of internally deposited radionuclides are being pursued in a large colony of beagle dogs (9). Since a very important long term effect of radiation is the formation of tumors, a method of detecting the early presence of neoplastic tissue is desirable. It was our purpose, in this study to establish a norm for $\mathrm{LDH}$ in dog serum, and to determine whether 
or not LDH level is a good indicator of the presence, growth, and extent of neoplasia.

\section{METHODS}

Serum was obtained from routine blood letting of colony dogs. The blood was slowly withdrawn through a large gauge needle and slowly emptied into test tubes, standing in ice, care being taken to avoid hemolyzing the erythrocytes. The blood was then spun in a refrigerated centrifuge and the serum removed. At no time between drawing and testing, was the serum allowed to rise above $5^{\circ} \mathrm{C}$. LDH level was determined by the method of Snodgrass (5) at $22.6 \pm 0.3^{\circ} \mathrm{C}$. The temperature was tested inside the cuvette before and after the 3 minute spectrophotometer reading interval.

In general the serum was tested within a few hours of the time it was drawn, although in a few cases, as much as 24 hours elapsed before the LDH level was determined. Samples to be kept longer than 3 hours were frozen. Repeat determinations over 24 hour periods on 15 different frozen samples showed a mean $\mathrm{LDH}$ of 30 units, with an average variation of 4 units and a maximum variation of 10 units (one sample only). The . procedure was tested against Versatol $\mathrm{E}^{1}$, and consistently gave results 20\% higher than the values reported in the brochure accompanying the standared.

\section{RESULTS}

A total of 101 different animals were tested for serum lactic dehydrogenase. Many dogs were tested two and sometimes three separate times,

\footnotetext{
${ }^{l}$ General Diagnostics Division of Warner Chilcott.
} 
but in these cases, only the first determination was used in figuring statistical significance of group differences.

The grand mean for all animals tested was 22.1 units LDH activity.

All of the dogs tested were part of a colony used in the study of internally deposited radionuclides. The radioisotopes included $\mathrm{Th}^{228}$, $\mathrm{Ra}^{224}, \mathrm{Ra}^{226}, \mathrm{Ra}^{228}, \mathrm{Sr}^{90}$ and $\mathrm{Pu}^{239}$, all of which had been injected intravenously as a single dose of varying levels of radioactivity (9). Table. $I$ is a summary of the LDH values of the dogs, using isotope as the factor of variation. Although the mean values ranged from $17.5 \pm 4.5$ to $34.0 \pm$ 6.1 , an analysis of variance revealed that there were no significant differences among the groups $(F=1.3)$.

Time elapsed since radioisotope injection was considered as another possible parameter of variation. Since all animals were injected at approximately 500 days of age, this not only correlated with length of time in contact with radionuclide, but also with the dog's actual age. Dogs were separated into four groups, by days post-injection: (a) 0-500, (b) 501-2000, (c) 2001-3000, (d) more than 3000. Table II summarizes this analysis. Pre-injection dogs were not included in this analysis, since group (a) was a good control group of comparable age. Although the older dogs have an average LDH level well below the others, the sample size is, small and the standard deviation large. The difference is not significant $(F=0.66)$.

A comparison between animals which were clinically well and showed no abnormalities by chemical or hematological laboratory procedures and those which had tumors or other abnormalities was undertaken. Reference to Table III shows the animals to be divided into 5 groups: (a) normal, (b) those with a clinical diagnosis of tumor, probably benign, (c) those with 
TABLE I

LDH Values on Radioisotope Burdened Dogs

\begin{tabular}{lcccc}
\hline \hline & \multicolumn{3}{c}{ Mean } \\
LDH Units & S. D. & F \\
\hline Control & 35 & 19.7 & 3.3 & 5.2 \\
$\operatorname{Th}^{228}$ & 14 & 23.9 & 7.4 \\
$\operatorname{Ra}^{224}$ & 7 & 24.9 & 4.5 \\
$\operatorname{Ra}^{226}$ & 19 & 17.5 & 6.2 \\
$\operatorname{Ra}^{228}$ & 10 & 31.6 & 6.1 \\
$\mathrm{Sr}^{90}$ & 10 & 34.0 & 5.0 \\
$\mathrm{Pu}^{239}$ & 15 & 21.6 & \\
$\operatorname{Total}^{23}$ & 110 & 22.8 & 1.3 \\
\hline
\end{tabular}

TABLE II

$\mathrm{LDH}$ Values on Dogs at Various Intervals After Injection

\begin{tabular}{|c|c|c|c|c|}
\hline Days PI & $n$ & $\begin{array}{c}\text { Mean } \\
\text { LDH Units } \\
\text { Per Dọg }\end{array}$ & S. D. & $\mathrm{F}$ \\
\hline $0-500$ & 28 & 24.1 & 4.1 & \\
\hline $501-2000$ & 18 & 26.8 & 5.1 & \\
\hline $2001-3000$ & 16 & 25.0 & 5.4 & \\
\hline Over 3000 & 8 & 14.2 & 7.6 & \\
\hline Total & 70 & 23.9 & & 0.66 \\
\hline
\end{tabular}


histologically diagnosed benign tumors, (d) those with histologically identified malignant tumors, and (e) those with abnormal hematological or blood chemical changes, or clinical disease other than tumor. There is tremendous individual variation between animals in the same group, as reflected in the large standard deviations. Perusal of the data reveals no trend toward higher or lower $L D H$ values in any group, and this is born out by the analysis of variance, which indicates that the sick and well dogs comprise approximately the same population.

Owing to the technical problems involved in bleeding large numbers of dogs daily, plus the extreme fragility of the beagle red blood cell:, the study included a small percentage of hemolyzed sera. All samples, therefore, were visually compared to a color chart and classified as 0 through $5+$ hemolysis. Five sets of samples were analyzed for oxyhemoglobin in a Beckman DU spectrophotometer at $540 \mathrm{~m} \mu$ to establish the approximate range and relative accuracy of the visual method. Sera classified between 0 and $2+$ by the visual method varied from 0.010 gm \% $\mathrm{Hb}$ to $0.018 \mathrm{gm} \%$, with the great majority falling between 0.016 and $0.018 \mathrm{gm} \%$. Sera classified as $3+$ measured at 0.038 to $0.042 \mathrm{gm} \%$ hemoglobin. Bloods hemolyzed to a greater extent than $3+$ were discarded without being assayed for LDH. Table IV presents the statistical analysis of the LDH determinations using degree of hemolysis as the factor of variation. The F ratio of 4.3 is significant, indicating that the means are from different populations. Individual t-tests, using the 0 hemolysis group as the control, indicate that the $3+$ group is significantly higher in serum LDH at the $0.02-0.05$ level $(t=2.35)$.

Because of the high mortality among dogs receiving large doses of radioactivity, only $0,1,2$, and 3 level (9) dogs were available for $\mathrm{LDH}$ 
TABLE III

LDH Values on Healthy and Sick Dogs

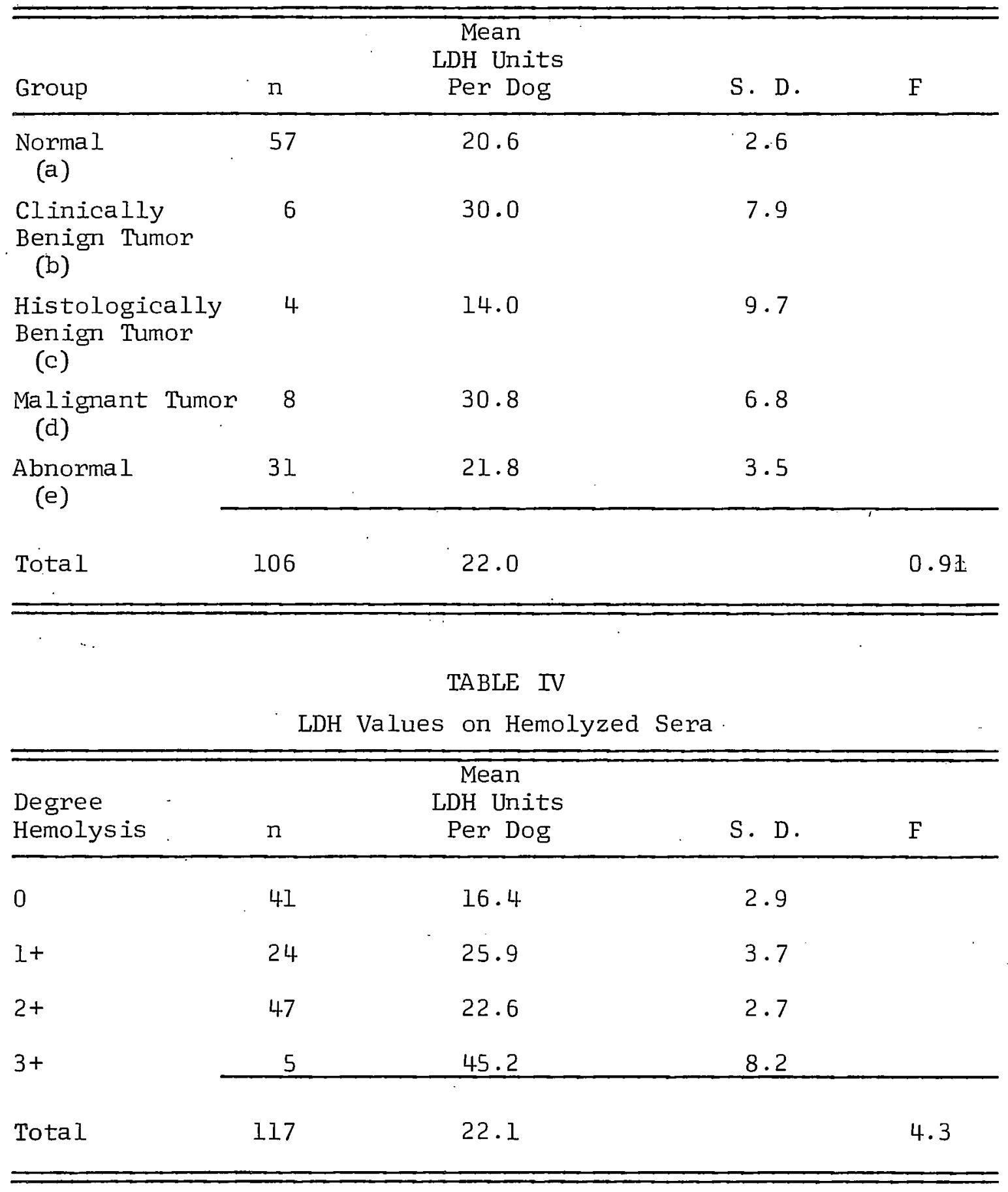


assay. Small sample sizes made analysis by isotope impossible, but $t-$ tests were performed comparing 0-level (placebo injection) to each of the 1, 2, and 3 level groups. Level 3 animals received approximately 3 times the dose $(\mu \mathrm{c} / \mathrm{kg})$ given 2-level animals, and 20 times that given l-level animals. The numbers of values from each isotope group were roughly the same for each dose level. Reference to Table $V$ reveals that the 3-level beagles had significantly higher serum LDH values: than the control group. Table VI is a compilation of all the individual LDH determinations.

\section{DISCUSSION}

The mean $\mathrm{LDH}$ value of 22.1 units for 117 dogs tested, is considerably lower than the average value of 59 units obtained by Snodgrass et al. (5), using human serum. 'Interspecies differences are well known in all enzyme systems, and this result merely reinforces the need to establish norms for the species being considered, as well as for the laboratory in which the work is being. done.

The results of the testing of animals receiving different radionuclides were not remarkable. These animals had. been-initially chosen for injection because of comparability of age, lineage, and health. Most of the high level animals in all groups succumb to osteosarcoma, and although the number of osteosarcomas, as well as cases of hepatic necrosis, is largest in the $\mathrm{Pu}^{239}$ dogs $(9,10)$, this was not reflected in any alteration of serum LDH. However, it must be remembered that our samples were definitely weighted to the low dose side and that many of. the high level animals died before this study was initiated.

Because animal age is found to effect some enzyme levels, and most radioisotope effects manifest themselves mainly after long term exposure, 
TABLE $\mathrm{V}$

t-test Analys is of Serum LDH

in Dogs Which Received Different Doses of Radionuclides

\begin{tabular}{|c|c|c|c|c|c|}
\hline Group & $\mathrm{n}$ & $\begin{array}{l}\text { Mean } \\
\text { LDH Units } \\
\text { Per Dog }\end{array}$ & $\mathrm{t}: \because$ & & $\mathrm{P}$ \\
\hline 0 -level & 14 & 15.7 & & & \\
\hline 1-level & 12 & 20.8 & 0.68 & $\therefore$ & 0.5 \\
\hline 2-level & 8 & 16.5 & 0.15 & $\therefore$ & $0.8-0.9$ \\
\hline 3 -level & 8 & 56.0 & 2.90 & & $<0.01$ \\
\hline
\end{tabular}

TABLE VI

Individual LDH Values on All Beagles Tested

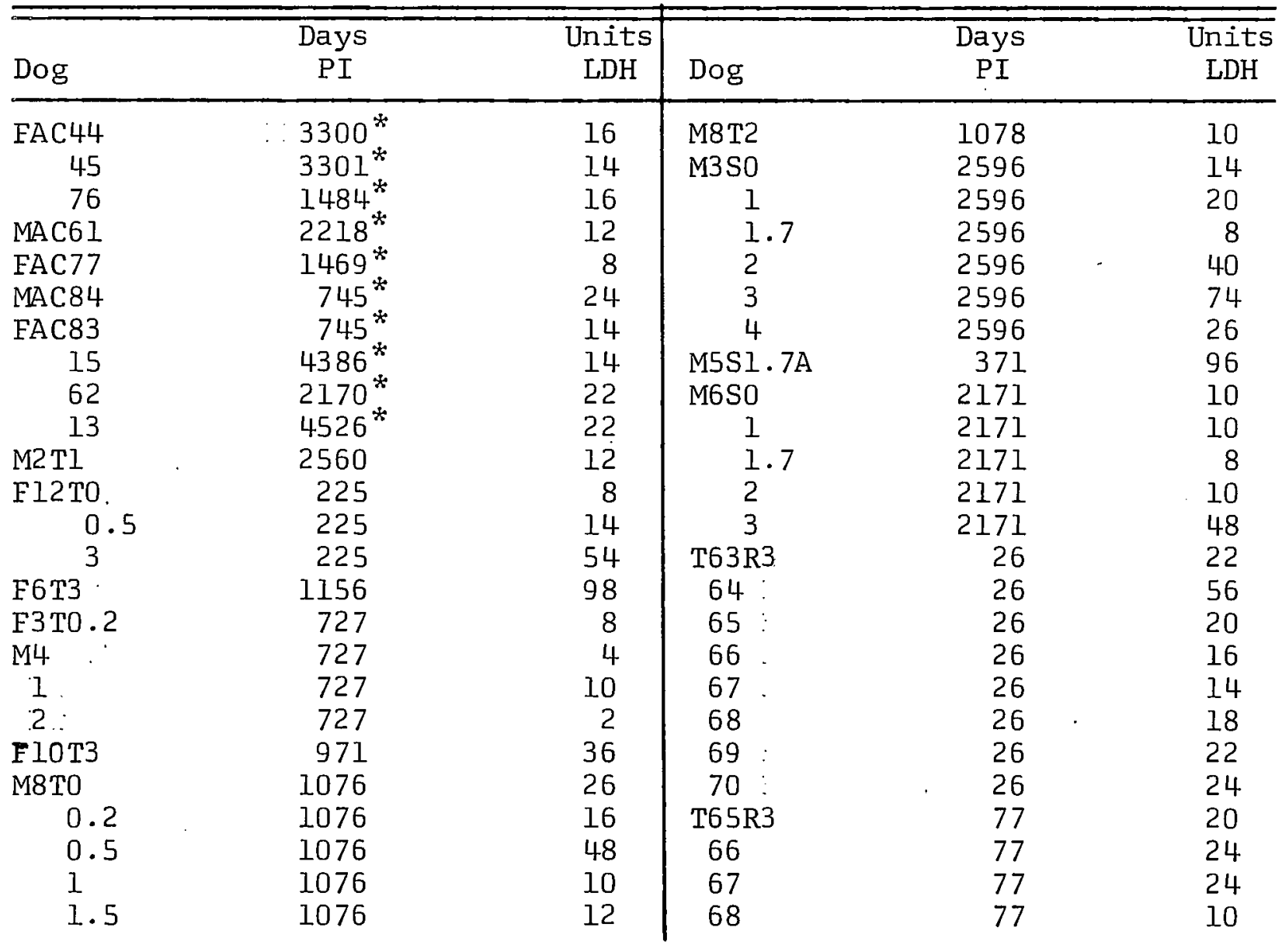

*Age at time of testing. 
(TABLE VI Con't.)

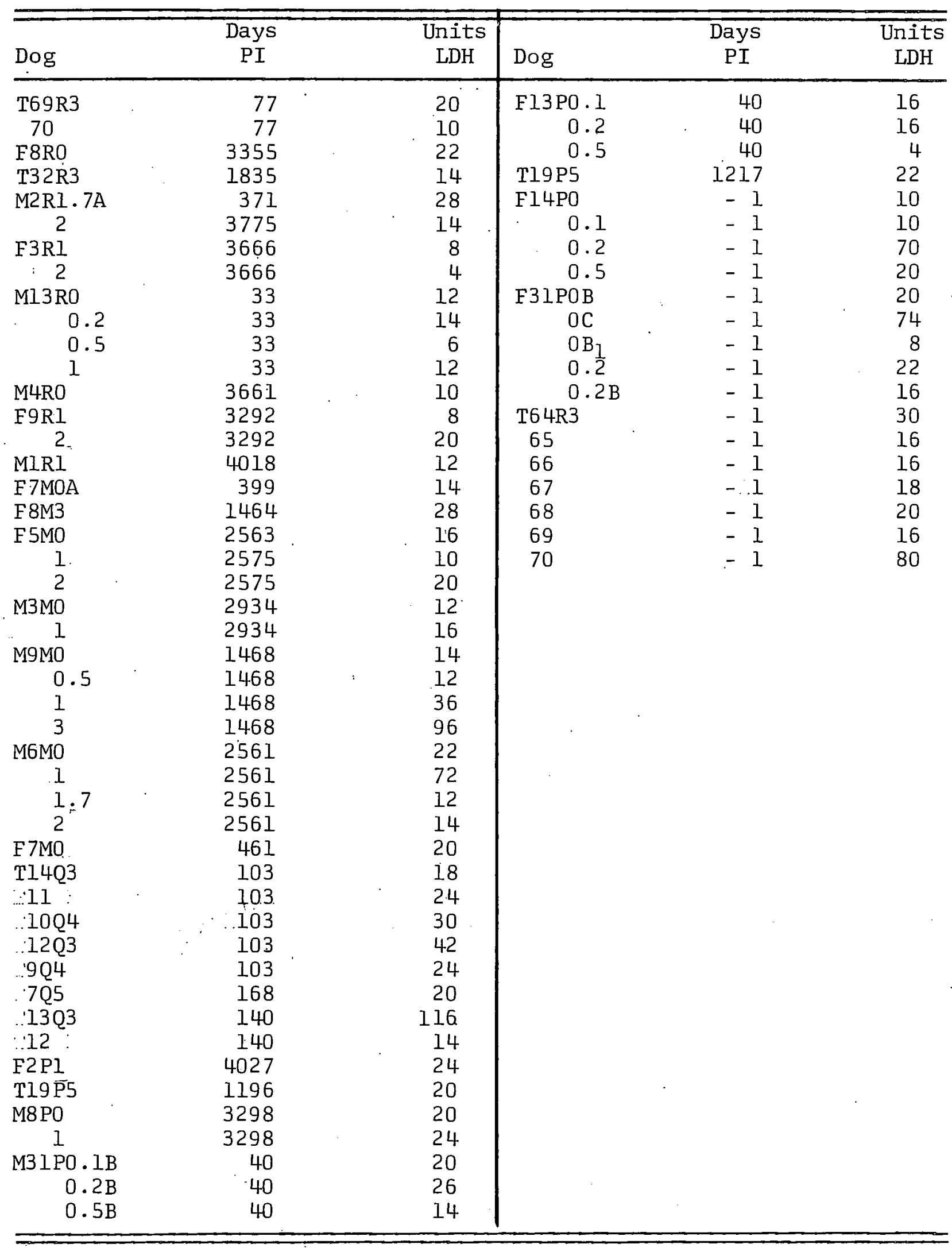


the analysis of data as a function of days elapsed since injection was considered as having possible pertinence. Although the data show that this factor is not of significance in this population, contending factors may have interacted, to obliterate a difference of LDH level with age. For example, the enzyme level in older dogs may diminish, while prolonged exposure to internally deposited radionuclides may give rise to conditions leading to elevated LDH. The latter supposition would tend to be discounted, however, on the basis of the analysis of the tumor bearing animals, since these did not show a statistically significant difference in enzyme level either. The dogs which were 3000 days or longer from injection did show a trend toward lower $\mathrm{LDH}$ values, with an average value 10 units below the next lower group. Comparison of youngest and oldest dogs confirmed that statistically, no difference exists, $(t=1.22, P=$ $0.2-0.3)$; nevertheless in view of the large standard deviations, the trend invites speculation.

The failure to discover any correlation between disease state and LDH level is interesting. Most studies, such as those of Bierman et al. (11), Zimmerman et al. (12), West and Zimmerman (4,13), Hsieh and Blumenthal (14), and Nishio et al. (15), present data as number of tests showing elevated LDH compared to total number of tests run. In all cases the percentages vary tremendously, and it is impossible to determine whether or not the differences are significant in any one experiment. In at least some of these, however $(12,13)$, there is illustrated some kind of correlation with malignancy, metastatic malignancy, or liver damage. The failure of this study to show any such correlation may be due to too small a sample siże. It is more likely, however, that lactic dehydrogenase 
activity in serum is not a reliable indicator of pathological states in the dog. Perusal of individual determinations shows several values over 100 units, in which no other abnormality could be found in the animal.

The data from the hemolysis studies indicate what could be logically deduced, namely that elevation of serum LDH level will occur if there is rupture of cells which contain the enzyme in large concentration (2). This, however, presents an interesting contrast with the work of Zimmerman et al. (16), who found normal LDH levels in patients suffering from hemolytic anemia. The mechanisms of hemolysis are, of course, markedly dissimilar, demonstrating that in vivo some metabolic process either removes the LDH from the serum or lowers its level in the weakened erythrocyte.

The main positive data to emerge from this study are the most puzzling. The fact that dogs receiving high levels of internal radiation have high serum LDH levels does not correlate with the normal LDH values found in animals afflicted with the diseases most commonly associated with high doses of the radionuclides. in question. Again, the paucity of data from tumor bearing and cirrhotic beagles may be the cause of the confusion. Large amounts of internally deposited radionuclide may either cause a metabolic change reflected in upswing of serum LDH activity or the elevated enzyme level may be indicating early, undetected pathologic states in animals which will eventually develop full blown disease. The nonspecificity of response of serum $\mathrm{LDH}$ level in disease states makes interpretation difficult.

\section{ACKNOWLEDGEMENTS}

The authors would like to thank Dr. B. J. Stover for her constructive criticisms, and Mr. F. W. Bruenger for his untiring help and expert 
advice regarding chemical proceedures and functioning of equipment. Dr. C. W. Mays' suggestions concerning statistical analyses were also greatly appreciated. 
1. Berger, L., and Broida, D., The colorimetric determination of lactic dehydrogenase in serum or other fluids at 400 to $550 \mathrm{m \mu}$. Sigma Chemical Company, St. Louis, Missouri (1960).

2. Wroblewski, F., and LaDue, J., Lactic dehydrogenase activity in blood. Proc. Soc. Exptl. Biol. Med. 90, 210-213 (1955).

3. Hsieh, K., Suntzeff, V., Cowdry, E., Serum lactic dehydrogenase activity as an indication of neoplastic growth and regression. Proc. Soc. Exp. Biol. Med. 89, 627-629 (1955).

4. West, M., Heller, P., Zimmerman, H., Serum enzymes in disease III Lactic dehydrogenase and glutamic oxaloacetic transaminase in patients with leukemia and lymphoma. Am. J. Med. Sci. 235, 689-701 (1959).

5. Snodgrass, P. J., Wacker, W. E., Eppinger, E. C., and Vallee, B. L., Metalloenzymes and myocardial infarction III Lactic dehydrogenase activity of serum-a determinate diagnostic measure. N.E.J.M. 261, $1259-1266$ (1959).

6. Vessell, E. S., and Bearn, A. G., The heterogeneity of lactic and malic dehydrogenase. N. Y. Acad. Sci. Ann. 75, 286-291 (1958).

7. Hill, B., Further studies of the fractionation of lactic dehydrogenase of blood. N. Y. Acad. Sci. Ann. 75:304-310 (1958).

8. Blanco, A., Zinkham, W. H., Lactic dehydrogenase in human testes. Science 139, 601-602 (1963).

9. Dougherty, T. F., Stover, B. J., Dougherty, J. H., Jee, W. S. S., Mays, C. W., Rehfeld, C. E., Christensen, W. R., and Goldthorpe, H. C. Studies of the biological effects of $\mathrm{Ra}^{226}$, Pu239, $\mathrm{Ra}^{228}$ (MsTh $)$, $\mathrm{Th}^{228}$ (RdTh), and $\mathrm{Sr}^{90}$ in adult beagles. Radiation Res. 17, 625681 (1962).

10. Dougherty, T. F., Research in Radiobiology. Annual report of work in progress on the chronic toxicity program. Radiobiology Division of the Department of Anatomy, U. of Utah College of Medicine, March 1964.

11. Bierman, H. R., Hill,, B., Emory, E., Reinhardt, L., and Samuels, A., Correlation of serum lactic dehydrogenase activity with olinical status of patients with neoplastic diseases. Proc. Am. Assoc. Cancer Res. 2, 5 (1955).

12. Zimmerman, H. J., Weinstein, H. C., Lactic dehydrogenase acticity in human serum. J. Lab. Clin. Med. 48, 607-616 (1956).

13. West, M., Zimmerman, H., Serum enzymes in disease I. Lactic dehydrogenase and glutamic oxalacetic transaminase in carcinoma. AMA Arch. Int. Med. 102, 103-114 (1958). 
14. Hsieh, K. M., Blementhal, H. T., Serum lactic dehydrogenase levels in various disease states. Proc. Soc. Exp. Biol. Med. 91, 626-630 (1956).

15. Nishio, K., Jacobson, K. B., Jenkins, V. K., and Upton, A. C., Studies on plasma lactic dehydrogenase in mice with myeloid leukemia I Relation of enzyme level to course of disease. Cancer Res. 23, 340-343 (1963) .

16. Zimmerman, H., West, M., Heller, P., Serum enzymes in disease II Lactic dehydrogenase and glutamic oxalacetic transaminase in anemia. AMA Arch. Int. Med. 102, 115-123 (1958). 
F. W. Bruenger, W. Stevens, B. J: Stover and D. L. Berliner

Abstract: The method of gel-filtration was used to study in vitro the binding and the distribution of Pu IV in dog plasma. It was found that the main portion of $\mathrm{Pu}$ was bound by albumin. Only a small amount of plutonium was associated with high molecular weight proteins. The medium weight globulins of type 7S and 4-5S were essentially free of plutonium.

Studies on the Pu content of plasma after intravenous injection of $\mathrm{Pu}^{239}$ have been published previously by this laboratory ${ }^{(1)}$. Approximately $85 \%$ of the injected $\mathrm{Pu}^{239}$ is circulating. in the plasma at one minute. Between one minute and ten.hours, the concentration dropped by a factor of about 2 and decreased from then until three weeks after injection by a factor of 250 . When compared to similar treatments using the alkaline earth elements in the place of $\mathrm{Pu}^{239}$, the plasma concentration of these elements decreased at a rate of more than ten times faster than that of plutonium ${ }^{(2)}$. Hence, the binding of $\mathrm{Pu}^{239}$ to the plasma constituents must be appreciably stronger.

The present investigations are an attempt to determine the binding sites of $\mathrm{Pu}$ in plasma and specifically to plasma proteins. All studies were carried out with in vitro tagged plasma proteins and isolated dog proteins.

Both heparinized and unheparinized blood from healthy dogs of the colony was incubated with Pu IV in the citrate buffer which is used for injection solutions. Plasma and bloö cells were separated by centrifugation. The blood cells were washed three times. with saline solution and analyzed for their Pu content. The uptake was very small, less than $0.5 \%$, evaluated for the amount of cells contained in $1 \mathrm{ml}$ of whole blood. 
These cells were ruptured and membranes separated from proteins by high speed centrifugation. The Pu was found to be associated with the cell membranes. No Pu had penetrated into the cells after one hour of incubation.

The plasma was subjected to ultrafiltration by the Toribara method (3). The ratio of the plutonium concentration in the clear ultrafiltrate to the concentration in the original plasma was only $6.774 \times 10^{-3} \pm 2.65$ $\times 10^{-3}$. No attempt, however, was made to make any prediction of the relative quantities of $\mathrm{Pu}$ associated with proteins and the relative quantity of $\mathrm{Pu}$ in ionic form since the cellophane bags (Visking Nojax casing) used were found to retain an appreciable quantity of $\mathrm{Pu}^{239}$ under the physiological conditions applied. The largest fraction of the activity, however, was bound by the non-filterable constituents.

In order to decide whether Pu was not taken up by the cellular fraction of the blood because of an unfavorable equilibrium produced by the fast uptake and tight binding of the metal ion by proteins, or be-' cause of the inability of plutonium to penetrate the membranes, blood cells were freed from plasma by repeated washings with either saline solution or a physiological phosphate buffer. The plasma contamination of the blood cells after four successive washings was less than one tenth of one percent. Cells, washed with saline solution, were incubated with Pu IV citrate for times of 0-24 hours, washed again with saline solution and analyzed for Pu. The uptake was very small (less than $1 \%$ ) and did not increase significantly with time. Cells washed with physiological phosphate buffer were incubated with Pu IV citrate for times of 0-96 hours. The Pu uptake in this case was higher and increased during 

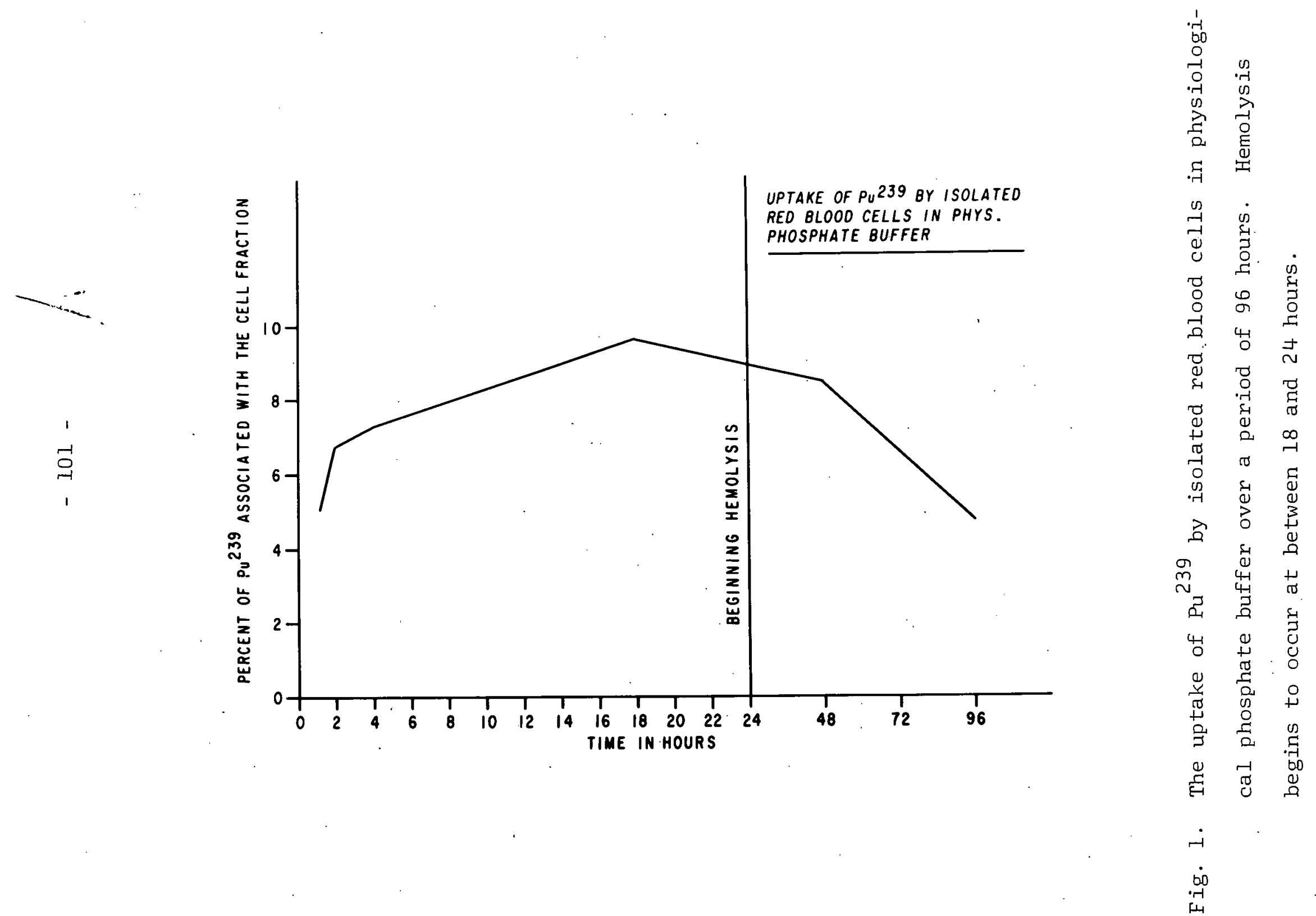


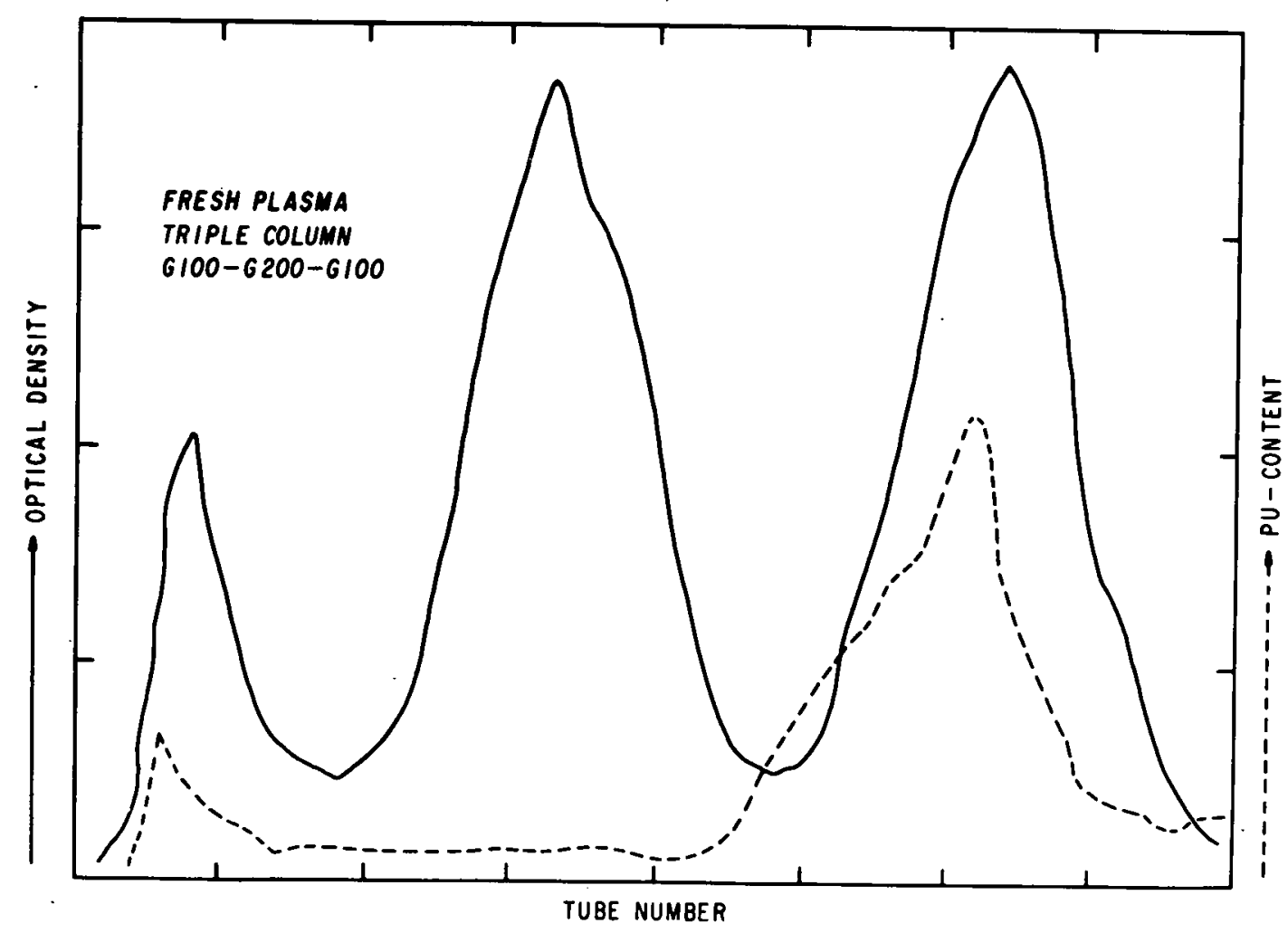

Fig. 2. Protein elution pattern (solid line) of fresh dog plasma after chromatography on Sephadex columns. The dotted line indicates the relative plutonium content of these proteins. 
the first 18 hours after incubation. The Pu content, however, declined during the time from 18-96 hours (Fig. 1). This decrease in activity was accompanied by a progressive hemolysis. As a result, it is found possibleto tag red blood cells with $\mathrm{Pu}^{239}$ if a proper buffer is chosen. Hemolysis, however, accompanied by a release of protein into the surrounding medium tends to reverse this reaction, which in turn is a sign of only weak bonding by the cells, possibly only the cell membranes.

Because of the high charge of the plutonium ion, Pu will detach from its protein-binding sites if a sufficient voltage gradient is applied. Therefore, electrophoretic methods have to be excluded from determinations of its binding sites as well as ion exchange chromatography. Gelfiltration methods, however, based primarily on molecular sieving, provided an excellent means for the separation of plasma proteins under mild conditions. Dextran gels of low cross linkage (Sephadex G-100 and G-200) were used in all experiments. The unit column size was 2 by $50 \mathrm{~cm}$. Whenever more than one unit was used, the columns were linked by small Teflon tubing. Fractions of approximately $4 \mathrm{ml}$ were collected in a Packard fraction collector Model 234. The protein content of these fractions was determined spectrophotometrically by reading in a Beckman D.U. spectrophotometer at a wave length of $280 \mathrm{m \mu}$, and the corresponding $\mathrm{Pu}$ concentration was measured by a liquid scintillation counting technique. A 4\% NaCl - 0.IM Tris solution of $\mathrm{pH} 7.5$ served as the elutriant throughout the experiment. Depending upon the cross linkage of the resin, fresh plasma was separated into $3 \mathrm{ma}$ in peaks. The protein composition of the different segments of each peak was determined electrophoretically. Peak one contained the fast moving macroglobulins and high molecular 
ש.

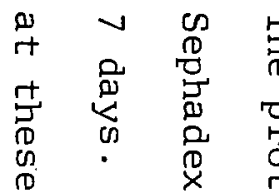

芦. 哥 :

元

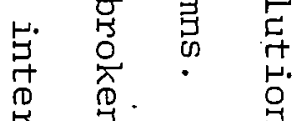

占. 点

恋

‥ 岁哥

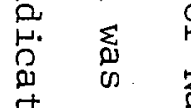

年 क 年

苛

12

त)

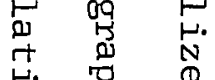

通畐 吕

च

w 0

○ i

皿号号

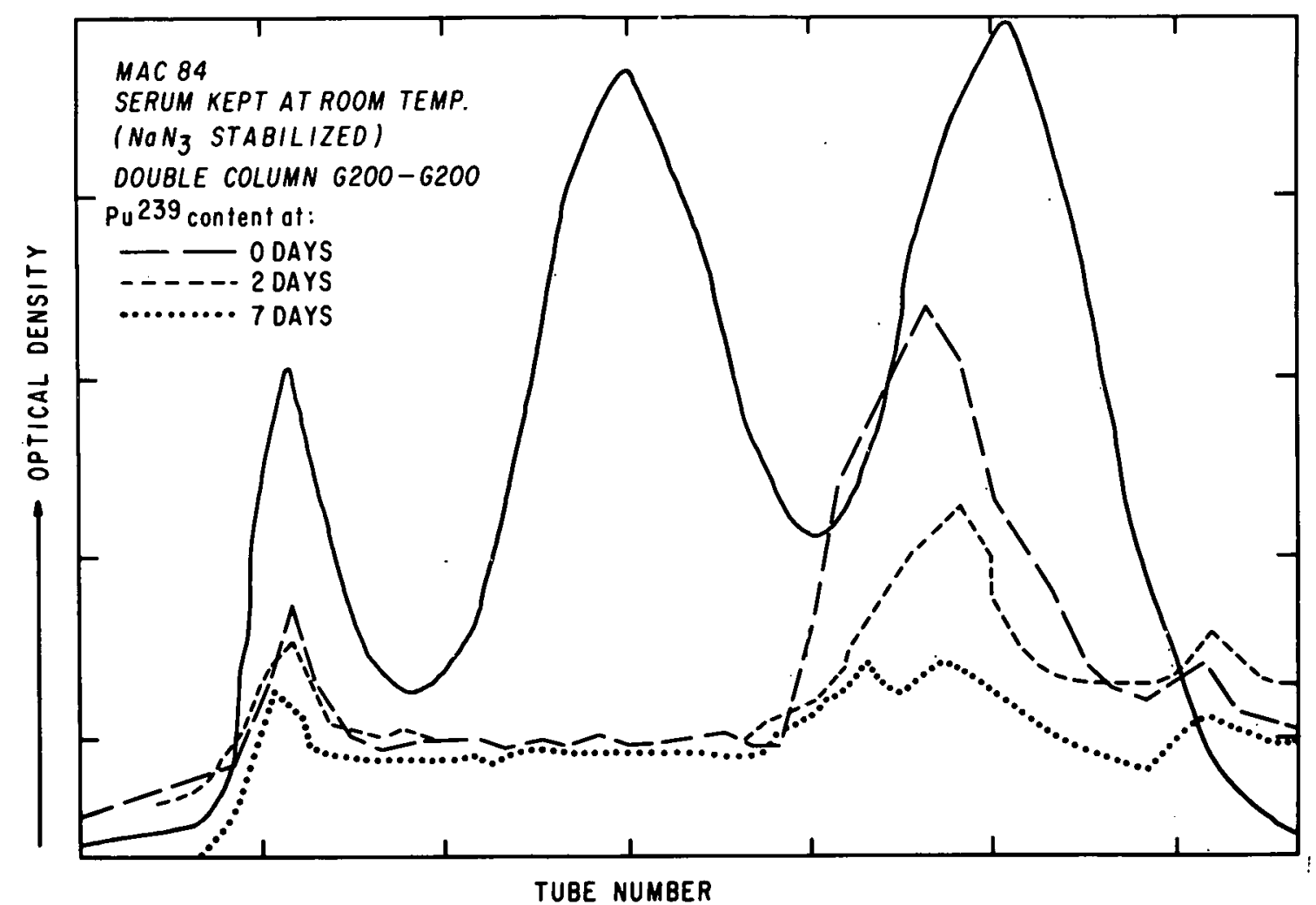

TUBE NUMBER 


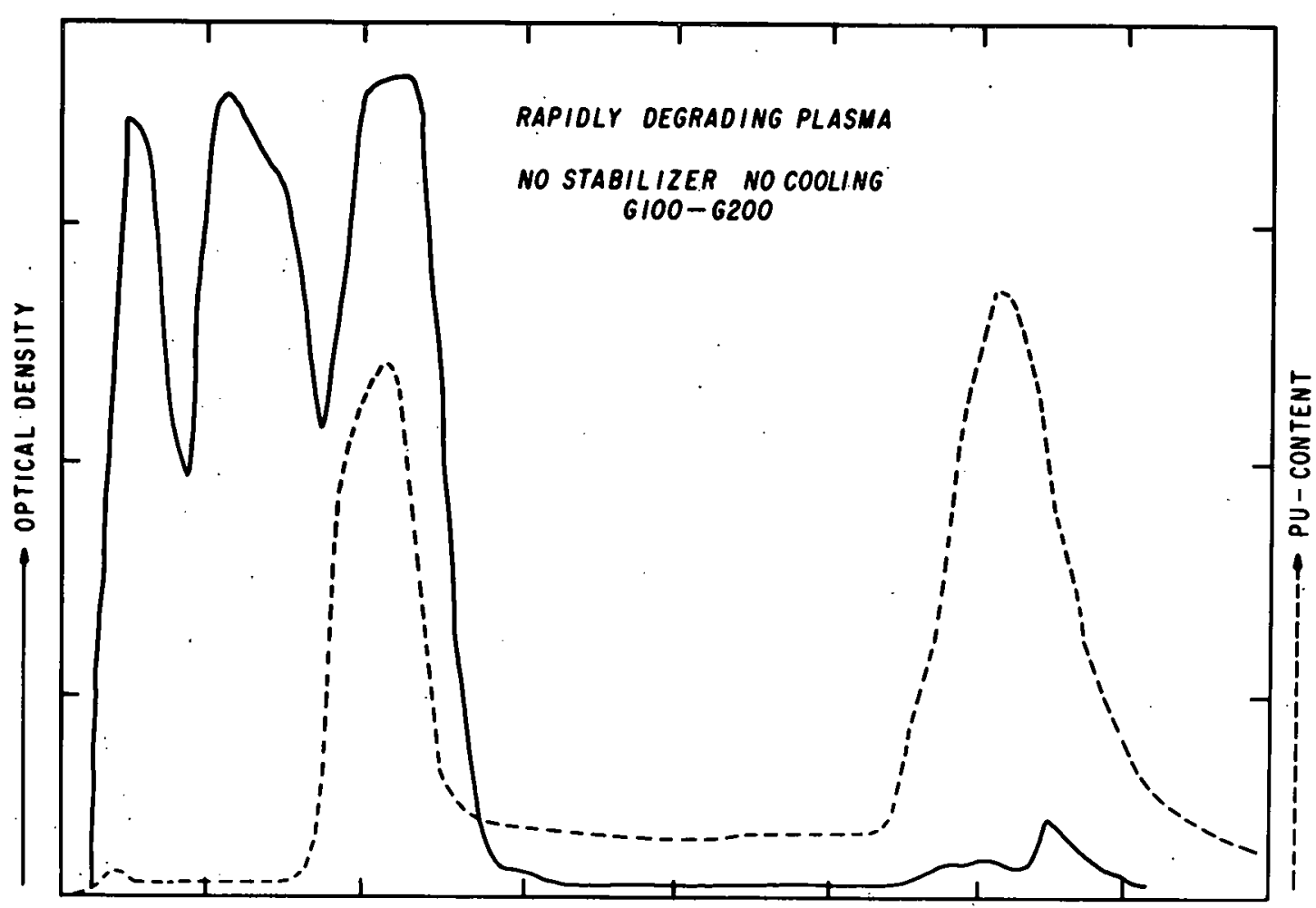

TUBE NUMBER

F-ig: 4. The protein elution pattern of rapidly degraded dog plasma from Sephadex columns. The broken line shows the relative $\mathrm{Pu}^{239}$ content associated with the protein peaks. 
weight lipoproteins (19S-type) . Results compare closely to those reported by Flodin (4). These were followed by globulins (7S and 4-5S-type). The third peak consisted mostly of albumin and some globulin (molecular weight from 66,000-150,000).

In order to get an idea of the relative binding affinities for the various proteins, dried bovine albumin and $\alpha, \beta, \gamma$-dog globulins were dissolved in physiological phosphate buffer, incubated with Pu IV citrate and subjected to gel filtration on a highly cross-linked gel (Sephadex G-25) which permitted the separation of protein bound Pu and nonprotein bound Pu. The $\alpha$ - and $\beta$ - globulins showed the highest attinity, tollowed by albumin and $\gamma$-globulin. No other meaning, however, should be attached to this experiment except that basically all proteins are capably of interacting with plutonium. The degree of hydration, the homogeneity, the spacing in the protein crystal, and the manner in which groups in side chains are surrounded by water dipoles and peptide linkages in the main chain are exposed, are certainly different in the living system. The distribution of Pu will depend to a high degree on those conditions and a certain selectivity is to: be expected.

Plasma was incubated for one hour with $\mathrm{Pu}^{239}$ and applied to a triple column (Sephadex G100-G200-G100). The corresponding spectra showed that some Pu was associated with the 195 type (high mol. wt.) proteins. Virtually no Pu was contained in the large second peak and the main portion of Pu appeared on the left shoulder of the third peak which contained largely albumin and some globulin. After a steep decline, the activity rose again slightly on the descending limb of the final protein peak (Fig. 2) 


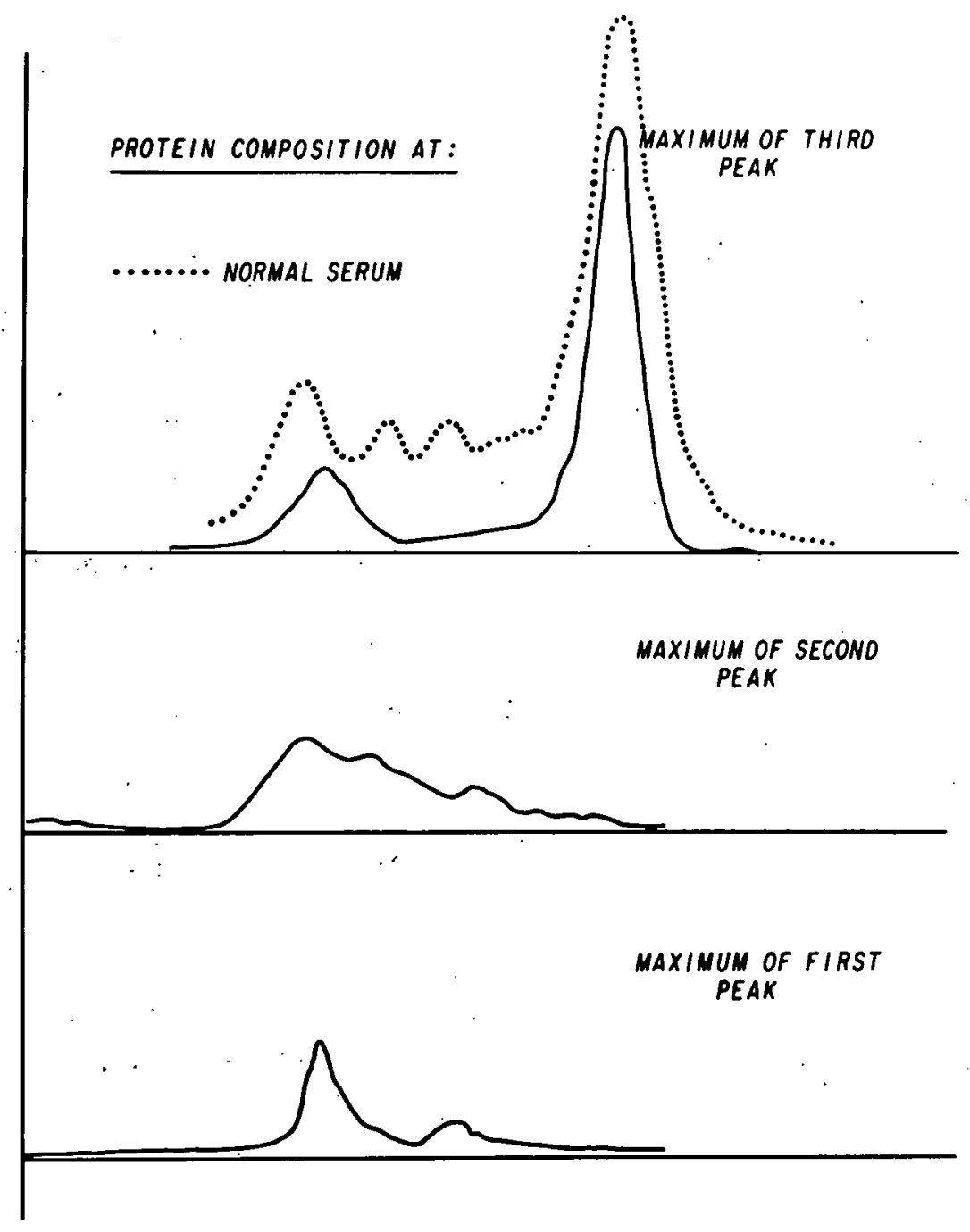

Fig. 5. Electrophoretic pattern of the proteins from each of the three peaks separated by gel filtration. The dotted line represents the spectrum of whole plasma. 
$\mathrm{Pu}^{239}$ tagged and $\mathrm{NaN}_{3}$ stabilized serum was chromatographed on a G200G200 column at the following times: 0, 2 days, 7 days. Although there was no significant change in the protein elution pattern, except for the appearance of a small fourth peak at a considerable distance to the right of the third peak, the plutonium content in peaks, number one and three became much smaller. The plutonium lost in these peaks appeared in the fourth peak which comprises low molecular weight peptides (Fig. 3).

Fig. 4 shows unstabilized and rapidly decomposing plasma of initially high $\mathrm{Pu}$ content. The activity to the right of :the third peak in this case did not decline to the usual low level but remained relatively high and ended in a strong peak at the region of low molecular weight peptides.

Fig. 5 illustrates the electrophoretic behavior of the proteins found in peaks one, two and three. The proteins of peak one are high molecular weight globulins (macroglobulins). The proteins of peak two are globulins $(\alpha, \beta \& \gamma)$ with a molecular weight of $1.5 \times 10^{5}-2.0 \times 10^{5}$. Peak three, which binds the largest amount of plutonium, was composed primarily of albumin MW 66,000 and a low molecular weight globulin, but it was not determined whether it was a $\beta$ or $\gamma$ globulin.

All of these experiments were. in vitro studies and not too much significance should be assigned to them in interpreting Iiving systems, but they provided the necessary experimental basis for subsequent in vivo investigation. For instance copper is at first bound by albumin and after a period of one day is almost quantitatively found in the ceruloplasm (5), so plutonium will probably redistribute itself also. Some kind of redistribution is to be expected from the change in the slope of the plasma concentration curve after one day post injection. The future investiga- 
tions will be an attempt to elucidate these changes in the living animal and possibly relate these changes to turnover times of the individual plasma protein constituents.

\section{REFERENCES}

1. B. J. Stover, D. R. Atherton and N. Keller, Rad. Res. 10; 130-147 (19.59) .

2. B. J. Stover; Health Physics $1,373-378$ (1959).

3. T. Y. Toribara, A. Raymond Terepka and Priscilla A. Dewey, The Journal of Clinical Investigation, XXXVI, 738-748 (1957).

4. P. Flodin and J. Killander, Biochimica and Biophysica Acta, 63, 403410 (1962).

5. Leon L. Milier, H. R. Hanavan, N. Tithasiri and A. Chowdhury, Amino and Serum Proteins, Advances in Chemistry Series 44, 17 (1964) ACSMonograph. 


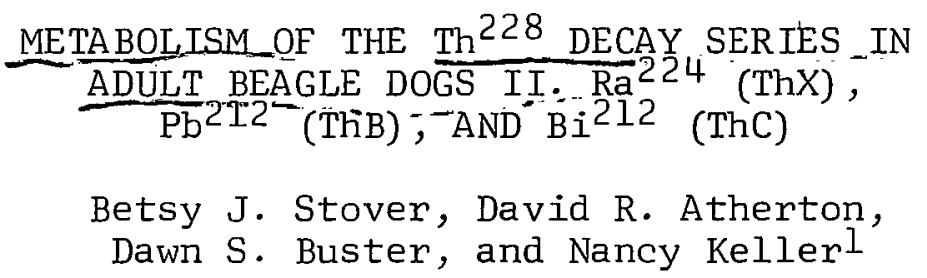

Abstract: Retention and distribution of $\mathrm{Ra}^{224}, \mathrm{~Pb}^{212}$, and $\mathrm{Bi}^{212}$ in beagles have been studied through three years after the intravenous injection of Th228, the parent nuclide, with the decay products in equilibrium. 'Initially $\mathrm{Ra}^{224}$ in plasma decreased more rapidly than $\mathrm{Th}^{2} 28$ in plasma. $\mathrm{Pb}^{2} 12$ was associated with blood cells. Later the concentrations of $\mathrm{Ra}^{224}$ in plasma and $\mathrm{Pb}^{212}$ in cells were respectively 24 and 480 times that of $\mathrm{Th}^{2} 28$ in plasma. Bi212 in plasma was about twice Ra224, and in cells it was about $0.9 \mathrm{~Pb}^{2} 12$.

At 30 days cumulative excretion of $\mathrm{Ra}^{224}$ was $35 \%$ compared with $11 \%$ for $\mathrm{Th}^{228} ; 27 \%$ of the $\mathrm{Ra}^{22} 24$ atoms formed in vivo were excreted. The $\mathrm{Ra}^{2} 24$ excretion rate decreased with time and at 100 and 1000 days, respectively, 12 and $7 \%$ of the atoms formed in vivo were excreted. The fecal excretion rate was greater than and decreased more slowly than the urinary rate: In the highest dose level studied $\mathrm{Ra}^{224}$ excretion rates decreased more rapidly than those of lower levels. At 777 days cumulative urinary excretion of $\mathrm{Pb}^{2} 12$ was $0.4 \%$, which was about $0.6 \%$ of the $\mathrm{Pb}^{212}$ atoms formed in vivo. Cumulative urinary excre-

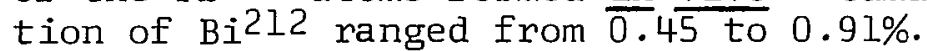

In the liver the activity of $\mathrm{Pb}^{2} 12$ was slightly higher than that of $\mathrm{Ra}^{2} 24$ which was about $30 \%$ higher than that of Th228. All 3 decrease at about the same rate, and this rate is about the same as observed for the 3 in blood. In the spleen $\mathrm{Ra}^{224}$ activity was again slightly higher than $\mathrm{Th}^{228}$ activity but $\mathrm{Pb}^{212}$ activity was only half that of Th228. The rates of decrease were about the same as for liver and blood.

In the kidneys it was also possible to measure $\mathrm{Bi}^{212}$. The mean $\mathrm{Bi}^{2} 12$ activity measured was $17 \%$ greater than that of Th228 which was 3.5 times that of $\mathrm{Ra}^{2} 24$ and $\mathrm{Pb}^{212}$, the means of the latter two being equal. There was considerable variation in all four sets of data and the rates of biological decrease could not be determined. Some of the Ra224 and Bi2l2 in kidneys and urine must come from extra-vascular sites. $\mathrm{Pb}^{212}$ in kidneys and urine comes mainly from $\operatorname{Tn}^{220}$ decay in blood and kidneys, but other extravascular sites are not excluded.

In one humerus and the third lumbar vertebra there was a progressive loss of decay products; the activity of $T^{2} 28$ was greater than that of $\mathrm{Ra}^{224}$ which exceeded $\mathrm{Pb}^{2} \mathrm{l} 2$.

1 Present address: Department of Physiology, Stanford University. 
Neither the $\mathrm{Ra}^{224}$ nor the $\mathrm{Pb} \mathrm{b}^{2} \mathrm{l}$-data showed a significant rate of biological decrease in either bone. Also the activities of $\mathrm{Ra} 224$ and of $\mathrm{Pb}^{212}$ relative to that of $\mathrm{Th}^{228}$ did not show:a significant change with time.

In summary, the biological rates of decrease of $\mathrm{Ra}^{224}$ and $\mathrm{Pb}^{2} 12$ were found to be about the same as those of Th228. The biological rate constants for the 3 nuclides in bone were found to be of the order of 0.1 of the physical decay constant. For blood, excreta, and soft tissues the biological rate constants were found to be the same order of magnitude as the physical decay constant. Average radiation dose rates to bone, liver, and kidneys have been. estimated, and the problem of evaluating the loci of decay of $\mathrm{Tn}^{220}$ and Po 216 has been discussed.

\section{INTRODUCTION}

The studies reported herein are a part of the long term studies of the biological effects of $\mathrm{Th}^{228}$ and other radionuclides in adult beagles (1). Previously we have reported the metabolism in these beagles of the parent nuclide, $\mathrm{Th}^{228}$, over a period of 3.5 years following injection (2) - Earlier, Van Dilla et al. (3) reported the translocation of $\operatorname{Ra}^{224}$ during the first few months after injection, and Mays et al. (4) reported the exhalation of $\operatorname{Tn}^{220}$ through 1000 days. In this communication we endeavor to complete the picture with a 3.5 year study of $\mathrm{Ra}^{224}, \mathrm{~Pb}^{2.12}$ and $\mathrm{Bi}^{212}$ in blood, excreta, and selected bones.and soft tissues. These. data with those for $\operatorname{Th}^{228}$ (2) and $\operatorname{Tn}^{220}$ (4) are then used to estimate average radiation dose rates to several tissues. METHODS

Each dog was given a single intravenous injection of $\operatorname{Th}^{228}$ with its decay products in equilibrium in young adulthood (I). The nearly carrier free $\operatorname{Th}^{228}$ injection solution has been described before (2) . $\mathrm{Ra}^{224}$ and $\mathrm{Pb}^{212}$ in the various blood, excreta, and tissue samples were determined by repeated counting in a well-type liquid scintillation gamma 
detector until equilibrium with $\mathrm{Th}^{228}$ was established. Then $\mathrm{Th}^{228}$ was measured in these same samples as has been reported (2) $\cdot \mathrm{Ra}^{224}$ and $\mathrm{Pb}^{212}$ activities were obtained by resolving the plots of count rate vs. time after sample collection or death. It was also possible by this method to determine $\mathrm{Bi}^{212}$ (ThC) in the kidneys and special urine samples. The exception to the above was the determination of $\mathrm{Ra}^{224}$ in plasma at times greater than 2 to 3 weeks after injection. By then the gamma activity was so low that alpha measurements were necessary. The plasma was ashed rapidly with nitric acid, and then an aliquot of the solution was placed on a stainless steel disc. Using a conventional $2 \pi$ proportional counter the alpha activity was measured repeatedly until equilibrium was established.

Determination of $\mathrm{Ra}^{224}, \mathrm{~Pb}^{212}$, and $\mathrm{Bi}^{212}$ as described above is valid only if the gammas detected can be identified with a specific nuclide (or nuclides). Gammas occur throughout the $\operatorname{Th}^{228}$ decay series. All gammas occurring prior to $\mathrm{Pb}^{212}$ are of low energy except one of $0.241 \mathrm{Mev}$ which occurs in $3.7 \%$ of the $\mathrm{Ra}^{224}$ disintegrations (5) . However, gammas of energy of $0.239 \mathrm{Mev}$ or $0.300 \mathrm{Mev}$ occur in about $77 \%$ of the $\mathrm{Pb}^{212}$ disintegrations. Thus it is sufficient to compare the detector response to $\mathrm{Pb}^{212}$ gammas with the response to the gammas from $\mathrm{Bi}^{212}$ and its decay products.

For this comparison $\mathrm{Pb}^{212}$ was obtained by bubbling air through a $\mathrm{Th}^{228}$ solution in a small container to carry $\mathrm{Tn}^{220}$ into a large flask. The flow rate and the volumes were chosen so that a large part of the $\operatorname{Tn}^{220}$ decayed in the flask. $\mathrm{Pb}^{212}$ was washed out and diluted to a known volume with $6 \mathrm{M} \mathrm{CH}_{3} \mathrm{COOH}$. Sufficient time was allowed for $\mathrm{Pb}^{212}$ and $\mathrm{Bi}^{212}$ 
to attain transient equilibrium. $\mathrm{Pb}^{212}$ and $\mathrm{Bi}^{212}$ were separated by precipitating $\mathrm{PbCrO}_{4}$; isotopic carriers were used for both nuclides. $\mathrm{PbCrO}_{4}$ was dissolved in $4 \mathrm{M} \mathrm{HNO}_{3}$.

Aliquots of the unseparated $\mathrm{Pb}^{212} \mathrm{Bi}^{212}$ solution and of the separated $\mathrm{Pb}^{212}$ and $\mathrm{Bi}^{212}$ solutions were counted serially in the liquid scintillator. The growth and decay of alpha activity in an aliquot of the $\mathrm{Pb}^{212}$ was also measured. The three decay curves were then resolved.

By comparison with the unseparated sample, loss during the separation was negligible; the fractional recovery was $0.99 \pm 0.03$. The degree of separation was determined from the components of the alpha curve and the 10.6 hour component of the gamma curve for the $\mathrm{Bi}^{212}$ solution. The fraction of $\mathrm{Pb}^{212}$ left in the $\mathrm{Bi}^{212}$ solution was only 0.02 while the fraction of $\mathrm{Bi}^{212}$ carried with the $\mathrm{PbCrO}_{4}$ was 0.19 . These results are consistent with the fact no special procedures, suchas reprecipitation, were done since the short $\mathrm{Bi}^{212}$ half-period imposed a time limit. From these yields and the remaining gamma curve components it was determined that the efficiency of the detector for $\mathrm{Pb}^{212}$ gammas was not significant with respect to ( $0-1 \%$ of) that for the gammas from $B i^{212}$ and decay products. Without this important result the growth and decay data obtained from samples from dogs given $T^{228}$ could not be meaningfully analyzed. Blood cells and plasma were separated by centrifugation and then counted without further treatment, except for the late plasmas described above. Continuous excretion studies were made for the first 22 days after injection for two dogs and 30 days for two dogs. The urine and feces were ashed as rapidly as possible, and then $: 50 \mathrm{ml}$ aliquots were counted serially to determine $\mathrm{Ra}^{224}$. Later 96 hour collections were 
made. An aliquot of the urine was sealed in a No. 2 can. A little distilled water was added to the feces, then they were homogenized in a blender, and an aliquot was sealed in a can. Phenol was used as a preservative in both cases. $\mathrm{Ra}^{224}$ was then determined by counting in a larger but very similar weil-type counter.

The samples obtained at autopsy were the left humerus, the third lumbar vertebra, one kidney, and known fractions of the liver and spleen. These samples were put in $50 \mathrm{ml}$ centrifuge tubes, covered with formalin, and sealed. The serial counting was begun one to three frours after death of the dog and continued until equilibrium was evident. RESULTS AND DISCUSSION

In presenting the results, the activity of $\mathrm{Ra}^{224}$ in a specific sample is given as the percent of the $\mathrm{Ra}^{224}$ activity which would be in equilibrium with that amount of $\mathrm{Th}^{228}$ which had been injected and allowed to decay to the time of sample collection. Percent $\mathrm{Ra}^{224}$ is defined by Eq. (1) and is

$$
\% \mathrm{Ra}^{224}=\frac{\mu \mathrm{C} \mathrm{Ra}^{224} \text { in sample } \times 100}{\mu \mathrm{C} \mathrm{Th}^{228} \text { injected } \times \mathrm{e}^{-\lambda} \mathrm{Th}^{228} \mathrm{t}}
$$

Percent $\mathrm{Pb}^{212}$ and percent $\mathrm{Bi}^{212}$ are defined in the same way. These definitions separate biological and radioactive decay factors and also permit direct comparison of the activities of the decay products with that of the series parent, $\mathrm{Th}^{228}$. The $\mathrm{Th}^{228}$ data were present similarly and in reference (2) 


$$
\begin{gathered}
\% \operatorname{Th}^{228}=\% \cdot \operatorname{Th}^{228} \text { injected } \times e^{\lambda} \operatorname{Th}^{228} t \\
=\frac{\mu c \operatorname{Th}^{228} \text { in sample } x \cdot 100}{\mu c \operatorname{Th}^{228} \text { injected } x e^{-\lambda} \operatorname{Th}^{228 t}}
\end{gathered}
$$

Thus, if the ratio of $\mathrm{Ra}^{224}$ activity to $\mathrm{Th}^{228}$. activity is desired at $t=$ T, Eqs.. (1): and (2): yield

$$
\frac{\% \mathrm{Ra}^{224}(\mathrm{~T})}{\% \mathrm{Th}^{228}(\mathrm{~T})}=\frac{\mu \mathrm{c} \mathrm{Ra}^{224} \text { in sample }}{\mu \mathrm{C} \mathrm{Th}^{228} \text { in sample }}
$$

Analogous comparisons of $\mathrm{Pb}^{212}$ and $\mathrm{Bi}^{212}$ activities relative to that of $\mathrm{Th}^{228}$ can be made.

Single exponentials, $y=a e^{-b t}$ were calculated for the various sets of data by applying. the classical least squares method to the transformed equation $\log y=\log : a-b t \log e$. The $90 \%$ symmetrical confidence limits for the parameters in the equations which follow are given in Table I. The t-test was used to determine whether b differed significantly from zero, and the resulting value of $p$ is given in the last column of Table I. The single exponential was chosen. arbitrarily to facilitate dose rate calculations which include the exponential decay factor.

Blood. The concentrations of $\mathrm{Ra}^{224}$ in blood plasma and of $\mathrm{Pb}^{212}$. in blood cells were measured through 1234 days after injection of $\mathrm{Th}^{228}$ in equilibrium with its decay products. From 2 minutes through 64 days blood from four 5 -level $\left(2.8 \mu \mathrm{c} \mathrm{Th}{ }^{228} / \mathrm{kg}\right.$ injected) and two 4-level (0.90 $\mu \mathrm{c} / \mathrm{kg}$ ) dogs was measured. The average and the range of $\% \mathrm{Ra}^{224} / \mathrm{g}$. plasma 
and of $\% \mathrm{~Pb}^{212} / \mathrm{g}$ cells in the 6 dogs are shown in Figs. 1 and 2, respectively. The measurements which were made from 77 to 1234 days are also

TABLE I

Equation Constants ${ }^{\mathrm{a}}$ with $90 \%$ Symmetrical Confidence Limits

\begin{tabular}{|c|c|c|c|c|c|c|c|}
\hline Eq. No. & & $\begin{array}{r}\text { Quanti } \\
\text { Nucl }\end{array}$ & and & $n$ & $\log a$ & $b \times 10^{3}$ & $\mathrm{p}$ \\
\hline 4 & $\mathrm{P}$ & & $\mathrm{Ra}^{224}$ & 31 & $-3.253 \pm 0.078$ & $1.31 \pm 0.34$ & $<0.01$ \\
\hline 6 & $\mathrm{C}$ & & $\mathrm{Pb}^{212}$ & 32 & $-1.958 \pm 0.088$ & $0.97 \pm 0.21$ & $<0.01$ \\
\hline 17 & $\mathrm{U}$ & (all) & $\mathrm{Ra}^{224}$ & 67 & $-0.184 \pm 0.071$ & $0.92 \pm 0.26$ & $<0.01$ \\
\hline 18 & $\mathrm{U}$ & (no 4) & $\mathrm{Ra}^{224}$ & 53 & $-0.130 \pm 0.056$ & $0.91 \pm 0.19$ & $<0.01$ \\
\hline 19 & $\mathrm{U}$ & (4) & $\mathrm{Ra}^{224}$ & 14 & $-0.194 \pm 0.122$ & $1.91 \pm 0.53$ & $<0.01$ \\
\hline 20 & $\mathrm{~F}$ & (all) & $\mathrm{Ra}^{224}$ & 65 & $+0.056 \pm 0.114$ & $0.53 \pm 0.15$ & $<0.01$ \\
\hline 21 & $\mathrm{~F}$ & (no 4) & $\operatorname{Ra}^{224}$ & 51 & $+0.062 \pm 0.062$ & $0.48 \pm 0.15$ & $<0.01$ \\
\hline 22 & $F$ & (4) & $\mathrm{Ra}^{224}$ & 14 & $+0.135 \pm 0.098$ & $1.24 \pm 0.42$ & $<0.01$ \\
\hline 23 & $X$ & (all) & $\mathrm{Ra}^{224}$ & 65 & $+0.266 \pm 0.068$ & $0.75 \pm 0.24$ & $<0.01$ \\
\hline 24 & $X$ & (no 4) & $\mathrm{Ra}^{224}$ & 51 & $+0.287 \pm 0.076$ & $0.72 \pm 0.26$ & $<0.01$ \\
\hline 25 & $\mathrm{X}$ & (4) & $\mathrm{Ra}^{224}$ & 14 & $+0.305 \pm 0.079$ & $1.43 \pm 0.34$ & $<0.01$ \\
\hline 49 & L & & $\mathrm{Ra}^{224}$ & 14 & $+0.719 \pm 0.125$ & $1.05 \pm 0.43$ & $<0.01$ \\
\hline 51 & L & & $\mathrm{Pb}^{212}$ & 14 & $+0.736 \pm 0.180$ & $1.03 \pm 0.63$ & $<0.02$ \\
\hline 52 & $\mathrm{~S}$ & & $\mathrm{Ra}^{224}$ & 11 & $-0.470 \pm 0.184$ & $1.10 \pm 0.73$ & $<0.05$ \\
\hline 54 & $\mathrm{~S}$ & & $\mathrm{~Pb}^{212}$ & 11 & $-0.800 \pm 0.223$ & $0.91 \pm 0.88$ & $<0.10$ \\
\hline
\end{tabular}

${ }^{a}$ Calculated by classical method of least squares.

shown in Figs. 1 and 2 where each point represents a single measurement. 


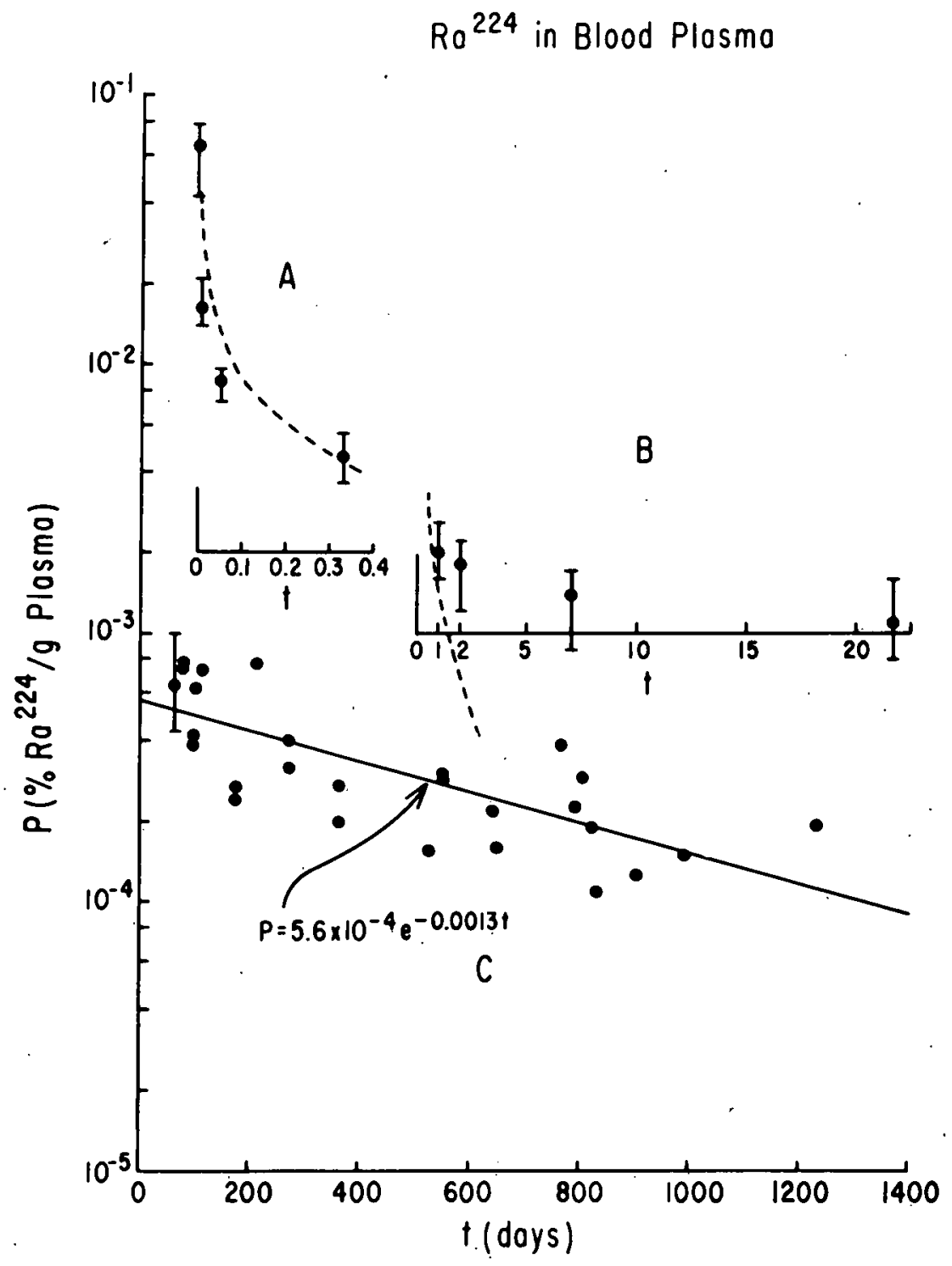

Fig. 1. \% $\mathrm{Ra}^{224} / \mathrm{g}$ plasma. Dotted curves in sections $\mathrm{A}$ and $\mathrm{B}$ show expected contributions of injected $\mathrm{Ra}^{224}$. 


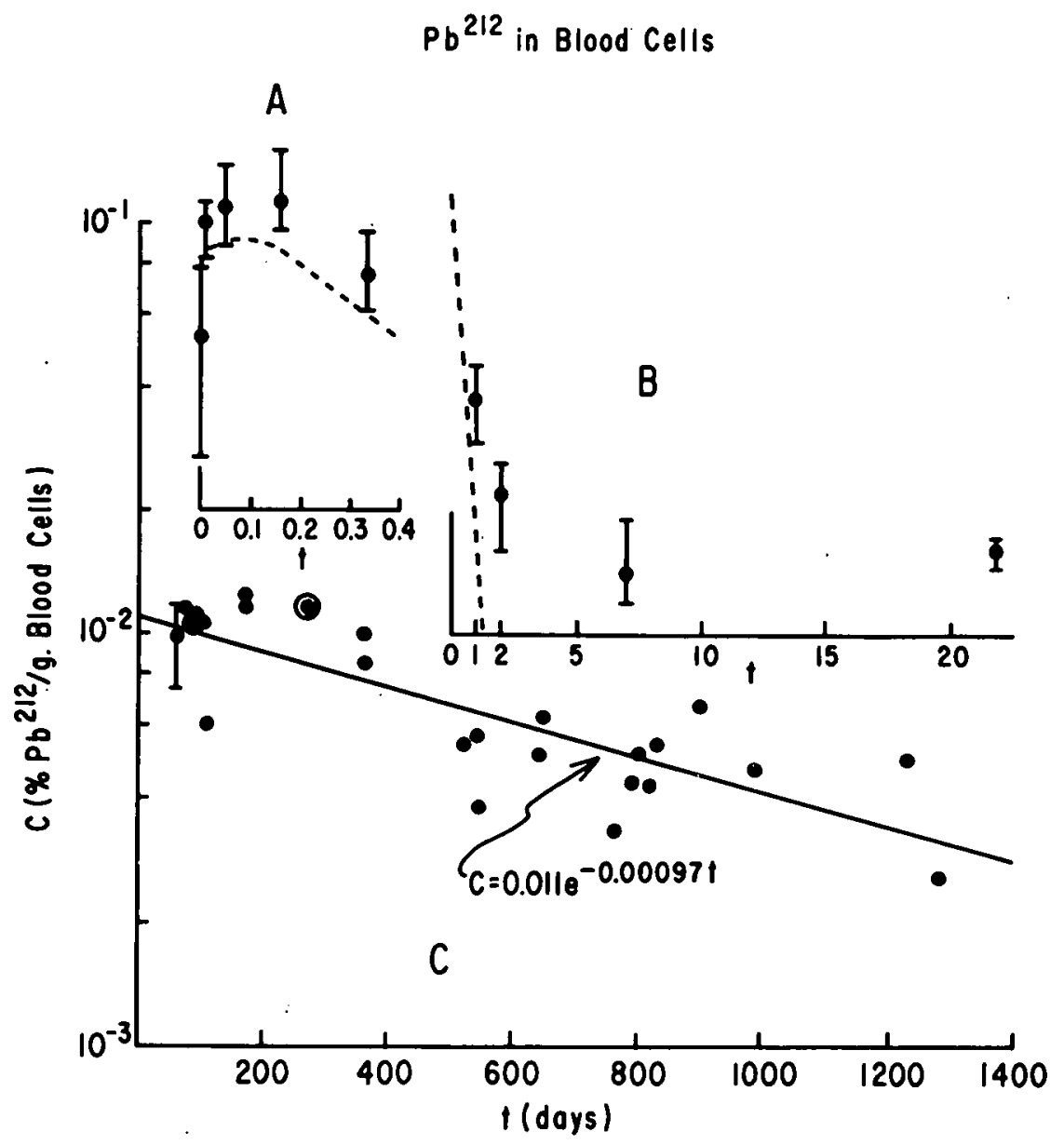

Fig. 2. $\% \mathrm{~Pb}^{212} / \mathrm{g}$ blood cells. Dotted curves in sections $\mathrm{A}$ and $\mathrm{B}$ show - expected contribution of injected $\mathrm{Pb}^{212}$. 
The samples were obtained mainly from. $3-(0.30 \mu \mathrm{c} / \mathrm{kg})$ and $4-$ level dogs with a few from $2-(0.096 . \mu \mathrm{c} / \mathrm{kg})$ and 5-level dogs, and many samples were obtained shortly before euthanasia.

In blood $\operatorname{Th}^{228}$ is essentially all in the plasma in non-diffusible form. From 2 minutes to 4 hours the concentration decreases by a factor of 3 . At 3 weeks the concentration is only $10^{-4}$ of that at 2 minutes. Thereafter concentration decreases at a slower rate. Initially $\mathrm{Ra}^{224}$ in plasma.drops. much more rapidly. At 2 and 15 minutes the $\mathrm{Ra}^{224}$ to $\mathrm{Th}^{228}$ concentration ratios are about 0.4 and 0.1 , respectively. At 72 minutes the ratio. for 2 dogs remained near 0.1 but was too low to measure for the other 4. This was the case for all 6 dogs at 4 hours, and for 3 of them at 8 hours when the other 3 averaged nearly 0.2 . The $\operatorname{Ra}^{224}$ to $\mathrm{Th}^{228}$ ratio then increases as the injected $\mathrm{Ra}^{224}$ disappears and $\mathrm{Ra}^{224}$ formed in vivo predominates. Values for 1 day and 1 week are 0.5 and:11, respectively. The dotted curve in Fig. I shows how the concentration of injected $\mathrm{Ra}^{224}$ would decrease. It was calculated from data for $\mathrm{Ra}^{226}$ in beagle plasma (6) to which appropriate $\mathrm{Ra}^{224}$ decay factors had been applied.

The data for \% $\mathrm{Ra}^{224} / \mathrm{g}$ plasma from 63 to 1234 days can be described by a single exponential which is

$$
P\left(\mathrm{Ra}^{224}\right)=5.6 \times 10^{-4} \mathrm{e}^{-0.0013 t}
$$

For this period $\mathrm{P}\left(\mathrm{Th}^{228}\right)$ shows the same time dependence (2) - Thus, 


$$
\frac{P\left(\mathrm{Ra}^{224}\right)}{P\left(\mathrm{Th}^{228}\right)}=\frac{5.6 \times 10^{-4} \mathrm{e}^{-0.0013 t}}{2.3 \times 10^{-5} \mathrm{e}^{-0.0013 t}} \cong 24
$$

and average $\mathrm{Ra}^{224}$ activity in plasma is about 24 times that of $\mathrm{Th}^{228}$.

In beagle blood $\mathrm{Pb}^{212}$ is predominantly (>98\%) in the cellular fraction (7). In these studies determination of $\mathrm{Pb}^{212}$ in plasma and $\mathrm{Ra}^{224}$ in cells was not feasible, either because of the predominance of the other nuclide or because of ashing of some plasmas.

At first $\mathrm{Pb}^{212}$ in blood decreased rapidly and at 2 minutes the average concentration was about the same as that of $\mathrm{Ra}^{224}$. Very soon some of the $\mathrm{Pb}^{212}$ returned to the blood and the peak concentration was more than twice the two minute value. The dotted line in Fig. 2 shows how injected $\mathrm{Pb}^{212}$ would decrease (7) - The peak was reached shortly after 15 minutes and persisted through 4 hours (Fig. 2) at which time the concentration of $\mathrm{Pb}^{212}$ in cells was about twice that of $\mathrm{Th}^{228}$ in plasma. Thereafter, the activity of $\mathrm{Pb}^{212}$ in blood remained greater than that of $\mathrm{Ra}^{224}$ or $\mathrm{Th}^{228}$. The data for $\% \mathrm{~Pb}^{212} / \mathrm{g}$ cells from 63 to 1282 days have been described by a single exponential which is

$$
\mathrm{C}\left(\mathrm{Pb}^{212}\right)=0.0110 \mathrm{e}^{-0.00097 t}
$$

The coefficients of $t$ in Eqs. (4) and (6) do not differ significantly, by the t-test. Thus, within the limits of the measurements, the concentrations of $\mathrm{Pb}^{212}$ in cells and $\mathrm{Ra}^{224}$ and $\mathrm{Th}^{228}$ in plasma decrease at the same rate. From 2 months to 3.5 years the following relation holds: 


$$
\mathrm{C}\left(\mathrm{Pb}^{212}\right) \cong 20 \mathrm{P}\left(\mathrm{Ra}^{224}\right) \cong 480 \mathrm{P}\left(\mathrm{Th}^{228}\right)
$$

Measurement of $\mathrm{Bi}^{212}$ in plasma and cells was difficult since centrifugation and separation required an appreciable part of a $\mathrm{Bi}^{212}$ halfperiod. However, the concentration of $\mathrm{Bi}^{212}$ in plasma was measured at 15 times ranging from:a few minutes to 1 year after injection. The results are given in Table II which also includes the $\mathrm{Ra}^{224}$.concentration

TABLE II

Activity of $\mathrm{Bi}^{212}$ in Plasma Compared
with that. of $\mathrm{Ra}^{224}$

\begin{tabular}{|c|c|c|c|c|}
\hline Dog & (days) & $\% \mathrm{Bi}^{212} / \mathrm{g}$ & $\% \mathrm{Ra}^{224} / \mathrm{g}$ & $\mathrm{Bi}^{212} / \mathrm{Ra}^{224}$ \\
\hline T4T5 & 0.0016 & 0.051 & 0.063 & 0.8 \\
\hline T5T5 & 0.0026 & 0.030 & 0.042 & 0.7 \\
\hline T5T5 & 0.0104 & 0.026 & 0.0072 & 3.6 \\
\hline T5T5 & 0.0513 & 0.004 & $<0.004$ & $>1.0$ \\
\hline T4T5 & 1.00 & $1.0 \times 10^{-3}$ & $2.4 \times 10^{-3}$ & 0.5 \\
\hline T4T5 & 2.00 & $1.2 \times 10^{-3}$ & $2.0 \times 10^{-3}$ & 0.6 \\
\hline T5T5 & 6.99 & $1.0 \times 10^{-3}$ & $1.0 \times 10^{-3}$ & 1.0 \\
\hline T5T5 & 21 & $1.3 \times .10^{-3}$ & $0.8 \times 10^{-3}$ & 1.6 \\
\hline $.74 T 5$ & 21 & $1.7 \times 10^{-3}$ & $0.9 \times 10^{-3}$ & 1.9 \\
\hline T4T5 & 63 & $0.93 \times 10^{-3}$ & $0.49 \times 10^{-3}$ & 1.9 \\
\hline T5T5 & 65 & $1.1 \times 10^{-3}$ & $0.51 \times 10^{-3}$ & 2.2 \\
\hline M2T5 & 97 & $0.92 \times 10^{-3}$ & $0.63 \times 10^{-3}$ & 1.5 \\
\hline $\mathrm{M} 2 \mathrm{~T} 4$ & 105 & $1.0 \times 10^{-3}$ & $0.4 \times 10^{-3}$ & 2.5 \\
\hline T4T5 & 113 & $0.67 \times 10^{-3}$ & $0.74 \times 10^{-3}$ & 0.9 \\
\hline M1T4 & 364 & $1.0 \times 10^{-3}$ & $0.6 \times .10^{-3}$ & 1.7 \\
\hline
\end{tabular}


in the same samples. From 21 days to 1 year the average $\mathrm{Bi}^{212}$ to $\mathrm{Ra}^{224}$ ratio is 1.8 . Thus we estimate the long term plasma contration of $\mathrm{Bi}^{212}$ as

$$
\mathrm{P}\left(\mathrm{Bi}^{212}\right) \sim 2 \mathrm{P}\left(\mathrm{Ra}^{224}\right) \sim 0.1 \mathrm{C}\left(\mathrm{Pb}^{212}\right)
$$

The source of the plasma $\mathrm{Bi}^{212}$ could be either the blood cells or extravascular sites or both. Early counts obtained on the cell samples did not yield a definitive answer. In connection with this problem it should be noted that there will always be an early increase in the count rate, i.e. $\mathrm{Bi}^{212}$ activity, in these cell samples as soon as $\mathrm{dC} / \mathrm{dt}$. becomes small compared with the $\mathrm{Pb}^{212}$ decay rate. The reason is that, when $\mathrm{Pb}^{212}$ is at a steady state in the blood, the activities of $\mathrm{Pb}^{212}$ and $\mathrm{Bi}^{212}$ will be related by conditions of secular equilibrium. When the blood is removed from the dog the steady state no longer exists and $\mathrm{Pb}^{212}$ disappears according to its decay constant, and $\mathrm{Bi}^{212}$ activity increases until transient equilibrium conditions are attained. Then, using $A$ to denote activity, we have

$$
{ }_{\mathrm{B} i}{ }^{212}=\frac{\lambda_{\mathrm{Bi}^{212}}}{\lambda_{\mathrm{Bi}}{ }^{212}-\lambda_{\mathrm{Pb}^{212}}} \quad{ }_{\mathrm{Pb}^{2}}{ }^{212}=1.11 \mathrm{~A}_{\mathrm{Pb}}{ }^{212}
$$

Thus, if $\mathrm{Bi}^{212}$ in plasma came from cells, the anticipated increase in cell count rates would be small and of about the same magnitude is the increase resulting from a change from secular to transient equilibrium conditions. 
It was usually 30 to 40 minutes after the blood was drawn when the first count was made, and usually an early increase was noted. However, the data obtained sufficed only to show that most of the $\mathrm{Bi}^{212}$ formed in the cells also decays there. Thus the blood cells are a mobile alpha source and irradiate capillary walls and other cells which may come in range.

Excretion.. The results of the early continuous excretion studies are shown in Figs. 3 and 4 in which the means and ranges are plotted. The injected doses for the dogs studied 22 days were 2.59 and $2.32 \mu \mathrm{c} /$ kg, (5-level) and those for the 30 day study were 0.884 (4-level) and 0.298 (3-level) $\mu \mathrm{c} / \mathrm{kg}$. The dogs given the two highest doses exhibited fever, loss of appetite, etc., as early as 9 days ( 1 ), and this appears to have affected fecal excretion of $\mathrm{Ra}^{224}$.

The cumulative urinary excretion rose to an apparent steady state value of $15 \%$ with a range of only $2-3 \%$. (Fig. 3). Much greater variation was seen in the cumulative fecal excretion (Fig. 3). It rose rapidly to about $20 \%$, then at about 10 days the two 5 -level dogs began to show a decrease which accounts for the dip in mean values from 12-22 days. Urinary plus fecal excretion (Curve A, Fig. 4) has a similar dip. (Note that cumulative excretion can decrease when the daily excretion of $\mathrm{Ra}^{224}$ is less than the daily decrease by decay of the total $\mathrm{Ra}^{224}$ outside the dog) - Curve $B$ is the mean cumulative excretion of $\mathrm{Th}^{228}$ by the same dogs over the same intervals. For comparison, the ranges for 10 and 22 days are shown. By 10 days the $\mathrm{Th}^{228}$ excretion rate was fairly low and there was no important effect on cumulative $\mathrm{Th}^{228}$ excretion from. 10 to. 22 days for the 5-level dogs. Curve $\mathrm{C}$ shows the cumulative excretion 


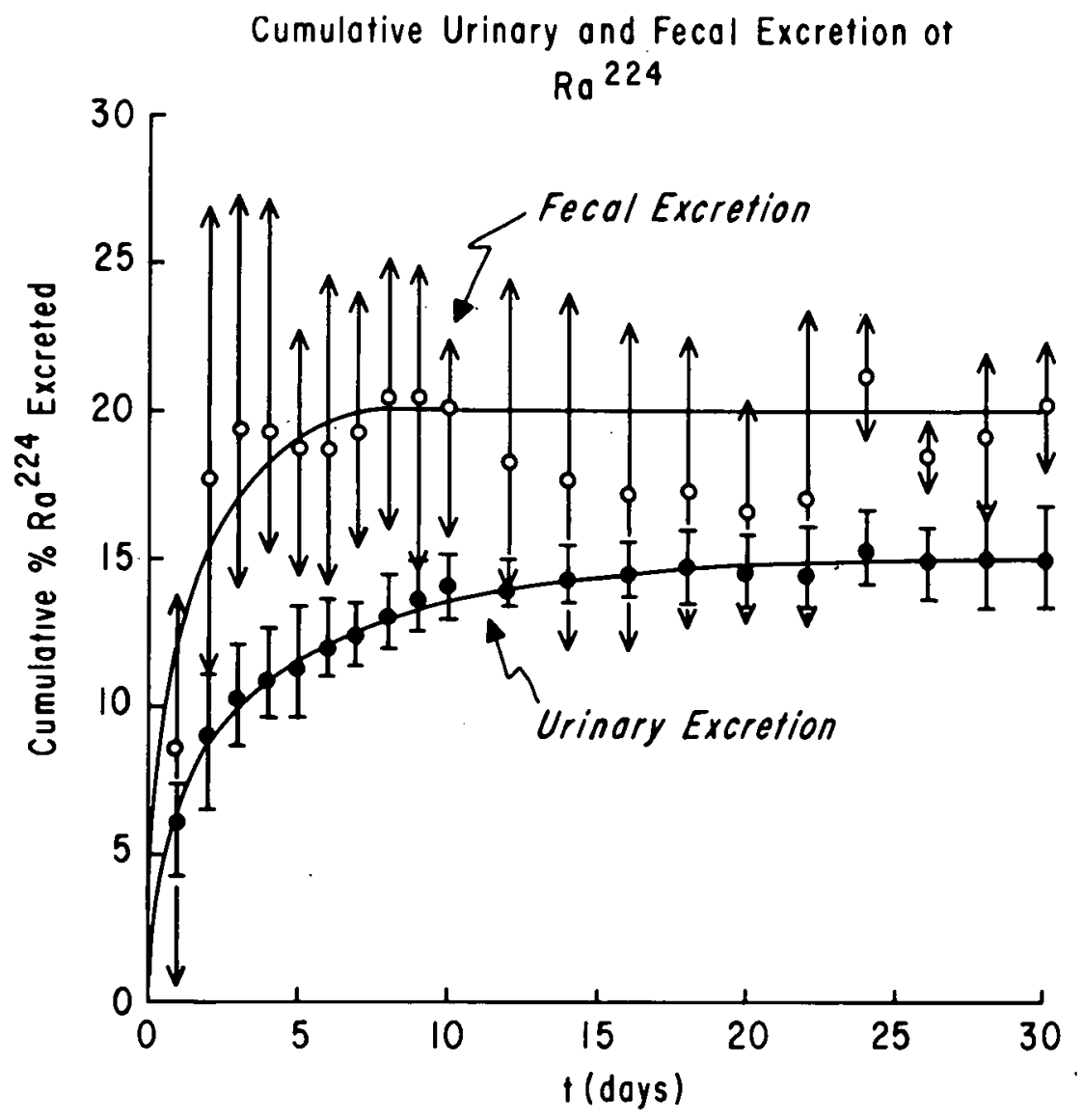

Fig. 3. Cumulative urinary and fecal excretion of $\mathrm{Ra}^{224} \cdot 0-22$ days, mean and range for 4 beagles, 22-30 days, mean and range for 2 beagles. 


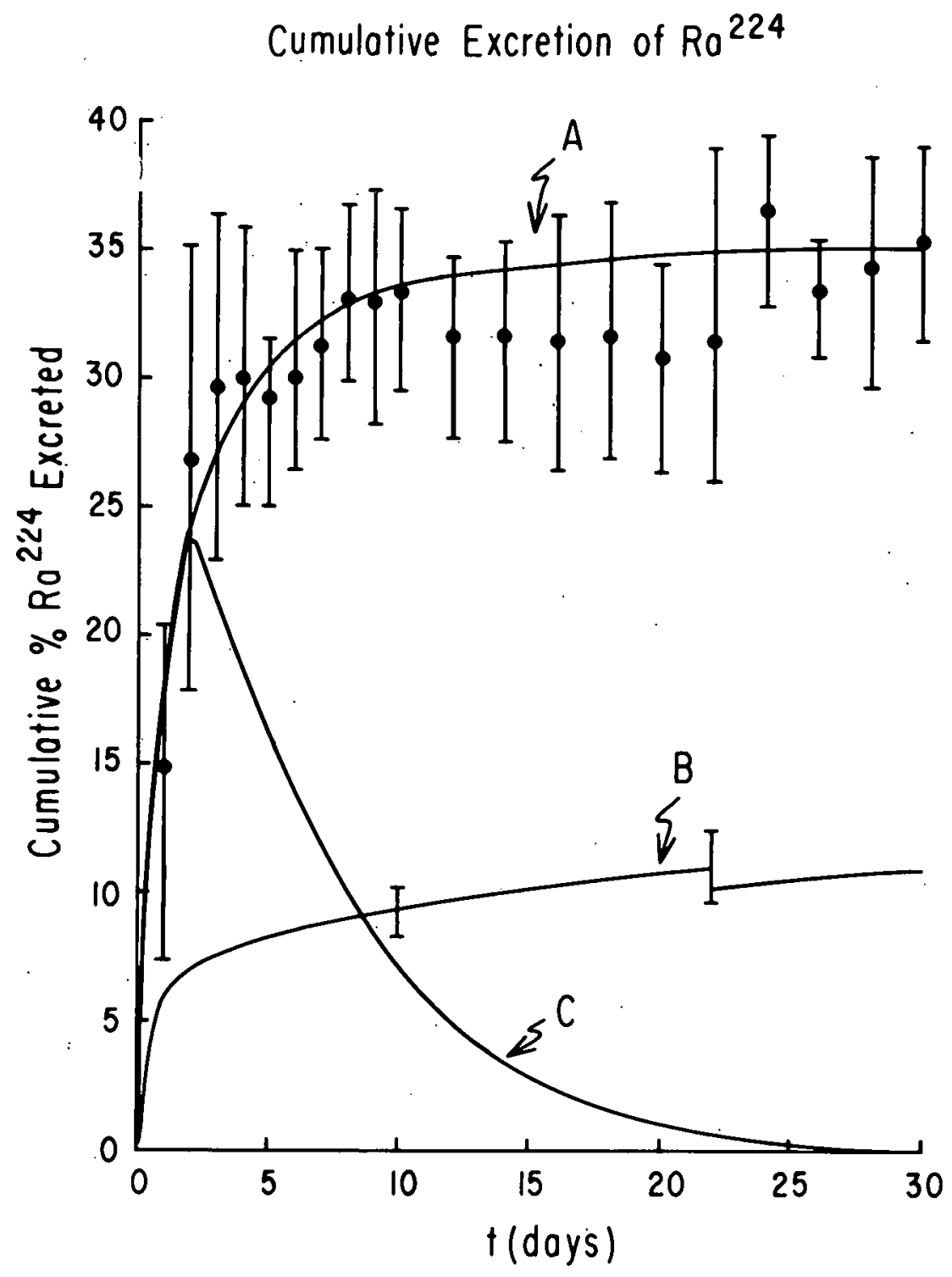

Fig. 4. A. Cumulative excretion of $\mathrm{Ra}^{224} \cdot 0-22$ days, mean and range for 4. beagles, 22-30 days, mean and range for 2 beagles. B. Mean cumulative excretion of $\mathrm{Th}^{228}$ by same dogs. C. Calculat! ed cumulative excretion of injected $\mathrm{Ra}^{224}$. 
of injected $\mathrm{Ra}^{224}$ calculated from $\mathrm{Ra}^{226}$ data and the $\mathrm{Ra}^{224}$ decay factor. Thus, at a month after injection of $\mathrm{Th}^{228}$, about $35 \%$ of the $\mathrm{Ra}^{224}$ is decaying outside the dog but only $11 \%$ of the parent $\mathrm{Th}^{228}$ decays outside, (if the dog is not suffering serious early effects). The fractional retention of $\mathrm{Ra}^{224}$ being formed in vivo at this time is $f=R\left(\mathrm{Ra}^{224}\right)$ / $R\left(\operatorname{Th}^{228}\right)=0.65 / 0.89=0.73$, i.e. 1 out of $4 \mathrm{Ra}^{224}$ atoms formed in the dog is excreted.

Sixty-five measurements of daily urinary and fecal excretion of $\mathrm{Ra}^{224}$ were made during the period 100 to 1260 days after injection. Dogs at dose levels $4\left(0.90 \mu \mathrm{c} \mathrm{Th}^{228} / \mathrm{kg}\right), 3\left(0.30 \mu \mathrm{c} \mathrm{Th}{ }^{228} / \mathrm{kg}\right), 2(0.096$ $\left.\mu \mathrm{c} \operatorname{Th}^{228} / \mathrm{kg}\right), 1.5\left(0.032 \mu \mathrm{c} \mathrm{Th}^{228} / \mathrm{kg}\right)$, and $1\left(0.016 \mu \mathrm{c} \mathrm{Th}^{228} / \mathrm{kg}\right)$ were included. The latest 4- and 3-level measurements were at 832 and 956 days, respectively, which times are close to maximum survival times for these levels. The latest 1-level measurements were at 430 days.

In both urinary and fecal samples the activity of $\mathrm{Ra}^{224}$ was considerably greater than that of $\mathrm{Th}^{228}$. After the first day the gamma count rate varies with time as does the $\mathrm{Ra}^{224}$ activity which is

$$
A_{2}=A_{1}^{o}\left(e^{-\lambda_{1} t}-e^{-\lambda_{2} t}\right)+A_{2}^{0} e^{-\lambda_{2} t}
$$

since $\lambda_{2} /\left(\lambda_{2}-\lambda_{1}\right)=1$. (Subscripts $I$ and 2 denote $\mathrm{Th}^{228}$ and $\mathrm{Ra}^{224}$, respectively) - Rearranging Eq • (10) gives

$$
A_{2}=A_{1}^{0} e^{-\lambda_{1} t}+\left(A_{2}^{O}-A_{1}^{o}\right) e^{-\lambda_{2} t}
$$

Further, the counting is done using a $\operatorname{Th}^{228}$ reference standard which has 
the effect of dividing by $e^{-\lambda} I^{t}$, i.e.

$$
\begin{aligned}
A_{2} e^{\lambda_{1} t} & =A_{1}^{0}+\left(A_{2}^{0}-A_{1}^{O}\right) e^{-\left(\lambda_{2}-\lambda_{1}\right) t} \\
& =A_{1}^{O}+\left(A_{2}^{O}-A_{1}^{O}\right) e^{-\lambda_{2} t}
\end{aligned}
$$

since $\lambda_{2} \gg \lambda_{1}$.

If $C$ is the gamma count rate of the sample relative to that of the $\mathrm{Th}^{228}$ reference standard, then we have

$$
c=a+b e^{-\lambda_{2} t}
$$

From Eqs. (12) and (13),

$$
\mathrm{A}_{1}^{0}=\mathrm{k} \text { a }
$$

and

$$
A_{2}^{0}-A_{1}^{0}=\frac{k b}{\lambda_{3} /\left(\lambda_{3}-\lambda_{2}\right)}
$$

where $k$ is a constant, and, because of the transient equilibrium relation between $\mathrm{Ra}^{224}$ and $\mathrm{Pb}^{212}$, denoted by subscript 3 , b must be reduced as shown. In principle then we can determine $A_{1}^{\circ}$ and $A_{2}^{\circ}$ from the graph of $C$ vs. $t$. In practice, however, the excretion of $\operatorname{Th}^{228}$ was too low to be determined precisely in this way, so that, subsequent to gamma counting, the samples were ashed and $\mathrm{Th}^{228}$ was determined, ultimately, by $\alpha_{-}$ counting (2). The component a proved to be a measure of contamination . which included $\mathrm{Th}^{228}$ and any. traces of gamma emitters present in the 
sample intrinsically or from cages, containers, etc. Thus, determination of a gave no direct information, but it was necessary to have a to obtain $\underline{\mathrm{b}}$ accurately.

Hence, from gamma counting we obtain $A_{2}^{O}-A_{1}^{O}$, the amount by which $\mathrm{Ra}^{224}$ activity exceeds $\mathrm{Th}^{228}$ activity. The procedure in working up these data has been to fit equations to $A_{2}^{O}-A_{1}^{O}$ to obtain, for example,

$$
U^{\prime}\left(\operatorname{Ra}^{224}\right)=U\left(\operatorname{Ra}^{224}\right)-U\left(\operatorname{Th}^{228}\right)=a e^{-b t}
$$

Then $U\left(T^{228}\right)$ from Ref. (2) is added to Eq. (14) to obtain U(Ra $\left.{ }^{224}\right)$.

The results are given in the three graphs of Fig. 5. The 4-level dogs exhibited marked skeletal and kidney damage (I) and this was reflected in decreased excretion of $\mathrm{Ra}^{224}$. A similar effect on urinary excretion of $\mathrm{Th}^{228}$ by 4 -level dogs has been reported (2). Equations for the 9. curves shown in Fig. 5 are as follows:

$$
\begin{array}{ll}
U^{\prime}=0.656 \mathrm{e}^{-0.00092 t} & \text { (all levels) } \\
U^{\prime}=0.742 \mathrm{e}^{-0.00091 t} & \text { (all but 4) } \\
\mathrm{U}^{\prime}=0.640 \mathrm{e}^{-0.00191 \mathrm{t}} & \text { (4 only) } \\
F^{\prime}=1.14 \mathrm{e}^{-0.00053 t} & \text { (all levels) } \\
F^{\prime}=1.15 \mathrm{e}^{-0.00048 t} & \text { (all but 4) } \\
\mathrm{F}^{\prime}=1.36 \mathrm{e}^{-0.00124 t} & \text { (4 only) } \\
X^{\prime}=1.85 \mathrm{e}^{-0.00075 t} & \text { (all levels) } \\
X^{\prime}=1.94 \mathrm{e}^{-0.00072 t} & \text { (all but 4) } \\
X^{\prime}=2.02 \mathrm{e}^{-0.00143 t} & \text { (4 only) }
\end{array}
$$




\section{A. Urinary Excretion}
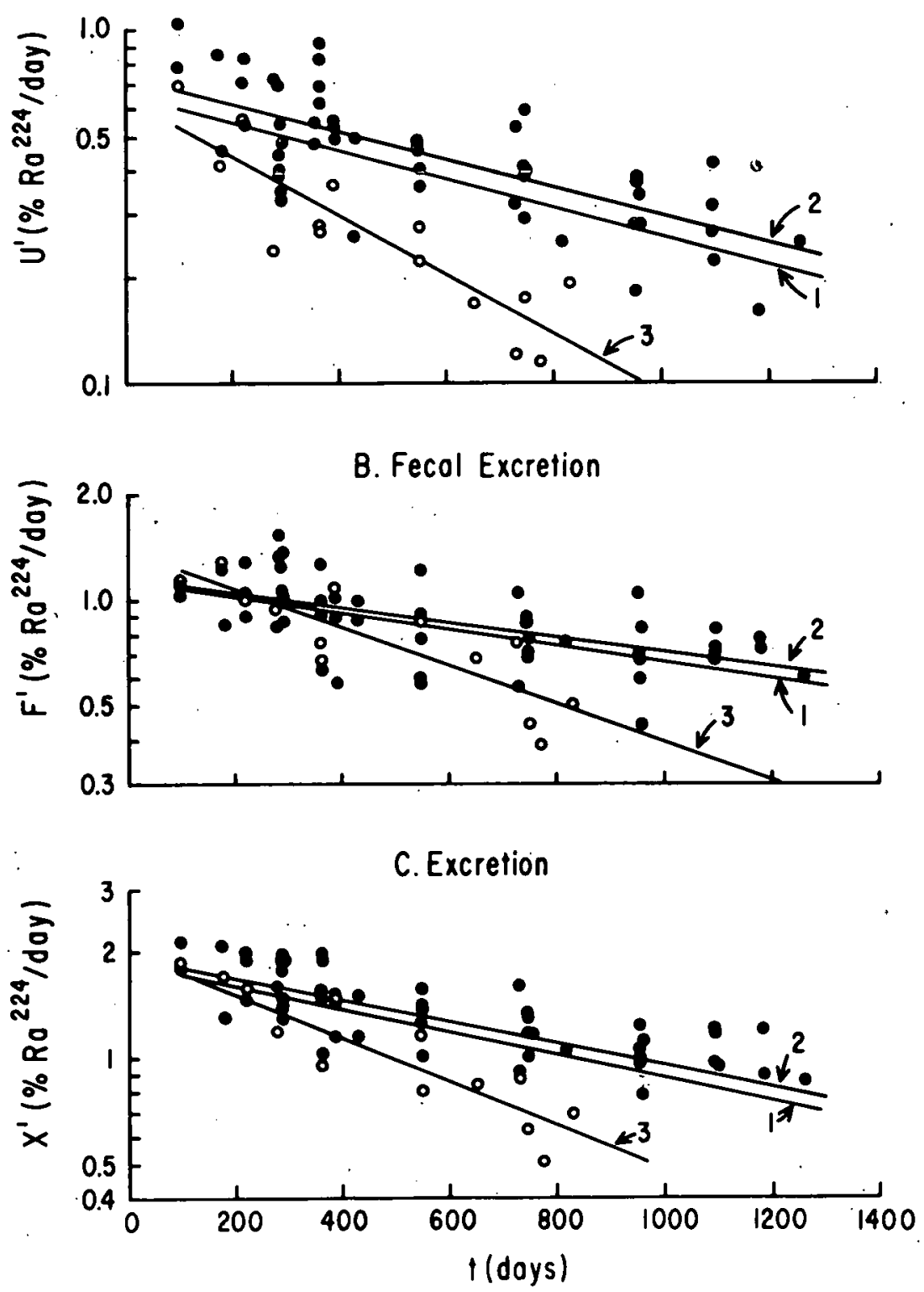

Fig. 5. (\% $\mathrm{Ra}^{224}-\% \mathrm{Th}^{228}$ ) excreted per day. A. Uninary, B. Fecal, and C. Urinary plus fecal excretion. On each graph curve 1 was calculated using all points, curve 2 using level-1, level1.5, level-2, and level-3 points and curve 3 using level-4 points only. 
Units are \% $\mathrm{Ra}^{224}$ /day and days. The $90 \%$ symmetrical confidence limits for each $\log a$ and each $b$ appear in Table $I$. The limits for log a may at first be misleading. For example, consider Eq. (20), F (all), for which $\log a=0.0564 \pm 0.1139$. The limits on $a=1.14$ are the antilogs of $0.0564-0.1139$ and $0.0564+0.1139$ which are 0.88 and 1.48 , respectively.

The coefficients of $t$ in the exponentials (the $b^{\text {'s }}$ ) of Eqs. (17-25) were compared using the t-test. In all three cases (U, F, and $X$ ) the values for the "all" and "all but 4" groups did not differ, but those for the "all" and "4 only" groups did differ, $p<0.01$.

During this period, 100-1260 days, excretion of $\mathrm{Th}^{228}$ is much lower, and the urinary rate decreases twice as fast while the total rate decreases about $1-1 / 2$ times as fast (2) . The equations for the "all" group are

$$
\mathrm{U}\left(\mathrm{Th}^{228}\right)=0.019 \mathrm{e}^{-0.0018 t}
$$

and

$$
X\left(\operatorname{Th}^{228}\right)=0.021 \mathrm{e}^{-0.0011 t}
$$

The $\operatorname{Th}^{228}$ data were worked up in the dark ages of log tables and desk calculators, and for this reason a separate equation for $\mathrm{F}\left(\mathrm{Th}^{228}\right.$ ) was not obtained.

Combining Eqs: (17) and (26) in the manner of Eq. (16) we obtain for the daily urinary excretion for all levels 


$$
\begin{aligned}
& \vdots \\
& U\left(\operatorname{Ra}^{224}\right)=0.656 \mathrm{e}^{-0.00092 t}+0.019 \mathrm{e}^{-0.0018 t} \\
& \simeq 0.675 \mathrm{e}^{-0.00092 t}
\end{aligned}
$$

for the period of interest. Similarly the total daily rate from Eqs.

(23) and (27) is

$$
\begin{gathered}
X\left(\operatorname{Ra}^{22 t}\right)=1.85 \mathrm{e}^{-0.00075 t}+0.021 \mathrm{e}^{-0.0011 t} \\
\simeq 1.87 \mathrm{e}^{-0.00075 t}
\end{gathered}
$$

The important question to be answered from the excretion studies is what fraction of the $\mathrm{Ra}^{224}$ formed in the dog is decaying outside the dog from 100 to 1260 days. This result is easily computed from Eq. (29) for $X$ since the rate of physical decay of $\mathrm{Ra}^{224}\left(\lambda=0.191 \mathrm{~d}^{-1}\right)$ is much greater than the rates at which the daily excretion of $\mathrm{Ra}^{224}$ and $\mathrm{Th}^{228}$. change. These are

$$
\operatorname{dln} X\left(\operatorname{Ra}^{224}\right) / d t=-0.00075
$$

and

$$
\mathrm{d} \ln X\left(\operatorname{Th}^{228}\right) / \mathrm{dt}=-0.0011
$$

Therefore, these changes can be neglected in summing to obtain cumulative excretion. On a given day $\mathrm{T}$ in the period 100 to 1260 days, the excretion for that day is $X(T)=1.87 \mathrm{e}^{-0.00075 T}$. On the $\mathrm{T}$-1st day $\mathrm{X}(\mathrm{T}-1)$ was excreted and by $T$ it has been reduced by decay by $e^{-\lambda}$ so that $X(T-1)$ $\mathrm{e}^{-\lambda}=1.87 \mathrm{e}^{-0.00075(\mathrm{~T}-1)} \mathrm{e}^{-0.191}=1.87 \mathrm{e}^{-0.0075 \mathrm{~T}} \mathrm{e}^{-0.191+0.00075}$ 
$\cong X(T) e^{-\lambda}$ (since $0.00075 \therefore 0.191$ ) gives the $\operatorname{Ra}^{224}$ outside the dog as a result of the T-1st day's excretion. Similarly the T-2nd days excretion contributes $X(T) e^{-2 \lambda}$. The total $\mathrm{Ra}^{224}$ which is formed in the dog but excreted to decay outside the dog, $\Sigma \dot{x}$, is thus given by

$$
\begin{aligned}
\because X(T) & =X(T)+X(T) e^{-\lambda}+X(T) e^{-2 \lambda}+\cdots \\
& =X(T)\left(1+e^{-\lambda}+e^{-2 \lambda}+\cdots\right. \\
& =X(T) /\left(1-e^{-\lambda}\right)=5.75 X(T)
\end{aligned}
$$

The biological retention of $\mathrm{Ra}^{224}$ is given by

$$
\begin{aligned}
R_{e}\left(\operatorname{Ra}^{224}\right) & =R_{e}\left(\mathrm{Th}^{228}\right)-5.75 \times\left(\mathrm{Ra}^{224}\right) \\
& =19.1 \mathrm{e}^{-0.0011 t}+67.9-10.8 \mathrm{e}^{-0.00075 t}
\end{aligned}
$$

and the actual retention is

$$
\begin{gathered}
R_{e}^{\prime}\left(\operatorname{Ra}^{224}\right)=R_{e}\left(R a^{224}\right) e^{-\lambda} \operatorname{Th}^{228 t}= \\
19.1 e^{-0.0021 t}+67.9 e^{-0.0010 t}-10.8 e^{-0.00175 t}
\end{gathered}
$$

where $\mathrm{R}_{\mathrm{e}}\left(\mathrm{Th}^{228}\right)$ was obtained from excretion data (2). If we use the probably better estimate of $\mathrm{Th}^{228}$ retention derived from analyses of selected bones and soft tissues plus 9 carcasses (2), we have

$$
R\left(\operatorname{Ra}^{224}\right)=18 e^{-0.0010 t}+69 e^{-0.00014 t}-10.8 e^{-0.00075 t}
$$

and

$$
R^{\prime}\left(R a^{224}\right)=18 e^{-0.0020 t}+69 e^{-0.00114 t}-10.8 e^{-0.00175 t}
$$


For times greater than about a year Eq. (35) appears to be better than Eq. (33) . Earlier they are about equivalent.

From Eq. (35) we calculate the fractional retention of $\mathrm{Ra}^{224}$ formed in vivo to be

$$
\begin{aligned}
f & =\frac{R\left(\operatorname{Ra}^{224}\right)}{R\left(\operatorname{Th}^{228}\right)}=1-\frac{10.8 \mathrm{e}^{-0.00075 t}}{18 \mathrm{e}^{-0.0010 t}+.69 \mathrm{e}^{-0.00014 t}} \\
& =1-\frac{1}{1.67 \mathrm{e}^{-0.00025 t}+6.39 \mathrm{e}^{+0.00061 t}}
\end{aligned}
$$

Thus, $f$ approaches unity as $t$ increases. At 100, 500, and 1000 days Eq. (37) gives $f=0.88,0.90$, and 0.92 , respectively. In the early studies $\mathrm{f} 0.73$ at 30 days. This observed value is lower than would be calculated from Eq. (37) derived from data obtained at later times, which is illustrative of the difference in the nature of the $\mathrm{Th}^{228}$ deposition at short and long times.

If we use the model of Van Dilla et al., which assumes that $\mathrm{Ra}^{224}$ formed in vivo will, on reaching the blood, be excreted and redistributed as though it had been. injected intravenously, (3), we can calculate from $f$ the fraction of $\mathrm{Ra}^{224}$ which undergoes translocation. First we must calculate $R\left(\mathrm{Ra}^{224}\right) / \mathrm{R}\left(\mathrm{Th}^{228}\right)=\mathrm{f}$ f for the case in which every atom of $\mathrm{Ra}^{224}$ formed in vivo goes to the blood. This model also assumes .that a $\mathrm{Ra}^{224}$ atom goes to blood shortly, i.e.<< 3.64 days after its formation.

Let $A_{1}^{0}=$ activity of $\operatorname{Th}^{228}$ injected at $t=0$. At $t>0$ the activity of $\mathrm{Th}^{228}$ in the dog is 


$$
A_{1}(\operatorname{dog})=A_{1}^{0} e^{-\lambda_{1} t} R_{1}(t)
$$

where $R_{l}(t)=R\left(T^{228}\right)$ above: The number of atoms of $R a^{224}$ formed in vivo during the interval $x$ to $x+d x$ is

$$
A_{1}(\operatorname{dog}) d x=A_{1}^{O} e^{-\lambda} I^{x} R_{1}(x) d x
$$

At some time later these will be reduced by decay and excretion to

$$
A_{1}^{o} e^{-\lambda l^{x}} R_{1}(x) e^{-\lambda_{2}(t-x)} R_{2}(t-x) d x
$$

Multiplying by $\lambda_{2}$ and integrating from $x=0$ to $x=t$ gives the activity of $\mathrm{Ra}^{224}$ in the dog at $\mathrm{t}$ :

$$
A_{2}(\operatorname{dog})=\lambda_{2} \int_{0}^{t} A_{1}^{0} e^{-\lambda_{1} x} R_{1}(x) e^{-\lambda_{2}(t-x)} R_{2}(t-x) d x
$$

as noted above both the rate of decay and the rate of decrease of $R\left(\operatorname{Th}^{228}\right)$ after a few weeks are small compared with the rate of decay of $\mathrm{Ra}^{224}$ so that we introduce no significant error by taking $A_{1}(d \circ g)$ outside the integral sign. For $R_{2}(t)$ we use the equation for $\mathrm{Ra}^{226}$ retention in beagles (6):

$$
R=0.79 t^{-0.20}
$$

These lead to the relation 


$$
\begin{aligned}
& \left(\frac{A_{2}}{A_{1}}\right)_{d o g}=\frac{R\left(R^{224}\right)}{R\left(T^{228}\right)}=f^{*}= \\
& \lambda_{2} 0.79 \int_{0}^{t} e^{-\lambda_{2}(t-x)}(t-x)^{-0.20} d x \\
& \quad=\lambda_{2} 0.79 \int_{0}^{t} e^{-\lambda_{2} z} z^{-0.20} d z .
\end{aligned}
$$

Where $z=t-x$. Again because of the relatively rapid rate of decay we can let $t \rightarrow \infty$ so that

$$
\begin{aligned}
& f *=\frac{\lambda_{2} 0.79}{\lambda_{2}(-0.20+1)} \Gamma(-0.20+1)= \\
& 0.79(0.191)^{0.20} \Gamma(0.80)= \\
& \frac{0.79(0.191)^{0.20}}{0.80} \Gamma(1.81)= \\
& \frac{0.79 \times 0.931}{0.80 \times 1.39}=0.66
\end{aligned}
$$

Thus, if all $\mathrm{Ra}^{224}$ atoms formed in the dog moved to the blood about $1 / 3$ of them would be excreted since

$$
1-f *=0.34
$$

The observed values of l-f are less than 0.34 which means that within 
the limits of the model used, only part of the $\mathrm{Ra}^{224}$ translocates. The fraction which translocates $F_{t}\left(\operatorname{Ra}^{224}\right)$ is given by

$$
\mathrm{F}_{t}\left(\mathrm{Ra}^{224}\right)=\frac{1-\mathrm{f}}{1-\mathrm{f}^{*}}
$$

$F_{t}\left(R^{224 ;}\right.$ calculated from excretion data, i.e. Eqs. (37) and (44), decreases slowly with time as shown in Fig. 6. Using Eq. (25) the same calculation has been made for 4-level dogs only. Since $F_{t}\left(\mathrm{Ra}^{224}\right)$ for 4-level dogs decreases more rapidly than, that calculated for all the dogs, radiation damage (probably skeletal and renal) is a factor in the decrease of translocation of $\mathrm{Ra}^{224}$.

Mays et al. (4) obtained relations for percent thoron exhalation (relative to in vivo produced thoron) for dose levels 4, 3, and 2 and lower. Choosing their 3 -level equation as representative of all the levels and converting to our units we obtain

$$
\begin{gathered}
\% \operatorname{Tn}^{220} \text { exhaled }=8 t^{-0.17}\left(0.93 t^{-0.023}-0.47 t^{-0.31}\right) \\
=7.4 t^{-0.19}-3.8 t^{-0.31} \simeq 2.5 \mathrm{e}^{-0.0003 t}
\end{gathered}
$$

The approximation to the exponential was obtained graphically and is quite good for $t=200$ to 1200 days. Thus, for thoron retention we have

$$
\begin{aligned}
R\left(\operatorname{Tn}^{220}\right) & =R\left(\operatorname{Ra}^{224}\right)-2.5 \mathrm{e}^{-0.0003 t} \\
& \cong 0.98 \mathrm{R}\left(\mathrm{Ra}^{224}\right)
\end{aligned}
$$




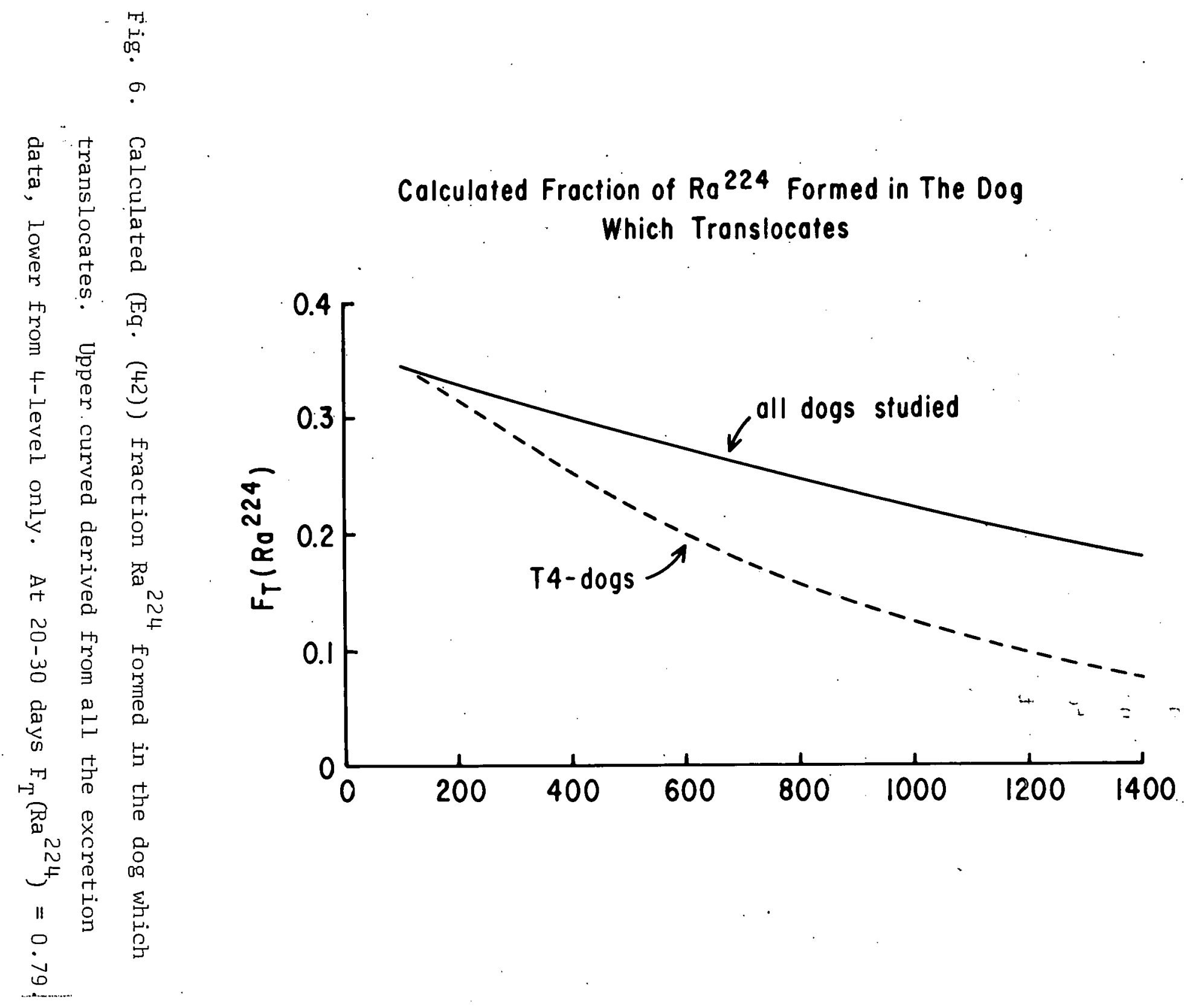


An extensive study of the excretion of $\mathrm{Pb}^{212}$ comparable with the $\mathrm{Ra}^{224}$ studies would have required such enormous amounts of technical time that it could not be justified. Because of the 10.6 hour half period it would be necessary to collect excreta every few hours around the clock in. order to obtain an accurate value for $\mathrm{Pb}^{212}$ in the sample at the time of excretion. Then properly accumulating results from these samples would give the $\% \mathrm{~Pb}^{212}$ formed in the dog which decayed outside the dog.

A further complication in determining urinary excretion of $\mathrm{Pb}^{212}$ is that in a freshly voided sample the activity of $\mathrm{Bi}^{212}$ is high relative to both $\mathrm{Pb}^{212}$ and $\mathrm{Ra}^{224}$. Thus the counting schedule must be planned to obtain sufficient measurements after several $\mathrm{Bi}^{212}$ half-periods but within the first' 1 to $2 \mathrm{~Pb}^{212}$ half-periods.

We do have findings from excretion studies that indicate most of the $\mathrm{Pb}^{212}$ formed in the dog decays there. When beagles were given intravenous injections of $\mathrm{Pb}^{212}$, the biological excretion at 24 hours was only $4 \%$, and urinary excretion of 5 times fecal excretion (7) .

In addition, two short.series of freshly voided urines from a 3level $\mathrm{Th}^{228}$ dog were counted to determine the relative activities of $\mathrm{Ra}^{224}, \mathrm{~Pb}^{212}$, and $\mathrm{Bi}^{212}$.

The count rate data in these two studies were resolved using a Gauss-Siedel iterative procedure of least squares analysis on an IBM 7090 computer. This method has been described by Richmond et al. (8) .

In the first series dog T8T3, houséd in a metabolism cage, was given a bowl of milk at the beginning of the work day, and then the cage was checked at 30-minute intervals for urine. When the first urine was 
found it was sealed in an ampoule and serial counting was begun 15 to 20 minutes after collection. A sample was obtained on each of three successive mornings. The activities of $\mathrm{Ra}^{224}, \mathrm{~Pb}^{212}$, and $\mathrm{Bi}^{212}$ were all considerably greater than that of $\mathrm{Th}^{228}$ and, for reasons given above, the results are expressed as

$$
\begin{gathered}
\mathrm{U}^{\prime}\left(\mathrm{Ra}^{224}, \mathrm{~Pb}^{212} \text {, or } \mathrm{Bi}^{212}\right)= \\
\mathrm{U}\left(\mathrm{Ra}^{224}, \mathrm{~Pb}^{212} \text {, or } \mathrm{Bi}^{212}\right)-\mathrm{U}\left(\mathrm{Th}^{228}\right)
\end{gathered}
$$

Further, it was assumed that the urine was excreted at the mid-point of the 30 minutes between cage checks and the activity of $\mathrm{Bi}^{212}$ was corrected for 15 minutes decay. The results are given in Table III and clearly show that some $\mathrm{Pb}^{212}$ and $\mathrm{Bi}^{212}$ formed in the dog decays outside the dog.

\section{TABLE III}

Activities of $\mathrm{Pb}^{212}$ and $\mathrm{Bi}^{212}$ Relative
to $\mathrm{Ra}^{224}$ in Freshly Voided Urine

\begin{tabular}{ccc}
\hline \hline $\begin{array}{l}\text { Days After } \\
\text { Injection }\end{array}$ & $\mathrm{U}^{\prime}\left(\mathrm{Pb}^{212} / \mathrm{U}^{\prime}\left(\mathrm{Ra}^{224}\right)\right.$ & $\mathrm{U}^{\prime}\left(\mathrm{Bi}^{212}\right) / \mathrm{U}^{\prime}\left(\mathrm{Ra}^{224}\right)$ \\
\hline 671 & 1.72 & 18.0 \\
672 & 1.04 & 9.2 \\
673 & 1.10 & 11.2 \\
\hline
\end{tabular}

In the second series, to obtain a more quantitative relation between $\mathrm{Pb}^{212}, \mathrm{Bi}^{212}$, and $\mathrm{Ra}^{224}$ urinary excretion, samples were collected over a 24 hour interval. Dog T8T3 was given water and milk at 0830 hours 777 
days after injection and urine was collected at 2 hour intervals for 12 hours and at the end of 24 hours. The activities of the 3 nuclides in each sample at time of collection are given in Table IV. Also, given are the cumulative activities for each time. At 678 days the measured daily urinary excretion of $\mathrm{Ra}^{22 \cdot 4}$ by this dog was

$$
\mathrm{U}^{\prime}\left(\mathrm{Ra}^{224}\right)=0.252
$$

TABLE IV

Cumulative Urinary Excretion of $\mathrm{Ra}^{224}, \mathrm{~Pb}^{212}$, and $\mathrm{Bi}^{212}$. From 0830 Hours 777 Days After Injection to 0830778 Days

\begin{tabular}{|c|c|c|c|c|c|c|}
\hline \multirow{2}{*}{$\stackrel{t}{\text { Hours }}$} & \multicolumn{2}{|c|}{$\mathrm{Ra}^{224}$} & \multicolumn{2}{|c|}{$\mathrm{Pb}^{212}$} & \multicolumn{2}{|c|}{$B i^{212}$} \\
\hline & Sample & Cumulative & Sample & Cumulative & Sample & Cumulative \\
\hline 0 & 0 & 0 & 0 & 0 & 0 & 0 \\
\hline 2 & 0.011 & 0.011 & 0.022 & 0.022 & 0.27 & 0.27 \\
\hline 4 & 0.020 & 0.031 & 0.030 & 0.049 & 0.30 & $0.37^{\circ}$ \\
\hline 6 & 0.036 & .0 .0666 & 0.032 & 0.075 & 0.36 & 0.45 \\
\hline 8 & 0.031 & 0.096 & 0.059 & 0.124 & .0 .42 & 0.54 \\
\hline 10 & 0.032 & 0.126 & 0.063 & 0.172 & 0.39 & 0.53 \\
\hline 12 & 0.038 & 0.162 & $0.087^{\circ}$ & 0.242 & 0.78 & 0.91 \\
\hline 24 & 0.081 & 0.228 & 0.117 & 0.227 & -- & -- \\
\hline
\end{tabular}

The cumulative value from the seven samples spanning a day is about the same, 0.228. Thus apparently there was no marked effect of the extra liquid given the dog at the beginning of the period.

The: cumulative values shown for $\mathrm{Pb}^{212}$ are slightly high because some $\mathrm{Pb}^{212}$ grew in from $\mathrm{Ra}^{224}$ after the sample was voided but before 
collection time. If we assume that the urine was voided at the midpoints of the collection intervals, and correct accordingly, the 24 hour cumulative value is 0.201 , which does not differ much from 0.227 . For estimating \% $\mathrm{Pb}^{212}$ formed in the dog which decays outside, this correction is small enough to be ignored. Summing as in Eq. (32) gives

$$
\Sigma \mathrm{U}^{\prime}\left(\mathrm{Pb}^{212}\right)=\frac{0.227}{1-\mathrm{e}^{-\lambda} \mathrm{Pb}^{212}} \cong 0.4
$$

Fecal excretion was not measured, but from the intravenous $\mathrm{Pb}^{212}$ studies it is expected to be less than the urinary excretion. However, the urinary to fecal ratio is probably. lower in these dogs where $\mathrm{Pb}^{212}$. is being formed continously in the blood. These limited findings show that a small amount of $\mathrm{Pb}^{212}$ is excreted, and we estimate $\mathrm{Pb}^{212}$ retention to be

$$
\mathrm{R}\left(\mathrm{Pb}^{212}\right) \simeq 0.99 \mathrm{R}\left(\mathrm{Tn}^{220}\right)=0.97 \mathrm{R}\left(\mathrm{Ra}^{224}\right)
$$

The cumulative $\mathrm{Bi}^{212}$ excretion from 6 to 12 hours is the steady state value for \% $\mathrm{Bi}^{212}$ formed $\underset{i}{\mathrm{n}}$ the dog but decaying outside, since 6 hours is 6 half-periods for $\mathrm{Bi}^{212}$. Again the correction for $\mathrm{Bi}{ }^{212}$ growth from $\mathrm{Pb}^{21.2}$ after voiding has not been made. Since the activity of $\mathrm{Bi}^{212}$ is about 10 times that of $\mathrm{Pb}^{212}$, the correction to the mid-point of the 2 hour interval would be about 5\%, which is negligible. Fecal excretion is assumed negligible because of the short half-period. Thus,

$$
\Sigma X^{\prime}\left(\mathrm{Bi}^{212}\right) \sim 0.45 \text { to } 0.91
$$


and we estimate retention as

$$
R\left(B i^{212}\right) \sim 0.99 R\left(\mathrm{~Pb}^{212}\right)=0.96 \mathrm{R}\left(\mathrm{Ra}^{224}\right)
$$

In Table $V$ appear retention estimates (both biological, $R$, and actual, $\mathrm{R}^{\prime}$ ), for the $\mathrm{Th}^{228}$ series at 100 and 1000 days. In comparison, whole body $\gamma$-counting data gave an average of

$$
R\left(B i^{212}\right)=0.52
$$

from 10 to 1800 days (1).

TABLE V

Estimated Retention of $\mathrm{Th}^{228}$ Series

\begin{tabular}{|c|c|c|c|c|}
\hline \multirow[b]{2}{*}{ Nuclide } & \multicolumn{2}{|c|}{$t=100$} & \multicolumn{2}{|c|}{$t=1000$} \\
\hline & $\underline{R}$ & $\dot{R}^{\prime}$ & $\mathrm{R}$ & $\mathrm{R}^{\prime}$ \\
\hline $\mathrm{Th}^{228}$ & 85.0 & 77.0 & 66.6 & 24.4 \\
\hline $\mathrm{Ra}^{224}$ & 75.0 & 68.0 & 61.5 & 22.6 \\
\hline $\operatorname{Tn}^{220}$ & 73.5 & 66.5 & 60.3 & 22.1 \\
\hline $\mathrm{Pb}^{212}$ & .72 .6 & 65.6 & 59.6 & 21.8 \\
\hline$B i^{212}$ & 72.0 & 65.2 & 59.0 & 21.6 \\
\hline
\end{tabular}

at 100 and 1000 Days

Liver. The activities of $\mathrm{Ra}^{224}$ and $\mathrm{Pb}^{212}$, were determined in 14 of the 22 liver samples in which $\operatorname{Th}^{228}$ was measured (2). The results are given both in Fig. 7 and Table VI. Included in the latter are some of: the important clinical observations made at time of euthanasia. The clinical status of the dogs is perhaps the most serious limitation of the data. 

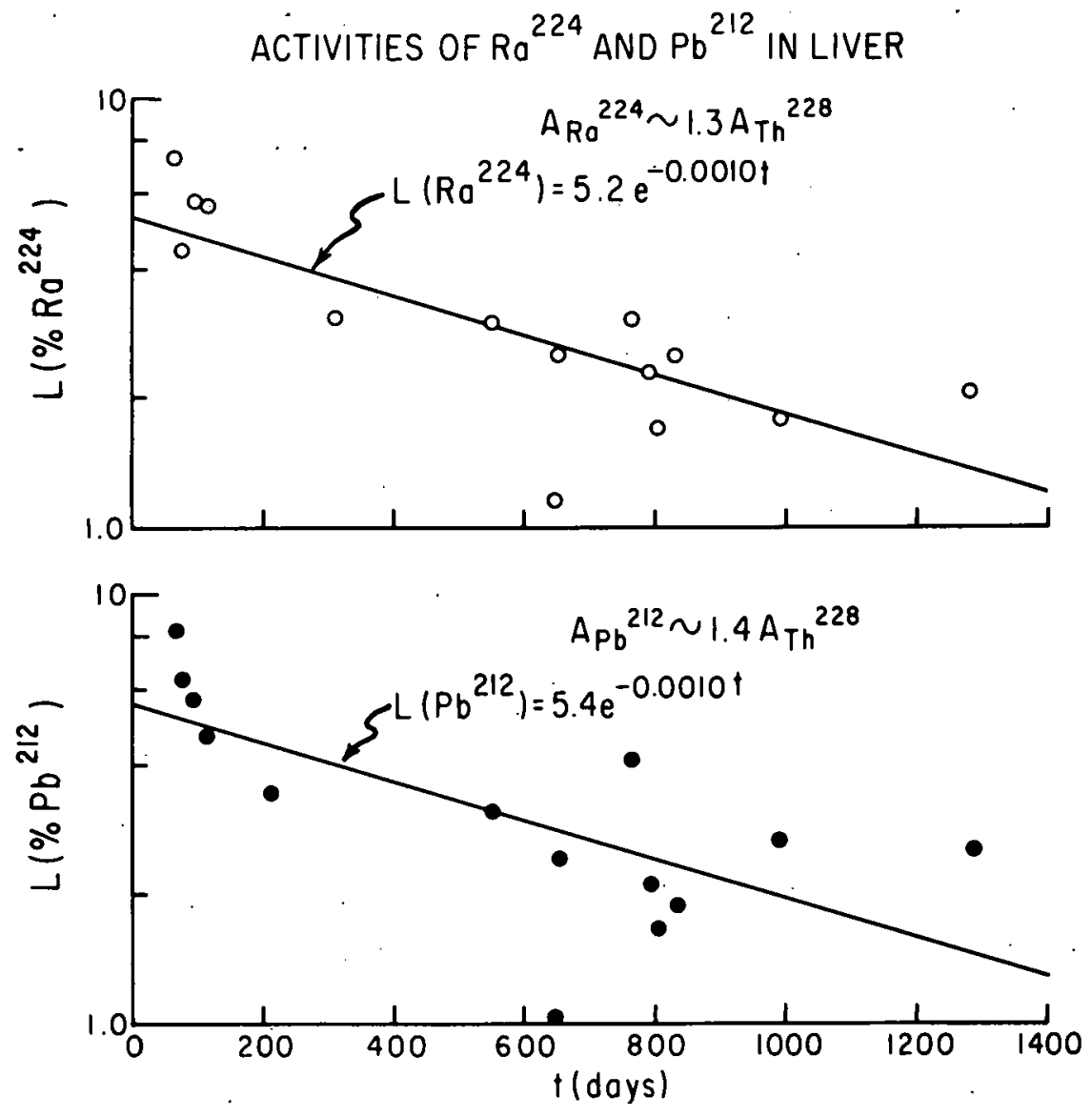

Fig. 7. Activities of $\mathrm{Ra}^{224}$ and $\mathrm{Pb}^{212}$ in liver. 
TABLE VI

Activities of $\mathrm{Th}^{228}, \mathrm{Ra}^{224}$, and $\mathrm{Pb}^{212}$.

in Liver

\begin{tabular}{lccccl}
\hline \hline & $\begin{array}{c}\text { Days After } \\
\text { Injection }\end{array}$ & $\% \mathrm{Th}^{228}$ & $\% \mathrm{Ra}^{224}$ & $\% \mathrm{~Pb}^{212}$ & Comments (1) \\
\hline T5T5 & 6.5 & 6.4 & 7.2 & 8.2 & thrombocytopenia \\
M4T2 & 78 & 5.3 & 4.4 & 6.4 & trauma, shock \\
M2T5 & 97 & 5.7 & 5.7 & 5.7 & pancytopenia \\
T4T5 & 113 & 4.3 & 5.6 & 4.7 & thrombocytopenia \\
M1T5 & 212 & 2.6 & 3.1 & 3.4 & nephritis \\
F3T3 & 547 & 2.2 & 3.0 & 3.1 & osteosarcoma \\
M1T4 & 645 & 0.68 & 1.2 & 1.0 & crippling fractures \\
T6T4 & 651 & 3.6 & 2.5 & 2.4 & crippIing fractures \\
F3T4 & 763 & 1.7 & 3.1 & 4.1 & ulcerative gingivitis \\
M4T4 & 793 & 0.96 & 2.3 & 2.1 & ulcerative gingivitis \\
M4T3 & 801 & 1.4 & 1.7 & 1.8 & osteosarcoma \\
M2T4 & 833 & 1.1 & 2.5 & 1.9 & ostepsarcoma \\
M1T3 & 988 & 1.5 & 1.8 & 2.6 & osteosarcoma \\
M1T2 & 1282 & 3.9 & 2.0 & 2.5 & osteosarcoma \\
& & & & & \\
\hline Fractional counting errors high. & & & \\
\hline \hline
\end{tabular}

The three nuclides, $\mathrm{Th}^{228}, \mathrm{Ra}^{224}$, and $\mathrm{Pb}^{212}$ were in (or close to) equilibrium in only one case. In 10 samples $\mathrm{Ra}^{224}$ activity exceeded that of $\mathrm{Th}^{228}$. It was lower for $\mathrm{M} 4 \mathrm{~T} 2, \mathrm{~T} 6 \mathrm{~T} 4$, and $\mathrm{MlT} 2$. M4T2 died in shock the day after severe injury in a dog fight. Thus, in a few cases some of the $\mathrm{Ra}^{224}$ produced in the liver is moved out, while in most of the dogs some $\mathrm{Ra}^{224}$ formed elsewhere in the animal is moved into the liver. The equation for $\mathrm{Ra}^{224}$ in liver is

$$
L\left(\operatorname{Ra}^{224}\right)=5.2 e^{-0.0010 t}
$$


which shows the same time dependence as that for $\operatorname{Th}^{228}$ (2),

$$
L\left(\operatorname{Th}^{228}\right)=4.0 e^{-0.00094 t}
$$

$\mathrm{Pb}^{212}$ activity exceeded that of $\mathrm{Th}^{228}$ in 11 samples and $\mathrm{Ra}^{224}$ in 8 . The equation for $\mathrm{Pb}^{212}$ in liver is

$$
L\left(\mathrm{~Pb}^{212}\right)=5.4 \mathrm{e}^{-0.0010 t}
$$

Eqs. (4) and (6) for $\mathrm{Ra}^{224}$ in plasma and $\mathrm{Pb}^{212}$ in blood also show the same time dependence. This simplifies answering the question of the effect of residual blood on the determination of $\mathrm{Ra}^{224}$ and $\mathrm{Pb}^{212}$ in liver. The 14 livers weighed $335 \pm 94 \mathrm{~g}$, so that the concentration of $\mathrm{Ra}^{224}$ in plasma relative to liver is only

$$
\frac{5.6 \times 10^{-4}}{5.2 / 335}=0.036
$$

and the effect is negligible. However, the concentration of $\mathrm{Pb}^{212}$ in biood cells relative to liver is

$$
\frac{0.011}{5.4 / 335}=0.68
$$

Thus, some of the $\mathrm{Pb}^{212}$ measured may be associated with blood cells, and it is likely that some of the variation observed in $\mathrm{Pb}^{212}$ results from differences in degree of exsanguination of the dog.

Spleen. The activities of $\mathrm{Ra}^{224}$ and $\mathrm{Pb}^{212}$ were determined in 11 of the spleens in which $\operatorname{Th}^{228}$ was measured. The results appear both in 
Fig. 8 and Table VII. $\mathrm{Ra}^{224}$ activity was less than $\mathrm{Th}^{228}$ activity in 3 spleens, about the same in 3, and higher in 5. The equation is essen-: tially the same as that for $\mathrm{Th}^{228}$. They are

$$
S\left(\operatorname{Ra}^{224}\right)=0.34 \mathrm{e}^{-0.0011 t}
$$

and (2)

$$
S\left(\operatorname{Th}^{228}\right)=0.32 e^{-0.0010 t}
$$

The average weight of the spleens was $29.5 \pm 11.0 . g$ with a range of 10.7 to $47.0 \mathrm{~g}(?)$, so that $\mathrm{Ra}^{224}$ concentration in plasma relative to that in spleen is

$$
\frac{5.6 \times 10^{-4}}{0.34 / 29.5}=0.053
$$

In all cases the activity of $\mathrm{Pb}^{212}$ was less than that of $\mathrm{Ra}^{224}$ which means that either or both $\mathrm{Tn}^{220}$ and $\mathrm{Pb}^{212}$ are being moved out of the spleen. The $\mathrm{Pb}^{212}$ activity was less than that of $\mathrm{Th}^{228}$ in all but 2 spleens. The data can be described approximately by the equation

$$
\mathrm{S}\left(\mathrm{Pb}^{212}\right)=0.16 \mathrm{e}^{-0.00091 t}
$$

In the dog the spleen is a storage site for red blood cells (9), and this is probably a factor in the great range of values observed for both weight of and activity of $\mathrm{Pb}^{212}$ in the spleen. The concentration of $\mathrm{Pb}^{212}$ in the spleen ranged from 0.28 to 1.5 times that in blood cells (Table VII). Further, the bone marrow of all these dogs had received considerable irradiation and it is likely that they had to draw on their 

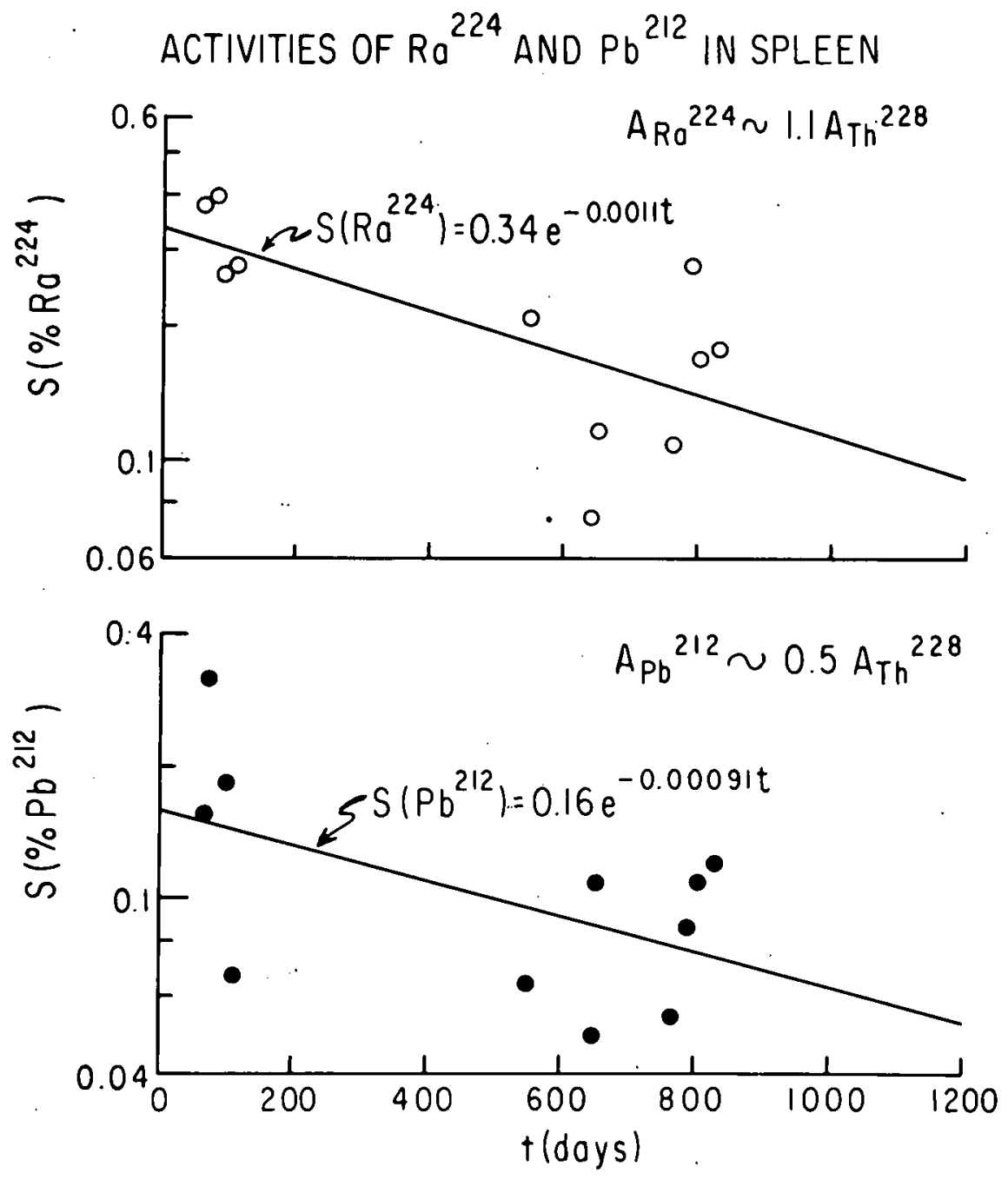

Fig. 8. Activities of $\mathrm{Ra}^{224}$ and $\mathrm{Pb}^{212}$ in spleen. 
TABLE VII

Activities of $\mathrm{Th}^{228}, \mathrm{Ra}^{224}, \mathrm{~Pb}^{212}$ and $\mathrm{Bi}^{212}$

in Spleen and Kidneys

\begin{tabular}{|c|c|c|c|c|c|c|c|c|c|}
\hline \multirow[b]{2}{*}{ Dog } & \multirow{2}{*}{$\begin{array}{l}\text { Days } \\
\text { After } \\
\text { Injection }\end{array}$} & \multicolumn{3}{|c|}{ Spleen } & \multirow{2}{*}{$\frac{\mathrm{Pb}^{212} / \mathrm{g} \text { spleen }}{\mathrm{Pb}^{212} / \mathrm{g} \text { b. cells }}$} & \multicolumn{4}{|c|}{ Kidneys } \\
\hline & & $\operatorname{Th}^{228}$ & $\mathrm{Ra}^{224}$ & $\mathrm{~Pb}^{212}$ & & $\mathrm{Th}^{228}$ & $\mathrm{Ra}^{224}$ & $\mathrm{~Pb}^{212}$ & $B i^{212}$ \\
\hline T5T5 & 65 & 0.27 & 0.38 & 0.15 & 0.44 & 1.09 & 0.29 & 0.34 & 2.13 \\
\hline M4T2 & 78 & 0.39 & 0.39 & 0.32 & - & 2.00 & 0.59 & 0.54 & 1.07 \\
\hline M2T5 & 97 & 0.38 & 0.27 & 0.18 & 0.41 & 0.98 & 0.36 & 0.30 & 1.36 \\
\hline T4T5 & 113 & 0.20 & 0.28 & 0.07 & 0.79 & 0.98 & 0.24 & 0.21 & -- \\
\hline MlT5 & 212 & -- & -- & -- & -- & 1.06 & 0.30 & 0.08 & -- \\
\hline F3T3 & 547 & 0.30 & 0.21 & 0.06 & 0.60 & 1.03 & 0.35 & 0.48 & 2.48 \\
\hline MlT4 & 645 & 0.05 & 0.07 & 0.05 & 0.38 & 0.29 & 0.10 & 0.13 & 0.58 \\
\hline $\mathrm{T} 6 \mathrm{~T} 4$ & 651 & 0.13 & 0.11 & $0: 06$ & 0.28 & 0.82 & 0.20 & -- & 0.58 \\
\hline F3T4 & 763 & 0.11 & 0.11 & 0.05 & 1.5 & 1.69 & 0.35 & 0.16 & -- \\
\hline $\mathrm{M} 4 \mathrm{~T} 4$ & 793 & 0.17 & 0.27 & 0.09 & 0.48 & 0.74 & 0.16 & -- & 0.29 \\
\hline M4T3 & 801 & 0.17 & 0.17 & 0.11 & 0.65 & 0.73 & 0.19 & 0.42 & 1.43 \\
\hline M2T4 & 833 & 0.10 & 0.18 & 0.12 & 0.71 & 0.64 & 0.15 & 0.10 & 0.22 \\
\hline MIT3 & 988 & 0.06 & -- & -- & -- & 0.82 & 0.36 & 0.37 & 0.51 \\
\hline
\end{tabular}

splenic reserves (10). This. suggests that at earlier times and/or lower dose levels the $\% \mathrm{~Pb}^{212}$ in spleen would be higher.

Kidneys. The activity of $\mathrm{Ra}^{224}$ was determined in the kidneys of 13 dogs, that of $\mathrm{Pb}^{212}$ in 11 , and that of $\mathrm{Bi}^{212}$ in 10 . The results appear in Table VII and Fig. 9. When descending exponentials were calculated for each of the 3 sets of data the coefficients of $t$ in the exponential did not differ significantly from zero. A similar result was obtained for $\mathrm{Th}^{228}$ (2). The mean values from Table VII are

$$
\begin{aligned}
& \mathrm{K}\left(\mathrm{Th}^{228}\right)=0.99 \pm 0.44 \\
& \mathrm{~K}\left(\mathrm{Ra}^{224}\right)=0.28 \pm 0.13 \\
& \mathrm{~K}\left(\mathrm{~Pb}^{212}\right)=0.28 \pm 0.16 \\
& \mathrm{~K}\left(\mathrm{Bi}^{212}\right)=1.16 \pm 0.76
\end{aligned}
$$


Activities of $\mathrm{Ro}^{224}, \mathrm{~Pb}^{212}$, and $\mathrm{Bi}^{212}$, in Kidneys

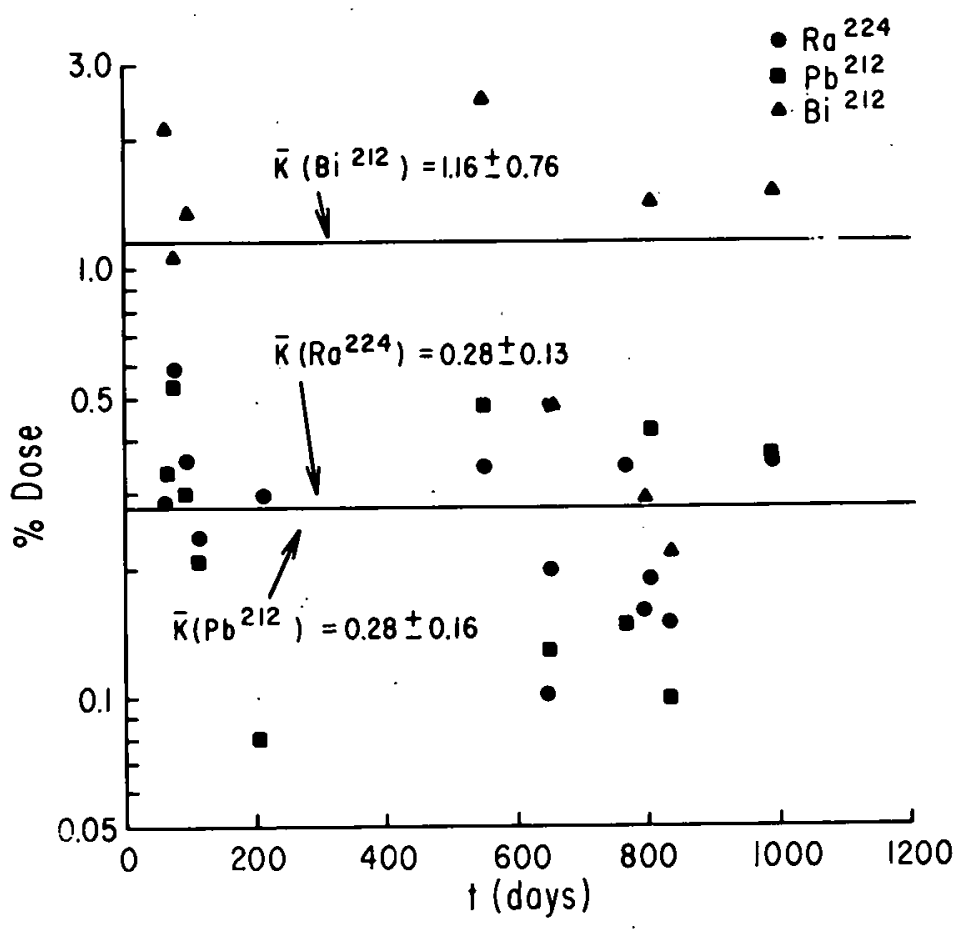

Fig. 9. The activities of $\mathrm{Ra}^{224}, \mathrm{~Pb}^{212}$, and $\mathrm{Bi}^{212}$ in kidneys. 
The activity of $\mathrm{Ra}^{224}$ was less than that of $\mathrm{Th}^{228}$ in all cases iñcluding MIT5 which suffered severe kidney damage. The average of the ratios of $\mathrm{Ra}^{224}$ to $\mathrm{Th}^{228}$ activity was $0.29 \pm 0.07$, while the ratio of the averages was $0.28 \pm 0.18$. Thus there is more consistency in the activity of $\mathrm{Ra}^{224}$ relative to the activity of $\mathrm{Th}^{228}$ and more variation in \% $\mathrm{Ra}^{224}$ and $\% \mathrm{Th}^{228}$.

From Eqs. (57) and (58) and the cumulative urinary excretion of $\mathrm{Ra}^{224}$ formed in the dog, Eqs. (28) and (32) it can be shown that the kidneys are clearing $\mathrm{Ra}^{224}$ from the blood. If $\mathrm{Ra}^{224}$ were not being moved to the kidneys from other parts of the dog then $\Sigma U+K\left(\mathrm{Ra}^{224}\right)$ would be equal to $0.99 \pm 0.44, \mathrm{Eq} \cdot(57)$, i.e. $\mathrm{Th}^{228}$ decaying in the kidneys would be the sole source. However, at $t=100$ days $\Sigma U+K\left(\mathrm{Ra}^{224}\right)$ $=3.7$ and $3.7-0.99=2.7 \% \mathrm{Ra}^{224}$ is removed from other parts of the animal. At 500 days, $\Sigma U+K\left(\mathrm{Ra}^{224}\right)=2.7$, still well in excess of 0.99 \pm 0.44 .

Of the 4 nuclides, $\mathrm{Pb}^{212}$ was the most difficult to determine. Success depended on having sufficient points during the short period which begins when $\mathrm{Bi}^{212}$ has decayed to transient equilibrium with $\mathrm{Pb}^{212}$ and ends when $\mathrm{Pb}^{212}$ reaches transient equilibrium with $\mathrm{Ra}^{224}$. In all 11 kidneys the activity of $\mathrm{Pb}^{212}$ was less than that of $\mathrm{Th}^{228}$, and in 6 it was less than that of $\mathrm{Ra}^{224}$ and higher in 5 , with the mean values about the same, Eqs. (58) and (59).

Next we relate some of the $\mathrm{Pb}^{212}$ findings. At 778 days $0.4 \% \mathrm{~Pb}^{212}$. $\left(0.23 \% \mathrm{~Pb}^{212} / \mathrm{d}\right)$ was decaying outside dog T8T3 via urinary excretion. Since the activities of $\mathrm{Ra}^{224}$ and $\mathrm{Pb}^{212}$ in the kidneys are about the same, $\mathrm{Pb}^{212}$ is being brought to the kidneys by the blood. At 784 days 
T8T3 had $0.0065 \% \mathrm{~Pb}^{212} / \mathrm{g}$. blood cells (compare with the 784 day value of 0.0051 from Eq. (6)). Thus; the effective clearance is

$$
\frac{0.23 \% \mathrm{~Pb}^{212} / \mathrm{d}}{0.0065 \% \mathrm{~Pb}^{212} / \mathrm{g} \mathrm{blood} \mathrm{cells}}=\frac{35 \mathrm{~g} \text { cells }}{\text { day }}
$$

From the studies in which beagle blood cells were tagged with $\mathrm{Pb}^{212}$ in

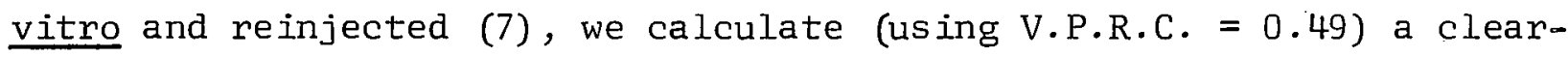
ance of $31 \mathrm{~g}$ blood cells/day.

Dog T8T3 weighed $7.5 \mathrm{~kg}$, and using $43 \mathrm{ml}$ cells $/ \mathrm{kg}$ dog (10) and 1.09 g cells/ml cells (ll) we calculate

$$
0.0065 \times 1.09 \times 43.0 \times 7.50=2.3 \% \mathrm{~Pb}^{212}
$$

for the steady state activity of $\mathrm{Pb}^{212}$ associated with circulating blood cells.

Since $\mathrm{Bi}^{212}$ has a short half period ( 1 hour), its activity at time of death can be determined only if counting is begun soon after death. Also, the determination is facilitated when the activity of $\mathrm{Bi}^{212}$ is high relative to that of $\mathrm{Pb}^{212}$. This injects some bias into the results. Counting of the 10 kidneys for which there arc $\mathrm{Bi}^{212}$ entries in Table VII was begun at a mean time of 1.3 hours after death (range 0.8 to 2.2 hours). The times for the other 3 were $1.6,2.5$, and 2.0 hours. It is likely that the $\mathrm{Bi}^{212}$ activity in each of these 3 was greater than $\mathrm{Pb}^{212}$ but less than the average, Eq. (60), so that the average given is slightly high for the set of 13 kidneys. In 4 of 10 kidneys $\mathrm{Bi}^{212}$ activity 
was lower than that of $\operatorname{Th}^{228}$ and it was higher in 6 . In all cases where determination was possible the activity of $\mathrm{Bi}^{212}$ exceeded that of $\mathrm{Ra}^{224}$ and of $\mathrm{Pb}^{212}$. Thus $\mathrm{Bi}^{212}$ is being moved to the kidneys from other parts of the animal.

Dog T8T3 provides suggestive evidence that the combined activity of $\mathrm{Bi}^{212}$ in kidneys and urine is greater than that expected from $\mathrm{Bi}^{212}$ formed in the kidney and $\mathrm{Bi}^{212}$ formed in or on and escaping from blood cells. The measured steady state activity of $\mathrm{Bi}^{212}$ in urine was 0.45 $0.91 \% \mathrm{Bi}^{212}$, Eq. (49). Using this value plus the average kidney $\mathrm{Bi}^{212}$ activity of Eq. (60) we estimate the total in urine and kidneys at 1.6 to $2.1 \% \mathrm{Bi}^{212}$. By Eq. (59) we expect formation of $\mathrm{Bi}^{212}$ in kidneys to be about $0.3 \%$. Associated with the blood cells is $2.3 \% \mathrm{~Pb}^{212}$, Eq. (62) . One early count was obtained from this sample (at 42 mins. after this blood was drawn). From this one point the calculated $B i^{212}$ activity in blood cells was 1.03 that of $\mathrm{Pb}^{212}$. Hence little or no $\mathrm{Bi}^{212}$ was moving out of the cells so that the cells were not a significant source of the $\mathrm{Bi}^{212}$ in kidneys and urine. Thus we conclude that $\mathrm{Bi}^{212}$ is moved to the kidneys and urine from other (not blood cells) sites in the dog by way of the plasma.

The following related study gives further support to this conclusion. A 7 year old female beagle, F54A, was anesthetized with nembutol, a urinary catheter was introduced, and the dog was given an intravenous injection of $11.1 \mu \mathrm{C} \mathrm{Pb}{ }^{212}$ with $\mathrm{Bi}^{212}$ in transient equilibrium in citrate buffer $\mathrm{pH} \simeq 3.5,0.08 \mathrm{M}$ total citrate. Blood was drawn at $3.4 \mathrm{~min}$. and at hourly intervals for 6 hours. Urine was obtained, via the catheter, at hourly intervals for 6 hours. The anesthesia was maintained for the 
duration of the experiment. At 1 hour after injection $100 \mathrm{ml}$ normal saline was given subcutaneously. After the final urine was withdrawn, the bladder was washed with $10 \mathrm{ml}$ saline which was then added to the urine sample. $\mathrm{Pb}^{212}$ and $\mathrm{Bi}^{212}$ activities were measured in the various plasma, blood cell, and urine samples and the results appear in Table VIII.

\section{TABLE VIII}

$\mathrm{Pb}^{212}$ and $\mathrm{Bi}^{212}$ in Blood Cells and Plasma and in Urine Obtained by Catheterization Following Intravenous Injection of $\mathrm{Pb}^{2 \mathrm{~L} 2}$ and $\mathrm{Bi}^{212}$ in Transient Equilibrium

\begin{tabular}{|c|c|c|c|c|c|c|}
\hline \multirow[b]{2}{*}{$t(\mathrm{~min})$} & \multicolumn{3}{|c|}{ Plasma } & \multicolumn{3}{|c|}{ Cells } \\
\hline & & $\% \mathrm{~Pb}^{212} / \mathrm{g}$ & $\% \mathrm{Bi}{ }^{212} / \mathrm{g}$ & $\% \mathrm{~Pb}^{212} / \mathrm{g}$ & $\% \mathrm{Bi}^{212} / \mathrm{g}$ & $\mathrm{Bi}^{212} / \mathrm{Pb}^{212}$ \\
\hline 3.4 & & 0.012 & 0.069 & 0.089 & 0.020 & 0.22 \\
\hline 61.1 & & 0.002 & 0.009 & 0.113 & 0.041 & 0.36 \\
\hline 118 & $\leq$ & 0.001 & 0.006 & 0.119 & 0.072 & 0.60 \\
\hline 179 & $\leq$ & 0.001 & 0.005 & 0.118 & 0.092 & 0.78 \\
\hline 243 & $\leq$ & 0.001 & 0.003 & 0.119 & 0.091 & 0.77 \\
\hline 305 & $\leq$ & 0.001 & 0.004 & 0.115 & 0.099 & 0.86 \\
\hline 365 & $\leq$ & 0.001 & 0.004 & 0.114 & 0.109 & 0.93 \\
\hline
\end{tabular}

\begin{tabular}{|c|c|c|c|c|c|c|}
\hline \multicolumn{7}{|c|}{ Urine } \\
\hline$t(\min )$ & $\% \mathrm{~Pb}^{212}$ & $\begin{array}{l}\text { Cum. } \\
\% \mathrm{~Pb}^{212}\end{array}$ & $\% B i^{212}$ & Cum. & $\frac{\text { Cum. } \% \mathrm{Bi}^{212}}{\% \mathrm{~Pb}^{212} / \mathrm{g} \mathrm{Cells}}$ & $\frac{\% \mathrm{Bi}^{212}}{\% \mathrm{~Pb}^{212}}$ \\
\hline 59 & 0.51 & 0.51 & 2.25 & 2.25 & 25 & 4.4 \\
\hline 116 & 0.67 & 1.18 & 2.44 & 3.68 & 32 & 3.6 \\
\hline 178 & 0.76 & 1.94 & 2.37 & 4.30 & 36 & 3.1 \\
\hline 240 & 0.35 & 2.29 & 1.33 & 3.59 & 30 & 3.8 \\
\hline 300 & 0.14 & 2.43 & 0.71 & 2.62 & 23 & 5.0 \\
\hline 360 & 0.19 & 2.62 & 0.85 & 2.25 & 20 & 4.6 \\
\hline
\end{tabular}

Before considering the results it should be noted that the study has 3 limitations with respect to using the results to help understand 
the migrations of the Th ${ }^{228}$ decay products. First is the unknown effect of the 6 hour anesthesia; second the dog was 7.1 years old whereas the $\mathrm{Th}^{228}$ dogs were roughly 1.5 to 5 years (T8T3 was 3.8 years at 778 days after injection); third, the dog was purchased from an outside kennel 3 weeks prior to the experiment and there was no medical record available to indicate whether there had been diseases which could have led to impairment of kidney function.

A measured aliquot of the injection standard was counted alternately with the samples and the data were analyzed in terms of ratios of sample to standard count rates. This method removes the $\mathrm{Pb}^{212}$ decay factor and also effects the decay constant of $\mathrm{Bi}^{212}$ insofar as determining its growth and decay in sets of samples is concerned. The advantage of the method is that the biological rates can be examined more easily if the effect of physical decay is removed.

Let subscripts 1 and 2 denote $\mathrm{Pb}^{212}$ and $\mathrm{Bi}^{212}$, and $\mathrm{s}$ and $\mathrm{S}$ the sample and standard count rates, respectively. The activities in transient equilibrium are given by

$$
\begin{gathered}
A_{1}=A_{1}^{0} e^{-\lambda_{1} t} \\
A_{2}=\frac{\lambda_{2}}{\lambda_{2}-\lambda_{1}} A_{1}^{0}\left(e^{-\lambda_{2} t}-e^{-\lambda_{2} t}\right)+A_{2}^{0} e^{-\lambda_{2} t} \\
A_{2}^{o}=\frac{\lambda_{2}}{\lambda_{2}-\lambda_{1}} A_{1}=1.11 A_{1}^{0} .
\end{gathered}
$$

Remembering that the detector essentially measures only $\mathrm{Bi}^{212}$ the 
standard count rate is

$$
s=c\left\{1.11 A_{1}^{o}\left(e^{-\lambda_{1} t}-e^{-\lambda_{2} t}\right)\right\}+A_{2}^{o} e^{-\lambda_{2} t}=c A_{2}^{o} e^{-\lambda_{1} t}
$$

where $\mathrm{c}$ is a constant including detection efficiency and dilution correction. The count rate of a sample collected at $t=T$ is, for $t \geq$ $\mathrm{T}$,

$$
s=c^{\prime}\left\{x \operatorname{lili} A_{1}^{T}\left(e^{-\lambda_{1}(t-T)}-e^{-\lambda_{2}(t-T)}\right)\right\}+y A_{2}^{T} e^{-\lambda_{2}(t-T)}
$$

where $x$ and $y$ are fractions of the $\mathrm{Pb}^{212}$ and $\mathrm{Bi}^{212}$ at $t=\mathrm{T}$. The sample to standard count rate ratio is then

$$
\begin{aligned}
\frac{s}{S}= & \frac{c^{\prime} A_{2}^{T}\left(x e^{-\lambda_{1}(t-T)}+(y-x) e^{-\lambda_{2}(t-T)}\right.}{c A_{2}^{O} e^{-\lambda_{1} t}} \\
& =\frac{c^{\prime}}{c}\left\{x+(y-x) e^{-\left(\lambda_{2}-\lambda_{1}\right)(t-T)}\right\}
\end{aligned}
$$

since $A_{2}^{o} e^{-\lambda_{1} t}=A_{2}^{T} e^{-\lambda_{1}(t-T)}$. Thus we have

$$
\% \mathrm{~Pb}^{21.2}=\left(\frac{c^{\prime}}{c} x\right) 100
$$

and

$$
\% \mathrm{Bi}^{212}=\left(\frac{\mathrm{c}^{\prime}}{\mathrm{c}} \mathrm{y}\right) 100
$$

Note that $c^{\prime} / c$ is just a ratio of dilution corrections. As seen in Table VIII, $\mathrm{Pb}^{212}$ concentration in blood cells reached a 
peak at about 2 hours, very much the same as in Ref. (7) and Fig. 2 whereas plasma concentration decreased rapidly to less than $1 \%$ that of cells. \% $\mathrm{Bi}^{212} / \mathrm{g}$ in plasma decreased too but was always higher than the $\% \mathrm{~Pb}^{212} / \mathrm{g}$.plasma. The concentration of $\mathrm{Bi}^{212}$ in cells increased throughout the course of the experiment. If $\mathrm{Bi}^{212}$ is associated with cells only if it is formed in cells, the ratio of activities is

$$
\frac{\% \mathrm{Bi}^{212}}{\% \mathrm{~Pb}^{212}}=a\left(1-e^{-\left(\lambda_{\mathrm{Bi}}{ }^{212}-\lambda_{\mathrm{Pb}}{ }^{212}\right) \mathrm{t}}\right)
$$

where $\mathrm{a}$ is fractional retention of $\mathrm{Bi}^{212}$ by cells. If no $\mathrm{Bi}{ }^{212}$ formed in cells is lost from cells, then $a=1$. Eq. (71) with $a=1$ is shown as the solid curve in Fig. 10. The least squares principle was used to calculate the asymptote from the observed $\mathrm{Bi}^{212} / \mathrm{Pb}^{212}$ ratios $(3.4 \mathrm{~min}$. point excluded) and yielded

$$
\mathrm{a}=0.916
$$

Thus $90 \%$ or more of the $\mathrm{Bi}^{212}$ formed in cells decays there.

From the peak concentration of $\mathrm{Pb}^{212}$ in cells the estimated total blood cell mass is (2/3) $100 \% / 0.12 \% / \mathrm{g}=555 \mathrm{~g}$ cells (7). The blood cell volume estimated from dog weight is $13 \mathrm{~kg} / .43 \mathrm{ml} / \mathrm{kg}=560 \mathrm{ml}$ cells (10). The activities of $\mathrm{Pb}^{212}$ and $\mathrm{Bi}^{212}$ in each urine sample at time of collection are given in Table VIII. Since the $\mathrm{Pb}^{212}$ decay factor has been taken out, the cumulative $\mathrm{Pb}^{212}$ is obtained by simply adding and $\mathrm{Bi}^{212}$ is accumulated using $\mathrm{e}^{-\left(\lambda_{\mathrm{Bi}}{ }^{212}-\lambda_{\mathrm{Pb}}{ }^{212)} \mathrm{t}\right.}$. At 6 hours the cumulative urinary excretion of $\mathrm{Pb}^{212}$ is $2.6 \%$ and the clearance is roughly 

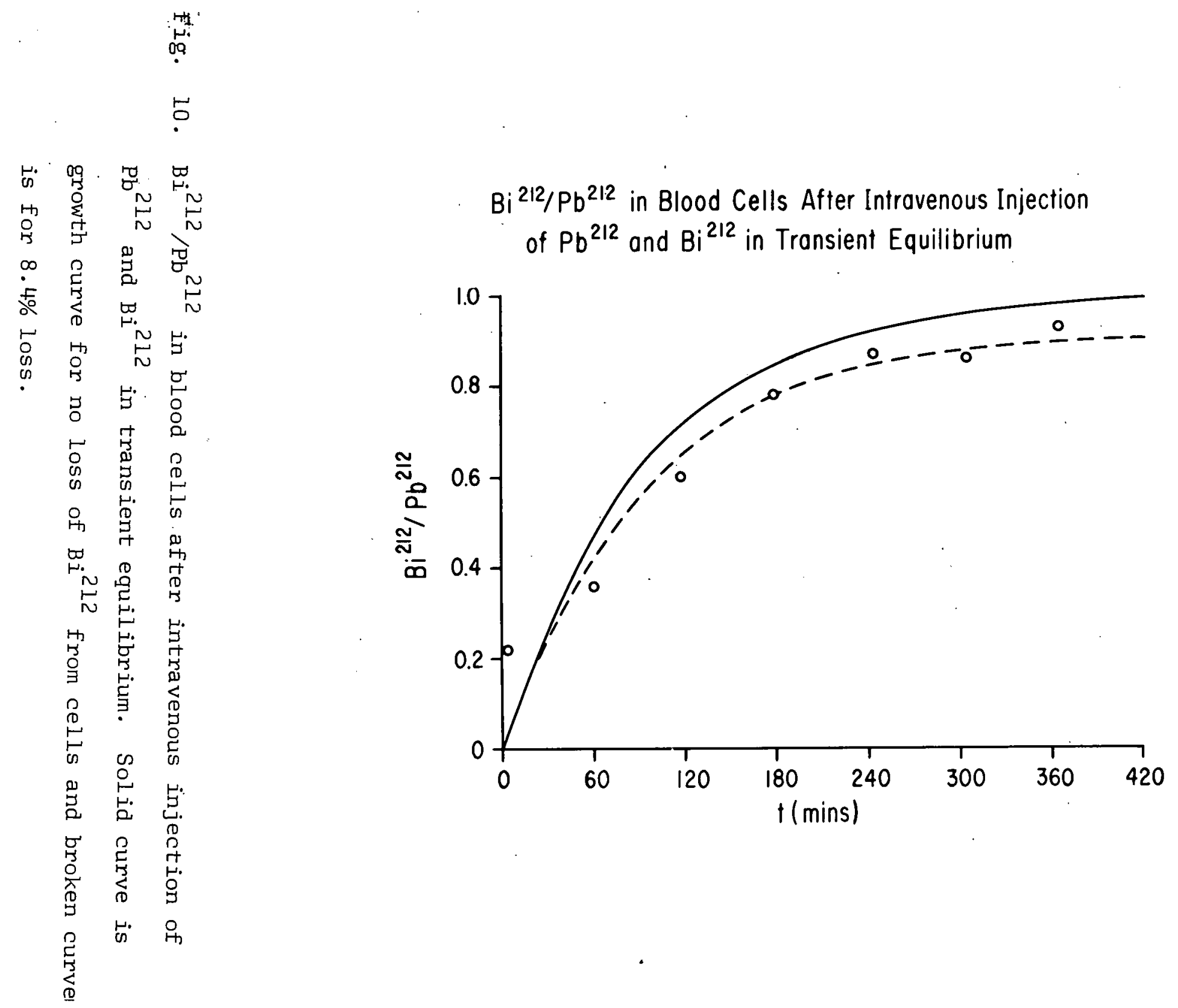
1 - 2 g cells/hour.

The 5 and 6 hour values for cumulative $\mathrm{Bi}^{212}$ urinary excretion are essentially steady state values. They are 2.6 and $2.2 \% \mathrm{Bi}^{212}$, respectively, and are equal to the activities of $\mathrm{Bi}^{212}$ being produced in 23 and $20 . \mathrm{g}$ blood cells. Thus the steady state urinary $\mathrm{Bi}^{212}$ is about (23/ 550) $100=4 \%$ of the $\mathrm{Bi}^{212}$ formed in blood cells and about $1 / 2$ of the $\mathrm{Bi}^{212}$ lost from these cells. If the cells lose $\mathrm{Bi}^{212}$ only to the kidneys, then the effective transit time from cells through the kidneys to the bladder is about 1 hour, i.e. $1 \mathrm{Bi}^{212}$ half-period.

The last column in Table VIII gives the ratios, $\% \mathrm{Bi}^{212} / \% \mathrm{~Pb}^{212}$, for each urine sample.

- Next we apply these results to the $\mathrm{Th}^{228}$ decay product studies. First these results show at least $90 \%$ of the $\mathrm{Bi}^{212}$ formed in blood cells decays there. Hence, blood cells are not the principal source of the relatively high activities of $\mathrm{Bi}^{212}$ found in kidneys of dogs given $\mathrm{Th}^{228}$. Second, the $\mathrm{Bi}^{212} / \mathrm{Pb}^{212}$ activity ratio in urine was considerably lower than in the urine samples from T8T3 described above. Since these urines were obtained by catheter a higher ratio would be expected because there was a shorter period for $\mathrm{Bi}^{212}$ decay between the time the urine left the kidneys and sample collection time.

Both of these factors provide additional evidence that some $\mathrm{Bi}^{212}$. formed at sites other than blood cells and kidneys is being moved to the kidneys. We have no indication of which sites are being depleted, although it does seem likely that they would be highly vasculari...

Humerus and Third Lumbar Vertebra. The activities of $\mathrm{Ra}^{224}$ and $\mathrm{Pb}^{212}$ in 15 humeri, of $\mathrm{Ra}^{224}$ in 13 and $\mathrm{Pb}^{212}$ in 12 third lumbar vertebrae 
have been measured. The results are shown in Fig. 1l. In all of the humeri both $\mathrm{Ra}^{224}$ and $\mathrm{Pb}^{212}$ activities were less than that of $\mathrm{Th}^{228}$, and $\mathrm{Pb}^{212}$ activity was less than that of $\mathrm{Ra}^{224}$ except in one case in which they were nearly equal. In one vertebra the activities of $\mathrm{Th}^{228}, \mathrm{Ra}^{224}$, and $\mathrm{Pb}^{212}$ were equal, in two others $\mathrm{Pb}^{212}$ and $\mathrm{Ra}^{224}$ activities were

Activities of $\mathrm{Ra}^{224}$ and $\mathrm{Pb}^{212}$ in One Humerus $(\mathrm{H})$ and The Thi. Lumbor Vertebro. (V)

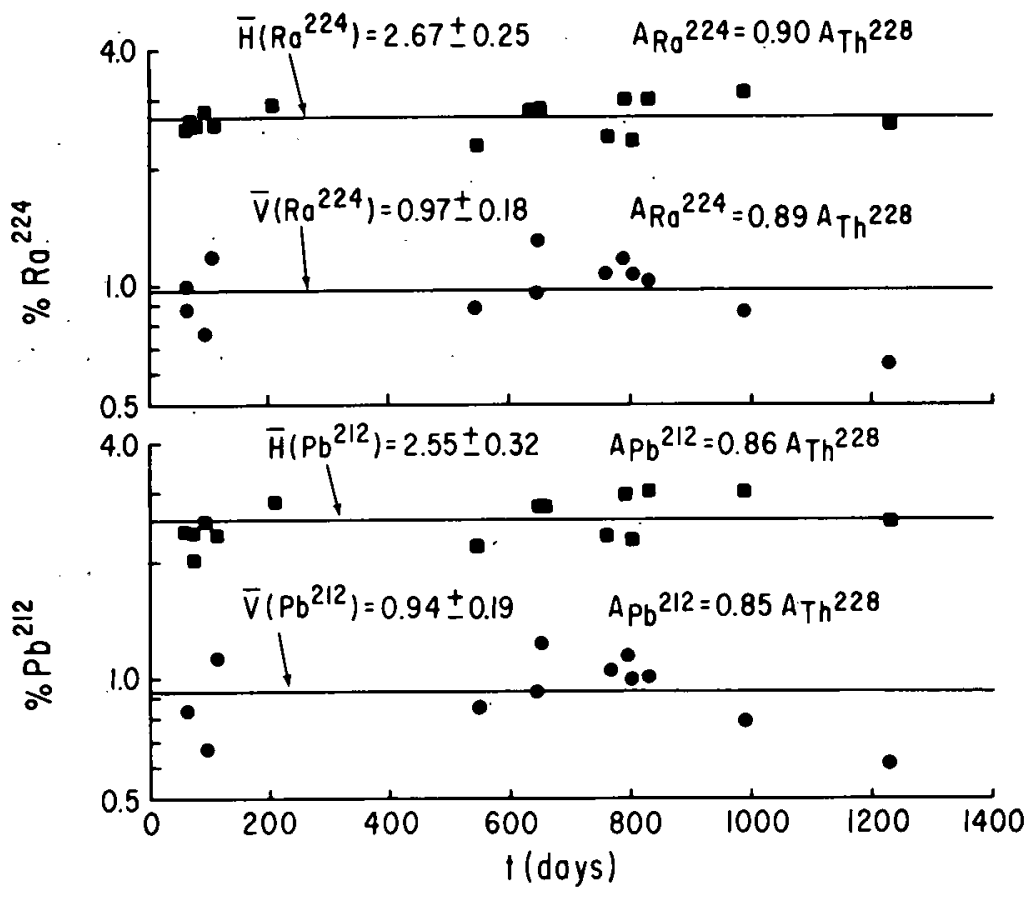

Fig. 11. The activities of $\mathrm{Ra}^{224}$ and $\mathrm{Pb}^{212}$ in one humerus and the third lumbar vertebra. 
equal. In the others the activity of $\mathrm{Pb}^{212}$ was less than that of $\mathrm{Ra}^{224}$ which was less than that of $\mathrm{Th}^{228}$.

The data were analyzed for a change with time, as described above, but there was no significant change for either nuclide in either bone. The sums of the activities in the two bones, $\mathrm{H}+\mathrm{V}$, and the several activity ratios in each bone, $\mathrm{H}$ and $\mathrm{V}$, and the sum, $\mathrm{H}+\mathrm{V}$, also did not show a significant change with time. The means and standard deviations of these various quantities are given in Table IX, which also includes the previously reported measurements of $\mathrm{Th}^{228}$ (2).

\section{TABLE IX}

Activities of $\mathrm{Ra}^{224}$ and $\mathrm{Pb}^{212}$ in One Humerus

$(\mathrm{H})$ and the Third Lumbar Vertebra (V)

\begin{tabular}{lcccccc}
\hline Quantity $^{\mathrm{a}}$ & $\mathrm{n}$ & $\overline{\mathrm{H}}$ & $\mathrm{n}$ & $\overline{\mathrm{V}}$ & $\mathrm{n}$ & $\overline{(\mathrm{H}+\mathrm{V})}$ \\
\hline$\% \mathrm{Ra}^{224}$ & 15 & $2.67 \pm 0.25$ & 13 & $0.972 \pm 0.179$ & 13 & $3.64 \pm 0.32$ \\
$\mathrm{Ra}^{224} / \mathrm{Th}^{228}$ & 15 & $0.904 \pm 0.052$ & 13 & $0.892 \pm 0.074$ & 13 & $0.895 \pm 0.054$ \\
$\% \mathrm{~Pb}^{212}$ & 15 & $2.55 \pm 0.32$ & 12 & $0.940 \pm 0.193$ & 12 & $3.53 \pm 0.38$ \\
$\mathrm{~Pb}^{212} / \mathrm{Th}^{228}$ & 15 & $0.862 \pm 0.076$ & 12 & $0.854 \pm 0.098$ & 12 & $0.866 \pm 0.070$ \\
$\mathrm{~Pb}^{212} / \mathrm{Ra}^{224}$ & 15 & $0.954 \pm 0.058$ & 12 & $0.965 \pm 0.032$ & 12 & $0.967 \pm 0.029$ \\
$\% \mathrm{Th}^{228^{b}}$ & 21 & $2.86 \pm 0.45$ & 20 & $1.2 \mathrm{e}^{-0.00025 t}$ & 20 & $4.2 \mathrm{e}^{-0.00014 t}$.
\end{tabular}.

a Mean and standard deviation are given.

b From Ref。 (2).

These two bones contained $6.1 \%$ of the $\operatorname{Th}^{228}$ in the skeleton and this was used to calculate the equation for $\operatorname{Th}^{228}$ in the skeleton: 


$$
B\left(\operatorname{Th}^{228}\right)=69 e^{-0.00014 t}
$$

If we assume that the $\mathrm{Ra}^{224} / \mathrm{Th}^{228}$ ratio in $\mathrm{H}+\mathrm{V}$ is representative of the entire skeleton, then these two bones contain $6.1 \%$ of the $\mathrm{Ra}^{224}$ in the skeleton, and the equation for total $\mathrm{Ra}^{224}$ in bone is

$$
B\left(\operatorname{Ra}^{224}\right)=0.895 B\left(\mathrm{Th}^{228}\right)=62 \mathrm{e}^{-0.00014 t}
$$

The latter assumption seems valid since the major part of the $\mathrm{Ra}^{224}$ does not translocate at times greater than 100 days. The counter argument is that $\% \mathrm{Ra}^{224}$ /bone did not decrease with time by the above mentioned criteria. Similarly we estimate the total $\mathrm{Pb}^{212}$ in the skeleton to be

$$
\begin{gathered}
\mathrm{B}\left(\mathrm{Pb}^{212}\right)=0.967 \mathrm{~B}\left(\mathrm{Ra}^{224}\right)=0.866 \mathrm{~B}\left(\mathrm{Th}^{228}\right) \\
=60 \mathrm{e}^{-0.00014 t}
\end{gathered}
$$

Balancing the Books. From excretion studies we have determined the $\% \mathrm{Ra}^{224}$ which decays in the dog, and from measurement of selected bones and soft tissues we have determined the $\% \mathrm{Ra}^{224}$. which decays at these sites. Are the results from the two kinds of measurements compatible? Fig. 12A. provides a graphical comparison. At the top is plotted the retention equation, Eq. (35). The bottom curve is the estimate of $\mathrm{Ra}^{224}$ in bone, Eq. (74), and above it is the sum of the $\mathrm{Ra}^{224}$ in bone, liver, spleen, and kidneys, Eqs. $\left(74,52,54\right.$, and 58). The total $\mathrm{Ra}^{224}$ in plasma is small compared with this sum (about $0.25 \%$ at 100 days and about 
$0.08 \%$ at 1000 days) and was not included. There is one more site about which we have some information; it is the intestinal tract. If we guess

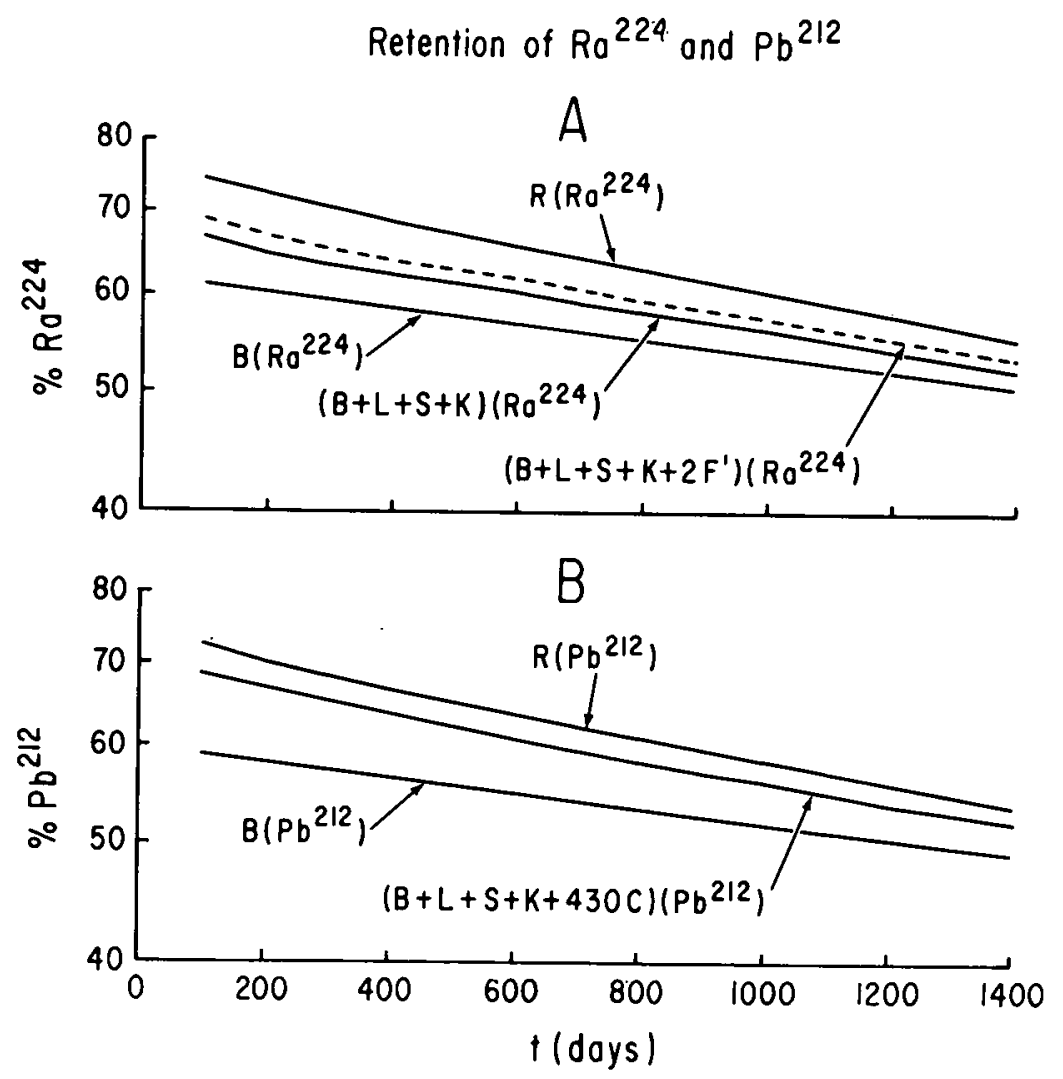

Fig. 12. A. Comparison of the retention of $\mathrm{Ra}^{224}, \mathrm{R}$, with the $\mathrm{Ra} 224$ in bone, $\mathrm{B}$, with the total $\mathrm{Ra}^{224}$ in bone, liver, spleen, and kidneys, $\mathrm{B}+\mathrm{L}+\mathrm{S}+\mathrm{K}$, and with that total plus the estimated $\mathrm{Ra}^{224}$ in the intestinal tract, $2 \mathrm{~F}^{\prime} \cdot \mathrm{B}$. Comparison of the retention of $\mathrm{Pb}^{212}$, $\mathrm{R}$, with the $\mathrm{Pb}^{212}$ in bone, $\mathrm{B}$, and with the total $\mathrm{Pb}^{212}$ in bone, liver, spleen, kidneys, and blood cells, $B+L+S+K+430 C$. 
that the intestinal tract contains twice the daily fecal excretion of $\operatorname{Ra}^{224}$ and add $2 \mathrm{~F}^{\prime}$, ffrom Eq. (20) to the above sum we obtain the broken curve of Fig. 12A. This curve is just a little lower than the retention curve, which is appropriate for at least two reasons. First, there undoubtedly is some $\mathrm{Ra}^{224}$. in the other soft tissues which were not measured, and, secorid, retention derived from excretion is more likely to be high than low.

In Fig. 12B. a similar comparison of the $\mathrm{Pb}^{212}$ results has been made. The top curve is the retention equation, Eq. (48). The bottom curve is the estimate of $\mathrm{Pb}^{212}$ in bone, $\mathrm{Eq} \cdot(75)$. The middle curve is the sum of the $\mathrm{Pb}^{212}$ in bone, liver, spleen, kidneys, and blood cells, from Eqs. (75, 53, 56, 59 and 6). . The total weight of blood cells used is

$$
\frac{43 \mathrm{ml}}{\mathrm{kg}} \times 9.2 \mathrm{~kg} \times \frac{1.098}{\mathrm{ml}}=430 \mathrm{~g} \text { cells. }
$$

Again the sum curve is seen to differ appropriately from the retention curve. Thus the results of the two kinds of measurements are consistent. Some Estimates of Average Radiation Dose Rates. The short-lived nuclide $\operatorname{Tn}^{220}$ presents a problem in estimating radiation dose rates since its half-period is too short to permit its measurement in tissue samples obtained at autopsy. From $\mathrm{Ra}^{224}$ retention, Eq. (36), we know how much $\operatorname{Tn}^{220}$ is formed in the dog and from the $\operatorname{Tn}^{220}$ exhalation studies (4) we know how much $\operatorname{Tn}^{220}$ decays in the dog, but we lack precise information on how much $\mathrm{Tn}^{220}$ decays' in tissues of interest.

Let us consider bone. The biological rate of decrease of $\operatorname{Th}^{228^{\prime}}$ in 
bone is slow with a half-period of about 14 years, Eq. (73) . Throughout the period studied the activity of $\mathrm{Ra}^{224}$ was less than that of $\mathrm{Th}^{228}$ showing that some $\mathrm{Ra}^{224}$ in bone moves out to decay elsewhere. Also the biological rate of decrease of $\mathrm{Ra}^{224}$ in bone is at least as slow as that of. $\mathrm{Th}^{228}$, and may be slower, Table IX. The activity of $\mathrm{Pb}^{212}$ in bone was less than that of $\mathrm{Ra}^{224}$. This means that either some $\mathrm{Tn}^{220}$ or some $\mathrm{Pb}^{212}$ or some of both nuclides move out of bone to decay elsewhere. There is the further possibility that some of the $\mathrm{Pb}^{212}$ found in bone was formed elsewhere in the dog and then deposited in bone. If bone were the only site of formation of $\operatorname{Tn}^{220}$ we could readily distinguish among these possibilities, but since there is appreciable $\mathrm{Ra}^{224}$ in soft tissues this is not possible.

However, we can estimate $\operatorname{Tn}^{220}$ activity in bone from several different sets of assumptions and make comparisons.

First we reject the possibility that $\operatorname{Tn}^{220}$ activity in bone is greater than that of $\mathrm{Ra}^{224}$. This possibility would require a transfer of $\operatorname{Tn}^{220}$ from soft tissues to bone which is incompatible with our experience with $\mathrm{Ra}^{224}$ formed in vivo and also with $\mathrm{Rn}^{22}$ formed in vivo.

Second we examine the possibility that the activities of $\operatorname{Tn}^{220}$ and $\mathrm{Ra}^{224}$ in bone are equal, i.e.

$$
B_{\max }\left(\operatorname{Tn}^{220}\right)=B\left(\operatorname{Ra}^{224}\right)=62 e^{-0.00014 t}
$$

Eq. (76) provides an upper limit for $\operatorname{Tn}^{220}$ in bone and it states that there is no net transfer of $\operatorname{Tn}^{220}$ from bone to blood and/or soft tissues, and, hence that the soft tissues are the source of the $\operatorname{Tn}^{220}$ which decays 
in blood and which is exhaled. Intuitively we expect that $\operatorname{Tn}^{220}$ would move more rapidly from a highly vascular soft tissue than from bone. If the transit time from soft tissue to blood is close to the $\operatorname{Tn}^{220}$ halfperiod and. the transit time from bone to blood is appreciably greater, then soft tissues will be depleted of $\operatorname{Tn}^{220}$ and bone will not.

Experimentally. we: know that there is ample $\mathrm{Ra}^{224}$ in soft tissues to supply the $\operatorname{Tn}^{220}$ which decays in blood or is exhaled. An additional clue comes from our studies of $\mathrm{Pb}^{212}$ associated with the blood cells. The principal source of this $\mathrm{Pb}^{212}$ is undoubtedly the $\mathrm{Tn}^{220}$ which decays in the blood with transfer of $\mathrm{Pb}^{212}$ from bone and soft tissues being a minor source. The biological rate of decrease of $\mathrm{Pb}^{212}$ in blood cells, from Eq. (4), must. be the same as that rate for its source. Comparison of Eqs. (4, 52, 54 and 74) shows that the biological rate of decrease of $\mathrm{Pb}^{212}$ in blood cells is essentially the same as the rate at which $\mathrm{Ra}^{224}$ in liver and spleen decreases, but it is about 7 times greater than the rate at which $\mathrm{Ra}^{224}$ in bone decreases. From the requirement of equivalent rates it follows that soft tissues are the source of most of the $\operatorname{Tn}^{220}$ going to the blood.

The thind possibility to examine is that all the $\operatorname{Tn}^{220}$ which decays in blood or is exhaled comes from bone and none comes from soft tissues. While this seems highly unlikely, it is worth considering since it provides a method of setting a lower limit on the activity of $\operatorname{Tn}^{220}$ in bone. Assume that $\mathrm{Pb}^{212}$ formed from $\mathrm{Tn}^{220}$ decay in the blood behaves as $\mathrm{Pb}^{212}$ given intravenously, i.e. that $52 \%$ decays in the blood (7). Also assume $\operatorname{Tn}^{220}$ decay in blood is the only source of the $\mathrm{Pb}^{212}$ associated with blood cells. Then we can calculate an upper limit for how much 
$\operatorname{Tn}^{220}$ (and $\mathrm{Po}^{214}$ ) decays in blood. It is

Max. $\% \operatorname{Tn}^{220}$ decaying in blood $=430 \mathrm{C} / 0.52=9.1 \mathrm{e}^{-0.00097 t}$

The minimum activity of $\operatorname{Tn}^{220}$ in bone is then the $\operatorname{Tn}^{220}$ formed in bone diminished by the $\operatorname{Tn}^{220}$ which decays in blood and which is exhaled. Using the estimate of Eq. (45) for the latter together with Eqs. (74) and (76) we have

$$
B_{\min }\left(\operatorname{Tn}^{220}\right)=62 e^{-0.00014 t}-9.1 e^{-0.00097 t}-2.5 e^{-0.0003 t}
$$

Next we calculate maximum and minimum average radiation dose rates to bone using Eqs. $\left(73,74,75,76\right.$, and 78) for $\mathrm{Th}^{228}, \mathrm{Ra}^{224}, \mathrm{~Pb}^{212}$, and $\operatorname{Tn}_{\max }^{220}$ and $\operatorname{Tn}_{\min }^{220}$, respectively. Early counts on bone samples showed that there was no, great difference between $\mathrm{Pb}^{212}$ and $\mathrm{Bi}^{212}$ activities. The studies of $\mathrm{Bi}^{212}$ in blood, kidneys, and urine presented above showed that 1 to $1.5 \% \mathrm{Bi}^{212}$ moves to kidneys and urine from other sites. We assume that this small amount of $\mathrm{Bi}^{212}$ does not come from bone, and that the activities of $\mathrm{Pb}^{212}, \mathrm{Bi}^{212}, \mathrm{Po}^{212}$, and $\mathrm{Tl}^{208}$ in bone are equal. Then we have, assuming skeletal weight $=0.1$ body weight and using average alpha, beta, and recoil energies (I),

$$
\begin{aligned}
& \frac{\mathrm{dD}_{\text {max }}}{\mathrm{dt}}=\{0.69 \times(5.50+0.62 \times 19.07+0.60 \times 8.70)\} \times \\
& e^{-0.00014 t} e^{-\lambda} \operatorname{Th}^{228 t} \times I \times 0.512 \\
& =10.6 \mathrm{e}^{-0.00114 t} \mathrm{I} \text { rads/day }
\end{aligned}
$$


where $\lambda_{\mathrm{Th}^{2}} 228=0.00100, \mathrm{I}=\mu \mathrm{c} \mathrm{Th}^{228}$ injected $/ \mathrm{kg}$, and 0.512 is the product of the riecessary conversion factors. The minimum estimate is .

$$
\begin{gathered}
\frac{\mathrm{dD}_{\min }}{\mathrm{dt}}=\frac{\mathrm{dD} \max }{\mathrm{dt}} \cdot-\left\{0.091 \mathrm{e}^{-0.00097 t}+0.025 \mathrm{e}^{-0.0003 t}\right\} x \\
13.3 \mathrm{e}^{-\lambda \mathrm{Th}^{228 t} \times \mathrm{I} \times 0.512}-0.62 \mathrm{e}^{-0.00197 t}-0.17 \mathrm{e}^{-0.0013 t} \mathrm{I} \mathrm{rads} / \mathrm{day}
\end{gathered}
$$

Integrating Eqs. (79) and (80) gives the maximum and minimum average radiation dose to bone:

$$
D_{\max }=9300\left(1-e^{-0.00114 t}\right) I \text { rads }
$$

and

$$
\mathrm{D}_{\min }=\mathrm{D}_{\max }-315\left(1-\mathrm{e}^{-0.00197 t}\right)-130\left(1-\mathrm{e}^{-0.0013 t}\right) \mathrm{I} \text { rads }(82)
$$

The actual dose rate is most certainly greater than the calculated minimum, Eq. (80), for the two assumptions, 1) that soft tissues retain all $\operatorname{Tn}^{220}$ produced there but bone does not, and 2) that no $\mathrm{Pb}^{212}$ leaves bone even though $\mathrm{Ra}^{224}$ is known to leave bone and $\operatorname{Tn}^{220}$ is assumed to do so, are quite extreme.

The maximum calculated dose rate is based on more realistic assumptions which are consistent with the short half-period of $\operatorname{Tn}^{220}$ and the correlation between the biological rate of decrease of $\mathrm{Pb}^{212}$ in blood cells and that for $\mathrm{Ra}^{224}$ in liver and spleen. Eqs. (79) and (81) appear to be the equations of choice. Also, the difference between the two is small, at 100 and 1000 days, $\mathrm{dD}_{\min } / \mathrm{dt}$ is 0.931 and $0.961 \mathrm{dD}_{\max } / \mathrm{dt}$, 
respectively. Thus, any overestimation of bone dose rate by Eq. (79) is small and not significant with respect to other uncertainties in these studies.

Although the $\operatorname{Tn}^{220}$ problem does not have a large effect on the calculation of average radiation dose rate to bone, we are completely lacking in information on its effect on local bone dose rates.

Another problem for which we lack information is the effect of the decay of $\mathrm{Tn}^{220}$ and $\mathrm{Po}^{216}$ in blood on the observed toxicity of $\mathrm{Th}^{228}$. Since we have only measured the sum of the effects of $\mathrm{Tn}^{220}$ and $\mathrm{Pb}^{212}$ migrations and cannot separate them, we can only set an upper limit for the energy dissipated. From Eq. (77) it is

Max. rate of energy release from $\operatorname{Tn}^{220}$ and $P^{216}$ decay in blood

$$
6200 \text { I W } e^{-0.00197 t} \text { engs/day }
$$

where $W$ is the dog weight at injection. This energy is in addition to that which results from decay of $\mathrm{Pb}^{212}$ plus decay products in or on blood cells. It is given by

Rate of energy release from $\mathrm{Pb}^{212}$ and decay products on or in blood cells =

$$
1950 \text { I W } \mathrm{e}^{-0.00197 t} \mathrm{ergs} / \mathrm{day}
$$

We lack specific information on the sites which receive the energy, Eqs. (83) and (84). Some possible consequences are damage to capillary walls 
with ensuing decrease in effectiveness of the vascular system, and damage of critical cells in tissues which fall within range.

Estimation of even average radiation dose rates to soft tissues is severely limited by the $\operatorname{Tn}^{220}$ problem. As an example, consider the liver. Using Eqs. (52), (51), and (53) and the average weight at injection of $9.04 \mathrm{~kg}$ for these 14 dogs, (the average liver weight given above is 335 g) we calculate the dose rate for the two cases of complete retention and complete loss of $\operatorname{Tn}^{220}$.

If $L\left(\operatorname{Tn}^{220}\right)=L\left(\mathrm{Ra}^{224}\right)$, then

$$
\frac{d D_{\max }}{d t}=2.32 I e^{-0.0020 t}
$$

and if

$$
\frac{\mathrm{dD}_{\min }}{\mathrm{dt}}=1.37 \mathrm{e}^{-0.0020 t}
$$

Here the minimum is only 0.59 the maximum. Also some part of the energy given in Eqs.' (83) and (84) results in some localized irradiation of the liver.

It is interesting to note the higher liver dose rate, Eq. (85), does not differ greatly during the first year from that for dogs given abuut $2.7 \mu \mathrm{c} \mathrm{Pu}{ }^{239} / \mathrm{kg}(5-$ level) ,. (12). For these dogs,

$$
\frac{\mathrm{dD}}{\mathrm{dt}}\left(\mathrm{Pu}^{239}\right)=2.46 \mathrm{I} \mathrm{e}^{-0.00116 t}
$$

For this group of 22 dogs, mean weight at injection $=9.58 \mathrm{~kg}$, and mean 
liver weight $=310 \mathrm{~g}$.

Since kidney damage has been observed in 4- and 5-level Th $^{228}$ dogs (1), and since the urinary excretion of both $\mathrm{Th}^{228}$ (2) and $\mathrm{Ra}^{224}$ (see above) decreases more rapidly in the case of 4-level dogs than for lower level dogs, it is appropriate to estimate some average radiation dose rates to the kidneys. Of the 13 dogs listed in Table VII, kidney measurements are complete for 8 of them. The maximum and minimum dose rates, assuming complete retention and complete loss of $\mathrm{Tn}^{220}$, respectively, can be calculated easily from Eqs. (57, 58, 59, and 60). However, the weight of kidneys of the 8 dogs ranged from $32.1 \mathrm{~g}$ to $79.1 \mathrm{~g}$, a factor of 2.5 . For this reason it seems more appropriate to tabulate individual values for the 8 dogs, and these appear in Table $X$. Since the activity of $\operatorname{Ra}^{224}$ is less than that of either $\mathrm{Th}^{228}$ or $B i^{212}$, the maximum and minimum calculations do not differ as much as do those"for the liver.

\section{TABLE $X$}

Average Radiation Dose Rates to Kidneys at Time of Death

\begin{tabular}{|c|c|c|c|c|c|}
\hline Dog & $\begin{array}{l}\text { Days } \\
\text { After } \\
\text { Injection }\end{array}$ & $\begin{array}{l}\text { Wt. of } \\
\text { Kidneys } \\
\text { (gs) }\end{array}$ & $\begin{array}{l}I= \\
\mu \mathrm{cTh} 228 / \mathrm{kg} \\
\text { Injected }\end{array}$ & $\begin{array}{l}\mathrm{dD}_{\max } / \mathrm{dt} \\
(\text { rads } / \text { day })\end{array}$ & $\begin{array}{l}\mathrm{dD} \cdot \min / \mathrm{dt} \\
(\mathrm{rads} / \mathrm{day})\end{array}$ \\
\hline $\mathrm{T} 5 \mathrm{~T} 5$ & 65 & 59.0 & 2.32 & $0.24 I \mathrm{~W}^{\mathrm{a}}=5.6$ & $0.21 \mathrm{IW}=4.9$ \\
\hline M4T2 & 78 & 79.1 & 0.090 & $0.19 I W=0.22$ & $0.14 \mathrm{IW}=0.16$ \\
\hline M2T5 & 97 & 61.0 & 2.63 & $0.18 I W=3.9$ & $0.14 \mathrm{IW}=3.0$ \\
\hline F3T3 & 547 & 61.5 & 0.272 & $0.16 \mathrm{IW}=0.52$ & $0.14 I W$ \\
\hline $\mathrm{MlT} 4$ & 645 & 32.5 & 0.882 & $0.069 \mathrm{IW}=0.51$ & $0.058 \mathrm{IW}=0.43$ \\
\hline M4T3 & 801 & 46.0 & 0.285 & $0.098 \mathrm{IW}=0.27$ & $0.086 I W=0.24$ \\
\hline M2T4 & 833 & 32.1 & 0.916 & $0.057 \mathrm{IW}=0.43$ & $0.043 \mathrm{TW}=0.32$ \\
\hline MIT3 & 988 & 75.8 & 0.301 & $0.060 \mathrm{IW}=0.16$ & $0.048 \mathrm{IW}=0.13$ \\
\hline
\end{tabular}

a $W=$ weight at injection 
Further, in connection with the dose level effect on urinary excretion of $\mathrm{Th}^{228}$ and $\mathrm{Ra}^{22.4}$, two factors should be noted, 1) there did not appear to be a dose level effect on urinary volume; 2) measurements of $\mathrm{Th}^{228}$ and $\mathrm{Ra}^{224}$ in plasma were too limited to permit examination for a dose level effect, so that we cannot differentiate between decreased kidney ability to excrete $\mathrm{Th}^{228}$ and $\mathrm{Ra}^{224}$ and decreases in the $\mathrm{Th}^{228}$ and $\mathrm{Ra}^{224}$ brought to the kidneys by the blood. Fecal excretion was depressed, too.

A final comment is that, while the elusiveness of $\mathrm{Tn}^{220}$ and the limited extent of the $\mathrm{Pb}^{212}$ and $\mathrm{Bi}^{212}$ measurements impose some limitations on the results, the most important limitation would seem to be that many of the measurements were made on higher level terminally ill dogs.

\section{References}

1. T. F. Dougherty, B. J. Stover, J. H. Dougherty, W. S. S. Jee, C. W. Mays, C. E. Rehfeld, W. R. Christensen, and H. C. Goldthorpe, Studies of the biological effects of Ra226; Pu239, Ra228, Th228 and $\mathrm{Sr}^{90}$ in adult beagles, Radiation Research 17, 625-681 (1962).

2. B. J. Stover, D. R. Atherton, N. Keller, and D. S. Buster, Metabolism of $\mathrm{Th}^{228}$ decay series in adult beagle dogs. I. Th228 (RdTh). Radiation Research 12, 657-671 (1960).

3. M. A. Van Dilla, B. J. Stover, and J. S. Arnold, On the retention and translocation of $\mathrm{Ra}_{224}(\mathrm{ThX})$ in dogs, Am. J. Roentgenol. Radium Therapy Nuclear Med. $\underline{77}$, 503-510 (1957).

4. C. W. Mays, R. Haldin, and M. A. Van Dilla, Thoron exhalation in radiothorium-burdened beagles. Radiation Research $\underline{9}, 438-444$ (1958)。

5. D. Strominger, J. H. Hollander, and G. T. Seaborg, Table of isotopes, Revs. Modern Phys. 30, 585-904 (1958).

6. M. A. Van Dilla, B. J. Stover, R. L. Floyd, D. R. Atherton, and D. H. Taysum, Radium ( $\mathrm{Ra}^{226}$ ) and radon $\left(\mathrm{Em}^{222}\right)$ metabolism in dogs, Radiation Research 8, 417-437 (1958). 
7. B. J. Stover, $\mathrm{Pb}^{212}$ (ThB) tracer studies in adult beagle dogs. Proc. Soc。Expt1. Biol。Med。100, 269-272 (1959).

8. C. R. Richmond, J. E. Furchner, P. N. Dean, and P. Mçililiams, Electronic processing and analysis of metabolic data, Health Physics 10, 3-13 (1964).

9. E。 B. Reeve, M。 I. Gregerson, T. H. Allen, and H. Sear, Distribution of cells and plasma in the normal and splenectomized dogs and its influence on blood volume estimates with $\mathrm{P}^{32}$ and $\mathrm{T}-1824, \mathrm{Am} . \mathrm{J}$. Physio1。175, 195-203 (1953).

10. J。E. Parkinson and J. H. Dougherty, Effect of internal emitters on red cel1 and plasma volumes of beagle dogs, Proc. Soc. Expt1. Biol. Med。 97, 722-725 (1958).

11. J. H. Dougherty and J. E. Parkinson, Private communication.

12. B. J. Stover, D. R. Atherton, F. W. Bruenger, and D. S. Buster, Further studies of the metabolism of $\mathrm{Pu}^{239}$ in adult beagles, Health Physics $\underline{8}, 589-597$ (1962)。 